\title{
Model Reduction of Flexible Multibody Systems
}

with application to large-stroke compliant precision mechanisms

\section{Steven E. Boer}




\section{MODEL REDUCTION OF FLEXIBLE MULTIBODY SYSTEMS}

WITH APPLICATION TO LARGE-STROKE COMPLIANT PRECISION MECHANISMS 


\section{Composition of the graduation committee:}

Chairman and secretary:

prof.dr. G.P.M.R. Dewulf

University of Twente

Promoter and assistent-promoters: prof.dr.ir. J.B. Jonker

University of Twente dr.ir. R.G.K.M. Aarts

University of Twente dr.ir. D.M. Brouwer

University of Twente

Members:

prof. O. Brüls

prof.dr.ir. A. de Boer

University of Liège

University of Twente

prof.dr.ir. H. van der Kooij

University of Twente / Delft University of

Technology

dr.ir. M.H.M Ellenbroek

University of Twente

The work described in this thesis was performed at the Mechanical Automation group of the Faculty of Engineering, University of Twente, P.O. Box 217, 7500 AE Enschede, The Netherlands.

This research is financially supported by the Dutch association Point-One, project MOV-ET PNE08006, by the Dutch Department of Economic Affairs, Agriculture and Innovation.

On the cover, the seventh base vector of the modal subspace as a function of a prescribed path of a two degree of freedom large-stroke compliant positioning mechanism. Cover design by Robin Boer.

Model Reduction of Flexible Multibody Systems

S.E. Boer

PhD Thesis, University of Twente, Enschede, The Netherlands

December 2013

ISBN 978-90-365-3574-8

DOI 10.3990/1.9789036535748

Copyright (C) 2013 by S.E. Boer, The Netherlands

Printed by Ipskamp Drukkers. 


\title{
MODEL REDUCTION OF FLEXIBLE MULTIBODY SYSTEMS
}

WITH APPLICATION TO LARGE-STROKE COMPLIANT PRECISION MECHANISMS

\author{
PROEFSCHRIFT
}

ter verkrijging van

de graad van doctor aan de Universiteit Twente,

op gezag van de rector magnificus,

prof. dr. H. Brinksma,

volgens besluit van het College voor Promoties

in het openbaar te verdedigen

op donderdag 5 december 2013 om 14.45 uur

door

Steven Ewoud Boer

geboren op 31 maart 1985

te Groningen 
Dit proefschrift is goedgekeurd door de promotor: prof.dr.ir. J.B. Jonker

en de assistent-promotoren:

dr.ir. R.G.K.M. Aarts

dr.ir. D.M. Brouwer 


\section{Samenvatting}

Het gebruik van numerieke simulaties is essentieel voor het bepalen van de prestaties en structurele integriteit van mechanismen en robots. Er worden steeds hogere eisen gesteld aan de specificaties van deze apparaten en dus ook aan de nauwkeurigheid van de numerieke simulaties. Door het gebruik van complexere modellen kan de simulatie nauwkeurigheid verhoogd worden. Echter, heeft dit tot gevolg dat de rekentijd van de simulaties langer wordt. Het toepassen van model reductie technieken op de onderliggende modellen kan een uitkomst bieden om de rekentijd te verkorten, terwijl de beoogde nauwkeurigheid behouden blijft. In dit proefschrift wordt onderzocht hoe model reductie technieken toegepast kunnen worden voor het reduceren van flexibele multibody modellen. Het startpunt is een numerieke aanpak die gebaseerd is op niet-lineaire eindige elementen, zoals het balk en scharnier element. Mechanismen die bestaan uit balk-achtige onderdelen kunnen met deze methode goed gesimuleerd worden. Echter, als de onderdelen niet balk-achtig zijn, of als het mechanisme uit erg veel onderdelen bestaat, kan het multibody model dusdanig complex worden waardoor de benodigde rekentijd onacceptabel wordt. Daarom zijn er twee verschillende types van model reductie onderzocht. Het eerste type, genaamd component model reductie, kan toegepast worden om op een efficiënte en nauwkeurige manier complex gevormde flexibele onderdelen te modelleren. Het tweede type, genaamd systeem model reductie, probeert vervolgens de complexiteit van het hele niet-lineaire multibody model te reduceren. Beide types van model reductie worden gedemonstreerd aan de hand van het modelleren van een grote-slag elastisch precisie mechanisme met twee vrijheidsgraden.

Voor het eerste type is een zogenaamde 'two-node non-linear superelement' ontwikkeld zodat onderdelen van arbitraire vorm met twee interface oppervlakken naar andere onderdelen, efficiënt opgenomen kunnen worden in een multibody model. Er wordt aangenomen dat de vervormingen van het superelement klein blijven ten opzichte van een meedraaiend assenstelsel. Met deze aanname kunnen de dynamische eigenschappen van het superelement bepaald worden met behulp van een gereduceerd lineair eindig elementen model. De belangrijkste bijdrage van de voorgestelde methode is het gebruik van 'deformable-interface modes' die de 
vervorming van de interface oppervlakken van het superelement beschrijven. Dit maakt het mogelijk om superelementen elastisch met elkaar te verbinden. Hiermee kan een onderdeel ook gemodelleerd worden met een combinatie van meerdere superelementen. Grote vervormingen van het onderdeel kunnen op deze manier nauwkeurig beschreven worden, zolang de vervormingen van de individuele superelementen klein blijven ten opzichte van hun meedraaiende assenstelsels. Absolute coördinaten worden gebruikt om de posities en oriëntaties van de interface oppervlakken van een superelement te beschrijven. Hierdoor kunnen de superelementen eenvoudig met elkaar verbonden worden om een nauwkeurig model te creeëren van een compleet mechanisme. Het grote-slag elastisch precisie mechanisme is op deze manier gemodelleerd. Dit model bleek nauwkeurig en een stuk minder complex te zijn ten opzichte van een model verkregen met klassieke eindig elementen. Echter, waren de rekentijden van de simulaties nog te lang om bijvoorbeeld snel gesloten lus simulaties uit te kunnen voeren.

Daarom is een systeem model reductie methode ontwikkeld, zodat elastische mechanismen die een grote slag ondergaan toch efficiënt gesimuleerd kunnen worden. Voor een nauwkeurig model van dit soort mechanismen is het belangrijk dat geometrische niet-lineariteiten goed meegenomen worden, aangezien de stijfheidseigenschappen aanzienlijk kunnen veranderen bij grote vervormingen. Om grote vervormingen nauwkeurig te kunnen modelleren zijn er veel eindige elementen nodig, zodat de vervormingen van de elementen zelf klein kunnen blijven. Het gebruik van meer elementen verhoogt, behalve het aantal vrijheidsgraden, ook de hoogste eigenfrequentie van het model. Deze verkleint op zijn beurt de toelaatbare grote van de tijdstappen van expliciete tijdsintegratoren. Om grotere tijdstappen en daarmee snellere simulaties mogelijk te kunnen maken, probeert de voorgestelde systeem model reductie methode ongewenste eigenmodes met hoge eigenfrequenties te onderdrukken. Dit terwijl de geometrische niet-lineariteiten en gewenste eigenmodes behouden blijven in het gereduceerde model. De onderdrukking van de ongewenste eigenmodes wordt gerealiseerd door relaties aan te brengen tussen de vrijheidsgraden van het model. Deze relaties kunnen zowel lineair als niet-lineaire zijn. De nietlineaire variant is gebruikt om het model van het grote-slag elastisch precisie mechanisme te reduceren. Het is aangetoond dat deze methode correct de gewenste eigenmodes behoudt, terwijl de benodigde rekentijd van de simulaties significant verminderd. 


\section{Summary}

Numerical simulations are essential to determine the characteristics, performance and structural integrity of mechanisms and robots. With increasingly higher demands on the specifications of such devices, the demands on the accuracy of the numerical models increases as well. Increasing the complexity of the models, inherently increases the computational time of the simulations. Model reduction techniques can offer a reduction of the simulation time, while maintaining sufficient accuracy. In this work, the modelling of flexible multibody systems is considered. The starting point is a numerical approach based on non-linear finite elements such as beams, trusses and hinges. In particular the spatial beam element has proven to offer a numerical efficient analysis of mechanisms that consist of beam-like components. However, for systems with complex-shaped parts, or for systems composed of a rather large number of beams, the dimensionality of the model increases. Hence, two types of model reduction techniques are investigated for the efficient and accurate modelling of such flexible multibody systems. The first type deals with the efficient and accurate modelling of individual components that may be flexible and have a complex geometry. This is referred to as component model reduction. The second type attempts to reduce a complete non-linear multibody system and can therefore be referred to as a system model reduction approach. Both methods are demonstrated by modelling a largestroke two degree of freedom compliant positioning mechanism.

For the first type, a non-linear two-node superelement is proposed for efficient modelling of arbitrary-shaped flexible members with two interfaces in a flexible multibody model. The formulation is based on a small rotation and displacement hypothesis in a local co-rotational frame. Component mode substructuring methods can then be used to determine the dynamical properties of the superelement from a linear finite element model. The key contribution is the inclusion of so-called deformable-interface modes to model the deformability of the interface surfaces. This allows for an elastic connection to other superelements. With this capability, a component can be modelled with a number of superelements and its dynamical properties can be accurately analysed even for large deflections provided that the deformations remain small with respect to the co-rotational frames. Unlike more 
conventional approaches that define the reduced model in terms of absolute and relative coordinates, the presented superelement approach uses only absolute coordinates. This allows for an easy assembly of superelements to create an accurate model of a mechanism or robot. For the compliant two degree of freedom mechanism, the obtained model is accurate and substantially reduced in size compared to a classical finite element model. However, the model is still too complex to obtain fast simulation times to perform for example closed-loop time response simulations.

Therefore, a system model reduction method is proposed for efficient timeintegration of compliant mechanism models that undergo large deflections. Of particular importance for the modelling of this class of mechanisms is the accurate description of geometric non-linearities, as stiffness characteristics can change significantly during deflection. The modelling of large deflections requires a sufficient number of finite elements to ensure that deformations remain small in a co-rotational context. Increasing the number of elements, increases, besides the number of degrees of freedom, also the largest eigenfrequency in the model. This reduces the allowable step size, in particular for explicit time-integrator methods. The proposed reduction method aims to suppress the high frequency vibrational modes which are not important for the desired simulation results, while retaining the geometric non-linearities in the reduced model. For this purpose constraint relations that inhibit the motion of unwanted higher vibrational modes are added between the independent generalized coordinates that define the configuration of the model. These constraint relations can be linear or non-linear. The non-linear variant, which is referred to as the interpolated basis method, is used to reduce the model of the two degree of freedom compliant mechanism. It is demonstrated that the method yields a reduced model that correctly preserves the vibrational modes of interest, while yielding a significant increase in the computational efficiency for the time-integration of the equations of motion. 


\section{Nomenclature}

\section{Notation}

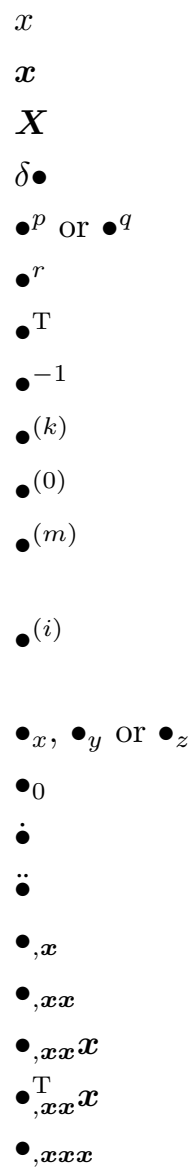

A scalar $x$

A vector $\boldsymbol{x}$

A matrix $\boldsymbol{X}$

Virtual variations of $\bullet$ or pertubations of $\bullet$

Scalar, vector or matrix quantity at the $p$ or $q$ side of a finite element

Average Euler parameters or rotation matrix of the $p$ and $q$ side

Transpose of •

Inverse of $\bullet$

Quantity of the $k^{\text {th }}$ element

Subset of coordinates of $\bullet$ that are prescribed to be fixed

Subset of coordinates of $\bullet$ that are associated with the degrees of freedom of the model

Quantity associated with the $i^{\text {th }}$ node of the linear FE model, or a collection of matrices

The $x, y$ or $z$ component of $\bullet$

Initial state or value of $\bullet$

First time derivative of $\bullet$

Second time derivative of $\bullet$

Derivative of $\bullet$ to $\boldsymbol{x}$ (Jacobian matrx)

Second derivative of $\bullet$ to $\boldsymbol{x}$ (Hessian matrix)

Denotes multiplication over the last dimension of $\bullet_{, \boldsymbol{x} x}$, i.e. $\bullet_{i, j k} x_{k}$

Denotes multiplication over the first dimension of $\bullet_{, \boldsymbol{x} \boldsymbol{x}}$, i.e. $\bullet_{i, j k} x_{i}$

Third derivative of $\bullet$ to $\boldsymbol{x}$ 
Kinematic quantity related to the reference frame of the linear superelement

\section{Abbreviations}

$\begin{array}{ll}\text { AMI } & \text { Adaptive modal integration } \\ \text { DAE } & \text { Differential algebraic equation } \\ \text { FBM } & \text { Fixed basis method } \\ \text { FE } & \text { Finite element } \\ \text { GMP } & \text { Global modal parametrization } \\ \text { IBM } & \text { Interpolated basis method } \\ \text { MSI } & \text { Modal subspace interpolation } \\ \text { ODE } & \text { Ordinary differential equation } \\ \text { SVD } & \text { Singular value decomposition }\end{array}$

\section{Vector functions}

$\mathcal{D}$

$\mathcal{E}$

$\mathcal{F}$

$\mathcal{G}$

$\mathcal{P}$

$\mathcal{Q}$

$\mathcal{V}$
Deformation vector function

Geometric transfer function, related to deformation coordinates

Geometric transfer function, related to nodal coordinates

Constraint vector function

Path coordinates vector function

The gross reference motion

The interpolated modal subspace

\section{Vectors Latin}

e

$e_{l}$

$f$

$h$

$n$

$q$

$\boldsymbol{r}$

$\boldsymbol{s}, s$

$\bar{u}$

Unit vector of a coordinate system

Unit vector describing the direction of the element axis

Force vector

Velocity dependent inertia forces

Unit vector of the inertial coordinate system, or unit normal

Vector of independent generalized coordinates

Cartesian position vector

Vector of configuration coordinates, or the configuration coordinate

Nodal displacements of a linear FE model 


$\begin{array}{ll}\boldsymbol{v} & \text { Velocity vector } \\ \boldsymbol{v}_{i} & \text { The } i^{\text {th }} \text { eigenvector } \\ \boldsymbol{x} & \text { Vector of nodal coordinates } \\ \boldsymbol{z} & \text { Vector of reduced coordinates }\end{array}$

\section{Vectors Greek}

$\varepsilon$

$\eta$

$\bar{\eta}$

$\overline{\boldsymbol{\eta}}_{d}$

$\overline{\boldsymbol{\eta}}_{f}$

$\lambda$

$\bar{\mu}$

$\boldsymbol{\sigma}$

$\bar{\varphi}$

$\omega$
Vector of deformation coordinates

Vector of modal coordinates

Generalized coordinates of the linear superelement

Coordinates associated with deformable-interface modes

Coordinates associated with fixed-interface normal modes

Euler parameters

Boundary coordinates of the linear superelement

Vector of stress resultants

Rotation vector of the linear superelement

Angular velocity vector

\section{Matrices Latin}

0 The zero matrix

$\boldsymbol{A}$ Diagonal matrix relating deformation coordinates

$\boldsymbol{B} \quad$ Superelement velocity transformation matrix

$\boldsymbol{B}_{1}, \boldsymbol{B}_{2}$ or $\boldsymbol{B}_{3} \quad$ Three different superelement velocity transformation matrices

$\boldsymbol{C}_{\boldsymbol{q}} \quad$ Velocity sensitivity matrix in terms of independent generalized coordinates

$\boldsymbol{C}_{\boldsymbol{z}} \quad$ Velocity sensitivity matrix in terms of reduced coordinates

D Element damping matrix

$\boldsymbol{D}_{\boldsymbol{q}} \quad$ Damping matrix in terms of independent generalized coordinates

$\boldsymbol{D}_{\boldsymbol{z}} \quad$ Damping matrix in terms of reduced coordinates

$\boldsymbol{G}_{\boldsymbol{q}} \quad$ Geometric stiffness matrix in terms of independent generalized coordinates

$\boldsymbol{G}_{\boldsymbol{z}} \quad$ Geometric stiffness matrix in terms of reduced coordinates

I The identity matrix

$\boldsymbol{K}_{\boldsymbol{q}} \quad$ Structural stiffness matrix in terms of independent generalized coordinates 
$\boldsymbol{K}_{\boldsymbol{z}} \quad$ Structural stiffness matrix in terms of reduced coordinates

$\overline{\boldsymbol{K}}_{\mathrm{FEM}} \quad$ Finite element model stiffness matrix

$M \quad$ Element mass matrix

$\boldsymbol{M}_{\boldsymbol{x}} \quad$ Complete assembled mass matrix

$\boldsymbol{M}_{\boldsymbol{q}} \quad$ Mass matrix in terms of independent generalized coordinates

$\boldsymbol{M}_{\boldsymbol{z}} \quad$ Mass matrix in terms of reduced coordinates

$\bar{M} \quad$ Linear superelement mass matrix

$\overline{\boldsymbol{M}}_{\mathrm{FEM}} \quad$ Finite element model mass matrix

$\boldsymbol{N}_{\boldsymbol{q}} \quad$ Dynamic stiffness matrix in terms of independent generalized coordinates

$\boldsymbol{N}_{\boldsymbol{z}} \quad$ Dynamic stiffness matrix in terms of reduced coordinates

$\boldsymbol{R} \quad$ A rotation matrix

$\boldsymbol{S} \quad$ Element stiffness matrix

$\overline{\boldsymbol{S}} \quad$ Linear superelement stiffness matrix

$\boldsymbol{V} \quad$ Matrix containing the constraint modes, projection matrix or a matrix containing eigenvectors

$\boldsymbol{V}_{e} \quad$ Matrix containing a subset of constraint modes spanning the subspace associated with purely elastic deformations

$\boldsymbol{W} \quad$ Matrix containing fixed-interface and deformable-interface modes, or a dummy matrix to compute the FBM and IBM

$\boldsymbol{W}_{d} \quad$ Matrix containing deformable-interface (warping) modes

$\boldsymbol{W}_{f} \quad$ Matrix containing fixed-interface normal modes

\section{Matrices Greek}
$\Lambda, \bar{\Lambda}$
A $3 \times 4$ matrix with Euler parameters 


\section{Contents}

Samenvatting v v

Summary vii

Nomenclature $\quad$ ix

Contents $\quad$ xiii

1 Introduction $\quad \mathbf{1}$

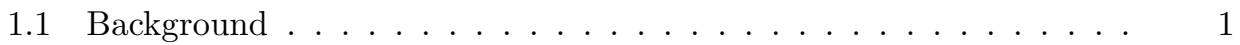

1.2 Research objectives . . . . . . . . . . . . . . . . . . . . . . 4

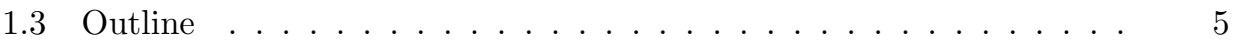

$\begin{array}{lll}\text { I Component model reduction: superelements } & 7\end{array}$

2 A non-linear two-node superelement for use in flexible multibody $\begin{array}{ll}\text { systems } & 9\end{array}$

2.1 Introduction . . . . . . . . . . . . . . . . . . . . . . . . . 9

2.2 Linear two-node superelement . . . . . . . . . . . . . . . . . . 12

2.2.1 Boundary coordinates and constraint modes . . . . . . . . . 12

2.2.2 Potential energy . . . . . . . . . . . . . . . . . . . 13

2.2 .3 Kinetic energy . . . . . . . . . . . . . . . . . . 14

2.3 Non-linear two-node superelement . . . . . . . . . . . . . . . . . 15

2.3.1 Nodal coordinates and deformation modes . . . . . . . . 15

2.3.2 Potential energy . . . . . . . . . . . . . . . . . 18

2.3.3 Kinetic energy . . . . . . . . . . . . . . . . . . 19

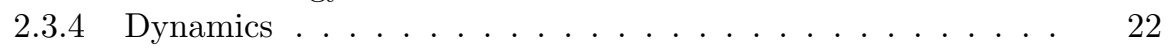

2.4 Numerical validation . . . . . . . . . . . . . . . . . . . . . . . . . . . . . . . . . . . . . . . .

2.4.1 Spinning beam .................... 24

xiii 
2.4.2 Spinning beam with out-of-plane bending . . . . . . . . . . . 25

2.4.3 Unbalanced rotating shaft . . . . . . . . . . . . . . . . 28

2.5 Conclusions . . . . . . . . . . . . . . . . . . 31

3 A non-linear two-node superelement with deformable-interface surfaces for use in flexible multibody systems

3.1 Introduction . . . . . . . . . . . . . . . . . . . . . . 33

3.2 Linear two-node superelement . . . . . . . . . . . . . . . . . . . . . . . . . . . . . . 36

3.2.1 Coordinates and components modes . . . . . . . . . . . 36

3.2 .2 Potential energy . . . . . . . . . . . . . . . . . . . . . . . . 40

3.2 .3 Kinetic energy . . . . . . . . . . . . . . . . . . . . 41

3.3 Non-linear two-node superelement . . . . . . . . . . . . . . . 41

3.3.1 Coordinates and modes ................ . . . . . . . 41

3.3.2 Potential energy . . . . . . . . . . . . . . . . . . . . . 44

3.3.3 Kinetic energy . . . . . . . . . . . . . . . . . . . . . 45

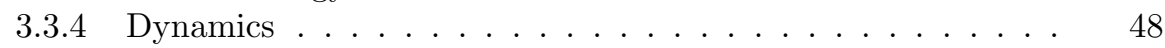

3.4 Superelements with deformable-interface modes . . . . . . . . . . 49

3.4.1 Assembly of superelements . . . . . . . . . . . . . . 49

3.4.2 Determining compatible warping modes . . . . . . . . . . 50

3.5 Numerical examples . . . . . . . . . . . . . . . . . . 51

3.5.1 Large deflections of a compliant flexure . . . . . . . . . . 52

3.5.2 Modelling of a complex-shaped flexible component exhibiting small deflections . . . . . . . . . . . . . . 55

3.5.3 Time-response of a complex-shaped flexible component attached to a compliant cross-hinge . . . . . . . . . . . . 57

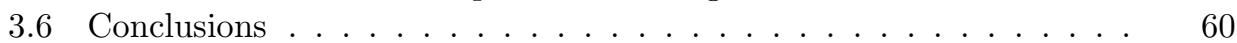

4 Modelling a large-stroke compliant mechanism with superelements 63

4.1 Large-stroke compliant mechanism . . . . . . . . . . . . . 63

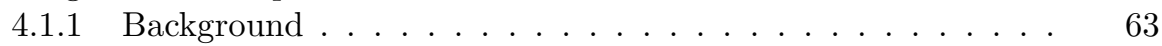

4.1.2 Mechanism details . . . . . . . . . . . . . . . . . 65

4.1.3 Exact constraint design . . . . . . . . . . . . . . . 66

4.2 Spacar mechanism model . . . . . . . . . . . . . . . . . . . . 67

4.2.1 Complex-shaped flexible members . . . . . . . . . . . 69

4.2 .2 Cross-hinge assemblies . . . . . . . . . . . . . . . . . 70

4.3 Ansys mechanism model . . . . . . . . . . . . . . . . . 73

4.4 Comparison of eigenfrequencies . . . . . . . . . . . . . . . . . 74

4.5 Conclusions ........................... 78

5 Model reduction for efficient time-integration of planar flexible multibody models 
5.1 Introduction . . . . . . . . . . . . . . . . . . . . . 82

5.2 Finite element modelling . . . . . . . . . . . . . . . . . 83

5.2.1 Finite element representation . . . . . . . . . . . . 83

5.2 .2 Equations of motion . . . . . . . . . . . . . . 85

5.2.3 Linearized equations of motion in a reference configuration . $\quad 86$

5.3 Reduction of degrees of freedom . . . . . . . . . . . . . . . . 87

5.3.1 Motivation and approach .............. 87

5.3 .2 Modal basis . . . . . . . . . . . . . . . . . . . 88

5.3.3 SVD-modal basis . . . . . . . . . . . . . . . . . . 89

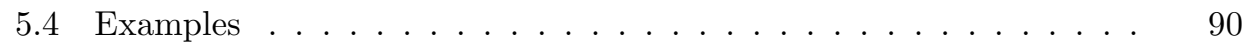

5.4.1 Compliant straight guidance . . . . . . . . . . . . 90

5.4 .2 Flexible manipulator . . . . . . . . . . . . . . . 94

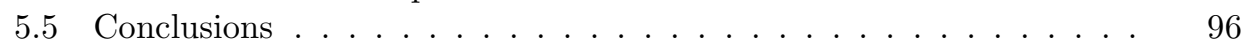

6 Model reduction for efficient time-integration of spatial flexible $\begin{array}{ll}\text { multibody models } & 97\end{array}$

6.1 Introduction . . . . . . . . . . . . . . . . . . . . . . . . 97

6.2 Finite element modelling . . . . . . . . . . . . . . . . . . . 100

6.2.1 Finite element representation . . . . . . . . . . . . . . 100

6.2.2 Equations of motion . . . . . . . . . . . . . . . . . 102

6.3 Reduction of degrees of freedom . . . . . . . . . . . . . . . . . . . . . . . . . . . . . . 103

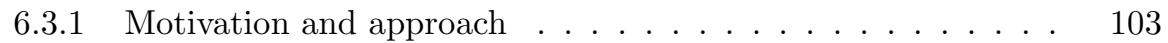

6.3.2 Fixed basis method (FBM) . . . . . . . . . . . . . . 105

6.3.3 Interpolated basis method (IBM) . . . . . . . . . . . . 108

6.4 Application example . . . . . . . . . . . . . . . . . 111

6.4.1 Compliant straight guidance model . . . . . . . . . . . . . 111

6.4.2 Reduction with fixed basis method . . . . . . . . . . . 113

6.4.3 Reduction with interpolated basis method . . . . . . . . . . 117

6.4.4 Comparison of the fixed and interpolated basis method . . . 120

6.5 Conclusions ...................... 120

7 Analysis and simulation of a large-stroke compliant mechanism 123

7.1 Model reduction along a predetermined path . . . . . . . . . . . 123

7.1.1 Applying the IBM for mechanisms with multiple degrees of

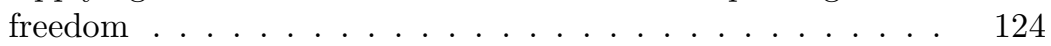

7.1 .2 Constrained eigenvectors . . . . . . . . . . . . . . . . . . . . . . 125

7.1.3 Unconstrained eigenvectors . . . . . . . . . . . . . 126

7.2 Model reduction of the large-stroke compliant mechanism . . . . . . 127

7.2.1 Reduced models . . . . . . . . . . . . . . . 127

7.2.2 Comparison of eigenfrequencies . . . . . . . . . . . . . . 129

7.2.3 Closed-loop time-response simulation . . . . . . . . . . . . 130

7.3 Conclusions .......................... 135

8 Conclusions and recommendations $\quad 137$

8.1 Conclusions . . . . . . . . . . . . . . . . 137 
8.1.1 Component model reduction . . . . . . . . . . . . . . 137

8.1.2 System model reduction . . . . . . . . . . . . . . . . 138

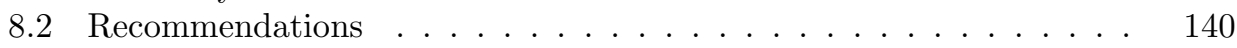

$\begin{array}{lr}\text { Appendices } & 143\end{array}$

A Transformation of velocities $\quad 145$

$\begin{array}{ll}\text { B Expressions for } \dot{B} & 147\end{array}$

$\begin{array}{lr}C \text { Expressions for } B^{*} & 149\end{array}$

D Equations of motion of a multibody system containing superelements $\quad 153$

E Linearized equations of motion $\quad 155$

$\begin{array}{lll}\text { F } & \text { Reduced linearized equations of motion } & 157\end{array}$

Dankwoord $\quad 159$

$\begin{array}{lr}\text { List of publications } & 161\end{array}$

$\begin{array}{ll}\text { References } & 165\end{array}$ 
Model reduction for flexible multibody systems is the overall topic of this thesis. In this chapter, the essence of model reduction is explained. Furthermore, a brief introduction is given on flexible multibody systems and on the application of model reduction methods for such systems. The research objectives of this thesis are discussed next, followed by the thesis outline.

\section{$1.1 \quad$ Background}

Numerical simulations are essential in the design phases of mechanisms and robots in order to determine their characteristics, performance and structural integrity before the actual fabrication of a costly prototype. To decrease the computational time of simulations, more powerful computers can be used. Following Moore's law [53], the number of transistors in integrated circuits, and with it the computational power of computers, has roughly doubled every two years over the last five decades. However, this trend does not result in a dramatic reduction of the computational time of simulations as there is also a tendency to use this increase in computational power for the development of more complex models that can provide results of increased accuracy. This again creates a demand for more computational power in order to reduce the computational time of these models. Figure 1.1 graphically depicts this vicious cycle. Though, for any given hardware setup and model complexity, shorter simulation times can be obtained by the application of model reduction tecniques.

The essence of model reduction is to only preserve the physical phenomena and quantities of interest from a model of high complexity, such that a much simpler model can be obtained which can (hopefully) be solved in a more efficient manner. The challenge of model reduction is how to obtain a reduced model that preserves the essential features from a model of higher complexity. There are many different ways of reducing the complexity of a model. Depending on the type of problem and the field of research, different model reduction methods are available. Most 


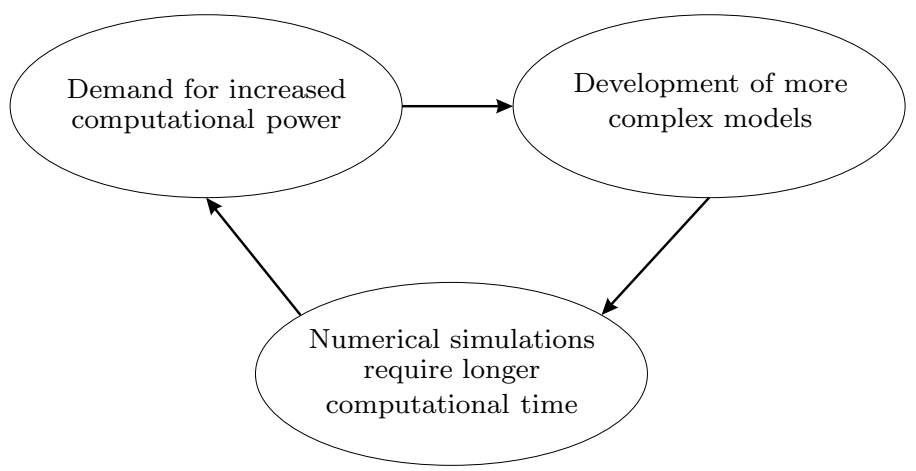

Figure 1.1: Increasing the computational power of computers, results in the development of more complex models, which in turn increases the computational time of the numerical simulations and again creates a demand for more powerful computers.

of the established methods are applied to linear time-invariant systems. Modal truncation is probably the best-known reduction method for this type of system. Here, the model is first transformed to an equivalent modal description and subsequently the contributions of vibrational modes with high resonance frequencies are disregarded. In structural engineering, other well-known methods are the Guyan-Irons reduction method [31], which preserves the static behaviour of a structure, and the Craig-Bampton substructuring method [23], which combines aspects of both modal truncation and the Guyan-Irons reduction method. In control engineering, reduction methods that preserve the time-response, the input-output behaviour or the dominant characteristics of transfer functions are often used. Examples are proper orthogonal decomposition [44], balanced truncation [52], and the application of the Lanczos [47] and Arnoldi [6] algorithms to construct Krylov subspaces to reduce the model.

Only a few of the reduction methods presented in literature are mentioned above. There are many more reduction methods each with their own advantages and disadvantages. Though, common to most of these methods is the assumption that the underlying model considered for reduction, is linear.

The equations that describe the dynamics of mechanisms and robots are highly non-linear. This is because the overall geometry of mechanisms can change significantly during operation due to the large rotations and translations of components. This thesis focuses on a class of mechanisms known as compliant mechanisms. To create mobility in such mechanisms, the elastic deflections of typically wireand sheet-flexures are utilized. This research is particularly concerned with the modelling of large-stroke compliant mechanisms because of their potential in high precision engineering. Such mechanisms do not suffer from friction or backlash, and have a low hysteresis, allowing high-precision and repeatability. For large-stroke applications, the deflection of the sheet-flexures can be significant (up to \pm 20 degrees). Two examples of large-stroke compliant mechanisms are shown in Fig. 1.2: 


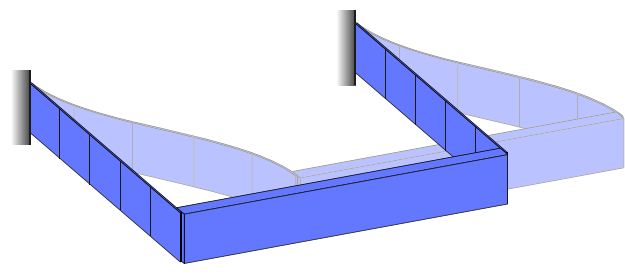

(a) Compliant straight-guidance

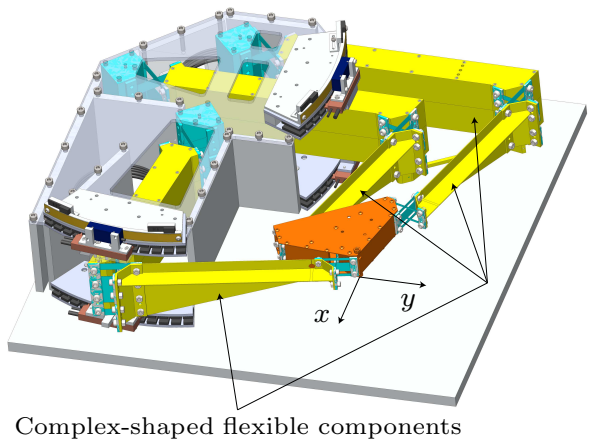

(b) Two degree of freedom large-stroke positioning mechanism

Figure 1.2: Examples of compliant mechanisms.

a compliant straight-guidance, and a two degree of freedom large-stroke positioning mechanism. Here, the mention of 'degree of freedom' in relation with a mechanism, refers to the intended mobility of that mechanism.

Flexible multibody modelling approaches are well-suited to describe these mechanisms. In a flexible multibody system, a mechanism is described by a collection of bodies that may or may not be rigid. They can be interconnected by joints, actuators, springs or dampers. Each body can make arbitrary large rigid body motions corresponding to the constraints imposed by the mechanical joints. Depending on the formulation, the bodies may exhibit small or large elastic deformations. In the case a body is only exhibiting small elastic deformations, linear stiffness models, such as those obtained from a classical finite element (FE) analysis, may be used to describe the deformations and to compute the stresses. With the FE method, accurate models of components of arbitrary shape can be obtained. The previously mentioned linear reduction methods may be employed to obtain a reduced description of the component. This is referred to as component model reduction.

The challenge of component model reduction is to include the reduced linear model in the non-linear environment of the flexible multibody system. The most intuitive method to achieve this is the floating frame of reference approach [62]. Here, attached to, or floating near each body is a reference frame whose position and orientation describes the mean rigid body motion in a fixed coordinate system. Absolute coordinates define the position and orientation of the reference frame, whereas relative coordinates are used to describe the small elastic deformations of the body with respect to the reference frame. The objective of component model reduction is then to reduce the number of relative coordinates that are necessary to describe the elastic deformations. A drawback of the floating frame of reference method is that additional non-linear algebraic constraint equations are required to relate the absolute and relative coordinates of interconnecting bodies. Furthermore, the formulation leads to a highly non-linear inertia matrix that exhibits a strong 
coupling between the reference motion and the deformation [61]. This non-linear inertia matrix can not be obtained completely from the linear reduced model. Instead, it has to be obtained by finite elements embedded in the multibody program for the modelling of a complex-shaped component.

A potential alternative for the inclusion of reduced linear models in a multibody system, is the generalized strain approach introduced by Besseling [9] and further developed by Jonker and Meijaard [38, 43, 50]. In this approach, a multibody system is modelled as an assembly of non-linear finite elements such as beams, trusses and hinges. For each element a fixed number of deformations modes are defined with respect to a co-rotational frame attached to the element. The deformation modes are characterized by deformation coordinates, so-called generalized strains, which are expressed as analytical functions of the nodal coordinates defined in a global inertial reference system. These functions allow for a fully non-linear representation of the rigid body motion and the use of absolute nodal coordinates allows for a straightforward interconnection of the elements. Furthermore, large deflection problems can be solved accurately provided that the element size is chosen sufficiently small such that the element deformations remain small as well. As a consequence, the application of linear stiffness models for the computation of the stresses is valid and this offers an opportunity for the inclusion of component reduced linear models.

Further model reduction can be obtained with the aid of system model reduction techniques. The aim of system model reduction is to obtain a non-linear reduced description of the entire flexible multibody model. Global modal parametrization (GMP) [18] and adaptive modal integration (AMI) [1] are examples of system model reduction methods applied to flexible multibody models. In these methods, the motion of the mechanism is separated in a non-linear nominal rigid body motion and a configuration dependent elastic deformation part. The elastic deformations are described by low frequency vibrational modes and are assumed to remain small with respect to the rigid body motion. This makes these methods less suited for the reduction of compliant mechanism models that undergo large deflections and therefore have no rigid body motion, such as those shown in Fig. 1.2. Nevertheless, the concept of considering only the low frequency vibrational modes also appears promising for the reduction of compliant mechanisms, provided that the large-stroke compliant motion can be described and the configuration dependency of the vibrational modes can be accounted for.

\section{$1.2 \quad$ Research objectives}

The main objectives of this thesis can be formulated as follows:

1. The implementation of a component model reduction method for multibody system analysis of compliant mechanisms using the generalized strain approach.

2. The development of system model reduction methods applied to flexible multibody systems for fast and accurate simulations of compliant mechanisms. 
The two degree of freedom large-stroke compliant positioning mechanism shown in Fig. 1.2(b), is used as an ultimate test case for the developed methods. Therefore, for the first objective, the efficient modelling of the complex-shaped flexible components shown in Fig. 1.2(b) and the correct modelling of the sheet flexures, are of particular concern. These components can be characterized by two interface surfaces, resulting in the development of a so-called non-linear two-node superelement.

With the superelement description of the first objective, an accurate model of the compliant mechanism of Fig. 1.2(b) can be developed. However, due to the large number of elements required to model such a mechanism, the computational time of numerical simulations is unacceptable. Therefore, a suitable system model reduction method needs to be developed and applied.

\subsection{Outline}

The thesis consists of two parts. The first part, consisting of Chapters 2, 3 and 4, deals with component model reduction. Chapters 5, 6 and 7 make up the second part, where the system model reduction methods are presented and applied.

Chapters 2, 3, 5 and 6 are written as journal paper contributions. This results in some overlap between Chapters 2 and 3, and between Chapters 5 and 6 . However, this has the advantage that each of these chapters can be read independently from the other chapters.

In Chapter 2, the implementation of the two-node non-linear superelement is investigated. It is assumed that the two interface surfaces which are used to connect the superelement to neighbouring elements, remain rigid.

In Chapter 3 the superelement method is extended with deformable-interface modes to model local deformations of the interface surfaces. This allows for a compliant connection to other superelements. It is discussed how a proper compliant interconnection of superelements should be obtained. In particular, the computation of modes that model the warping of the cross-section due to a twist of the component, are discussed.

Chapter 4 discusses the design of the two degree of freedom large-stroke compliant positioning mechanism of Fig. 1.2(b). With the aid of superelements, a complete multibody model of the mechanism is developed. A comparison is made between eigenfrequency results obtained with the developed model and those of a classical non-linear FE analysis.

In Chapter 5 a system model reduction method based on constraining the degrees of freedom of a planar flexible multibody model, is developed. Linear constraints, determined with a singular value decomposition (SVD) analysis, are applied to the degrees of freedom in such a way that high frequency vibrational modes are suppressed.

In Chapter 6, the method of Chapter 5 is extended to handle spatial mechanisms. The application of both linear and non-linear constraints are considered, yielding a fixed basis method and an interpolated basis method, respectively. 
In Chapter 7, the interpolated basis method of Chapter 6 is further developed so that it can be applied to reduce the model obtained in Chapter 4 of the compliant positioning mechanism shown in Fig. 1.2(b). With the reduced model, eigenfrequency results are computed along a prescribed path and compared to those of the unreduced model of Chapter 4. To conclude, a closed-loop time-response simulation of the reduced model is performed.

Conclusions are presented in Chapter 8 , followed by some recommendations for further research. 
Part I

Component model reduction: superelements 



\title{
A non-linear two-node superelement for use in flexible multibody systems
}

\begin{abstract}
A non-linear two-node superelement is proposed for the modelling of flexible complex-shaped members for use in multibody simulations. Assuming that the elastic deformations with respect to a co-rotational reference frame remain small, substructuring methods may be used to obtain reduced mass and stiffness matrices from a linear finite element model. These matrices are used in the derivation of potential and kinetic energy expressions of the non-linear two-node superelement. By evaluating Lagrange's equations, expressions for the internal and external forces acting on the superelement can be obtained. The inertia forces of the superelement are derived in terms of absolute nodal velocities and accelerations, which greatly simplifies the dynamic formulation. Three examples are included. The first two examples are used to validate the method by comparing the results with those obtained from non-linear beam element solutions. We consider a benchmark simulation of the spin-up motion of a flexible beam with uniform cross-section and a similar simulation in which the beam is simultaneously excited in the out-of-plane direction. Results from both examples show good agreement with simulation results obtained using non-linear finite beam elements. In a third example, the method is applied to an unbalanced rotating shaft, illustrating the potential of the proposed methodology for a more complex geometry.
\end{abstract}

\section{$2.1 \quad$ Introduction}

In flexible multibody systems, the non-linear flexible beam element is a popular tool for modelling flexible members with uniform cross-section because of accurate proven results for small and large deflections and because of its numerical efficiency. Due to these advantages they are included in many multibody modelling approaches. A variety of well established non-linear beam element formalisms can be found in literature $[28,62]$. In general, the derivations of the mass and stiffness

Published in: Multibody System Dynamics (S.E. Boer, R.G.K.M. Aarts, J.P. Meijaard, D.M. Brouwer, J.B. Jonker) 
matrices of a beam element are based on the elastic line concept, where the elastic line is described using polynomial expressions. This allows accurate modelling of slender components with a uniform cross-section. However, it is less suited for the modelling of complex-shaped flexible members where the cross-sectional properties are varying significantly over the length of the component. Nevertheless, the kinematic description of a non-linear beam element presented in [38] offers an opportunity to develop an efficient modelling approach of such components by combining this description with substructuring methods for the determination of the mass and stiffness properties, instead of using the elastic line concept.

The development of substructuring methods for the model reduction of linear finite element (FE) models has its origins in the 1960s with the works of Hurty [36, 37] and Craig and Bampton [23]. A substructure is obtained by projecting the dynamics of a linear FE model onto a lower dimensional subspace spanned by so-called component modes. The component modes may be a collection of different kinds of modes such as rigid body, normal and constraint modes. The constraint modes are similar to the modes identifiable in the Guyan-Irons reduction method [31]. In general, substructuring techniques differ in the types of modes used in the component mode reduction basis. A detailed overview of the now-available substructure methods and their differences can be found in $[24,46,58]$. Substructure methods are often employed for describing elastic deformations in multibody systems as the physical meaning of the nodal coordinates on the boundaries or interface surfaces are retained. This allows easy interfacing with other components. An alternative approach to the use of substructure methods is presented by Lehner and Eberhard [48, 49]. Here Krylov-subspace based projection methods are used to obtain an input-output model that preserves the dynamical properties in a predefined frequency range.

The analysis and simulation of mechanisms with complex-shaped components that undergo large rigid body motions and small elastic deformations is unavoidably costly in terms of computational time when using classical non-linear FE approaches. Therefore, several research groups have adopted substructuring methods in their multibody code to attain better performance while retaining a good numerical accuracy. To include substructures in a multibody environment, the floating frame of reference approach is often employed $[4,45,48,49,59,60,71$, $72,73]$. Here the rigid body motion of the component is described by absolute coordinates which define the position and orientation of the floating frame. The small elastic deformations, described by relative coordinates, are superimposed onto the rigid body motion. Shabana et al. $[4,59,60]$ propose the use of substructure shape functions or shape vectors for the modelling of the elastic deformations. The approach using shape vectors is based on a lumped mass technique similar to the method presented by Haug et al. [71, 72, 73]. In the case of substructure shape functions, the more accurate consistent mass formulation can be used. However, the determination of such shape functions requires using finite elements embedded in the multibody program for the modelling of the complex-shaped component. In the approach developed by Cardona et al. [19, 20, 21], a substructure can directly be included in a multibody analysis regardless of the FE software that is used to create 
the substructure. The only inputs are the reduced mass and stiffness matrices of the substructure. The formulation is based on an approximation of the kinetic energy in terms of absolute nodal velocities expressed in a co-rotational frame. Bauchau and Rodriguez [8] used this approach for their formulation of modal based elements, where any type of modal basis can be chosen. Common to these methods is that substructure models with an arbitrary number of interface surfaces for the connection to other components can be considered.

The focus of this paper is to develop an efficient modelling approach allowing arbitrary rigid body motion and small elastic deformations of complex-shaped flexible members that can be characterized by two interface surfaces. Such components are quite common in robotics and high precision mechanisms [27], e.g. robot arms or complex-shaped flexible members. For accurate dynamic simulations, correct inclusion of their physical properties in a multibody model is paramount. To achieve this, we adopt the kinematic model of a non-linear beam element proposed in [38]. By combining this kinematic model with a substructure method for the determination of the mass and stiffness properties, a so-called non-linear two-node superelement is obtained.

In the kinematic model of the non-linear beam element, absolute nodal position and orientation coordinates, in the form of Euler parameters, are used to describe the configuration of the element in an inertial reference frame. For describing the elastic deformations, the concept of specifying independent deformations modes is employed [38]. The independent deformations modes are defined in a co-rotational frame, which allows the use of linear stiffness models for the computation of the stresses, provided that the elastic deformations remain small. This formulation appears to be very advantageous when dealing with the modelling of flexible multibody systems that may consist of elastic and rigid components [43]. The condition of rigidity can directly be accounted for by prescribing the associated deformations to be zero. For example, this allows for a direct suppression of the high frequency elongation mode of the element. Furthermore, element interconnectivity can be realized by elements sharing their absolute nodal coordinates circumventing the need to add additional algebraic constraints to the equations of motion. The non-linear two-node superelement inherits these properties by using the kinematic model of this non-linear beam element.

The substructure mass and stiffness matrices are obtained by applying the constraint modes of the Craig-Bampton method to a linear FE model created with commercially available FE software. The resulting substructure will be referred to as a linear two-node superelement. The potential and kinetic energy expressions of the linear two-node superelement are used as a basis for the determination of the potential and kinetic energy expressions of the non-linear two-node superelement. From the potential and kinetic energy expressions, the equivalent mass and stiffness matrices of the non-linear two-node superelement are determined. Subsequently, by evaluating Lagrange's equations, the internal and external forces acting on the non-linear superelement are derived. Specific care has to be taken for the determination of the kinetic energy of the non-linear two-node superelement. This requires expressing the absolute nodal velocities in a co-rotational frame similar to 
the approach of Cardona [21]. The transformation of the absolute nodal velocities can be achieved in different ways and influences the accuracy of the kinetic energy estimation.

The derivation is based on the assumption of infinitesimal elastic deformations in a co-rotational frame, though accurate results can still be obtained for small elastic deformations. The interfaces surfaces to which other components can be attached are considered to be rigid. Furthermore, it is assumed that the constraint modes are sufficient for describing the lower vibrational modes in dynamic analysis. This is often the case for the first vibrational bending modes of flexible members. Increased accuracy in dynamic analysis can be realized by including the fixed-interface normal modes of the Craig-Bampton method. However, the focus of this paper is on determining a suitable approach for the estimation of the kinetic energy which is dependent on the transformation of the absolute nodal velocities that are directly linked with the constraint modes. Therefore, other modes like the fixed-interface normal modes, which are non-essential to the transformation of the absolute nodal velocities, are not considered. With these assumptions, slender complex-shaped flexible members can be adequately modelled, as well as a variety of other components where two rigid interface surfaces can be defined.

The contents of this paper are organized as follows. In Section 2.2 the linear two-node superelement is derived and expressions for its potential and kinetic energy are given. Section 2.3 establishes the formulation of the non-linear two-node superelement. The method is validated in Section 2.4, where two spinning beam examples and an unbalanced rotating shaft example are presented.

\subsection{Linear two-node superelement}

\subsubsection{Boundary coordinates and constraint modes}

Fig. 2.1 shows a linear FE model of a flexible complex-shaped component, which is obtainable with FE software such as ANSYS ${ }^{\circledR}$. In order to construct a linear two-node superelement, boundary nodes $p$ and $q$ are introduced at either side of the component. In the initial undeformed configuration, the location of the coordinate system, with base vectors $\overline{\boldsymbol{e}}_{x}, \overline{\boldsymbol{e}}_{y}$ and $\overline{\boldsymbol{e}}_{z}$, is specified to coincide with node $p$ with its $x$-axis pointing toward node $q$. The orientation of the coordinate system around the $x$-axis can be chosen arbitrarily. ${ }^{1}$

Finite element models of complex-shaped components are commonly created using solid elements which have only translational degrees of freedom. To be able to load a single boundary node by both forces and moments, both translational and rotational coordinates are required in the superelement formalism. To achieve this for a $\mathrm{FE}$ model with only translational degrees of freedom, the nodes on the interface surfaces are rigidly attached to the boundary nodes, see Fig. 2.1. The displacement and orientation of the boundary nodes then prescribe the displacements of the nodes on

\footnotetext{
${ }^{1}$ Subsequently, the overbar will denote a kinematic quantity related to the coordinates system $\left[\overline{\boldsymbol{e}}_{x}, \overline{\boldsymbol{e}}_{y}, \overline{\boldsymbol{e}}_{z}\right]$.
} 


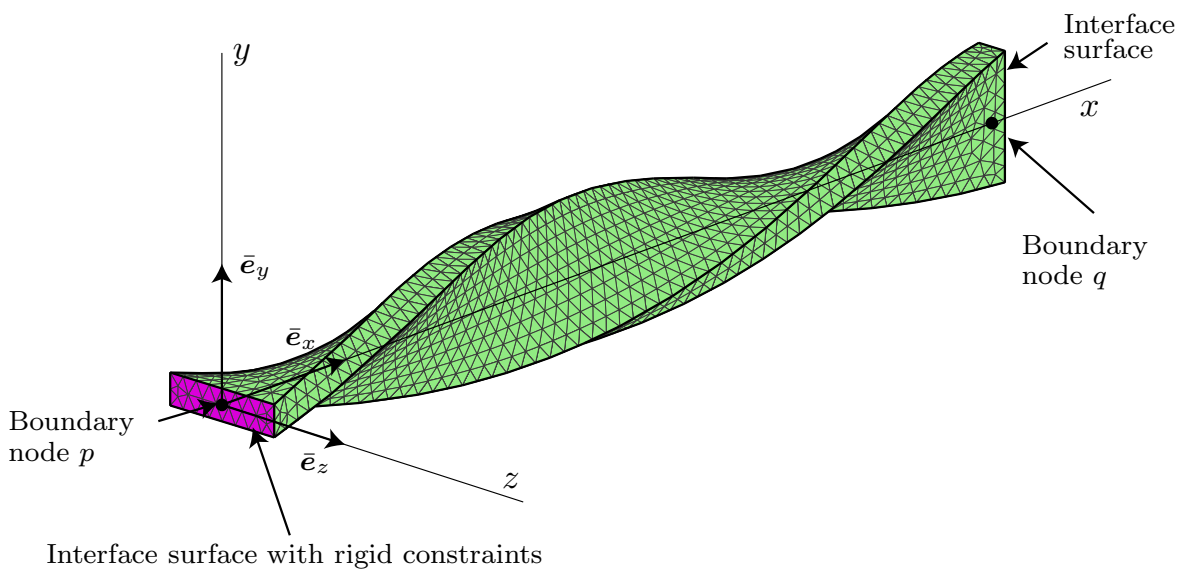

Figure 2.1: Finite element model of a flexible complex-shaped component.

the interface surfaces. The boundary node displacements and rotations are collected in the vector of boundary coordinates $\overline{\boldsymbol{\mu}}$,

$$
\overline{\boldsymbol{\mu}}=\left\{\begin{array}{lll}
\overline{\boldsymbol{u}}^{p \mathrm{~T}}, & \bar{\varphi}^{p \mathrm{~T}}, \quad \overline{\boldsymbol{u}}^{q \mathrm{~T}}, \quad \bar{\varphi}^{q \mathrm{~T}}
\end{array}\right\}^{\mathrm{T}},
$$

where $\overline{\boldsymbol{u}}^{p}, \bar{\varphi}^{p}, \overline{\boldsymbol{u}}^{q}$ and $\overline{\boldsymbol{\varphi}}^{q}$ are the nodal displacement and rotation vectors of boundary nodes $p$ and $q$, respectively.

By means of the constraint modes of the Craig-Bampton component mode method [23], the nodal displacements $\overline{\boldsymbol{u}}$ of the linear FE model can be expressed in terms of the boundary coordinates $\bar{\mu}$,

$$
\overline{\boldsymbol{u}}=\boldsymbol{V} \overline{\boldsymbol{\mu}}
$$

where $\boldsymbol{V}$ is the matrix of constraint modes. A constraint mode is defined as the static deformation of a component generated by applying a unit displacement on a boundary coordinate while fixing all other boundary coordinates [24]. The resulting set of constraint modes spans the static response of the component to loads applied on the boundary nodes. Note that a correct static response is only obtained if the interface surfaces can be considered rigid. The two-node superelement has a total of twelve constraint modes, three rotational and three translational modes at either side, see Fig. 2.2.

\subsubsection{Potential energy}

It should be noted that the constraint modes implicitly contain all six rigid body modes. For example, $\boldsymbol{V}_{1}+\boldsymbol{V}_{7}$ results in a rigid body translation in the $x$-direction. With similar linear combinations of the constraint modes, all rigid body movements can be described. However, for the determination of the potential energy only the 


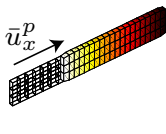

$V_{1}$

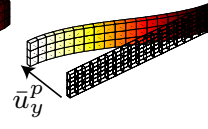

$V_{2}$

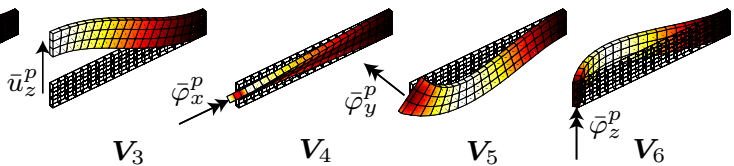

$V_{3}$

$V_{4}$

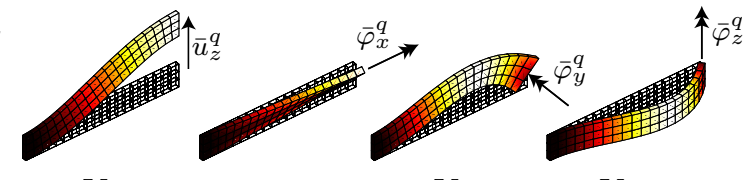

$V_{9}$

$V_{10}$

$V_{11}$

$V_{12}$

$V_{8}$ $\bigwedge^{y} \overbrace{x}^{V_{7}}$

Figure 2.2: The twelve constraint modes of a FE model of a beam, as they appear column-wise in the matrix $\boldsymbol{V}$ with associated boundary coordinates.

contributions of the elastic deformations are needed. A 6-D subspace of the constraint mode matrix $\boldsymbol{V}$ spans the space associated with purely elastic deformations. The choice of base vectors for this subspace is not unique. A possible choice consists of the constraint modes belonging to the elongation $\boldsymbol{V}_{7}$, torsion $\boldsymbol{V}_{10}$, and the four bending modes $\boldsymbol{V}_{5}, \boldsymbol{V}_{11}$ and $\boldsymbol{V}_{6}, \boldsymbol{V}_{12}$. The vector of elastic nodal displacements, $\overline{\boldsymbol{u}}_{\mathrm{e}}$, of the linear FE model can then be expressed as

$$
\overline{\boldsymbol{u}}_{\mathrm{e}}=\boldsymbol{V}_{\mathrm{e}} \overline{\boldsymbol{\mu}}_{\mathrm{e}}
$$

where

$$
\boldsymbol{V}_{\mathrm{e}}=\left[\begin{array}{llllll}
\boldsymbol{V}_{7}, & \boldsymbol{V}_{10}, & \boldsymbol{V}_{5}, & \boldsymbol{V}_{11}, & \boldsymbol{V}_{6}, & \boldsymbol{V}_{12}
\end{array}\right]
$$

and

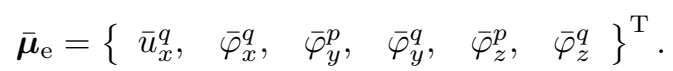

Here $\boldsymbol{V}_{\mathrm{e}}$ is the matrix containing the deformation modes with associated deformation boundary coordinates $\overline{\boldsymbol{\mu}}_{\mathrm{e}}$.

With Eq. (2.3a) the potential energy, $P$, of the superelement can be expressed in terms of the elastic boundary coordinates $\overline{\boldsymbol{\mu}}_{\mathrm{e}}$,

$$
P=\frac{1}{2} \overline{\boldsymbol{u}}_{\mathrm{e}}^{\mathrm{T}} \overline{\boldsymbol{K}}_{\mathrm{FEM}} \overline{\boldsymbol{u}}_{\mathrm{e}}=\frac{1}{2} \overline{\boldsymbol{\mu}}_{\mathrm{e}}^{\mathrm{T}} \boldsymbol{V}_{\mathrm{e}}^{\mathrm{T}} \overline{\boldsymbol{K}}_{\mathrm{FEM}} \boldsymbol{V}_{\mathrm{e}} \overline{\boldsymbol{\mu}}_{\mathrm{e}}
$$

where $\overline{\boldsymbol{K}}_{\mathrm{FEM}}$ is the stiffness matrix of the linear FE model. From Eq. (2.4), we can define the $6 \times 6$ reduced stiffness matrix $\overline{\boldsymbol{S}}$ as

$$
\overline{\boldsymbol{S}}=\boldsymbol{V}_{\mathrm{e}}^{\mathrm{T}} \overline{\boldsymbol{K}}_{\mathrm{FEM}} \boldsymbol{V}_{\mathrm{e}}
$$

\subsubsection{Kinetic energy}

To determine the expression for the kinetic energy, Eq. (2.2) is differentiated with respect to time, yielding

$$
\dot{\bar{u}}=V \dot{\bar{\mu}}=V \bar{v}
$$


where

$$
\overline{\boldsymbol{v}}=\left\{\begin{array}{llll}
\overline{\boldsymbol{v}}^{p \mathrm{~T}}, & \overline{\boldsymbol{\omega}}^{p \mathrm{~T}}, & \overline{\boldsymbol{v}}^{q \mathrm{~T}}, & \overline{\boldsymbol{\omega}}^{q \mathrm{~T}}
\end{array}\right\}^{\mathrm{T}} .
$$

Here $\overline{\boldsymbol{v}}$ is the vector of absolute boundary velocities containing the boundary node translational velocities, $\overline{\boldsymbol{v}}^{p}$ and $\overline{\boldsymbol{v}}^{q}$, and angular velocities, $\overline{\boldsymbol{\omega}}^{p}$ and $\overline{\boldsymbol{\omega}}^{q}$, of nodes $p$ and $q$, respectively. Their components are expressed along the base vectors $\overline{\boldsymbol{e}}_{x}, \overline{\boldsymbol{e}}_{y}$ and $\overline{\boldsymbol{e}}_{z}$. The kinetic energy, $T$, can be expressed as

$$
T=\frac{1}{2} \dot{\overline{\boldsymbol{u}}}^{\mathrm{T}} \overline{\boldsymbol{M}}_{\mathrm{FEM}} \dot{\overline{\boldsymbol{u}}}=\frac{1}{2} \overline{\boldsymbol{v}}^{\mathrm{T}} \boldsymbol{V}^{\mathrm{T}} \overline{\boldsymbol{M}}_{\mathrm{FEM}} \boldsymbol{V} \overline{\boldsymbol{v}},
$$

where $\overline{\boldsymbol{M}}_{\mathrm{FEM}}$ is the mass matrix of the linear FE model. From Eq. (2.7), we can define the $12 \times 12$ reduced mass matrix $\bar{M}$ as

$$
\overline{\boldsymbol{M}}=\boldsymbol{V}^{\mathrm{T}} \overline{\boldsymbol{M}}_{\mathrm{FEM}} \boldsymbol{V} .
$$

\subsection{Non-linear two-node superelement}

The linear two-node superelement cannot be used to describe large rigid body rotations due to the linear approximation of rotations in its kinematic model. To obtain a non-linear two-node superelement capable of large rigid body rotations and small local deformations, a kinematic model is required that correctly describes the element's configuration in 3-D space when undergoing large rigid body rotations. The first part of this section describes the kinematic model that is used for this purpose. Then in the second and third part, using the expressions of the potential and kinetic energy of the linear two-node superelement, we derive expressions for the potential and kinetic energy of the non-linear two-node superelement. Its dynamic relations can then be derived using Lagrange's equations.

\subsubsection{Nodal coordinates and deformation modes}

Identical to the non-linear beam element introduced in [38], the configuration of the non-linear two-node superelement is defined by the position vectors $\boldsymbol{r}^{p}$ and $\boldsymbol{r}^{q}$ and the orientation of the orthonormal triads, $\left[\boldsymbol{e}_{x}^{p}, \boldsymbol{e}_{y}^{p}, \boldsymbol{e}_{z}^{p}\right]$ and $\left[\boldsymbol{e}_{x}^{q}, \boldsymbol{e}_{y}^{q}, \boldsymbol{e}_{z}^{q}\right]$ rigidly attached to the nodes $p$ and $q$, see Fig. 2.3. In the initial undeflected state, the triads coincide with the initial orientation described by the rotation matrix $\boldsymbol{R}_{0}$. In a deflected state, the orientation of the triads can be computed from the rotation matrices $\boldsymbol{R}^{p}$ and $\boldsymbol{R}^{q}$, which describe the orientation of nodes $p$ and $q$ with respect to the initial orientation,

$$
\begin{aligned}
{\left[\begin{array}{lll}
\boldsymbol{e}_{x}^{p}, & \boldsymbol{e}_{y}^{p}, & \boldsymbol{e}_{z}^{p}
\end{array}\right] } & =\boldsymbol{R}^{p} \boldsymbol{R}_{0}\left[\begin{array}{lll}
\boldsymbol{n}_{x}, & \boldsymbol{n}_{y}, & \boldsymbol{n}_{z}
\end{array}\right], \\
{\left[\begin{array}{lll}
\boldsymbol{e}_{x}^{q}, & \boldsymbol{e}_{y}^{q}, & \boldsymbol{e}_{z}^{q}
\end{array}\right] } & =\boldsymbol{R}^{q} \boldsymbol{R}_{0}\left[\begin{array}{lll}
\boldsymbol{n}_{x}, & \boldsymbol{n}_{y}, & \boldsymbol{n}_{z}
\end{array}\right] .
\end{aligned}
$$

Here $\boldsymbol{n}_{x}, \boldsymbol{n}_{y}$ and $\boldsymbol{n}_{z}$ are the unit vectors of the inertial reference frame. The initial orientation $\boldsymbol{R}_{0}$ is used such that in the assembly of the finite elements the rotation coordinates that parametrize rotation matrices $\boldsymbol{R}^{p}$ and $\boldsymbol{R}^{q}$ can be shared by multiple elements. The parametrization of rotations can be accomplished in several ways, such 


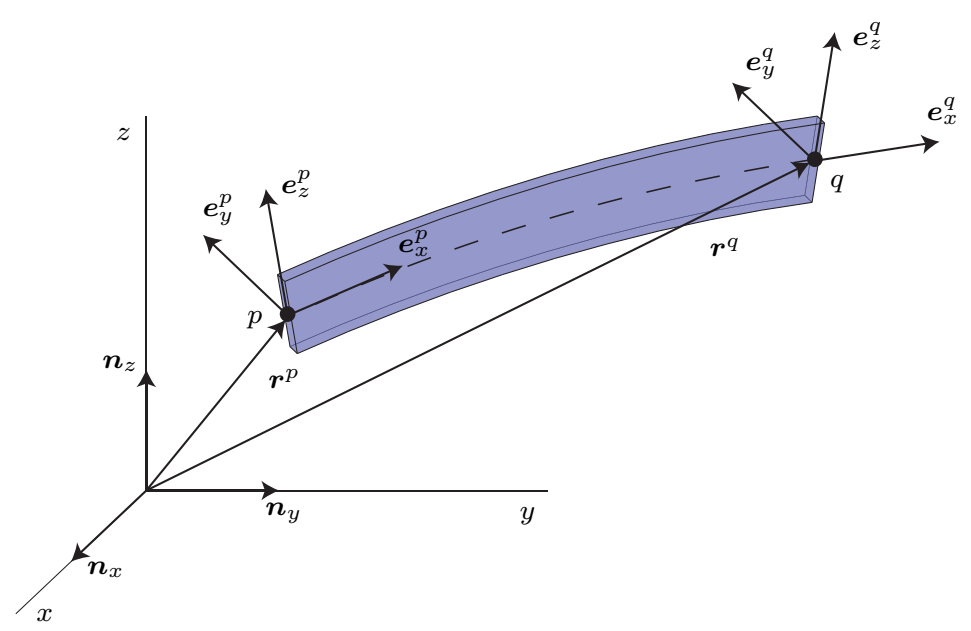

Figure 2.3: The configuration of the non-linear two-node superelement expressed in the inertial reference frame.

as by modified Euler angles, Euler parameters, Rodrigues parameters or the Cartesian rotation vector [28]. In this paper we use Euler parameters for the parametrization of rotations to avoid singularity problems associated with Euler angles and to avoid the use of trigonometric functions. Instead, the components of a rotation matrix $\boldsymbol{R}$, parametrized with Euler parameters, are computationally efficient quadratic algebraic expressions [38].

We now have as nodal coordinates of the non-linear two-node superelement two sets of Cartesian coordinates, $\boldsymbol{r}^{p}$ and $\boldsymbol{r}^{q}$, describing the nodal positions in the inertial coordinate system and two sets of Euler parameters, $\boldsymbol{\lambda}^{p}$ and $\boldsymbol{\lambda}^{q}$, representing the orientation of the triads $\left[\boldsymbol{e}_{x}^{p}, \boldsymbol{e}_{y}^{p}, \boldsymbol{e}_{z}^{p}\right]$ and $\left[\boldsymbol{e}_{x}^{q}, \boldsymbol{e}_{y}^{q}, \boldsymbol{e}_{z}^{q}\right]$ at nodes $p$ and $q$. The vector $\boldsymbol{x}$ of nodal coordinates can then be written as

$$
\boldsymbol{x}=\left\{\begin{array}{llll}
\boldsymbol{r}^{p \mathrm{~T}}, & \boldsymbol{\lambda}^{p \mathrm{~T}}, & \boldsymbol{r}^{q \mathrm{~T}}, & \boldsymbol{\lambda}^{q \mathrm{~T}}
\end{array}\right\}^{\mathrm{T}} .
$$

Note that the parametrization of rotations is redundant due to the constraint $\boldsymbol{\lambda}^{\mathrm{T}} \boldsymbol{\lambda}=$ 1, imposed on the Euler parameters of Eq. (2.10). This results in a total of twelve independent nodal coordinates.

The time derivatives of Eq. (2.10) are

$$
\dot{\boldsymbol{x}}=\left\{\dot{\boldsymbol{r}}^{p \mathrm{~T}}, \quad \dot{\boldsymbol{\lambda}}^{p \mathrm{~T}}, \quad \dot{\boldsymbol{r}}^{q \mathrm{~T}}, \quad \dot{\boldsymbol{\lambda}}^{q \mathrm{~T}}\right\}^{\mathrm{T}} .
$$

The $14 \times 1$ vector $\dot{\boldsymbol{x}}$ can be related with the $12 \times 1$ absolute nodal velocity vector $\boldsymbol{v}$ by

$$
\boldsymbol{v}=\left\{\begin{array}{c}
\boldsymbol{v}^{p} \\
\boldsymbol{\omega}^{p} \\
\boldsymbol{v}^{q} \\
\boldsymbol{\omega}^{q}
\end{array}\right\}=\left[\begin{array}{llll}
\boldsymbol{I} & & & \\
& 2 \boldsymbol{\Lambda}^{p} & & \\
& & \boldsymbol{I} & \\
& & & 2 \boldsymbol{\Lambda}^{q}
\end{array}\right]\left\{\begin{array}{c}
\dot{\boldsymbol{r}}^{p} \\
\dot{\boldsymbol{\lambda}}^{p} \\
\dot{\boldsymbol{r}}^{q} \\
\dot{\boldsymbol{\lambda}}^{q}
\end{array}\right\}
$$


where $\boldsymbol{v}^{p}$ and $\boldsymbol{v}^{q}$ are the absolute nodal translational velocities and $\boldsymbol{\omega}^{p}$ and $\boldsymbol{\omega}^{q}$ are the absolute nodal angular velocities respectively defined by

$$
\boldsymbol{\omega}^{p}=2 \boldsymbol{\Lambda}^{p} \dot{\boldsymbol{\lambda}}^{p} \quad \text { and } \quad \boldsymbol{\omega}^{q}=2 \boldsymbol{\Lambda}^{q} \dot{\boldsymbol{\lambda}}^{q} .
$$

Here the matrices $\boldsymbol{\Lambda}^{p}$ and $\boldsymbol{\Lambda}^{q}$ are defined by the Euler parameters $\boldsymbol{\lambda}^{p}$ and $\boldsymbol{\lambda}^{q}$ and are of the following form:

$$
\boldsymbol{\Lambda}=\left[\begin{array}{rrrr}
-\lambda_{1} & \lambda_{0} & -\lambda_{3} & \lambda_{2} \\
-\lambda_{2} & \lambda_{3} & \lambda_{0} & -\lambda_{1} \\
-\lambda_{3} & -\lambda_{2} & \lambda_{1} & \lambda_{0}
\end{array}\right]
$$

with $\lambda_{0}, \lambda_{1}, \lambda_{2}$ and $\lambda_{3}$ being the four Euler parameters of a vector $\boldsymbol{\lambda}$.

To determine the elastic deformations of the non-linear two-node superelement from the configuration given by the nodal coordinate vector $\boldsymbol{x}$, we employ the concept of specifying independent deformation modes from the non-linear beam element model $[38,43]$. As the superelement has twelve independent nodal coordinates and six rigid body modes, six independent deformation modes, specified by a set of deformation coordinates, $\varepsilon$, can be expressed as analytical functions of the nodal coordinate vector $\boldsymbol{x}$ and the reference length $l_{0}[43]$,

$$
\varepsilon=\mathcal{D}(\boldsymbol{x}),
$$

where

$$
\begin{aligned}
& \varepsilon_{1}=l-l_{0}, \\
& \varepsilon_{2}=l_{0}\left(\boldsymbol{e}_{z}^{p} \cdot \boldsymbol{e}_{y}^{q}-\boldsymbol{e}_{y}^{p} \cdot \boldsymbol{e}_{z}^{q}\right) / 2, \\
& \varepsilon_{3}=-l_{0} \boldsymbol{e}_{l} \cdot \boldsymbol{e}_{z}^{p}, \\
& \varepsilon_{4}=l_{0} \boldsymbol{e}_{l} \cdot \boldsymbol{e}_{z}^{q}, \\
& \varepsilon_{5}=l_{0} \boldsymbol{e}_{l} \cdot \boldsymbol{e}_{y}^{p}, \\
& \varepsilon_{6}=-l_{0} \boldsymbol{e}_{l} \cdot \boldsymbol{e}_{y}^{q},
\end{aligned}
$$

with

$$
l=\left\|\boldsymbol{r}^{q}-\boldsymbol{r}^{p}\right\| \quad \text { and } \quad \boldsymbol{e}_{l}=\left(\boldsymbol{r}^{q}-\boldsymbol{r}^{p}\right) / l
$$

Here $\boldsymbol{e}_{y}^{p}, \boldsymbol{e}_{z}^{p}, \boldsymbol{e}_{y}^{q}$ and $\boldsymbol{e}_{z}^{q}$ are defined by Eq. (2.9). The deformation coordinates represent a set of deformation modes, where the first deformation coordinate $\varepsilon_{1}$, describes the elongation of the element, the second one, $\varepsilon_{2}$, describes the torsion, and the remaining coordinates are associated with the bending deformations. The deformation modes are visualized in Fig. 2.4. The definitions of the deformation coordinates are invariant under arbitrary rigid body motion of the superelement. We note that the method outlined here is not particularly restricted to the set of deformation coordinates given above. Other descriptions that describe six independent deformation modes in terms of the absolute nodal coordinates could be used as well. 

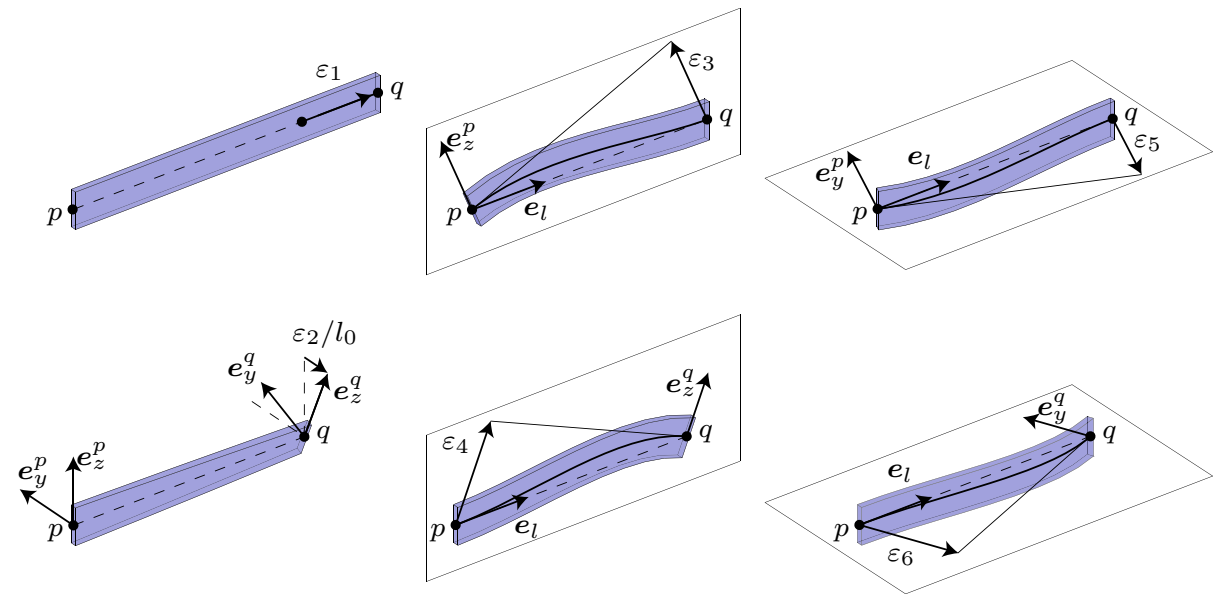

Figure 2.4: Graphical representation of the deformation modes with deformation coordinates $\varepsilon_{1}$ through $\varepsilon_{6}$.

\subsubsection{Potential energy}

\section{Relating deformation coordinates}

With an expression for the potential energy of the non-linear two-node superelement, the internal forces acting on the superelement can be determined. The potential energy can be expressed in terms of the deformation coordinates $\varepsilon$,

$$
P=\frac{1}{2} \varepsilon^{\mathrm{T}} \boldsymbol{S} \boldsymbol{\varepsilon} .
$$

Here $\boldsymbol{S}$ is the $6 \times 6$ equivalent stiffness matrix of the non-linear two-node superelement.

An expression for $\boldsymbol{S}$ can be obtained by relating Eq. (2.16) with the potential energy of the linear two-node superelement given by Eq. (2.4). This requires relating the deformation coordinates $\varepsilon$ with the deformation boundary coordinates $\overline{\boldsymbol{\mu}}_{\mathrm{e}}$. From Fig. 2.2 and Fig. 2.4, it can be verified that when considering infinitesimal elastic deformations, this relation is given by

$$
\overline{\boldsymbol{\mu}}_{\mathrm{e}}=\boldsymbol{A} \varepsilon,
$$

where

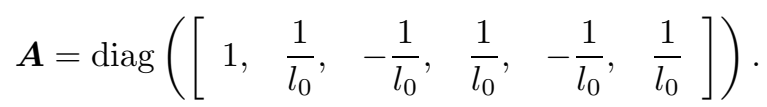

The matrix $\boldsymbol{A}$ is diagonal due to the choice of base vectors in Eq. (2.3).

\section{Equivalent stiffness matrix}

Substituting Eqs. (2.17a) and (2.5) in Eq. (2.4) and equating the result with Eq. (2.16) yields

$$
P=\frac{1}{2} \varepsilon^{\mathrm{T}} \boldsymbol{A}^{\mathrm{T}} \overline{\boldsymbol{S}} \boldsymbol{A} \boldsymbol{\varepsilon}=\frac{1}{2} \varepsilon^{\mathrm{T}} \boldsymbol{S} \boldsymbol{\varepsilon} .
$$


From Eq. (2.18) the $6 \times 6$ equivalent stiffness matrix $\boldsymbol{S}$ can be identified as

$$
\boldsymbol{S}=\boldsymbol{A}^{\mathrm{T}} \overline{\boldsymbol{S}} \boldsymbol{A} .
$$

The stiffness matrix $\boldsymbol{S}$ remains constant during a simulation. Note that the stiffness matrix $\overline{\boldsymbol{S}}$ of the linear two-node superelement (see Eq. 2.5) can directly be derived from a FE model created with FE software such as ANSYs.

\subsubsection{Kinetic energy}

\section{Relating velocities}

With an expression for the kinetic energy of the non-linear two-node superelement, the external forces acting on the superelement can be determined. The kinetic energy can be expressed in terms of the nodal coordinate time derivatives $\dot{\boldsymbol{x}}$,

$$
T=\frac{1}{2} \dot{\boldsymbol{x}}^{\mathrm{T}} \boldsymbol{M} \dot{\boldsymbol{x}},
$$

where $\boldsymbol{M}$ is the $14 \times 14$ equivalent mass matrix of the non-linear two-node superelement.

Similar to the approach used in Section 2.3.2, an expression for the equivalent mass matrix can be obtained by relating the kinetic energy of Eq. (2.20) with the kinetic energy of the linear two-node superelement given by Eq. (2.7). This requires relating the time derivatives of the nodal coordinates, $\dot{\boldsymbol{x}}$, with the absolute boundary velocities $\overline{\boldsymbol{v}}$ whose components are expressed along the base vectors $\overline{\boldsymbol{e}}_{x}, \overline{\boldsymbol{e}}_{y}$ and $\overline{\boldsymbol{e}}_{z}$, see Fig. 2.1. To determine this relation we will reintroduce the coordinate system $\left[\overline{\boldsymbol{e}}_{x}, \overline{\boldsymbol{e}}_{y}, \overline{\boldsymbol{e}}_{z}\right]$ from the linear superelement in Fig. 2.1 as the co-rotational frame of the non-linear superelement. Furthermore, with Eq. (2.12), we already have the transformation between the vector $\dot{\boldsymbol{x}}$ and the absolute nodal velocities $\boldsymbol{v}$ expressed in the inertial reference frame. Then, by expressing the absolute nodal velocities $\boldsymbol{v}$ in the co-rotational frame $\left[\overline{\boldsymbol{e}}_{x}, \overline{\boldsymbol{e}}_{y}, \overline{\boldsymbol{e}}_{z}\right]$ we obtain the vector $\overline{\boldsymbol{v}}$.

We will investigate three approaches for expressing the absolute velocities in the co-rotational frame. They are visualized in Fig. 2.5 for a deflected beam which has a rolling support on the left and is clamped on the right. The first approach is a transformation with a node-attached reference frame, see Fig. 2.5(a). Secondly, a transformation with an average orientation reference frame is considered, see Fig. 2.5(b). Thirdly, we investigate an approach inspired by the method proposed by Cardona [21]. Here the angular velocities are expressed in the coordinate systems attached to the superelement nodes while the translational velocities are expressed in the average orientation reference frame, see Fig. 2.5(c). Each of the approaches combined with Eq. (2.12) yields a $12 \times 14$ velocity transformation matrix $\boldsymbol{B}_{i}$ which relates the vectors $\overline{\boldsymbol{v}}$ and $\dot{\boldsymbol{x}}$,

$$
\overline{\boldsymbol{v}}=\boldsymbol{B}_{i} \dot{\boldsymbol{x}},
$$

where the subscript $i=1,2$ or 3 denotes which velocity transformation matrix is considered for the transformation of the velocities. 


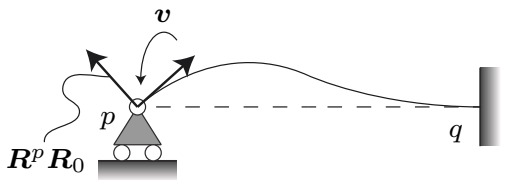

(a) Node-attached reference frame $\boldsymbol{R}^{p} \boldsymbol{R}_{0}$ at node $p$, resulting in velocity transformation matrix $\boldsymbol{B}_{1}$

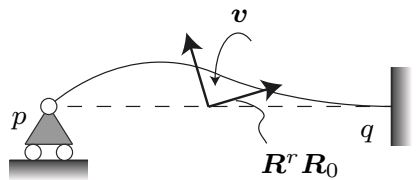

(b) Average orientation reference frame $\boldsymbol{R}^{r} \boldsymbol{R}_{0}$, resulting in velocity transformation matrix $\boldsymbol{B}_{2}$

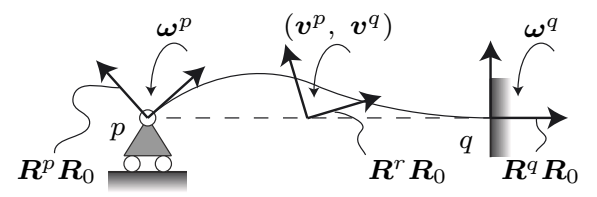

(c) Angular velocities expressed in the reference frames attached to the nodes, resulting in velocity transformation matrix $\boldsymbol{B}_{3}$

Figure 2.5: A deflected beam with a rolling support on the left and clamped on the right, showing the three considered options for transforming the nodal velocity vector $\boldsymbol{v}$.

Node-attached reference frame In the first approach, the reference frame is attached to node $p$. Then we have for the orientation of the co-rotational frame in the inertial reference frame

$$
\left[\begin{array}{lll}
\overline{\boldsymbol{e}}_{x}, & \overline{\boldsymbol{e}}_{y}, & \overline{\boldsymbol{e}}_{z}
\end{array}\right]=\boldsymbol{R}^{p} \boldsymbol{R}_{0}\left[\begin{array}{lll}
\boldsymbol{n}_{x}, & \boldsymbol{n}_{y}, & \boldsymbol{n}_{z}
\end{array}\right] .
$$

Expressing the nodal translational and angular velocities of vector $\boldsymbol{v}$ in this nodeattached reference frame gives

$$
\left\{\begin{array}{c}
\overline{\boldsymbol{v}}^{p} \\
\overline{\boldsymbol{\omega}}^{p} \\
\overline{\boldsymbol{v}}^{q} \\
\overline{\boldsymbol{\omega}}^{q}
\end{array}\right\}=\left[\begin{array}{cccc}
\boldsymbol{R}_{0}^{\mathrm{T}} \boldsymbol{R}^{p \mathrm{~T}} & & & \\
& \boldsymbol{R}_{0}^{\mathrm{T}} \boldsymbol{R}^{p \mathrm{~T}} & & \\
& & \boldsymbol{R}_{0}^{\mathrm{T}} \boldsymbol{R}^{p \mathrm{~T}} & \\
& & & \boldsymbol{R}_{0}^{\mathrm{T}} \boldsymbol{R}^{p \mathrm{~T}}
\end{array}\right]\left\{\begin{array}{c}
\boldsymbol{v}^{p} \\
\boldsymbol{\omega}^{p} \\
\boldsymbol{v}^{q} \\
\boldsymbol{\omega}^{q}
\end{array}\right\} .
$$

By substituting Eq. (2.12) in Eq. (2.23), we obtain the $12 \times 14$ velocity transformation matrix $\boldsymbol{B}_{1}$ relating the vectors $\overline{\boldsymbol{v}}$ and $\dot{\boldsymbol{x}}$,

$$
\boldsymbol{B}_{1}=\left[\begin{array}{cccc}
\boldsymbol{R}_{0}^{\mathrm{T}} \boldsymbol{R}^{p \mathrm{~T}} & & & \\
& 2 \boldsymbol{R}_{0}^{\mathrm{T}} \boldsymbol{R}^{p \mathrm{~T}} \boldsymbol{\Lambda}^{p} & & \\
& & \boldsymbol{R}_{0}^{\mathrm{T}} \boldsymbol{R}^{p \mathrm{~T}} & \\
& & & 2 \boldsymbol{R}_{0}^{\mathrm{T}} \boldsymbol{R}^{p \mathrm{~T}} \boldsymbol{\Lambda}^{q}
\end{array}\right] .
$$

To investigate the suitability of Eq. (2.23) for the computation of the kinetic energy in Eq. (2.7), we note that the kinetic energy is in fact dependent on the absolute velocities of all nodes in the FE model. By substituting Eq. (2.23) in Eq. (2.6a) and 
considering infinitesimal elastic deformations, we can describe the absolute velocity of the $i^{\text {th }}$ node of the FE model, $\dot{\overline{\boldsymbol{u}}}^{(i)}$, expressed in the node-attached reference frame, as

$$
\begin{aligned}
\dot{\overline{\boldsymbol{u}}}^{(i)}= & {\left[\boldsymbol{V}_{1}^{(i)}, \boldsymbol{V}_{2}^{(i)}, \boldsymbol{V}_{3}^{(i)}\right] \boldsymbol{R}_{0}^{\mathrm{T}} \boldsymbol{R}^{p \mathrm{~T}} \boldsymbol{v}^{p}+\left[\boldsymbol{V}_{4}^{(i)}, \boldsymbol{V}_{5}^{(i)}, \boldsymbol{V}_{6}^{(i)}\right] \boldsymbol{R}_{0}^{\mathrm{T}} \boldsymbol{R}^{p \mathrm{~T}} \boldsymbol{\omega}^{p} } \\
+ & {\left[\boldsymbol{V}_{7}^{(i)}, \boldsymbol{V}_{8}^{(i)}, \boldsymbol{V}_{9}^{(i)}\right] \boldsymbol{R}_{0}^{\mathrm{T}} \boldsymbol{R}^{p \mathrm{~T}} \boldsymbol{v}^{q}+\left[\boldsymbol{V}_{10}^{(i)}, \boldsymbol{V}_{11}^{(i)}, \boldsymbol{V}_{12}^{(i)}\right] \boldsymbol{R}_{0}^{\mathrm{T}} \boldsymbol{R}^{p \mathrm{~T}} \boldsymbol{\omega}^{q} }
\end{aligned}
$$

where $\boldsymbol{V}_{n}^{(i)}$ is the vector with the $x, y$ and $z$ contributions of the $n^{\text {th }}$ constraint mode for the $i^{\text {th }}$ node of the FE model. In Appendix A it is shown that the expression in Eq. (2.25) can be written as

$$
\dot{\overline{\boldsymbol{u}}}^{(i)}=\boldsymbol{R}_{0}^{\mathrm{T}} \boldsymbol{R}^{p \mathrm{~T}} \boldsymbol{v}^{p}+\left(\boldsymbol{R}_{0}^{\mathrm{T}} \boldsymbol{R}^{p \mathrm{~T}} \boldsymbol{\omega}^{p}\right) \times \overline{\boldsymbol{r}}_{0}^{(i)}+\dot{\overline{\boldsymbol{e}}}^{(i)},
$$

where $\overline{\boldsymbol{r}}_{0}$ is the position vector of the nodes in the FE model in undeformed configuration and $\dot{\bar{e}}$ describes the elastic deformation velocities with respect to the node-attached reference frame.

This result can be compared with the exact non-linear solution obtained by the floating frame of reference approach. Here we write for the nodal coordinates $\boldsymbol{r}$ of the FE model,

$$
\boldsymbol{r}^{(i)}=\boldsymbol{r}^{p}+\boldsymbol{R}^{p} \boldsymbol{R}_{0}\left(\overline{\boldsymbol{r}}_{0}^{(i)}+\overline{\boldsymbol{e}}^{(i)}\right),
$$

where $\overline{\boldsymbol{e}}$ describes the elastic deformations with respect to the orientation of the nodeattached reference frame. After differentiating with respect to time and expressing the resulting absolute velocities in the node-attached reference frame, we obtain

$$
\begin{aligned}
\dot{\overline{\boldsymbol{u}}}^{(i)} & =\boldsymbol{R}_{0}^{\mathrm{T}} \boldsymbol{R}^{p \mathrm{~T}} \dot{\boldsymbol{r}}^{(i)} \\
& =\boldsymbol{R}_{0}^{\mathrm{T}} \boldsymbol{R}^{p \mathrm{~T}} \boldsymbol{v}^{p}+\left(\boldsymbol{R}_{0}^{\mathrm{T}} \boldsymbol{R}^{p \mathrm{~T}} \boldsymbol{\omega}^{p}\right) \times\left(\overline{\boldsymbol{r}}_{0}^{(i)}+\overline{\boldsymbol{e}}^{(i)}\right)+\dot{\overline{\boldsymbol{e}}}^{(i)} .
\end{aligned}
$$

By comparison with Eq. (2.26), it is clear that the effects of the elastic deformations on the rigid body angular velocities are not included as we considered infinitesimal elastic deformations. The contributions of this term are expected to remain small for small elastic deformations. We note that due to the choice of reference frame at node $p$, the resulting error is largest at node $q$. Also, it can be observed that in the absence of elastic deformations, the exact rigid body solution is obtained with Eq. (2.26).

Average orientation reference frame As a second approach, to minimize the error and to give a more symmetric treatment of the velocities at nodes $p$ and $q$, we define a reference frame which is the average orientation of the triads $\left[\boldsymbol{e}_{x}^{p}, \boldsymbol{e}_{y}^{p}, \boldsymbol{e}_{z}^{p}\right]$ and $\left[\boldsymbol{e}_{x}^{q}, \boldsymbol{e}_{y}^{q}, \boldsymbol{e}_{z}^{q}\right]$ at nodes $p$ and $q$. The average orientation, $\boldsymbol{R}^{r}$, with respect to the initial orientation, $\boldsymbol{R}_{0}$, can be computed from the average set of Euler parameters $\boldsymbol{\lambda}^{r}$ defined by

$$
\boldsymbol{\lambda}^{r}=\frac{\boldsymbol{\lambda}^{p}+\boldsymbol{\lambda}^{q}}{\left\|\boldsymbol{\lambda}^{p}+\boldsymbol{\lambda}^{q}\right\|},
$$


where the differences between $\boldsymbol{\lambda}^{p}$ and $\boldsymbol{\lambda}^{q}$ are assumed to be small. Using the average orientation $\boldsymbol{R}^{r}$, the orientation of the co-rotational frame in the inertial reference frame is given by

$$
\left[\begin{array}{lll}
\overline{\boldsymbol{e}}_{x}, & \overline{\boldsymbol{e}}_{y}, & \overline{\boldsymbol{e}}_{z}
\end{array}\right]=\boldsymbol{R}^{r} \boldsymbol{R}_{0}\left[\begin{array}{lll}
\boldsymbol{n}_{x}, & \boldsymbol{n}_{y}, & \boldsymbol{n}_{z}
\end{array}\right] .
$$

Expressing the absolute velocities $\boldsymbol{v}$ in this frame and substituting Eq. (2.12) gives for the $12 \times 14$ velocity transformation matrix $\boldsymbol{B}_{2}$,

$$
\boldsymbol{B}_{2}=\left[\begin{array}{cccc}
\boldsymbol{R}_{0}^{\mathrm{T}} \boldsymbol{R}^{r \mathrm{~T}} & & & \\
& 2 \boldsymbol{R}_{0}^{\mathrm{T}} \boldsymbol{R}^{r \mathrm{~T}} \boldsymbol{\Lambda}^{p} & & \\
& & \boldsymbol{R}_{0}^{\mathrm{T}} \boldsymbol{R}^{r \mathrm{~T}} & \\
& & & 2 \boldsymbol{R}_{0}^{\mathrm{T}} \boldsymbol{R}^{r \mathrm{~T}} \boldsymbol{\Lambda}^{q}
\end{array}\right] .
$$

Average orientation and node-attached reference frames Finally, following the approach of Cardona [21], the angular velocities, $\boldsymbol{\omega}^{p}$ and $\boldsymbol{\omega}^{q}$, are respectively expressed in the reference frames attached to nodes $p$ and $q$ given by Eq. (2.9), while still using the average orientation reference frame of Eq. (2.30) to transform the translational velocities. This results in the velocity transformation matrix $\boldsymbol{B}_{3}$,

$$
\boldsymbol{B}_{3}=\left[\begin{array}{cccc}
\boldsymbol{R}_{0}^{\mathrm{T}} \boldsymbol{R}^{r \mathrm{~T}} & & & \\
& 2 \boldsymbol{R}_{0}^{\mathrm{T}} \overline{\boldsymbol{\Lambda}}^{p} & & \\
& & \boldsymbol{R}_{0}^{\mathrm{T}} \boldsymbol{R}^{r \mathrm{~T}} & \\
& & & 2 \boldsymbol{R}_{0}^{\mathrm{T}} \overline{\boldsymbol{\Lambda}}^{q}
\end{array}\right],
$$

where

$$
\overline{\boldsymbol{\Lambda}}^{p}=\boldsymbol{R}^{p \mathrm{~T}} \boldsymbol{\Lambda}^{p} \quad \text { and } \quad \overline{\boldsymbol{\Lambda}}^{q}=\boldsymbol{R}^{q \mathrm{~T}} \boldsymbol{\Lambda}^{q} .
$$

\section{Equivalent mass matrix}

Substituting Eqs. (2.21) and (2.8) in Eq. (2.7) and equating the result with Eq. (2.20) yields,

$$
T=\frac{1}{2} \dot{\boldsymbol{x}}^{\mathrm{T}} \boldsymbol{B}_{i}^{\mathrm{T}} \overline{\boldsymbol{M}} \boldsymbol{B}_{i} \dot{\boldsymbol{x}}=\frac{1}{2} \dot{\boldsymbol{x}}^{\mathrm{T}} \boldsymbol{M} \dot{\boldsymbol{x}} \quad \text { with } \quad i=1,2 \text { or } 3,
$$

from which it can be observed that the $14 \times 14$ equivalent mass matrix $M$ is given by

$$
\boldsymbol{M}=\boldsymbol{B}_{i}^{\mathrm{T}} \overline{\boldsymbol{M}} \boldsymbol{B}_{i} .
$$

Here the mass matrix $\boldsymbol{M}$ is dependent on the orientation of the co-rotational reference frame due to its dependency on the velocity transformation matrix $\boldsymbol{B}_{i}$. The matrix $\overline{\boldsymbol{M}}$ remains constant during simulation and can directly be determined from a linear FE model, see Eq. (2.8).

\subsubsection{Dynamics}

We can determine the forces acting on the superelement by considering the principle of virtual work. The virtual work done by external forces $f_{\text {ext }}$ should be equal to the 
virtual work absorbed by the inertial forces $\boldsymbol{f}_{\text {in }}$ and the generalized stress resultants $\boldsymbol{\sigma}$,

$$
\delta \boldsymbol{x}^{\mathrm{T}} \boldsymbol{f}_{\text {ext }}=-\delta \boldsymbol{x}^{\mathrm{T}} \boldsymbol{f}_{\mathrm{in}}+\delta \boldsymbol{\varepsilon}^{\mathrm{T}} \boldsymbol{\sigma}
$$

Using Lagrange's equations, the virtual work absorbed by the inertial forces and generalized stress resultants can also be expressed in terms of potential and kinetic energy,

$$
\delta \boldsymbol{x}^{\mathrm{T}} \boldsymbol{f}_{\text {ext }}=\delta \boldsymbol{x}^{\mathrm{T}}\left(\frac{d}{d t}\left(\frac{\partial T}{\partial \dot{\boldsymbol{x}}}\right)-\frac{\partial T}{\partial \boldsymbol{x}}+\frac{\partial P}{\partial \boldsymbol{x}}\right)^{\mathrm{T}} .
$$

Substituting the potential and kinetic energy expressions of Eq. (2.18) and Eq. (2.34) in Eq. (2.37) and evaluating the derivatives gives

$$
\delta \boldsymbol{x}^{\mathrm{T}} \boldsymbol{f}_{\mathrm{ext}}=\delta \boldsymbol{x}^{\mathrm{T}}\left(\boldsymbol{M} \ddot{\boldsymbol{x}}+\left(\left(\dot{\boldsymbol{B}}_{i}-\boldsymbol{B}_{i}^{*}\right)^{\mathrm{T}} \overline{\boldsymbol{M}} \boldsymbol{B}_{i}+\boldsymbol{B}_{i}^{\mathrm{T}} \overline{\boldsymbol{M}} \dot{\boldsymbol{B}}_{i}\right) \dot{\boldsymbol{x}}\right)+\delta \boldsymbol{\varepsilon}^{\mathrm{T}} \boldsymbol{S} \boldsymbol{\varepsilon},
$$

where $\boldsymbol{B}^{*}$ is defined as

$$
\boldsymbol{B}^{*}=\frac{\partial \overline{\boldsymbol{v}}}{\partial \boldsymbol{x}}
$$

Expressions for $\dot{\boldsymbol{B}}_{i}$ and $\boldsymbol{B}_{i}^{*}$ are found in Appendices B and C, respectively. By comparing Eq. (2.36) with Eq. (2.38), the inertial forces and generalized stress resultants are expressed as

$$
\begin{gathered}
\boldsymbol{f}_{\mathrm{in}}=-(\boldsymbol{M} \ddot{\boldsymbol{x}}+\boldsymbol{h}), \quad \text { with } \boldsymbol{h}=\left(\left(\dot{\boldsymbol{B}}_{i}-\boldsymbol{B}_{i}^{*}\right)^{\mathrm{T}} \overline{\boldsymbol{M}} \boldsymbol{B}_{i}+\boldsymbol{B}_{i}^{\mathrm{T}} \overline{\boldsymbol{M}} \dot{\boldsymbol{B}}_{i}\right) \dot{\boldsymbol{x}} \\
\boldsymbol{\sigma}=\boldsymbol{S} \boldsymbol{\varepsilon}
\end{gathered}
$$

where $\boldsymbol{h}$ contains the inertial forces that are quadratic with velocity.

To use the non-linear two-node superelement in flexible multibody models, the expressions in Eq. (2.40) can be included in the system level equations of motion. Note that in the assembly of elements, element interconnectivity can directly be realized by letting elements share the absolute nodal coordinates of the vector $\boldsymbol{x}$. An implementation of these expressions is realized in the SPACAR software [39].

\subsection{Numerical validation}

In this section we consider two examples to validate the non-linear two-node superelement by comparing simulation results with results obtained using non-linear beam elements. An additional example is included to test the performance of the non-linear two-node superelement for a more complex-shaped component. The first example is a planar spinning beam benchmark, from which results are also known in literature. The second example is a spinning beam attached to a universal joint, where full three-dimensional motion is considered. The last example is an unbalanced rotating shaft. This example demonstrates the effect of neglecting the small elastic deformations on the simulation accuracy for the different velocity transformation matrices. 


\subsubsection{Spinning beam}

The performance of the non-linear two-node superelement is demonstrated by analysing the spin-up motion of a planar flexible beam with uniform cross-section from [45], shown in Fig. 2.6(a). The aluminum beam has a length $L=8.0 \mathrm{~m}$ with a rectangular cross-section of $0.03675 \mathrm{~m}$ by $0.001986 \mathrm{~m}$. Its cross-sectional area $A$, moment of inertia $I$, density $\rho$ and Young's modulus $E$ are mentioned in the figure. The beam is accelerated from rest to a constant angular velocity. The prescribed spin-up motion is given by

$$
e(t)= \begin{cases}\frac{\Omega}{T}\left[\frac{1}{2} t^{2}-\frac{T^{2}}{4 \pi^{2}}\left(1-\cos \frac{2 \pi t}{T}\right)\right], & 0 \leq t \leq T \\ \Omega\left(t-\frac{1}{2} T\right), & t>T\end{cases}
$$

The final angular velocity is $\Omega=4 \mathrm{rad} / \mathrm{s}$ and the spin-up time is $T=15 \mathrm{~s}$, see Fig. 2.6(b).

To provide a reference for the superelement simulation results, the spinning beam is modelled using four non-linear planar beam elements whose elongation modes are suppressed, yielding a model with eight degrees of freedom and one prescribed coordinate. Similarly, four non-linear two-node superelements are used for the superelement models with their elongation modes suppressed. A single superelement is based on a linear FE model of a beam created using a large number of solid elements. The superelement models are referred to as Superelement $\boldsymbol{B}_{i}$ where $i$ denotes which velocity transformation matrix is used. Fig. 2.7 shows the tip deflection $\Delta y^{\prime}$ as a function of time for the superelement models and the model with non-linear beam elements. Only a small relative difference between the results of the non-linear beam and superelement models can be observed. Identical results were obtained for the superelement models with velocity transformation matrices $\boldsymbol{B}_{2}$ and

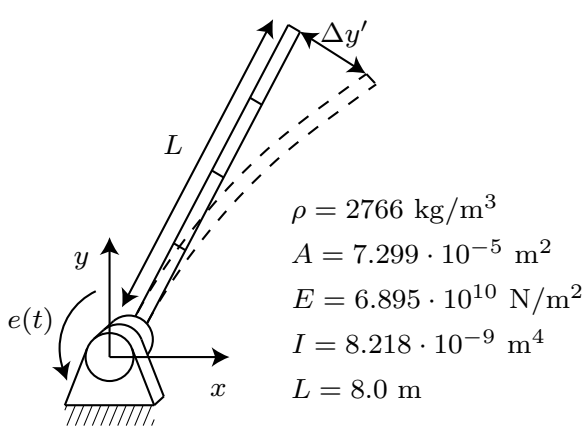

(a) Model parameters

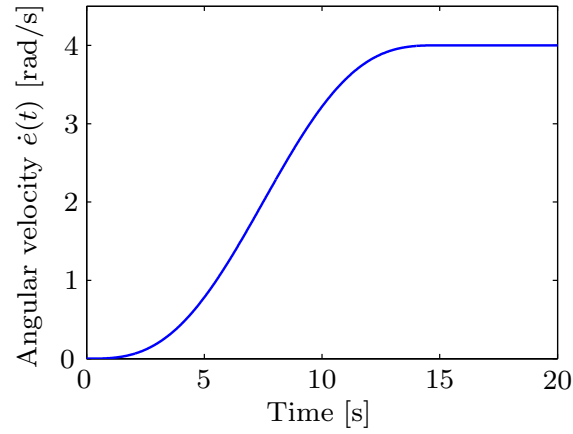

(b) Angular velocity profile

Figure 2.6: Spinning beam model parameters taken from [45] (a) and angular velocity profile (b). 


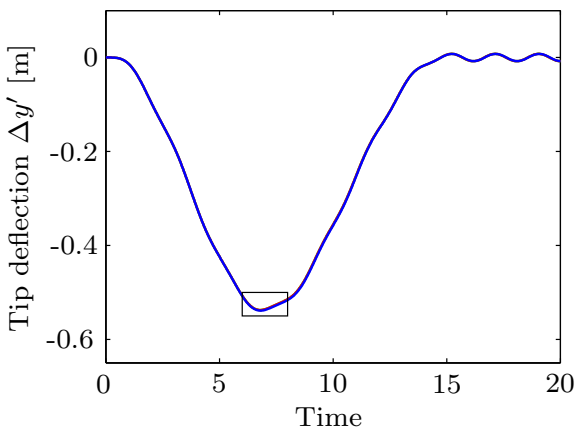

(a) Tip response

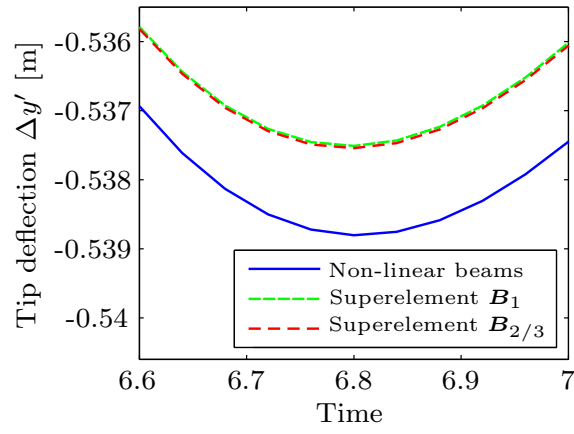

(b) Zoom of tip response

Figure 2.7: Tip response $\Delta y^{\prime}$ (a) and zoomed-in view (b) of the non-linear beam element and superelement models.

Table 2.1: Comparison of maximum tip deflections.

\begin{tabular}{lcc}
\hline Model & Number of elements & Max. deflection $[\mathrm{m}]$ \\
\hline SPACAR, non-linear beam & 4 & 0.5388 \\
SPACAR, superelement $\boldsymbol{B}_{1}$ & 4 superelements & 0.53751 \\
SPACAR, superelement $\boldsymbol{B}_{2 / 3}$ & 4 superelements & 0.53754 \\
Wu and Haug [71] & 4 substructures & 0.556 \\
idem & 6 substructures & 0.543
\end{tabular}

$\boldsymbol{B}_{3}$ from respectively Eq. (2.31) and Eq. (2.32). In fact, the velocity transformation matrices $\boldsymbol{B}_{2}$ and $\boldsymbol{B}_{3}$ will always give identical results for planar simulations, as the absolute angular velocity is in both cases expressed about the axis perpendicular to the plane of motion. Some small differences are observed for the simulation with velocity transformation matrix $\boldsymbol{B}_{1}$. Here the translational velocities are expressed in the node-attached reference frame, which differs slightly from the average orientation reference frame used by matrices $\boldsymbol{B}_{2}$ and $\boldsymbol{B}_{3}$.

In Tab. 2.1 the values of the maximum tip deflection, obtained by the non-linear beam element and superelement models, are compared with existing results. It can be concluded that they are quantitatively in good agreement.

\subsubsection{Spinning beam with out-of-plane bending}

In a second example we consider a spatial flexible beam which is connected by means of a universal joint to a point $O$ fixed in space, see Fig. 2.8. The beam has a length $L=8.0 \mathrm{~m}$ with a rectangular cross-section of $0.02 \mathrm{~m}$ by $0.04 \mathrm{~m}$. The material properties are the same as in the first example. The universal joint is modelled by two hinges with axes that initially coincide with the global $y$ - and $z$-axes. The relative 


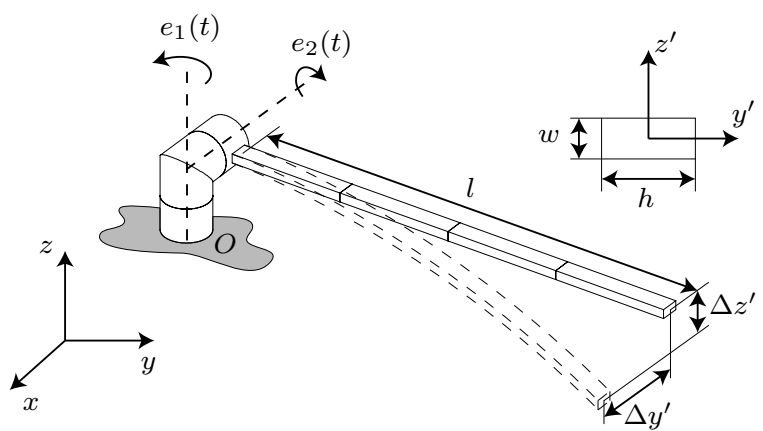

$\rho=2766 \mathrm{~kg} / \mathrm{m}^{3}$

$E=6.895 \cdot 10^{10} \mathrm{~N} / \mathrm{m}^{2}$

$h=0.04 \mathrm{~m}$

$w=0.02 \mathrm{~m}$

$l=8.0 \mathrm{~m}$

$\nu=0.3$

Figure 2.8: Spinning beam model parameters with out-of-plane excitation.

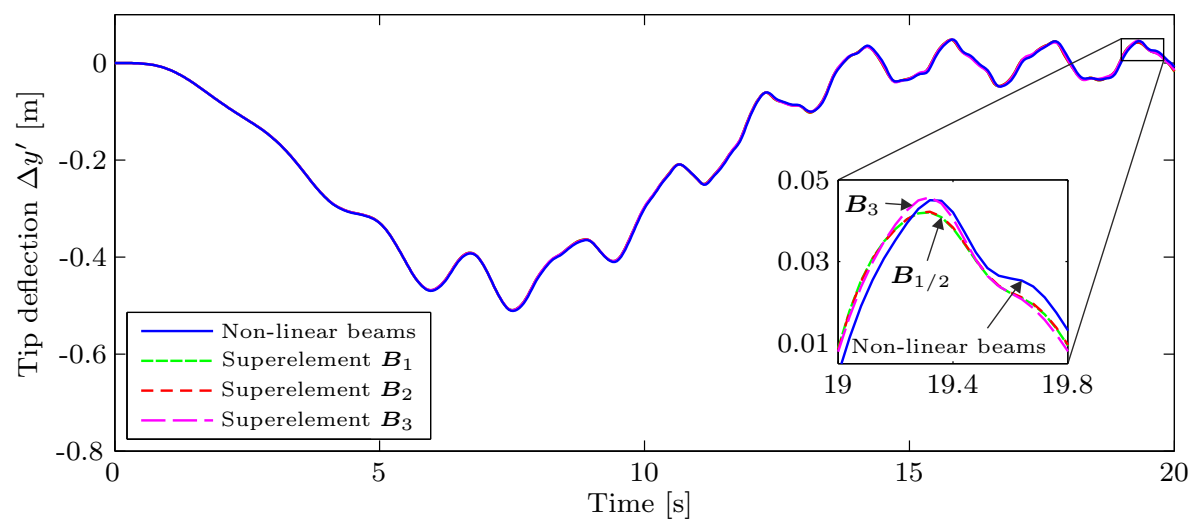

Figure 2.9: Tip deflection $\Delta y^{\prime}$ of the non-linear beam element and the superelement models.

rotation angles are denoted by $e_{1}$ and $e_{2}$, respectively, where $e_{1}(t)$ provides the spin-up motion described by Eq. (2.41) and $e_{2}(t)$ represents an additional excitation

$$
e_{2}(t)=0.01 \sin (15 t) \text {. }
$$

Similar to the previous example, four non-linear two-node superelements are used for the superelement models and their results are compared with those obtained with four non-linear beam elements. The elongation mode is suppressed for the superelement and the non-linear beam models, yielding models with 20 degrees of freedom and two prescribed coordinates.

Fig. 2.9 and Fig. 2.10 show the tip deflections $\Delta y^{\prime}$ and $\Delta z^{\prime}$ in the horizontal and vertical directions as is depicted in Fig. 2.8. It can be observed that the results of the superelement models are in good agreement with the non-linear beam element model. The superelement model with velocity transformation matrix $\boldsymbol{B}_{3}$ performs slightly 


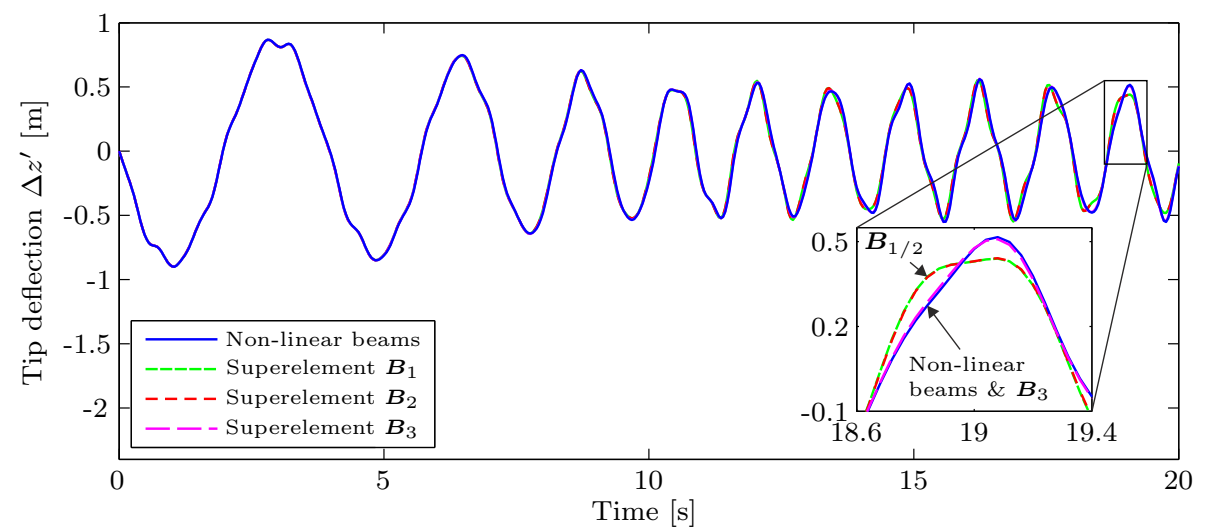

Figure 2.10: Tip deflection $\Delta z^{\prime}$ of the non-linear beam element and the superelement models.

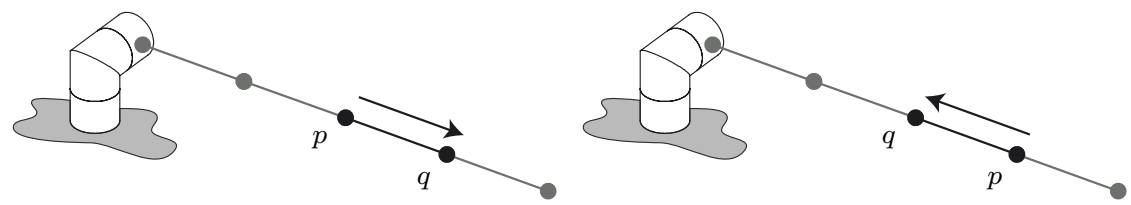

Figure 2.11: Direction of the elements in the model, original (left) and reversed (right).

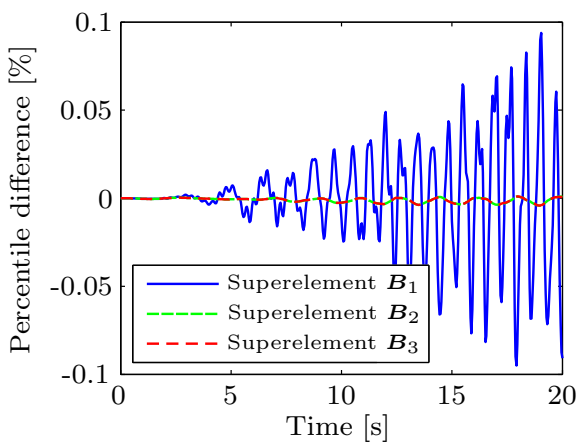

(a) Difference in $y^{\prime}$-direction

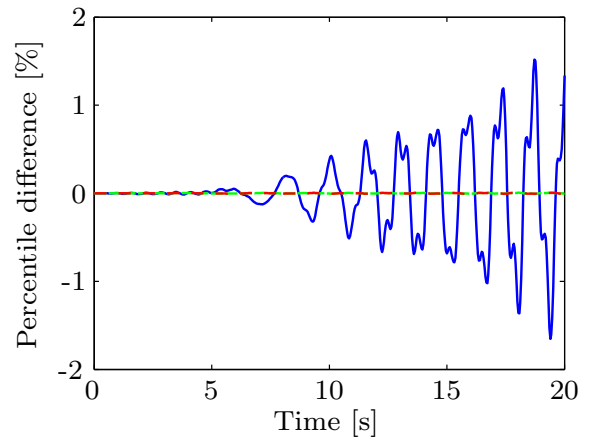

(b) Difference in $z^{\prime}$-direction

Figure 2.12: Comparison of the superelement models with the original direction of the elements and reversed. The differences in $y^{\prime}$-direction (a) are computed with respect to the maximal tip deflection $\Delta y_{\max }^{\prime}=0.5095 \mathrm{~m}$. The differences in $z^{\prime}$-direction (b) are computed with respect to the maximal tip deflection $\Delta z_{\text {max }}^{\prime}=0.8991 \mathrm{~m}$. 
better than the node attached and average reference frame approaches of matrices $\boldsymbol{B}_{1}$ and $\boldsymbol{B}_{2}$, respectively. A similar result is observed by Cardona [21].

For this example, we investigate the effect of reversing the direction of the elements on the simulation results, see Fig. 2.11. In Fig. 2.12 the differences are shown between the results of the original superelement models and with the direction of the elements reversed. It is clear that reversing the direction of the elements leads to a slightly different dynamical response for the node-attached reference frame approach of matrix $\boldsymbol{B}_{1}$. The small differences for the other two approaches can be attributed to numerical errors in the integration of the equations of motion. As these two approaches are less susceptible to the effect of reversing the direction of the elements, they can be considered to be better suited for the transformation of the nodal absolute velocities than the node-attached reference frame approach.

\subsubsection{Unbalanced rotating shaft}

For the third example, we investigate the effect of neglecting the small elastic deformations in Eq. (2.26) on the simulation accuracy for the different velocity transformation matrices. For this purpose we consider the unbalanced rotating shaft shown in Fig. 2.13. It consists of a cylindrical component eccentrically connected to two circular rods. The rods are connected to spherical joints which are supported by a normal support at one side and a rolling support at the other side. The spherical joints are modelled with three hinges in series whose axes are initially aligned with the global $x$-, $y$ - and $z$-axes. The shaft is accelerated around its axis from rest to a constant angular velocity. The prescribed spin-up motion, $e(t)$, is given by

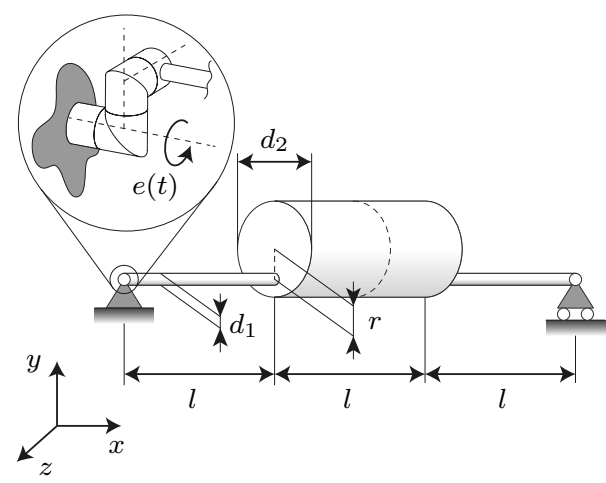

(a) Model Dimensions

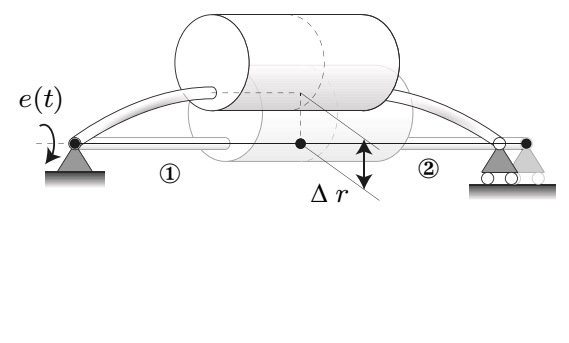

(b) Deflected state, modelled using two superelements (denoted by (1) and (2))

Figure 2.13: Model of an unbalanced rotating component attached to spherical joints at either end, supported by a rolling and a normal support. On the left, dimensional parameters are given (a) and on the right the deflected state due to rotation about the $x$-axis is shown (b). 
Table 2.2: Physical properties of the unbalanced rotating component.

\begin{tabular}{ll}
\hline Property & Value \\
\hline Diameter, $d_{1}$ & $2.0 \cdot 10^{-3} \mathrm{~m}$ \\
Diameter, $d_{2}$ & $3.0 \cdot 10^{-2} \mathrm{~m}$ \\
Length, $l$ & $7.5 \cdot 10^{-2} \mathrm{~m}$ \\
Eccentric offset, $r$ & $1.1 \cdot 10^{-2} \mathrm{~m}$ \\
Young's modulus, $E$ & $2.1 \cdot 10^{11} \mathrm{~N} / \mathrm{m}^{2}$ \\
Shear modulus, $G$ & $8.0 \cdot 10^{10} \mathrm{~N} / \mathrm{m}^{2}$ \\
Density, $\rho$ & $7800 \mathrm{~kg} / \mathrm{m}^{3}$
\end{tabular}

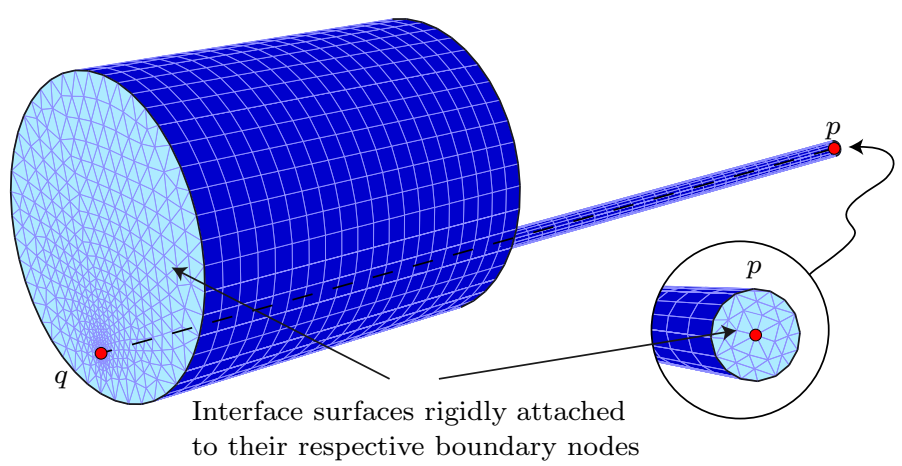

Figure 2.14: Superelement model depicting the $p$ and $q$ boundary nodes and their interface surfaces.

Eq. (2.41) with a final angular velocity of $\Omega=25 \mathrm{rad} / \mathrm{s}$ and a spin-up time of $T=15 \mathrm{~s}$. Furthermore, the physical properties of the shaft are given in Tab. 2.2.

Centrifugal forces, generated by the mass unbalance and the rotating motion, cause a deflection, $\Delta r$, as is shown in Fig. 2.13(b). As the deflection increases, the centrifugal forces will also increase, resulting in further deflection until an equilibrium is reached. Two superelements are used in the model, each representing half of the unbalanced rotating shaft. The boundary node locations are chosen such that the superelements are collinearly aligned, see Fig. 2.13(b). The nodes on the interface surfaces are rigidly connected to their respective $p$ and $q$ boundary nodes as is illustrated in Fig. 2.14. The FE model that is used as a basis for the superelement consists of approximately 36000 prism-shaped solid elements (ANsys Solid186 element).

For each of the velocity transformation matrices $\boldsymbol{B}_{1}, \boldsymbol{B}_{2}$ or $\boldsymbol{B}_{3}$, we will investigate the deflection $\Delta r$ as a function of time. As a reference, the final deflection is determined by a static analysis. In this analysis, a force $F_{c}(\Delta r)$ is applied at the middle node in the global $y$-direction which is equal to the centrifugal force at 
$T=15 \mathrm{~s}$. The centrifugal force is a function of the deflection $\Delta r$,

$$
F_{c}(\Delta r)=m_{u} \Omega^{2}(r+\Delta r)=0.4135 \cdot 25^{2}(0.011+\Delta r),
$$

where $m_{u}$ corresponds to the mass of the cylinder with diameter $d_{2}$ and $\Omega$ is the final angular velocity. The final deflection is estimated iteratively by increasing $\Delta r$ until an equilibrium is reached, resulting in a deflection of $\Delta r=1.44 \cdot 10^{-3} \mathrm{~m}$ corresponding to the horizontal line in Fig. 2.15(a) and Fig. 2.16(a).

First, we model the shaft by attaching the $q$-nodes of the two superelements, see Fig. 2.15(b). From the simulation results shown in Fig. 2.15(a), it can be observed that the choice of reference frame for transforming the absolute nodal velocities and estimating the kinetic energy has a large influence on the simulation results for this example. We note that even though the three velocity transformation matrices produce different results, the energy balance has been verified and remains zero within the simulation error tolerances for all three cases, indicating that the energy balance is in order. Yet correct simulation results are only obtained with the velocity transformation matrix $\boldsymbol{B}_{3}$, while the use of matrices $\boldsymbol{B}_{1}$ and $\boldsymbol{B}_{2}$ yields large steady state errors. The node-attached reference frame used in matrix $\boldsymbol{B}_{1}$ yields the largest steady state error. It is mentioned in Section 2.3.3 that for the node-attached reference frame, the error due to neglecting the small elastic deformations in the computation of the kinetic energy is introduced at node $q$. The observed error can then be explained by the fact that in this configuration most of the mass is concentrated at the $q$ nodes of the superelements. Then, by exchanging the location of the $p$ - and $q$-node, a significant smaller error should be realized. To test this, we model the shaft as is shown in Fig. 2.16(b) where the locations of the $p$ - and $q$-nodes

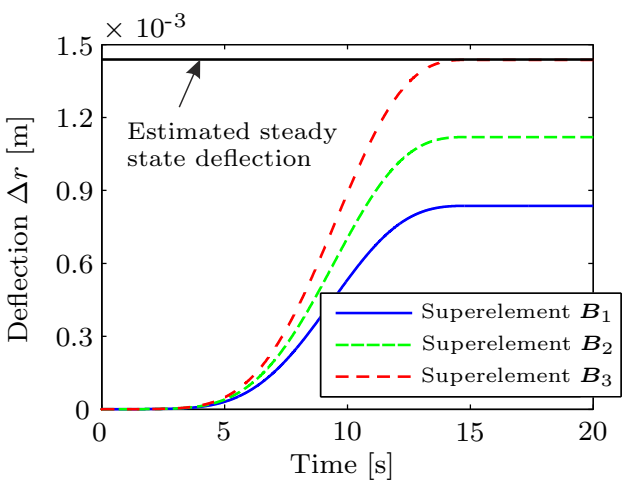

(a) Deflection $\Delta r$ as function of time

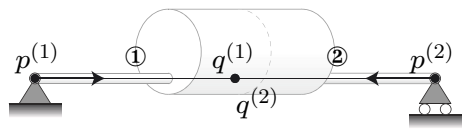

(b) Superelements defined with the $q$ nodes in the middle

Figure 2.15: The deflection due to centrifugal forces for the different velocity transformation matrices (a), when connecting the superelements by their $q$ nodes (b). 


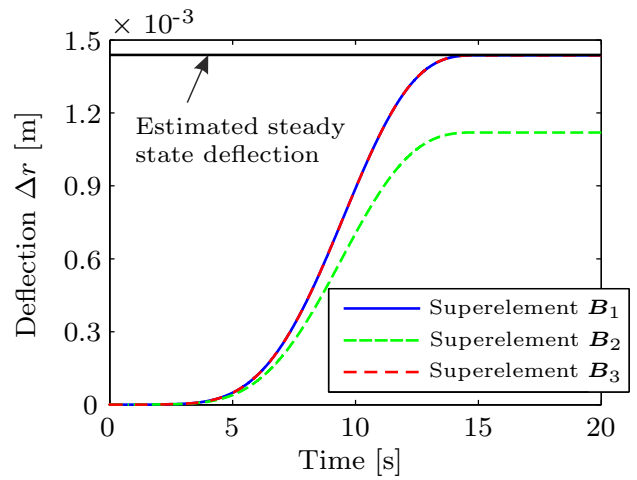

(a) Deflection $\Delta r$ as function of time

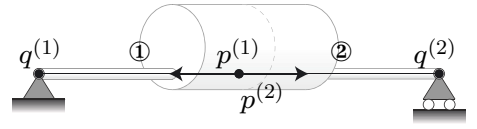

(b) Superelements defined with the $p$ nodes in the middle

Figure 2.16: The deflection due to centrifugal forces for the different velocity transformation matrices (a), when connecting the superelements by their $p$ nodes (b).

of the superelements are switched. From Fig. 2.16(a), it can be observed that correct results are indeed obtained in this configuration for the velocity transformation matrix $\boldsymbol{B}_{1}$. Exchanging the $p$ - and $q$-nodes has no effect on the results obtained with velocity transformation matrices $\boldsymbol{B}_{2}$ and $\boldsymbol{B}_{3}$. From these results we can conclude that the velocity transformation matrix $\boldsymbol{B}_{3}$ appears to be the most robust implementation considered for transforming the absolute nodal velocities.

\subsection{Conclusions}

In this paper a non-linear two-node superelement is proposed for the modelling of complex-shaped flexible members for use in multibody model simulations. In the approach, the kinematic model of a non-linear beam element is combined with a substructure method. This leads to a simple set of dynamic equations which can be easily implemented in flexible multibody software. The stiffness and mass matrix of the non-linear two-node superelement are based directly on the stiffness and mass matrix of a linear two-node superelement. This allows easy interfacing with existing FE software. Also, the exact rigid body solution is obtained if elastic deformations are not present.

Three approaches are considered for the transformation of the absolute nodal velocities. From numerical examples it can be observed that the first approach, a transformation with a node-attached reference frame, suffers from different dynamical responses if the direction of the elements in the model are reversed. The second approach, a transformation with an average orientation reference frame, is not susceptible to reversing the direction of the elements. In the third considered 
approach, the angular velocities are expressed in the reference frames attached to the superelement nodes, while the translational velocities are expressed in the average orientation reference frame. For the considered numerical examples, this approach yields the smallest errors and appears to be the most robust implementation for the transformation of the velocities.

In the present paper, only the constraint modes of the Craig-Bampton method are used in the non-linear two-node superelement. The constraint modes correctly preserve the static response to loads applied at the superelement nodes and are often sufficient for describing the first vibrational bending modes in a dynamic analysis as is demonstrated in the first and second example. Inclusion of the fixed-interface normal modes of the Craig-Bampton method will yield increased accuracy to dynamic loads and is considered in ongoing research.

Acknowledgements This research is financially supported by the Dutch association Point-One, project MOV-ET PNE08006, by the Dutch Department of Economic Affairs, Agriculture and Innovation. 


\title{
A non-linear two-node superelement with deformable-interface surfaces for use in flexible multibody systems
}

\begin{abstract}
A non-linear two-node superelement is proposed for efficient modelling of arbitrary-shaped flexible members with two interfaces in a flexible multibody model. The formulation is based on a small rotation and displacement hypothesis in a local co-rotational frame. Component mode substructuring methods can then be used to determine the dynamical properties of the superelement from a linear finite element model. The key contribution of this paper is the inclusion of so-called deformable-interface modes to model the deformability of the interface surfaces. This allows for an compliant connection to other superelements. With this capability, a component can be modelled with a number of superelements and its dynamical properties can be accurately analysed even for large deflections provided that the deformations remain small with respect to the co-rotational frame. Three examples demonstrate the applicability of the method. In the first example, large deflections of a relative short sheet flexure are analysed. Next, the formulation is used to obtain a dynamically reduced model of a complex-shaped component. In the third example, the time-response of a compliant mechanism is considered that is composed of the components of the first two examples. For all three examples, eigenfrequency results are in good agreement with results obtained using a classical non-linear finite element method.
\end{abstract}

\subsection{Introduction}

Non-linear flexible beam elements provide an effective approach to structural modelling of flexible multibody systems that consist of interconnected rigid and deformable components. A variety of well established non-linear beam element formalisms can be found in the literature $[28,43,62]$. The derivation of the beam

Submitted for publication in: Multibody System Dynamics (S.E. Boer, R.G.K.M. Aarts, J.P. Meijaard, D.M. Brouwer, J.B. Jonker) 
element is based on assumptions that limit its applicability to the modelling of slender components with uniform cross-section. However, in many applications, flexible members can be identified whose geometry does not comply with these assumptions. Examples are compliant cross-hinges with sheet flexures that have a large height to length ratio, i.e. short sheet flexures [68], or flexible components where the cross-sectional shape varies along the length [27]. For the modelling of mechanisms containing such components, it would be highly advantageous to have a beam-like element which is not restricted to the modelling of slender components with uniform cross-section.

In other words, there is a need for a so-called two-node superelement capable of modelling arbitrary-shaped flexible members with two interfaces whose numerical efficiency is close to that of a 'standard' beam element. An application for such superelements is the modelling of relatively short sheet flexures for which, in contrast to slender beams, correct modelling of the boundary effects is necessary. Particularly, the boundary effect due to a torsional load is of importance. From Saint-Venant's solution of the torsional problem of uniform beams, we know that a beam with noncircular cross-section will exhibit an out-of-plane deformation of the cross-section when twisted [66]. This so-called warping of the cross-section is described by the angle of twist per unit length and a warping function which depends on the cross-sectional shape. For short sheet flexures, the angle of twist per unit length will be relatively high compared with slender beams, resulting in significant warping of the cross-sections when twisted. If this warping is constrained due to clamping, the torsional stiffness will increase considerably. This effect is not predicted by classical beam theory. To account for this effect, several authors including Simo and Vu-Quoc [64], Gruttman et al. [30], Hsiao and Lin [35], Chen et al. [22] and Gonçalves et al. [29], modelled the warping of the cross-section with an extra degree of freedom which can be suppressed to model rigid clamping. It is however not trivial to determine the correct warping function for arbitrary cross-sectional shapes. Furthermore, the methods referred to in this paragraph are restricted to beams with a uniform cross-section and are not suited to describe components with varying cross-sectional shapes.

In a classical non-linear finite element (FE) analysis it is straightforward to analyse the dynamic behaviour of an arbitrary-shaped flexible member. However, it is in terms of computational time unavoidably costly to simulate mechanisms containing flexible components of arbitrary shape using such classical non-linear FE methods. Therefore, several authors including Shabana and Agrawal [4, 60], Yoo and Haug [72, 73] and Cardona and Géradin [19, 20, 21], adopted substructuring techniques in their multibody codes to allow efficient simulation of arbitrary-shaped components undergoing large rigid body motions and small elastic deformations. Such a substructure is obtained from projecting the dynamical properties of a linear FE model onto a lower-dimensional subspace spanned by so-called component modes. With substructuring methods it is possible to retain the physical meaning of nodes of the original FE model. These nodes can then be used to allow easy interfacing with other components. However, this can result in a large number of degrees of freedom of the substructure if all the nodes on the interface surface are retained. A common practice is to rigidly connect the nodes on the interface 
surfaces such that a single node can describe the positions and orientations of all the nodes on an interface surface.

In [12] (Chapter 2) this method is applied to realize a non-linear two-node superelement for the modelling of complex-shaped flexible beams undergoing large rigid body motions and small elastic deformations. Absolute nodal position and orientation coordinates are used to describe the configuration of the superelement in an inertial reference frame. For describing the elastic deformations independent of the rigid body motion, the concept of specifying independent deformation modes is adopted from a non-linear beam element description [38]. The deformation modes are defined in a co-rotational frame, which allows the use of linear stiffness models for the computation of the stress resultants if the elastic deformations remain small. The superelement mass and stiffness properties are determined from a linear FE model representing the complex-shaped component. The method yields analytically exact models for rigid components and provides accurate results when modelling flexible components exhibiting small elastic deformations. However, in either case, the two interface surfaces are considered to be rigid. This can cause artificial stiffening if the interface should instead behave compliant, e.g. for the modelling of torsional out-of-plane warping of the cross-section.

In this paper we extend the non-linear two-node superelement description in order to obtain a more accurate description for arbitrary-shaped flexible members and to account for deformations at the interface surfaces. This is accomplished by including so-called deformable-interface modes and fixed-interface normal modes from the Craig-Bamption method [23, 24]. The inclusion of the fixedinterface normal modes can increase the accuracy of the dynamic analysis. This is especially useful for the modelling of arbitrary-shaped components that only exhibit small deflections. With the addition of the deformable-interface modes, the deformability of the interface surfaces can be modelled. This is useful for the modelling of components that undergo large deflections where a single superelement is not sufficient to capture the geometrically non-linear behaviour. Then, the component should be discretized in a sufficient number of superelements such that the deformations of the individual superelements remain small allowing the use of linear stiffness models. Instead of having rigid interface surfaces between the superelements, they can be modelled deformable by using the deformable-interface modes. Although with this approach different elastic boundary effects can be modelled, we will specifically address the modelling of warping of the interface surfaces due to torsional rotations using these deformable-interface modes.

The contents of this paper are organized as follows. Following the approach described in [12], in Section 3.2, the derivation of a linear two-node superelement with deformable-interface modes and fixed-interface normal modes is described. Expressions are given for the potential and kinetic energy and the equivalent stiffness and mass matrices are derived. Then in Section 3.3, the derivation of the non-linear two-node superelement is presented and expressions are derived for its stiffness and mass matrices. Furthermore, by using Lagrange's equations, expressions for the stress resultants and the inertial forces are derived. In Section 3.4 it is explained how a proper connection between superelements with deformable- 
interface surfaces is realized. The approach is demonstrated in Section 3.5 using three examples inspired by a large-stroke compliant mechanism [27]. The first example will illustrate the use of deformable-interface modes for the modelling of large deflections of a short sheet flexure. In the second example the modelling of a component with varying cross-sectional geometry is considered. This example illustrates the use of fixed-interface normal modes for components exhibiting small deflections. In the third example, a mechanism is considered in which the components of the first two examples are combined and a time-response simulation is performed.

\subsection{Linear two-node superelement}

Component mode methods, such as the Craig-Bamption method [23, 24], can provide a reduced order description for the nodal displacements of a linear FE model by the use of component modes. In the first part of this section, we will introduce this description for the linear two-node superelement, as well as the concept of boundary nodes and interface surfaces. Then, we will explain the specific component modes considered for the linear two-node superelement. Subsequently, expressions are derived for the potential and kinetic energy, from which the reduced stiffness and mass matrix are obtained.

\subsubsection{Coordinates and components modes}

\section{Boundary nodes, interface surfaces and nodal displacements}

Figure 3.1 shows a linear FE model of a flexible complex-shaped component, which can be obtained by using FE software such as ANSYS ${ }^{\circledR}$. In order to construct a linear two-node superelement, boundary nodes $p$ and $q$ are introduced at either side

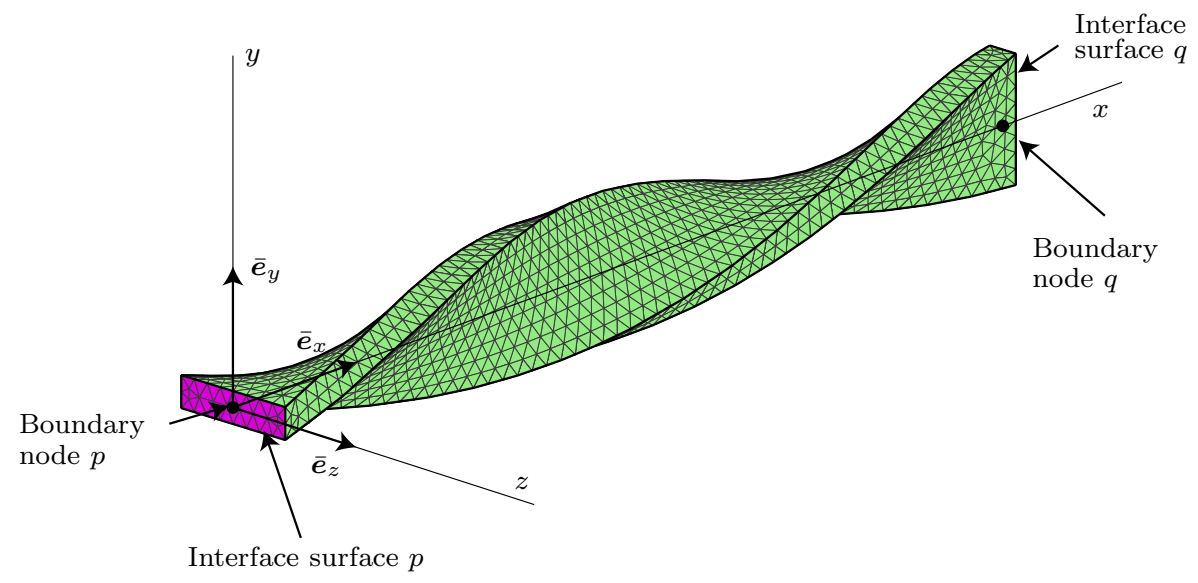

Figure 3.1: Finite element model of a flexible complex-shaped component. 
of the component. Furthermore, we introduce at these locations interface surfaces $p$ and $q$. The interface surfaces describe the region of contact to other components. In the initial undeformed configuration, the location of the coordinate system, with base vectors $\overline{\boldsymbol{e}}_{x}, \overline{\boldsymbol{e}}_{y}$ and $\overline{\boldsymbol{e}}_{z}$, is specified to coincide with node $p$ with its $x$-axis pointing towards node $q$. The orientation of the Y-axis can be chosen arbitrarily in the plane perpendicular to the $x$-axis, which defines the coordinate system $\left[\overline{\boldsymbol{e}}_{x}, \overline{\boldsymbol{e}}_{y}, \overline{\boldsymbol{e}}_{z}\right]$. Subsequently, an overbar denotes a kinematic quantity related to the coordinates system $\left[\overline{\boldsymbol{e}}_{x}, \overline{\boldsymbol{e}}_{y}, \overline{\boldsymbol{e}}_{z}\right]$.

By using the concept of component modes such as is defined in the Craig-Bampton method, we can write the nodal displacements $\overline{\boldsymbol{u}}$ of the linear FE model in this coordinate system as

$$
\overline{\boldsymbol{u}}=\boldsymbol{V} \overline{\boldsymbol{\mu}}+\boldsymbol{W} \overline{\boldsymbol{\eta}} .
$$

Here the matrix $\boldsymbol{V}$ represents the so-called constraint modes of the Craig-Bampton method with associated boundary coordinates $\overline{\boldsymbol{\mu}}$. Furthermore, the matrix $\boldsymbol{W}$ can contain other types of component modes with associated generalized coordinates $\overline{\boldsymbol{\eta}}$. Specifically, for the linear two-node superelement we write for these modes

$$
\boldsymbol{W}=\left[\boldsymbol{W}_{d}, \boldsymbol{W}_{f}\right],
$$

where we refer to $\boldsymbol{W}_{d}$ as deformable-interface modes describing the deformability of the interface surfaces and $\boldsymbol{W}_{f}$ represents the fixed-interface normal modes of the Craig-Bampton method describing the internal flexibility of the component with fixed interface surfaces. In the subsequent sections the constraint modes, deformable-interface modes and the fixed-interface normal modes are explained in detail.

\section{Constraint modes}

Finite element models of complex-shaped components are commonly created using solid elements which only have translational degrees of freedom. To be able to apply both forces and moments to a single boundary node, both translational and rotational coordinates are required in the superelement formalism. To achieve this for a FE model with only translational degrees of freedom, the nodes on the interface surfaces can be considered to be rigidly attached to the boundary nodes. The displacement and orientation of the boundary nodes then prescribe the displacement and orientation of the interface surfaces. The boundary node displacements and rotations are collected in the vector of boundary coordinates $\overline{\boldsymbol{\mu}}$,

$$
\overline{\boldsymbol{\mu}}=\left\{\begin{array}{llll}
\overline{\boldsymbol{u}}^{p \mathrm{~T}}, & \overline{\boldsymbol{\varphi}}^{p \mathrm{~T}}, & \overline{\boldsymbol{u}}^{q \mathrm{~T}}, & \bar{\varphi}^{q \mathrm{~T}}
\end{array}\right\}^{\mathrm{T}},
$$

where $\overline{\boldsymbol{u}}^{p}, \overline{\boldsymbol{\varphi}}^{p}, \overline{\boldsymbol{u}}^{q}$ and $\overline{\boldsymbol{\varphi}}^{q}$ are the nodal displacement and rotation vectors of boundary nodes $p$ and $q$ respectively. This set of coordinates are related to the constraint modes of the Craig-Bampton method. A constraint mode is defined as the static deformation of a component generated by applying a unit displacement on a boundary coordinate while fixing all other boundary coordinates [24]. The resulting set of constraint modes spans the static response of the component to loads applied on the boundary nodes. 

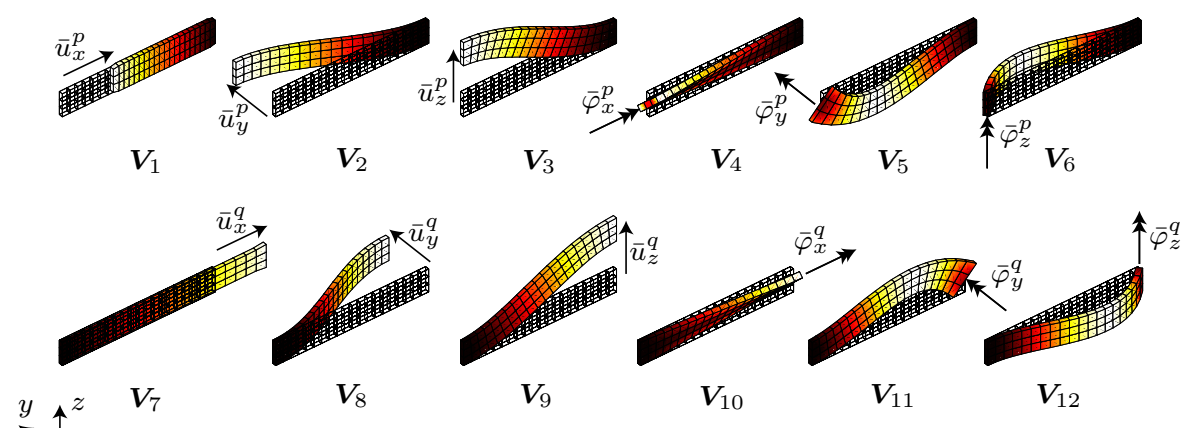

$\coprod^{y} \uparrow_{x}^{z}$

Figure 3.2: The twelve constraint modes of a FE model of a beam, as they appear column-wise in the matrix $\boldsymbol{V}$ with associated boundary coordinates.

The two-node superelement has a total of twelve constraint modes, three rotational and three translational modes at either side, see Fig. 3.2.

\section{Deformable-interface modes}

To account for deformations at the interface surfaces, additional flexibility can be added to the superelement by considering so-called deformable-interface modes. This class of modes encompasses any type of mode that describes the deformation of the interface surfaces.

In particular, we will focus on a type of deformable-interface mode referred to as a warping mode. The warping modes are for example of importance for the correct modelling of relatively short compliant sheet flexures and tubes with open crosssections. We define a warping mode by the out-of-plane warping deformations of an interface surface due to twist of a flexible component, while the other interface surface is fixed. Two warping modes can be computed for a superelement, i.e. one warping mode on each side. From Saint-Venant's solution of the torsional problem of uniform beams with non-circular cross-section, we know that the deformations of a twisted flexible component may be considered as a rotation of the cross-section and an out-of-plane warping of the cross-section [66]. Therefore, to compute a warping mode for a non-uniform component, we assume that the nodal displacements at the interface surface due to twist consists of a rotational part and an out-of-plane warping part. The warping mode corresponds to the deformations of the out-of-plane warping part. These deformations can be computed by determining the nodal displacement differences between a FE analysis where an interface surface is rigidly rotated in the torsional direction and a FE analysis where, during this torsional rotation, the interface surface remains free to deform in the out-of-plane direction.

As an example for the computation of a warping mode we consider the FE model shown in Fig. 3.3(a). Here we compute a warping mode at the $q$-side of the component while fixing the $p$-side. The interface surface and the surface unit normal, $\overline{\boldsymbol{n}}^{q}$, are defined at the $q$-side of the component. The torsion mode with the rigid interface 


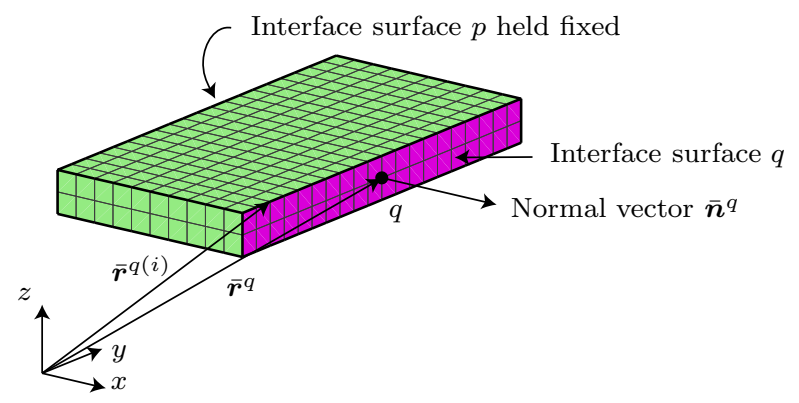

(a) Linear FE model

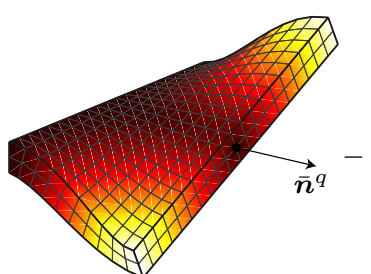

Torsion mode with rigid interface surface

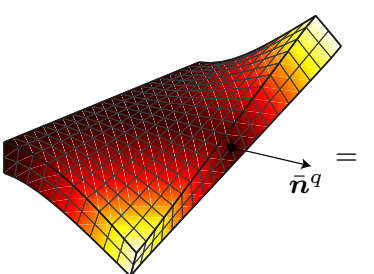

Torsion mode where the interface surface remains free to deform in the out-of-plane direction

(b) Warping mode

surface shown in Fig. 3.3(b) can be computed by prescribing the nodal displacements $\overline{\boldsymbol{u}}^{q(i)}$ of the $i^{\text {th }}$ node of interface surface $q$ according to

$$
\overline{\boldsymbol{u}}^{q(i)}=\overline{\boldsymbol{n}}^{q} \times\left(\overline{\boldsymbol{r}}^{q(i)}-\overline{\boldsymbol{r}}^{q}\right),
$$

where $\overline{\boldsymbol{r}}^{q}$ and $\overline{\boldsymbol{r}}^{q(i)}$ are respectively the position vectors of boundary node $q$ and the $i^{\text {th }}$ node of interface surface $q$, see Fig. 3.3(a). For the computation of the other torsion mode of Fig. 3.3(b), the same nodal displacements as in Eq. (3.4) are used with the modification that nodal displacements in the $\overline{\boldsymbol{n}}^{q}$ direction are not prescribed.

The warping mode $\boldsymbol{W}_{d}^{q}$ is then obtained by determining the difference between the two torsion modes. In a similar manner the $p$-side warping mode $\boldsymbol{W}_{d}^{p}$ is computed while fixing the $q$-side. Both warping modes are collected in the matrix $\boldsymbol{W}_{d}$ of Eq. (3.2),

$$
\boldsymbol{W}_{d}=\left[\boldsymbol{W}_{d}^{p}, \boldsymbol{W}_{d}^{q}\right]
$$




\section{Fixed-interface normal modes}

With the inclusion of the fixed-interface normal modes, the dynamical model of the linear two-node superelement is refined. This is especially beneficial when the constraint modes are not sufficient for describing the vibrational modes of interest of the component. The normal modes can be obtained from the solution of the generalized eigenvalue problem with the interface surfaces held fixed, see [24]. To reduce the number of modal coordinates, a truncated set of eigenvectors is used and stored in the matrix $\boldsymbol{W}_{f}$ of Eq. (3.2).

\subsubsection{Potential energy}

With Eq. (3.1), the potential energy of the linear two-node superelement can be expressed in terms of the reduced set of coordinates $\overline{\boldsymbol{\mu}}$ and $\overline{\boldsymbol{\eta}}$. Note that the constraint modes implicitly contain all six rigid body modes. For example, $\boldsymbol{V}_{1}+\boldsymbol{V}_{7}$ results in a rigid body translation in the $x$-direction. With similar linear combinations of the constraint modes, all rigid body movements can be described. Obviously, the rigid body modes do not contribute to the elastic potential energy of the component and only the contributions of the elastic deformations are of interest. A 6-D subspace of the constraint mode matrix $\boldsymbol{V}$ spans the space associated with purely elastic deformations. The choice of base vectors for this subspace is not unique. A possible choice consists of the constraint modes belonging to the elongation $\boldsymbol{V}_{7}$, torsion $\boldsymbol{V}_{10}$, and the four bending modes $\boldsymbol{V}_{5}, \boldsymbol{V}_{11}$ and $\boldsymbol{V}_{6}, \boldsymbol{V}_{12}$. The vector of elastic nodal displacements, $\overline{\boldsymbol{u}}_{\mathrm{e}}$, of the linear FE model can then be expressed in terms of this subset of constraint modes and the matrix $\boldsymbol{W}$ containing the deformable and fixed-interface modes,

$$
\overline{\boldsymbol{u}}_{\mathrm{e}}=\left[\boldsymbol{V}_{\mathrm{e}} \boldsymbol{W}\right]\left\{\begin{array}{c}
\overline{\boldsymbol{\mu}}_{\mathrm{e}} \\
\overline{\boldsymbol{\eta}}
\end{array}\right\}
$$

where

and

$$
\boldsymbol{V}_{\mathrm{e}}=\left[\begin{array}{llllll}
\boldsymbol{V}_{7}, & \boldsymbol{V}_{10}, & \boldsymbol{V}_{5}, & \boldsymbol{V}_{11}, & \boldsymbol{V}_{6}, & \boldsymbol{V}_{12}
\end{array}\right]
$$

$$
\overline{\boldsymbol{\mu}}_{\mathrm{e}}=\left\{\begin{array}{ccccc}
\bar{u}_{x}^{q}, & \bar{\varphi}_{x}^{q}, \quad \bar{\varphi}_{y}^{p}, \quad \bar{\varphi}_{y}^{q}, \quad \bar{\varphi}_{z}^{p}, \quad \bar{\varphi}_{z}^{q}
\end{array}\right\}^{\mathrm{T}} .
$$

Here $\boldsymbol{V}_{\mathrm{e}}$ is the matrix containing the deformation modes with associated deformation boundary coordinates $\overline{\boldsymbol{\mu}}_{\mathrm{e}}$.

With Eq. (3.6a) the potential energy $P$ of the superelement can be expressed in terms of the coordinate vectors $\overline{\boldsymbol{\mu}}_{\mathrm{e}}$ and $\overline{\boldsymbol{\eta}}$,

$$
P=\frac{1}{2} \overline{\boldsymbol{u}}_{\mathrm{e}}^{\mathrm{T}} \overline{\boldsymbol{K}}_{\mathrm{FEM}} \overline{\boldsymbol{u}}_{\mathrm{e}}=\frac{1}{2}\left\{\overline{\boldsymbol{\mu}}_{\mathrm{e}}^{\mathrm{T}} \overline{\boldsymbol{\eta}}^{\mathrm{T}}\right\}\left[\begin{array}{c}
\boldsymbol{V}_{\mathrm{e}}^{\mathrm{T}} \\
\boldsymbol{W}^{\mathrm{T}}
\end{array}\right] \overline{\boldsymbol{K}}_{\mathrm{FEM}}\left[\boldsymbol{V}_{\mathrm{e}} \boldsymbol{W}\right]\left\{\begin{array}{c}
\overline{\boldsymbol{\mu}}_{\mathrm{e}} \\
\overline{\boldsymbol{\eta}}
\end{array}\right\} .
$$

where $\overline{\boldsymbol{K}}_{\mathrm{FEM}}$ is the stiffness matrix of the linear FE model. From Eq. (3.7), we can define the reduced stiffness matrix $\bar{S}$ as

$$
\overline{\boldsymbol{S}}=\left[\begin{array}{c}
\boldsymbol{V}_{\mathrm{e}}^{\mathrm{T}} \\
\boldsymbol{W}^{\mathrm{T}}
\end{array}\right] \overline{\boldsymbol{K}}_{\mathrm{FEM}}\left[\boldsymbol{V}_{\mathrm{e}} \boldsymbol{W}\right]
$$

It should be noted that the modes collected in the matrix $\left[\boldsymbol{V}_{\mathrm{e}} \boldsymbol{W}\right]$ have to be linearly independent to obtain a stiffness matrix $\overline{\boldsymbol{S}}$ of full rank. 


\subsubsection{Kinetic energy}

To determine the expression for the kinetic energy, Eq. (3.1) is differentiated with respect to time, yielding

$$
\dot{\overline{\boldsymbol{u}}}=[\boldsymbol{V} \boldsymbol{W}]\left\{\begin{array}{c}
\overline{\boldsymbol{v}} \\
\dot{\overline{\boldsymbol{\eta}}}
\end{array}\right\} \quad \text { with } \quad \overline{\boldsymbol{v}}=\dot{\overline{\boldsymbol{\mu}}}
$$

and

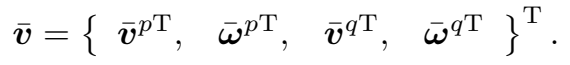

Here $\overline{\boldsymbol{v}}$ is the vector of boundary velocities containing the boundary node translational velocities $\overline{\boldsymbol{v}}^{p}$ and $\overline{\boldsymbol{v}}^{q}$, and angular velocities $\overline{\boldsymbol{\omega}}^{p}$ and $\overline{\boldsymbol{\omega}}^{q}$, of nodes $p$ and $q$ respectively. Their components are expressed along the base vectors $\overline{\boldsymbol{e}}_{x}, \overline{\boldsymbol{e}}_{y}$ and $\overline{\boldsymbol{e}}_{z}$. Furthermore, $\dot{\overline{\boldsymbol{\eta}}}$ are the time derivatives of the vector $\overline{\boldsymbol{\eta}}$ from Eq. (3.1). Using Eq. (3.9a) the kinetic energy $T$ can be expressed as

$$
T=\frac{1}{2} \dot{\overline{\boldsymbol{u}}}^{\mathrm{T}} \overline{\boldsymbol{M}}_{\mathrm{FEM}} \dot{\overline{\boldsymbol{u}}}=\frac{1}{2}\left\{\overline{\boldsymbol{v}}^{\mathrm{T}} \dot{\overline{\boldsymbol{\eta}}}^{\mathrm{T}}\right\}\left[\begin{array}{c}
\boldsymbol{V}^{\mathrm{T}} \\
\boldsymbol{W}^{\mathrm{T}}
\end{array}\right] \overline{\boldsymbol{M}}_{\mathrm{FEM}}[\boldsymbol{V} \quad \boldsymbol{W}]\left\{\begin{array}{c}
\overline{\boldsymbol{v}} \\
\dot{\overline{\boldsymbol{\eta}}}
\end{array}\right\} .
$$

where $\overline{\boldsymbol{M}}_{\mathrm{FEM}}$ is the mass matrix of the linear FE model. From Eq. (3.10), we can define the reduced mass matrix $\bar{M}$ to be

$$
\overline{\boldsymbol{M}}=\left[\begin{array}{c}
\boldsymbol{V}^{\mathrm{T}} \\
\boldsymbol{W}^{\mathrm{T}}
\end{array}\right] \overline{\boldsymbol{M}}_{\mathrm{FEM}}[\boldsymbol{V} \boldsymbol{W}] .
$$

Here, the modes collected in the matrix $[\boldsymbol{V} \boldsymbol{W}]$ have to be linearly independent to obtain a mass matrix $\bar{M}$ of full rank.

\subsection{Non-linear two-node superelement}

In order to develop a non-linear two-node superelement capable of describing large rigid body rotations and small deformations, a kinematic model is required that correctly describes the element's configuration in 3-D space when undergoing large rigid body rotations. The first part of this section describes the kinematic model that is used for this purpose. Then in the second and third part, using the expressions for the potential and kinetic energy of the linear two-node superelement, we derive expressions for the potential and kinetic energy of the non-linear two-node superelement. Its dynamic relations can then be derived with the aid of Lagrange's equations.

\subsubsection{Coordinates and modes}

The definition of the nodal coordinates of the non-linear superelement and the specification of a related set of deformation coordinates are in accordance with the non-linear beam element introduced in [38]. The global configuration of the non-linear superelement is defined by the position vectors $\boldsymbol{r}^{p}$ and $\boldsymbol{r}^{q}$ and the 


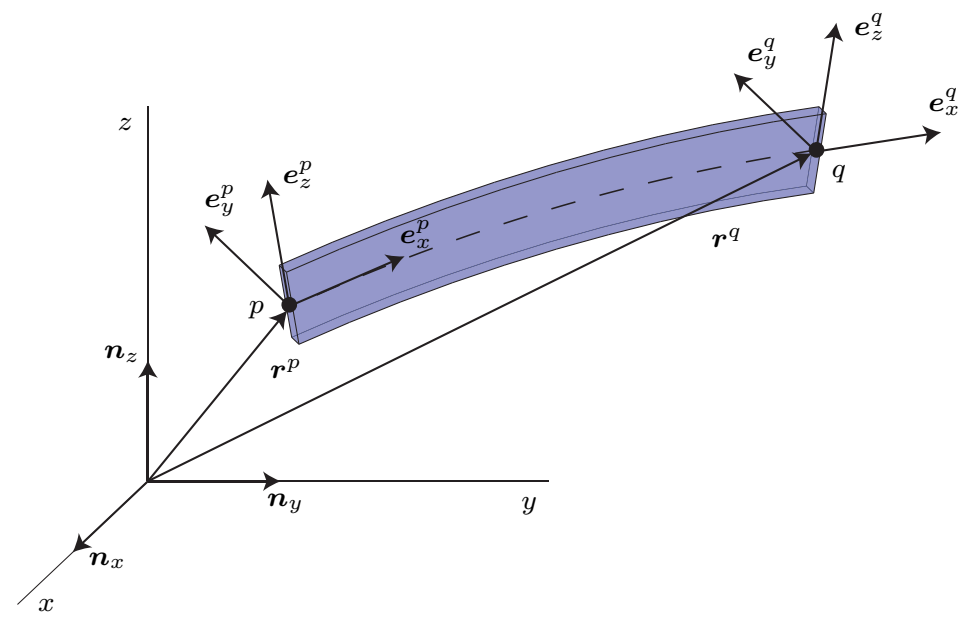

Figure 3.4: The global configuration of the non-linear two-node superelement expressed in the inertial reference frame.

orientation of the orthonormal triads $\left[\boldsymbol{e}_{x}^{p}, \boldsymbol{e}_{y}^{p}, \boldsymbol{e}_{z}^{p}\right]$ and $\left[\boldsymbol{e}_{x}^{q}, \boldsymbol{e}_{y}^{q}, \boldsymbol{e}_{z}^{q}\right]$ rigidly attached to the interface surfaces at nodes $p$ and $q$, see Fig. 3.4. In the initial undeflected state, the triads coincide with the initial orientation described by the rotation matrix $\boldsymbol{R}_{0}$. In a deflected state, the orientation of the triads can be computed from the rotation matrices $\boldsymbol{R}^{p}$ and $\boldsymbol{R}^{q}$ which describe the orientation of nodes $p$ and $q$ with respect to the initial orientation,

$$
\begin{aligned}
{\left[\begin{array}{lll}
\boldsymbol{e}_{x}^{p}, & \boldsymbol{e}_{y}^{p}, & \boldsymbol{e}_{z}^{p}
\end{array}\right] } & =\boldsymbol{R}^{p} \boldsymbol{R}_{0}\left[\begin{array}{lll}
\boldsymbol{n}_{x}, & \boldsymbol{n}_{y}, & \boldsymbol{n}_{z}
\end{array}\right], \\
{\left[\begin{array}{lll}
\boldsymbol{e}_{x}^{q}, & \boldsymbol{e}_{y}^{q}, & \boldsymbol{e}_{z}^{q}
\end{array}\right] } & =\boldsymbol{R}^{q} \boldsymbol{R}_{0}\left[\begin{array}{lll}
\boldsymbol{n}_{x}, & \boldsymbol{n}_{y}, & \boldsymbol{n}_{z}
\end{array}\right] .
\end{aligned}
$$

Here $\boldsymbol{n}_{x}, \boldsymbol{n}_{y}$ and $\boldsymbol{n}_{z}$ are the unit vectors of the inertial reference frame. The initial orientation $\boldsymbol{R}_{0}$ is used such that in the assembly of the finite elements the rotation coordinates that parametrize rotation matrices $\boldsymbol{R}^{p}$ and $\boldsymbol{R}^{q}$ can be shared by multiple elements. The parametrization of rotations can be accomplished in several ways, such as by modified Euler angles, Euler parameters, Rodrigues parameters or the Cartesian rotation vector [28]. In this paper we use Euler parameters for the parametrization of rotations to avoid singularity problems associated with Euler angles and to avoid the use of trigonometric functions. Instead, the components of a rotation matrix $\boldsymbol{R}$, parametrized with Euler parameters, are computationally efficient quadratic algebraic expressions [38].

With this parametrization the nodal coordinates of the non-linear two-node superelement are two sets of Cartesian coordinates $\boldsymbol{r}^{p}$ and $\boldsymbol{r}^{q}$, describing the nodal positions in the inertial coordinate system and two sets of Euler parameters, $\boldsymbol{\lambda}^{p}$ and $\boldsymbol{\lambda}^{q}$, representing the orientation of the triads $\left[\boldsymbol{e}_{x}^{p}, \boldsymbol{e}_{y}^{p}, \boldsymbol{e}_{z}^{p}\right]$ and $\left[\boldsymbol{e}_{x}^{q}, \boldsymbol{e}_{y}^{q}, \boldsymbol{e}_{z}^{q}\right]$ at 
nodes $p$ and $q$. The vector $\boldsymbol{x}$ of nodal coordinates can then be written as

$$
\boldsymbol{x}=\left\{\begin{array}{llll}
\boldsymbol{r}^{p \mathrm{~T}}, & \boldsymbol{\lambda}^{p \mathrm{~T}}, & \boldsymbol{r}^{q \mathrm{~T}}, & \boldsymbol{\lambda}^{q \mathrm{~T}}
\end{array}\right\}^{\mathrm{T}} .
$$

Note that the parametrization of rotations is redundant due to the constraint $\boldsymbol{\lambda}^{\mathrm{T}} \boldsymbol{\lambda}=$ 1, imposed on the Euler parameters of Eq. (3.13). This results in a total of twelve independent nodal coordinates.

The configuration defined by the nodal coordinate vector $\boldsymbol{x}$ also includes the rigid body orientation and position of the superelement. These rigid body modes should be removed to determine the elastic deformations. To achieve this we employ the concept of specifying independent deformation modes from the non-linear beam element model $[38,43]$. As the superelement has twelve independent nodal coordinates and six rigid body modes, we can define six independent deformation modes. The set of associated deformation coordinates $\varepsilon$ can be expressed as analytical functions of the nodal coordinate vector $\boldsymbol{x}$ and the reference length $l_{0}$ [43],

where

$$
\varepsilon=\mathcal{D}(\boldsymbol{x}),
$$

$$
\begin{aligned}
& \varepsilon_{1}=l-l_{0}, \\
& \varepsilon_{2}=l_{0}\left(\boldsymbol{e}_{z}^{p} \cdot \boldsymbol{e}_{y}^{q}-\boldsymbol{e}_{y}^{p} \cdot \boldsymbol{e}_{z}^{q}\right) / 2, \\
& \varepsilon_{3}=-l_{0} \boldsymbol{e}_{l} \cdot \boldsymbol{e}_{z}^{p}, \\
& \varepsilon_{4}=l_{0} \boldsymbol{e}_{l} \cdot \boldsymbol{e}_{z}^{q}, \\
& \varepsilon_{5}=l_{0} \boldsymbol{e}_{l} \cdot \boldsymbol{e}_{y}^{p}, \\
& \varepsilon_{6}=-l_{0} \boldsymbol{e}_{l} \cdot \boldsymbol{e}_{y}^{q},
\end{aligned}
$$

with

$$
l=\left\|\boldsymbol{r}^{q}-\boldsymbol{r}^{p}\right\| \quad \text { and } \quad \boldsymbol{e}_{l}=\left(\boldsymbol{r}^{q}-\boldsymbol{r}^{p}\right) / l .
$$

Here $\boldsymbol{e}_{y}^{p}, \boldsymbol{e}_{z}^{p}, \boldsymbol{e}_{y}^{q}$ and $\boldsymbol{e}_{z}^{q}$ are defined by Eq. (3.12). The deformation coordinates represent a set of deformation modes, where the first deformation coordinate, $\varepsilon_{1}$, describes the elongation of the element, the second coordinate, $\varepsilon_{2}$, describes the torsion, and the remaining coordinates are associated with the bending deformations. The deformation modes are visualized in Fig. 3.5. The definitions of the deformation coordinates are invariant under arbitrary rigid body motion of the superelement. We note that the method outlined here is not particularly restricted to the set of deformation coordinates given above. Other descriptions that describe six independent deformation modes in terms of the absolute nodal coordinates could be used as well.

These six deformation modes are however not adequate for modelling the deformation of the interface surfaces and may not be sufficient for modelling the vibrational modes of interest of a component. By reintroducing the coordinate system $\left[\overline{\boldsymbol{e}}_{x}, \overline{\boldsymbol{e}}_{y}, \overline{\boldsymbol{e}}_{z}\right]$ from the linear superelement in Fig. 3.1 as the co-rotational frame of the non-linear superelement, we can define an additional set of generalized coordinates $\bar{\eta}$ that are independent of the deformation coordinates $\varepsilon$,

$$
\overline{\boldsymbol{\eta}}=\left\{\begin{array}{lll}
\overline{\boldsymbol{\eta}}_{d}^{p \mathrm{~T}}, & \overline{\boldsymbol{\eta}}_{d}^{q \mathrm{~T}}, & \overline{\boldsymbol{\eta}}_{f}^{\mathrm{T}}
\end{array}\right\}^{\mathrm{T}},
$$



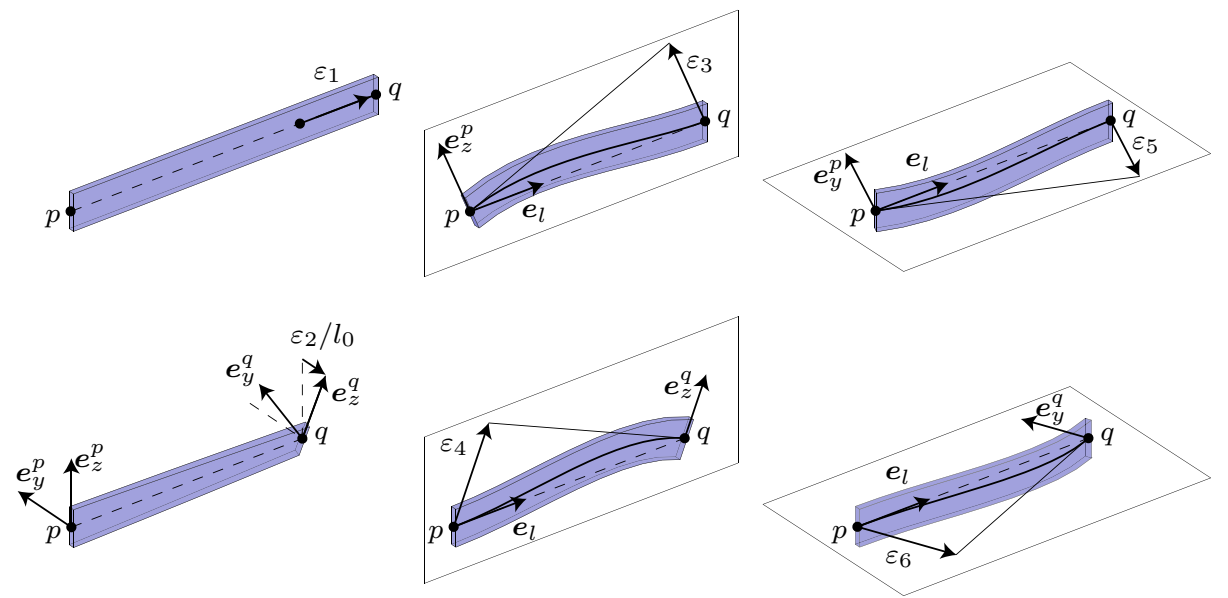

Figure 3.5: Graphical representation of the deformation modes with deformation coordinates $\varepsilon_{1}$ through $\varepsilon_{6}$.

where $\overline{\boldsymbol{\eta}}_{d}^{p}$ and $\overline{\boldsymbol{\eta}}_{d}^{q}$ are the coordinates associated with the deformable-interface modes of Section 3.2.1 at respectively the $p$ and $q$-side and $\overline{\boldsymbol{\eta}}_{f}$ are the coordinates associated with the fixed-interface normal modes discussed in Section 3.2.1.

To summarize, the configuration of the non-linear two-node superelement is defined by the nodal coordinates $\boldsymbol{x}$ and the generalized coordinates $\overline{\boldsymbol{\eta}}$ of Eqs. (3.13) and (3.15), respectively. Furthermore, the elastic deformations are described by the deformation modes with associated deformation coordinates $\varepsilon$ of Eq. (3.14) and the deformable-interface and fixed-interface normal modes with associated generalized coordinates $\bar{\eta}$.

\subsubsection{Potential energy}

\section{Relating deformation coordinates}

With an expression for the potential energy, the internal forces acting on the superelement can be determined. The potential energy can be expressed in terms of the deformation coordinates $\boldsymbol{\varepsilon}$ and the set of generalized coordinates $\overline{\boldsymbol{\eta}}$ of Eqs. (3.14) and (3.15) respectively,

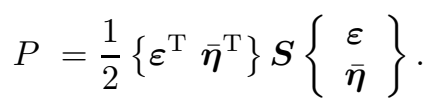

Here $\boldsymbol{S}$ is the stiffness matrix of the non-linear two-node superelement.

An expression for $\boldsymbol{S}$ can be obtained by relating Eq. (3.16) with the potential energy of the linear superelement given by Eq. (3.7). Note that the generalized coordinates $\overline{\boldsymbol{\eta}}$ of Eq. (3.15) are identical to those of Eq. (3.7). Then, by determining the relation between the deformation coordinates $\varepsilon$ and the deformation boundary 
coordinates $\overline{\boldsymbol{\mu}}_{\mathrm{e}}$, an expression for $\boldsymbol{S}$ can be derived. For infinitesimal elastic deformations the deformation coordinates $\varepsilon$ of Eq. (3.14b) may be approximated by the linearized deformations with respect to the co-rotational frame $\left[\overline{\boldsymbol{e}}_{x}, \overline{\boldsymbol{e}}_{y}, \overline{\boldsymbol{e}}_{z}\right]$ [43],

$$
\begin{aligned}
\varepsilon_{1} & =\bar{u}_{x}^{q}, \\
\varepsilon_{2} & =l_{0} \bar{\varphi}_{x}^{q}, \\
\varepsilon_{3} & =-l_{0} \bar{\varphi}_{y}^{p}, \\
\varepsilon_{4} & =l_{0} \bar{\varphi}_{y}^{q}, \\
\varepsilon_{5} & =-l_{0} \bar{\varphi}_{z}^{p}, \\
\varepsilon_{6} & =l_{0} \bar{\varphi}_{z}^{q} .
\end{aligned}
$$

From a comparison with Eq. (3.6c) it can be observed that,

$$
\overline{\boldsymbol{\mu}}_{\mathrm{e}}=\boldsymbol{A} \varepsilon
$$

where

$$
\boldsymbol{A}=\operatorname{diag}\left(\left[1, \frac{1}{l_{0}},-\frac{1}{l_{0}}, \frac{1}{l_{0}},-\frac{1}{l_{0}}, \frac{1}{l_{0}}\right]\right) .
$$

This can also be verified visually by comparing the deformation modes in Fig. 3.2 and Fig. 3.5.

\section{Equivalent stiffness matrix}

Substituting Eqs. (3.18a) and (3.8) in Eq. (3.7) and equating the result with Eq. (3.16) yields,

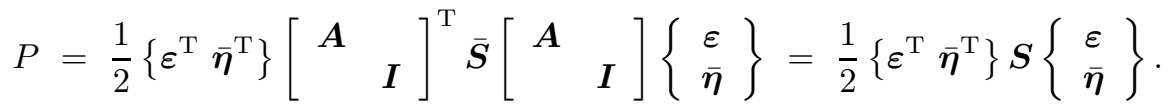

From Eq. (3.19) the equivalent stiffness matrix $\boldsymbol{S}$ can be identified as

$$
\boldsymbol{S}=\left[\begin{array}{ll}
\boldsymbol{A} & \\
& \boldsymbol{I}
\end{array}\right]^{\mathrm{T}} \overline{\boldsymbol{S}}\left[\begin{array}{ll}
\boldsymbol{A} & \\
& \boldsymbol{I}
\end{array}\right]
$$

The stiffness matrix $\boldsymbol{S}$ is symmetric and remains constant during a simulation. Note that the stiffness matrix $\boldsymbol{S}$ can directly be derived from a FE model created with a FE software package such as Ansys.

\subsubsection{Kinetic energy}

\section{Relating velocities}

With an expression for the kinetic energy of the non-linear superelement the inertial forces acting on the superelement can be determined. The kinetic energy is expressed 
in terms of the nodal coordinate time derivatives $\dot{\boldsymbol{x}}$ and the time derivatives of the set of generalized coordinates $\dot{\bar{\eta}}$,

$$
T=\frac{1}{2}\left\{\dot{\boldsymbol{x}}^{\mathrm{T}} \dot{\overline{\boldsymbol{\eta}}}^{\mathrm{T}}\right\} \boldsymbol{M}\left\{\begin{array}{c}
\dot{\boldsymbol{x}} \\
\dot{\overline{\boldsymbol{\eta}}}
\end{array}\right\} .
$$

where $M$ is the mass matrix of the non-linear superelement and

$$
\dot{\boldsymbol{x}}=\left\{\dot{\boldsymbol{r}}^{p \mathrm{~T}}, \quad \dot{\boldsymbol{\lambda}}^{p \mathrm{~T}}, \quad \dot{\boldsymbol{r}}^{q \mathrm{~T}}, \quad \dot{\boldsymbol{\lambda}}^{q \mathrm{~T}}\right\}^{\mathrm{T}} .
$$

Similar to the approach used in Section 3.3.2, an expression for the mass matrix can be obtained by relating the kinetic energy of Eq. (3.21) with the kinetic energy of the linear superelement given by Eq. (3.10). Here we observe in both kinetic energy expressions the same set of velocities $\dot{\bar{\eta}}$. Relating the kinetic energies then requires relating the time derivatives of the nodal coordinates $\dot{\boldsymbol{x}}$ with the boundary velocities $\overline{\boldsymbol{v}}$, whose components are expressed along the base vectors $\overline{\boldsymbol{e}}_{x}, \overline{\boldsymbol{e}}_{y}$ and $\overline{\boldsymbol{e}}_{z}$ (see Fig. 3.1).

Using Euler parameter identities and Eq. (3.22), the $14 \times 1$ vector $\dot{\boldsymbol{x}}$ can be expressed in terms of the $12 \times 1$ vector $\boldsymbol{v}$ of absolute nodal velocities expressed in the inertial reference frame, as

$$
\boldsymbol{v}=\left\{\begin{array}{c}
\boldsymbol{v}^{p} \\
\boldsymbol{\omega}^{p} \\
\boldsymbol{v}^{q} \\
\boldsymbol{\omega}^{q}
\end{array}\right\}=\left[\begin{array}{llll}
\boldsymbol{I} & & & \\
& 2 \boldsymbol{\Lambda}^{p} & & \\
& & \boldsymbol{I} & \\
& & & 2 \boldsymbol{\Lambda}^{q}
\end{array}\right]\left\{\begin{array}{c}
\dot{\boldsymbol{r}}^{p} \\
\dot{\boldsymbol{\lambda}}^{p} \\
\dot{\boldsymbol{r}}^{q} \\
\dot{\boldsymbol{\lambda}}^{q}
\end{array}\right\}
$$

where $\boldsymbol{v}^{p}$ and $\boldsymbol{v}^{q}$ are the absolute nodal translational velocities and $\boldsymbol{\omega}^{p}$ and $\boldsymbol{\omega}^{q}$ are the absolute nodal angular velocities respectively defined by

$$
\boldsymbol{\omega}^{p}=2 \boldsymbol{\Lambda}^{p} \dot{\boldsymbol{\lambda}}^{p} \quad \text { and } \quad \boldsymbol{\omega}^{q}=2 \boldsymbol{\Lambda}^{q} \dot{\boldsymbol{\lambda}}^{q} .
$$

Here the matrices $\boldsymbol{\Lambda}^{p}$ and $\boldsymbol{\Lambda}^{q}$ are defined by the Euler parameters $\boldsymbol{\lambda}^{p}$ and $\boldsymbol{\lambda}^{q}$ and are of the following form:

$$
\boldsymbol{\Lambda}=\left[\begin{array}{rrrr}
-\lambda_{1} & \lambda_{0} & -\lambda_{3} & \lambda_{2} \\
-\lambda_{2} & \lambda_{3} & \lambda_{0} & -\lambda_{1} \\
-\lambda_{3} & -\lambda_{2} & \lambda_{1} & \lambda_{0}
\end{array}\right]
$$

with $\lambda_{0}, \lambda_{1}, \lambda_{2}$ and $\lambda_{3}$ being the four Euler parameters of a vector $\boldsymbol{\lambda}$.

In [12] (Chapter 2) it is shown by a comparison with the floating frame of reference approach [62] that the velocity vector $\overline{\boldsymbol{v}}$, when assuming infinitesimal elastic deformations, can be obtained by expressing the components of the absolute nodal velocities $\boldsymbol{v}$ along the axes of the co-rotational frame $\left[\overline{\boldsymbol{e}}_{x}, \overline{\boldsymbol{e}}_{y}, \overline{\boldsymbol{e}}_{z}\right]$. This transformations requires an expression for the co-rotational frame in the inertial reference frame. For infinitesimal elastic deformations, either one of the orthonormal triads $\left[\boldsymbol{e}_{x}^{p}, \boldsymbol{e}_{y}^{p}, \boldsymbol{e}_{z}^{p}\right]$ and $\left[\boldsymbol{e}_{x}^{q}, \boldsymbol{e}_{y}^{q}, \boldsymbol{e}_{z}^{q}\right]$ of Eq. (3.12) can define the co-rotational frame. However, in [12] we proposed an approach inspired by Cardona [21] that produces more accurate and robust results for small elastic deformations while 
being exact when elastic deformations are absent. Here, the components of the angular velocities are expressed along the axes of their respective orthonormal triads $\left[\boldsymbol{e}_{x}^{p}, \boldsymbol{e}_{y}^{p}, \boldsymbol{e}_{z}^{p}\right]$ and $\left[\boldsymbol{e}_{x}^{q}, \boldsymbol{e}_{y}^{q}, \boldsymbol{e}_{z}^{q}\right]$, while the translational velocities are expressed in an average orientation reference frame. The average orientation reference frame can be computed from the average set of Euler parameters $\boldsymbol{\lambda}^{r}$ defined by

$$
\boldsymbol{\lambda}^{r}=\frac{\boldsymbol{\lambda}^{p}+\boldsymbol{\lambda}^{q}}{\left\|\boldsymbol{\lambda}^{p}+\boldsymbol{\lambda}^{q}\right\|}
$$

where the differences between $\boldsymbol{\lambda}^{p}$ and $\boldsymbol{\lambda}^{q}$ are assumed to be small. The Euler parameters $\lambda^{r}$ represents the rotation matrix $\boldsymbol{R}^{r}$ which describes the average orientation with respect to the initial orientation $\boldsymbol{R}_{0}$ of Eq. (3.12). The relation between the velocity vectors $\boldsymbol{v}$ and $\overline{\boldsymbol{v}}$ is then given by

$$
\overline{\boldsymbol{v}}=\left[\begin{array}{cccc}
\boldsymbol{R}_{0}^{\mathrm{T}} \boldsymbol{R}^{r \mathrm{~T}} & & & \\
& \boldsymbol{R}_{0}^{\mathrm{T}} \boldsymbol{R}^{p \mathrm{~T}} & & \\
& & \boldsymbol{R}_{0}^{\mathrm{T}} \boldsymbol{R}^{r \mathrm{~T}} & \\
& & & \boldsymbol{R}_{0}^{\mathrm{T}} \boldsymbol{R}^{q \mathrm{~T}}
\end{array}\right] \boldsymbol{v} .
$$

Substituting Eq. (3.23) in (3.27) then gives for the relation between the nodal coordinate time derivatives $\dot{\boldsymbol{x}}$ and the velocity vector $\overline{\boldsymbol{v}}$,

$$
\overline{\boldsymbol{v}}=\boldsymbol{B} \dot{\boldsymbol{x}}
$$

where

$$
\boldsymbol{B}=\left[\begin{array}{cccc}
\boldsymbol{R}_{0}^{\mathrm{T}} \boldsymbol{R}^{r \mathrm{~T}} & & & \\
& 2 \boldsymbol{R}_{0}^{\mathrm{T}} \boldsymbol{R}^{p \mathrm{~T}} \boldsymbol{\Lambda}^{p} & & \\
& & \boldsymbol{R}_{0}^{\mathrm{T}} \boldsymbol{R}^{r \mathrm{~T}} & \\
& & & 2 \boldsymbol{R}_{0}^{\mathrm{T}} \boldsymbol{R}^{q \mathrm{~T}} \boldsymbol{\Lambda}^{q}
\end{array}\right]
$$

\section{Equivalent mass matrix}

Substituting Eqs. (3.28) and (3.11) in (3.10) and equating the result with Eq. (3.21) yields

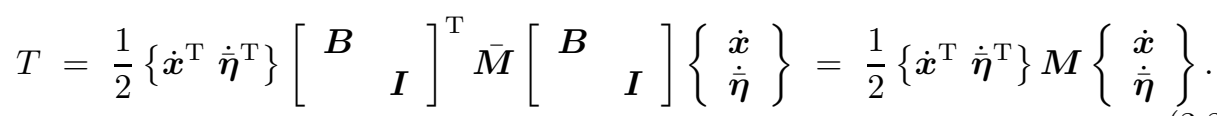

from which it can be observed that the equivalent mass matrix $M$ is given by

$$
\boldsymbol{M}=\left[\begin{array}{ll}
\boldsymbol{B} & \\
& \boldsymbol{I}
\end{array}\right]^{\mathrm{T}} \overline{\boldsymbol{M}}\left[\begin{array}{cc}
\boldsymbol{B} & \\
& \boldsymbol{I}
\end{array}\right]=\left[\begin{array}{cc}
\boldsymbol{B}^{\mathrm{T}} \overline{\boldsymbol{M}}_{\mu \mu} \boldsymbol{B} & \boldsymbol{B}^{\mathrm{T}} \overline{\boldsymbol{M}}_{\mu \eta} \\
\overline{\boldsymbol{M}}_{\eta \mu} \boldsymbol{B} & \overline{\boldsymbol{M}}_{\eta \eta}
\end{array}\right],
$$

where the subscripts $\mu$ and $\eta$ indicate the parts of the mass matrix $\overline{\boldsymbol{M}}$ associated with the boundary coordinates $\overline{\boldsymbol{\mu}}$ and the generalized coordinates $\overline{\boldsymbol{\eta}}$. The velocity transformation matrix $\boldsymbol{B}$ is dependent on the superelement configuration described by the nodal coordinates $\boldsymbol{x}$ and as a consequence, the mass matrix $\boldsymbol{M}$ is also configuration dependent. The matrix $\overline{\boldsymbol{M}}$ remains constant during simulation and can directly be determined from a linear FE model, see Eq. (3.11). 


\subsubsection{Dynamics}

We can determine the forces acting on the superelement by considering the principle of virtual work. The virtual work done by external forces $f_{\text {ext }}$ should be equal to the virtual work of the inertial forces $\boldsymbol{f}_{\text {in }}$ and the stress resultants $\boldsymbol{\sigma}$,

$$
\left\{\begin{array}{l}
\delta \boldsymbol{x} \\
\delta \overline{\boldsymbol{\eta}}
\end{array}\right\}^{\mathrm{T}} \boldsymbol{f}_{\mathrm{ext}}=-\left\{\begin{array}{l}
\delta \boldsymbol{x} \\
\delta \overline{\boldsymbol{\eta}}
\end{array}\right\}^{\mathrm{T}} \boldsymbol{f}_{\mathrm{in}}+\left\{\begin{array}{l}
\delta \varepsilon \\
\delta \overline{\boldsymbol{\eta}}
\end{array}\right\}^{\mathrm{T}} \boldsymbol{\sigma}
$$

With the aid of Lagrange's equations, the virtual work of the inertial forces and stress resultants can also be expressed in terms of potential and kinetic energy,

$$
\left\{\begin{array}{l}
\delta \boldsymbol{x} \\
\delta \overline{\boldsymbol{\eta}}
\end{array}\right\}^{\mathrm{T}} \boldsymbol{f}_{\mathrm{ext}}=\left\{\begin{array}{l}
\delta \boldsymbol{x} \\
\delta \overline{\boldsymbol{\eta}}
\end{array}\right\}^{\mathrm{T}}\left(\frac{d}{d t}\left(\frac{\partial T}{\partial(\dot{\boldsymbol{x}}, \dot{\bar{\eta}})}\right)-\frac{\partial T}{\partial(\boldsymbol{x}, \overline{\boldsymbol{\eta}})}+\frac{\partial P}{\partial(\boldsymbol{x}, \overline{\boldsymbol{\eta}})}\right)^{\mathrm{T}} .
$$

Substituting the potential and kinetic energy expressions of Eqs. (3.19) and (3.30) in Eq. (3.33) and evaluating the derivatives gives

$$
\left\{\begin{array}{l}
\delta \boldsymbol{x} \\
\delta \overline{\boldsymbol{\eta}}
\end{array}\right\}^{\mathrm{T}} \boldsymbol{f}_{\mathrm{ext}}=\left\{\begin{array}{l}
\delta \boldsymbol{x} \\
\delta \overline{\boldsymbol{\eta}}
\end{array}\right\}^{\mathrm{T}}\left(\boldsymbol{M}\left\{\begin{array}{l}
\ddot{\boldsymbol{x}} \\
\ddot{\boldsymbol{\eta}}
\end{array}\right\}+\boldsymbol{h}\right)+\left\{\begin{array}{l}
\delta \varepsilon \\
\delta \overline{\boldsymbol{\eta}}
\end{array}\right\}^{\mathrm{T}} \boldsymbol{S}\left\{\begin{array}{l}
\boldsymbol{\varepsilon} \\
\overline{\boldsymbol{\eta}}
\end{array}\right\}
$$

where the vector $\boldsymbol{h}$ contains the inertial forces that are quadratic with velocity,

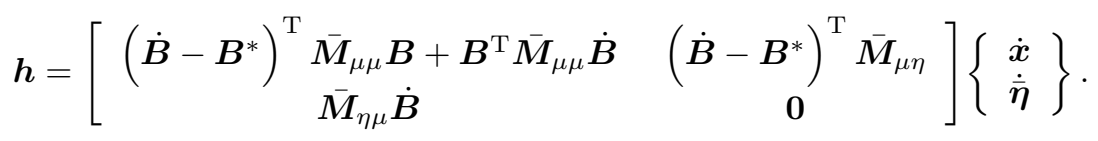

Here the matrix $\boldsymbol{B}^{*}$ is defined as

$$
\boldsymbol{B}^{*}=\frac{\partial \overline{\boldsymbol{v}}}{\partial \boldsymbol{x}}
$$

For expressions for $\dot{\boldsymbol{B}}$ and $\boldsymbol{B}^{*}$ see Appendices B and C. By comparing Eq. (3.32) with Eq. (3.34a), the inertial forces and generalized stress resultants are expressed as

$$
f_{\text {in }}=-\left(M\left\{\begin{array}{c}
\ddot{x} \\
\ddot{\eta}
\end{array}\right\}+h\right) \quad \text { and } \quad \boldsymbol{\sigma}=\boldsymbol{S}\left\{\begin{array}{c}
\varepsilon \\
\bar{\eta}
\end{array}\right\} .
$$

In order to use the non-linear superelement in flexible multibody models, the expressions in Eq. (3.36) can be included in the equations of motion of the entire multibody system ${ }^{1}$. An implementation of these expressions is realized in a $\mathrm{C}++$ version of the SPACAR flexible multibody software [39]. This program can make computations for mechanical systems with interconnected rigid and flexible elements. Specifically, the motion can be simulated for given initial conditions, the equations of motion can be linearized about an arbitrary state of motion, stationary solutions can be determined, and with the linearized equations, eigenfrequencies and corresponding vibrational modes can be determined.

\footnotetext{
${ }^{1}$ See Appendix D for the equations of motion of a multibody system with superelements.
} 


\subsection{Superelements with deformable-interface modes}

In this section we will address the connection between superelements with deformableinterface modes.

\subsubsection{Assembly of superelements}

Element connectivity can be realized by letting elements share their nodal coordinates. In a similar manner, the coordinates $\overline{\boldsymbol{\eta}}_{d}^{p}$ and $\overline{\boldsymbol{\eta}}_{d}^{q}$ of Eq. (3.15) can be shared as well. Though, care has to be taken when connecting superelements with deformable-interface modes. For a proper connection, it should be verified that the interface deformations of a deformable-interface mode are exactly the same for the connecting superelements. In other words, the deformable-interface modes should be compatible. In Fig. 3.6(a) this is exemplified for two interconnected superelements representing a tube with an open cross-section clamped at one end and subjected to a torsional rotation at the other end. Here, the warping modes of the superelements are compatible resulting in a correct connection. This is however not always the case for warping modes computed with the approach described in Section 3.2.1, which can be observed in Fig. 3.6(b) where two interconnected superelements with different shear moduli yield a separation of the interface surfaces when subjected to a torsional rotation. Because the warping modes are computed with FE analyses, the geometry and material properties will play a role in determining the warping deformations of the interface surfaces. Compatibility of the warping modes can therefore only be guaranteed for superelements that have identical material properties and whose geometries are mirror symmetric with respect to their shared interface surface.

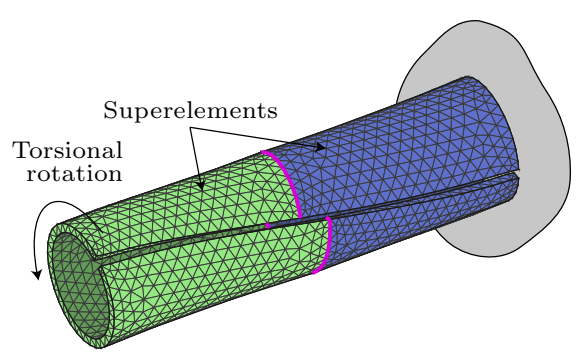

(a) Compatible warping modes

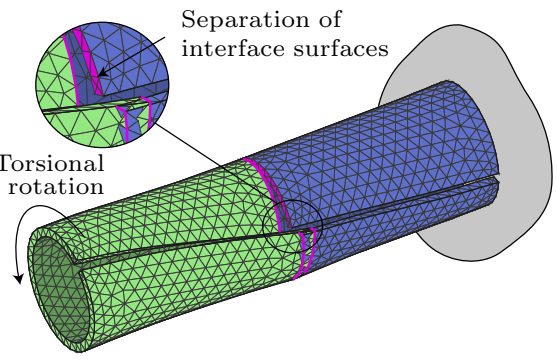

(b) Incompatible warping modes due to a different shearing modulus for each superelement

Figure 3.6: Torsion of a tube with open cross-section modelled by two superelements with compatible (a) and incompatible warping modes (b). 


\subsubsection{Determining compatible warping modes}

When the geometries are not mirror symmetric or if the material properties are different, an alternative strategy has to be followed for obtaining compatible warping modes.

Consider the tube shown in Fig. 3.7(a) which consists of two sections, $A$ and $B$. Each section should be modelled by a superelement and their shared interface should be able to deform for a torsional twist of the tube. The steps that may be followed to obtain the compatible warping modes are presented below:

1. Generate a FE model that consists of sections $A$ and $B$ and rigidly prescribe a torsional rotation to one end of the tube while clamping the other end and compute the resulting deformations, see Fig. 3.7(a).

2. Determine from this FE analysis the nodal displacements on the interface between sections $\mathrm{A}$ and $\mathrm{B}$ and collect them in the $3 n \times 1$ vector $\overline{\boldsymbol{u}}_{\text {interface }}$, where $n$ corresponds to the number of nodes on the interface.

3. Remove the interface surface rigid body motion from the vector $\overline{\boldsymbol{u}}_{\text {interface }}$. Let the rigid body motion of the interface surface be represented by the $3 n \times 6$ matrix $\boldsymbol{U}_{r}$ whose columns are normalized and correspond to the $x, y$ and $z$ translations and rotations of the interface surface, then

$$
\overline{\boldsymbol{u}}_{\text {interface }}^{d}=\overline{\boldsymbol{u}}_{\text {interface }}-\boldsymbol{U}_{r} \boldsymbol{U}_{r}^{\mathrm{T}} \overline{\boldsymbol{u}}_{\text {interface }},
$$

where $\overline{\boldsymbol{u}}_{\text {interface }}^{d}$ are the interface deformations absent rigid body motion of the interface surface.

4. Obtain from a FE analysis of section $A$ the warping mode $\boldsymbol{W}_{d}^{q(A)}$ by clamping the nodes at the $p$-side of section $A$ and applying the interface deformations $\overline{\boldsymbol{u}}_{\text {interface }}^{d}$ to the nodes at the $q$-side, see Fig. 3.7(b).

5. Obtain in a similar manner the warping mode $\boldsymbol{W}_{d}^{p(B)}$ from a FE model of section $B$, by applying the interface deformations $\overline{\boldsymbol{u}}_{\text {interface }}^{d}$ to the nodes at the $p$-side while clamping the $q$-side, see Fig. 3.7(b).

The removal of the rigid body motion from the vector $\overline{\boldsymbol{u}}_{\text {interface }}$ in step 3 is necessary to ensure that the warping modes computed in steps 4 and 5 are independent of the constraint modes. The computed warping modes in steps 4 and 5 are compatible as they describe the same deformations $\overline{\boldsymbol{u}}_{\text {interface }}^{d}$ at the shared interface surface. Furthermore, with these warping modes it is ensured that a correct static response of the interface surface is obtained for torsional rotations of the tube.

Note that this method can also be used to obtain other types of compatible deformable-interface modes attuned to a different displacement pattern prescribed in step 1. For example, by prescribing, instead of the torsional rotation, a rigid translation of the interface surface along the element axis in step 1, a deformableinterface mode is obtained that models the transverse contraction at the interface due to elongation. Though, for additional deformable-interface modes, it should be 


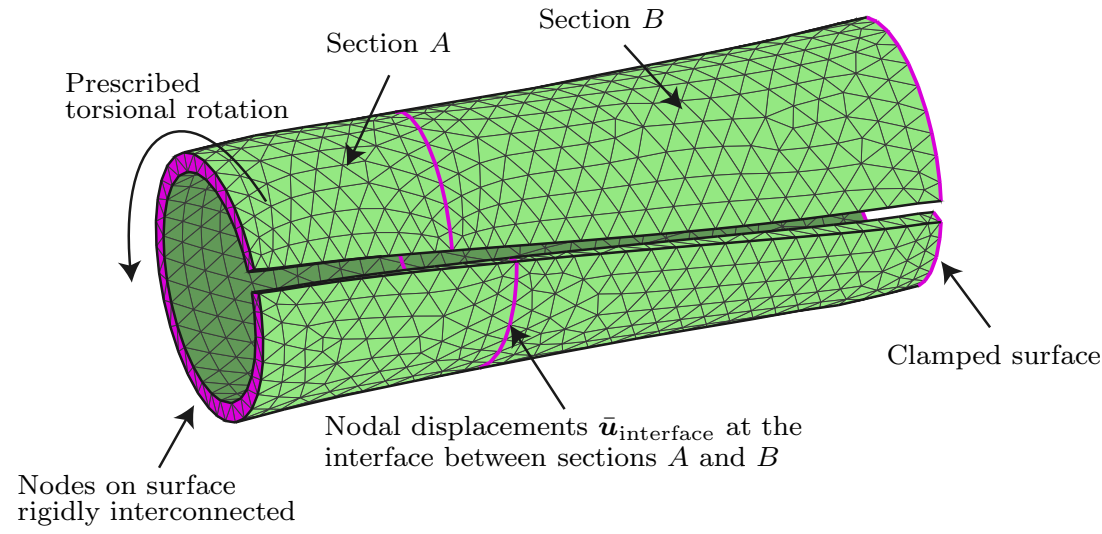

(a) FE model of a tube with open-cross-section consisting of two sections, $A$ and $B$ Compatible warping mode $\boldsymbol{W}_{d}^{p(B)}$

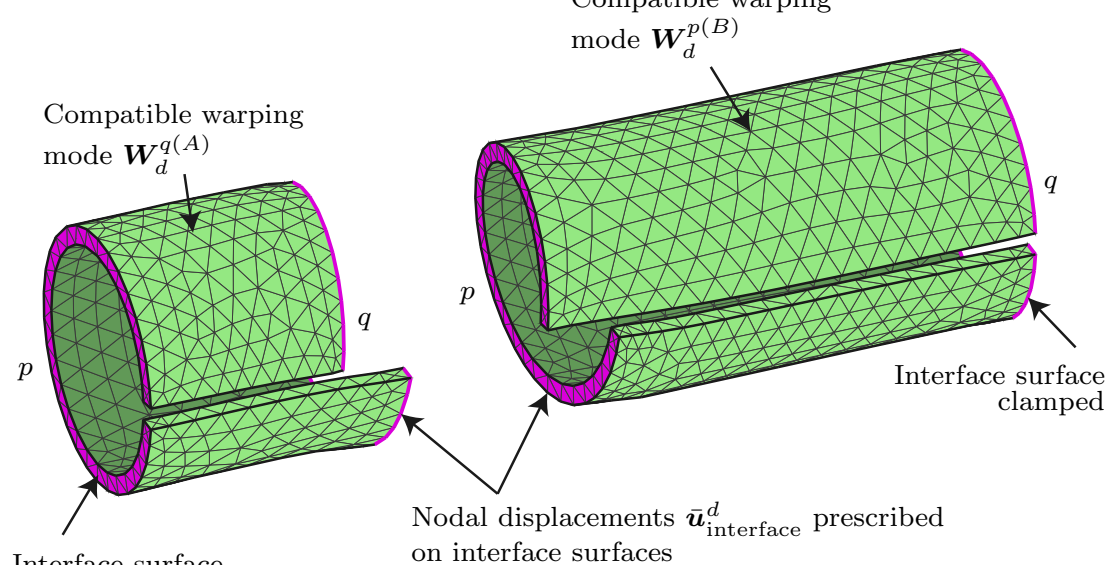

Interface surface on interface surfaces

(b) Computation of compatible warping modes for sections $A$ and $B$

Figure 3.7: Computation of compatible warping modes by obtaining interface nodal displacements from a FE model (a) and prescribing these to the interface surfaces of the separate sections (b).

verified that they are linearly independent to obtain full rank mass and stiffness matrices for the superelement (see Sections 3.2.2 and 3.2.3).

\subsection{Numerical examples}

Figure 3.8 shows a complex-shaped flexible component attached to a compliant cross-hinge consisting of sheet flexures. This assembly is part of a large-stroke compliant mechanism [27]. We will use a simplified model of this assembly to 


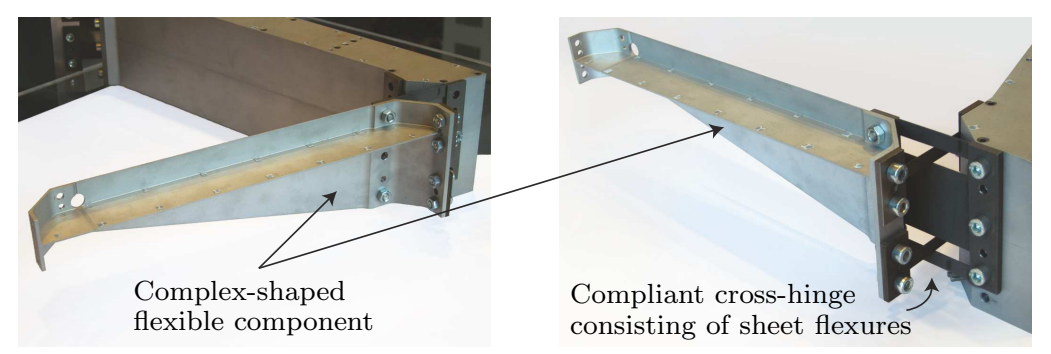

Figure 3.8: Images of a complex-shaped flexible component attached to a compliant cross-hinge consisting of sheet flexures. The component and flexures are part of a large-stroke compliant mechanism [27].

demonstrate the performance of the non-linear two-node superelement. Three separate examples are considered to test different aspects of the superelement. In the first example, constraint and warping modes are combined for the modelling of large deflections of a short sheet flexure. Next, a complex-shaped flexible component which only exhibits small deflections is modelled by a single superelement with additional fixed-interface normal modes. For the third example, a time-response simulation is performed of the motion of the complex-shaped component of the second example connected to the fixed world by a compliant cross-hinge consisting of short sheet flexures. For all examples, we compute eigenfrequencies in different equilibrium configurations and compare the results with those obtained using the FE software ANsys. Stress stiffening effects are included for the computation of the eigenfrequencies $[40]^{2}$.

\subsubsection{Large deflections of a compliant flexure}

Consider the flexure shown in Fig. 3.9. On one side it is clamped to the fixed world, whereas on the other side a nodal mass $m$ is attached to the flexure using an L-shaped rigid link with dimensions in the $x$ and $y$-direction of respectively $0.25 \mathrm{~m}$ and $0.1 \mathrm{~m}$. Both ends of the flexure are considered to be rigid over the full height of the flexure, i.e. warping is constrained. The height $h$, length $l$, and thickness $t$ are denoted in the figure as are its physical properties corresponding to steel with density $\rho$, Young's modulus $E$ and shear modulus $G$. At the connection of the flexure with the rigid link, a moment $M$ is applied such that the flexure is deflected over an angle $\varphi$.

In this example we consider four models for computing the first four eigenfrequencies and vibrational modes as functions of the deflection angle $\varphi$. The first two models are superelement models. The first superelement model only includes constraint modes whereas for the second superelement model also warping modes are considered. These models will respectively be referred to by the labels SE-C and

\footnotetext{
${ }^{2}$ See Appendix E for the determination of the mass and stiffness matrices that are used to compute the eigenfrequencies.
} 

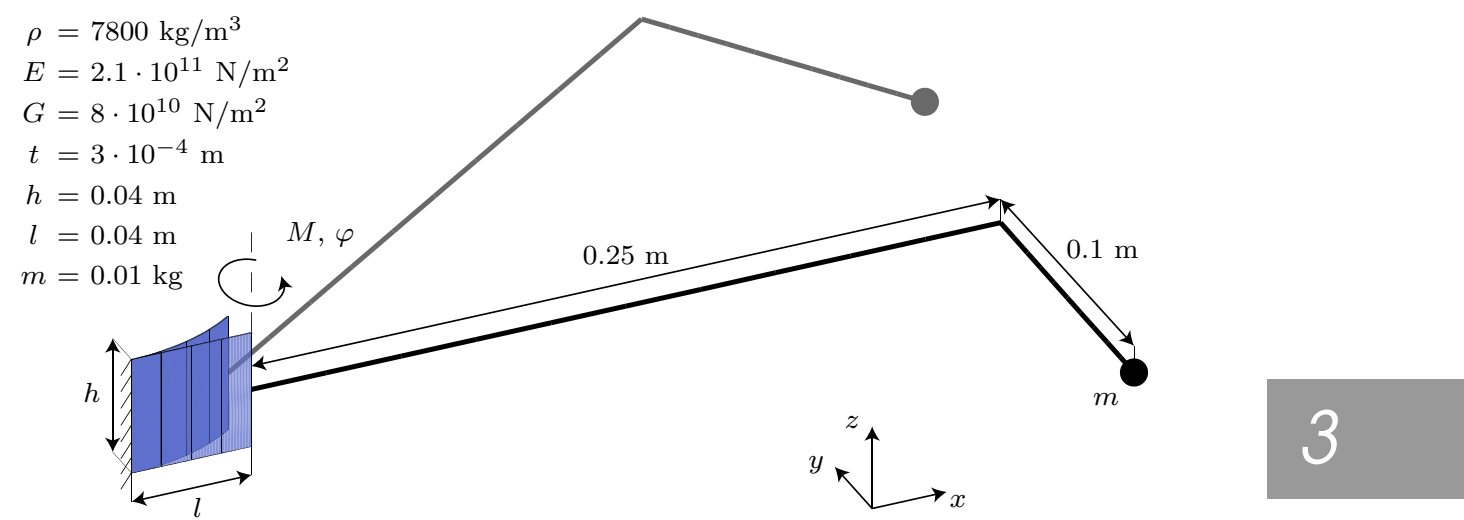

Figure 3.9: Parameters of the flexure model with a nodal mass attached to it.

SE-CW. The third model consists of SPACAR non-linear beam elements [43] and will be abbreviated as NLB-SPACAR. Lastly, as reference, we performed a non-linear large deflection analysis using the FE software ANSYs and we will refer to the results of this model as FE-ANSYs. Each of the models is investigated in 40 equilibrium configurations obtained by applying a moment of $\pm 0.4 \mathrm{Nm}$ to the flexure with increments of $0.02 \mathrm{Nm}$ yielding a total deflection of $\varphi= \pm 40$ degrees.

For the modelling of large deflections using beams or superelements, it is required that the element deformations remain relatively small such that linear stiffness models are valid. Therefore, the flexure needs to be discretized with a sufficient number of elements along its length to produce correct large deflection results. We used a total of four beam elements and four superelements for the non-linear beam and superelement models respectively. For the NLB-SPACAR and SE-C model this leads to models with 24 degrees of freedom. The SE-CW model has three additional degrees of freedom to model the warping of the flexure. The warping at the ends of the flexure is suppressed to model the clamping and the rigid connection to the rigid link. The superelements are obtained from a FE model of a quarter of the flexure consisting of 1600, 20-node solid elements (ANSYs Solid 186 elements) in cubic configuration with four elements across the thickness of the flexure. For the FE Ansys model, 6400, 20-node solid elements are used with four elements across the thickness of the flexure. Furthermore, to realize a rigid connection of the rigid link to the flexure, the nodes over the full height at the end of the flexure are rigidly interconnected using Ansys multipoint constraint elements.

The first four eigenfrequencies of the four models are shown in Fig. 3.10 as functions of the deflection angle $\varphi$. All four models are in good agreement for the first and third eigenfrequencies. Though, large differences for the second and fourth eigenfrequencies are observed for the NLB-SPACAR and the SE-C model. The second vibrational mode is a torsion mode and the fourth vibrational mode can also be 


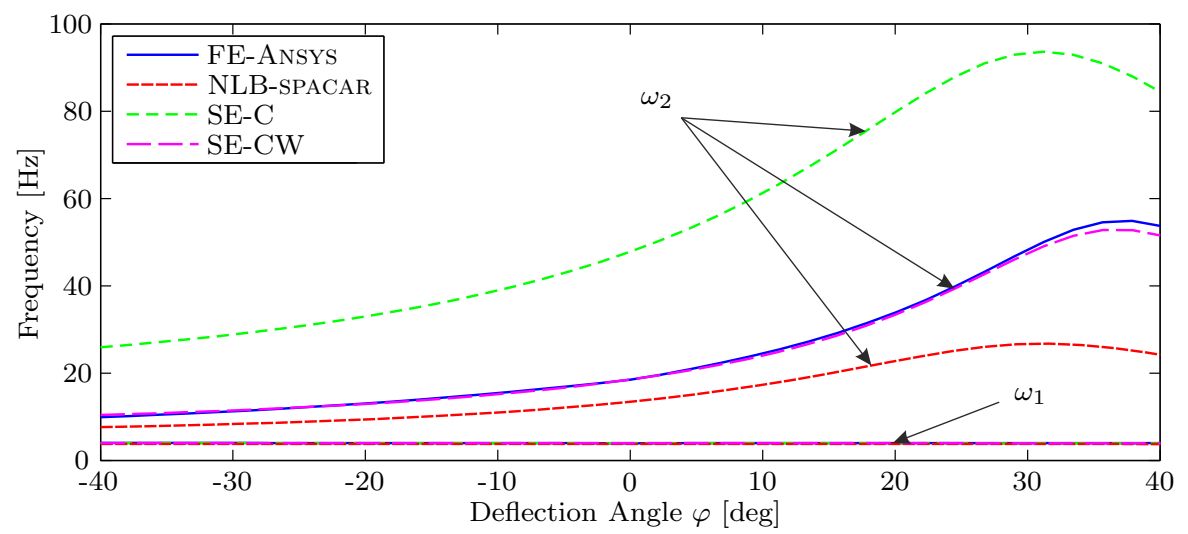

(a) The first and second eigenfrequencies, $\omega_{1}$ and $\omega_{2}$, as a function of the deflection angle

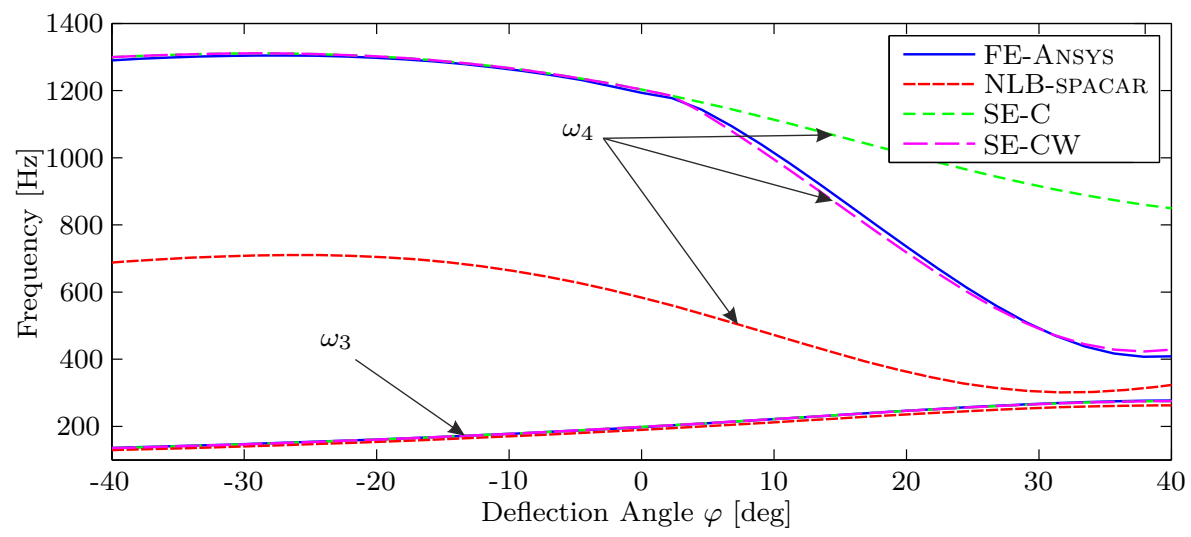

(b) The third and fourth eigenfrequencies, $\omega_{3}$ and $\omega_{4}$, as a function of the deflection angle

Figure 3.10: The first two eigenfrequencies (a) and the third and fourth eigenfrequencies (b) as a function of the deflection angle for the four considered models.

considered a torsion mode for positive deflections, see Fig. 3.11(a). The ends of the flexure would like to warp under a torsional rotation as is discussed in Section 3.2.1. This is especially the case for short flexures such as the one considered in this example. By clamping the flexure at one end and rigidly attaching it to the rigid link on the other end, the warping is suppressed on both sides of the flexure yielding a significant increase of the torsional stiffness. This effect is not modelled by the NLB-SPACAR model resulting in the much lower second and fourth eigenfrequency. For the SE-C model, each superelement is generated using rigid interfaces and therefore automatically suppresses the warping at both ends of each superelement. As we have used four superelements in the SE-C model, this means that the warping 


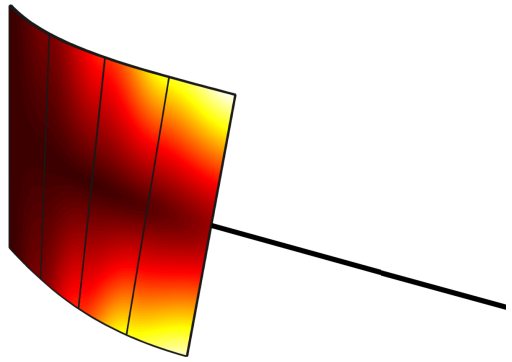

(a) Fourth vibrational mode for positive deflections

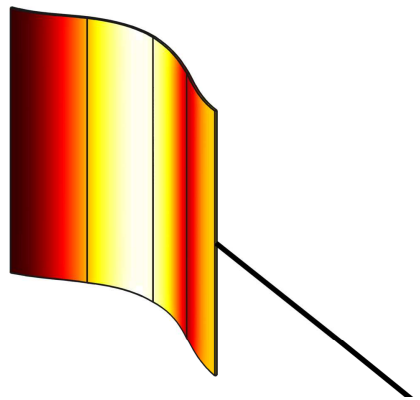

(b) Fourth vibrational mode for negative deflections

Figure 3.11: The fourth vibrational mode of the flexure for positive deflections (a) and for negative deflections (b).

is suppressed correctly at both sides of the flexure but also at a quarter, a half and three quarter lengths of the flexure, explaining the much higher second and fourth eigenfrequencies. From Fig. 3.11(b), we observe that for negative deflections the fourth vibrational mode does not correspond to a torsional mode but is instead a bending mode. For the negative deflections, the torsional mode has a higher frequency than the bending mode and they start to veer with each other around 2 degrees deflection. The bending mode is predicted well by the SE-C model yielding the correct results of the fourth eigenfrequency for negative deflections. The NLB-SPACAR model also predicts this mode, but as its sixth vibrational mode (not shown in the figure).

Correct results are obtained for the SE-CW model over the full range of motion. From this we can conclude that the warping effect is correctly modelled within the flexure, while constrained at the ends of the flexure. It is clear that the inclusion of warping modes is of importance for the correct modelling of short flexures using superelements.

\subsubsection{Modelling of a complex-shaped flexible component exhibiting small deflections}

For the modelling of a complex-shaped flexible component exhibiting only small deflections, a single superelement with additional fixed-interface normal modes can suffice. In this example we demonstrate this for the flexible component shown in Fig. 3.12 .

The flexible component is attached to a nodal mass $m$ by a rigid link and it is clamped on its other side. All the dimensions of the component and its physical properties are shown in the figure. We will model this component using a two-node superelement and rigidly attach it to the nodal mass. The first six eigenfrequencies 


$$
\begin{aligned}
t & =0.0015 \mathrm{~m} \\
h^{p} & =0.08 \mathrm{~m} \\
w^{p} & =0.03 \mathrm{~m} \\
h^{q} & =0.016 \mathrm{~m} \\
w^{q} & =0.006 \mathrm{~m}
\end{aligned}
$$

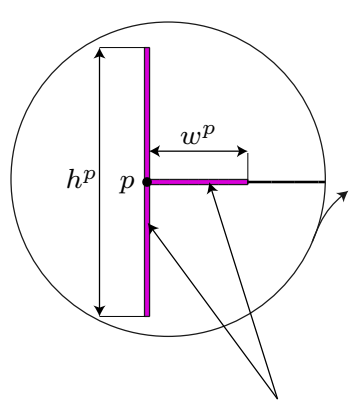

Rigid $p$-side interface surface

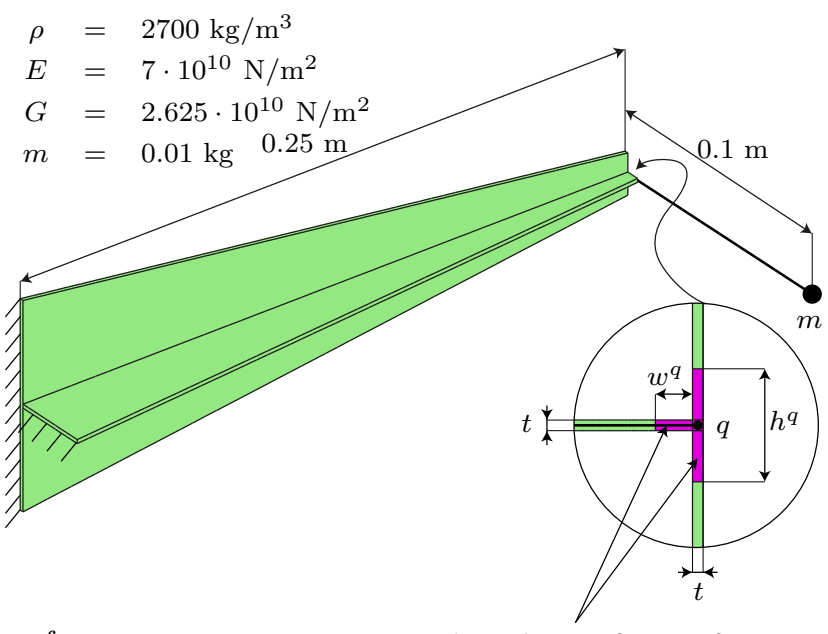

Rigid $q$-side interface surface

Figure 3.12: Parameters of a flexible component attached to a nodal mass.

Table 3.1: The first six eigenfrequencies for the FE Ansys model and the superelement models with constraint (SE-C) and additionally one, two and six fixed-interface normal modes (SE-CF1, SE-CF2 and SE-CF6).

\begin{tabular}{llllll}
\hline & FE ANSYS $[\mathrm{Hz}]$ & SE-C $[\mathrm{Hz}]$ & SE-CF1 $[\mathrm{Hz}]$ & SE-CF2 $[\mathrm{Hz}]$ & SE-CF6 $[\mathrm{Hz}]$ \\
\hline & & 41.4 & 41.4 & 41.4 & 41.4 \\
Mode 1 & 41.4 & 199.5 & 199.5 & 199.5 & 199.4 \\
Mode 2 & 199.4 & 395.3 & 395.3 & 395.3 & 389.5 \\
Mode 3 & 389.2 & - & 438.6 & 438.6 & 438.6 \\
Mode 4 & 438.6 & - & - & 949.7 & 949.6 \\
Mode 5 & 948.8 & 1132.5 & 1132.6 & 1139.0 & 1128.5 \\
Mode 6 & 1080.4 & \multicolumn{5}{c}{} \\
\hline
\end{tabular}

will be compared with those obtained from an ANSYS FE modal analysis. For this analysis we modelled the interface surfaces rigidly as is shown in the figure.

It is investigated how the eigenfrequencies are influenced by including fixedinterface normal modes to the superelement model. In Tab. 3.1 the eigenfrequency results are shown for the $\mathrm{FE}$ analysis and superelement models with varying number of fixed-interface normal modes. Here the labels SE-C, SE-CF1, SE-CF2 and SECF6 respectively refer to superelement models with only constraint and additionally one, two, or six fixed-interface normal modes. It can be observed that for a superelement with just constraint modes (SE-C), the first three eigenfrequencies are already predicted accurately. Though for predicting the fourth and fifth mode, two additional fixed-interface normal modes are necessary as is observed for the SE-CF1 and SE-CF2 models. Increasing the number of fixed-interface normal modes to six yields only a marginal increase in accuracy compared with the $\mathrm{FE}$ analysis. 


\subsubsection{Time-response of a complex-shaped flexible component attached to a compliant cross-hinge}

In Fig. 3.13, an illustration is presented of a simplified model of the compliant mechanism shown in Fig. 3.8. The numbers in the figure correspond to node numbers whose coordinates are given in Tab. 3.2. The short sheet flexure considered in the first example is placed between nodes 1 and 2 and between nodes 3 and 4. Each flexure is modelled by four superelements that include warping modes corresponding to the SE-CW model of the first example. Nodes 1 and 3 are clamped to the fixed world and the warping modes of the superelements are suppressed at nodes 1, 2, 3 and 4. The complex-shaped flexible component of the second example is placed between nodes 5 and 6 . This component is modelled by a single superelement with six fixed-interface normal modes corresponding to the SE-CF6 model of the second example. Similar to the previous examples, a nodal mass $m$ of $0.01 \mathrm{~kg}$ is attached to the mechanism by a massless rigid beam between nodes 6 and 7 . Furthermore, rigid beams are used to connect nodes 2 and 5, as well as nodes 4 and 5. The complete model has 60 degrees of freedom.

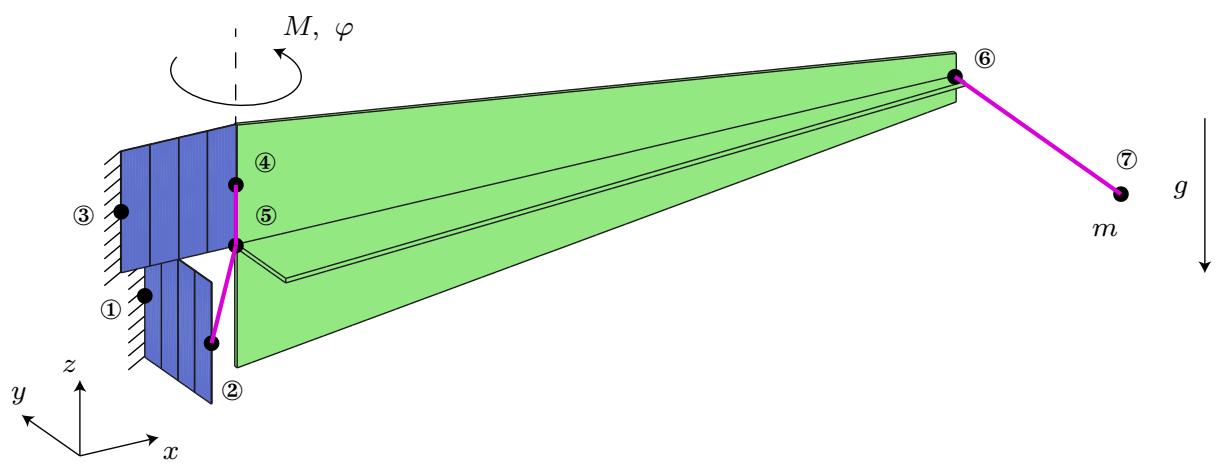

Figure 3.13: Compliant mechanism model consisting of two short sheet flexures and a complex-shaped flexible component. The circled numbers are node numbers of the model.

Table 3.2: The $x, y$ and $z$ coordinates of nodes 1 through 7 .

\begin{tabular}{llll}
\hline & $x$-coordinate $[\mathrm{m}]$ & $y$-coordinate $[\mathrm{m}]$ & $z$-coordinate $[\mathrm{m}]$ \\
\hline & & 0.02 & -0.02 \\
Node (1) & 0.02 & -0.02 & -0.02 \\
Node (2) & 0.02 & 0 & 0.02 \\
Node (3) & 0 & 0 & 0.02 \\
Node (4) & 0.04 & 0 & 0 \\
Node (5) & 0.04 & 0 & 0 \\
Node (6) & 0.29 & -0.1 & 0 \\
Node (7) & 0.29 & & \\
\hline
\end{tabular}




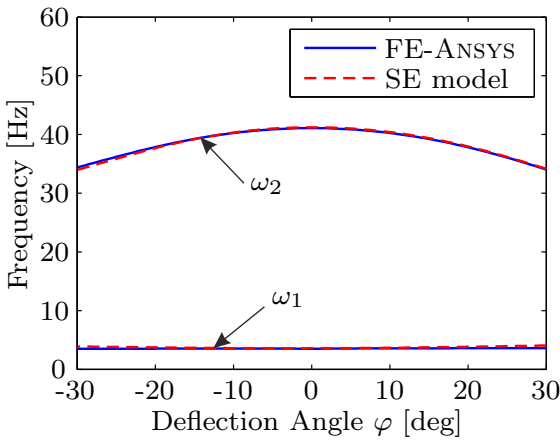

(a) Eigenfrequencies one and two

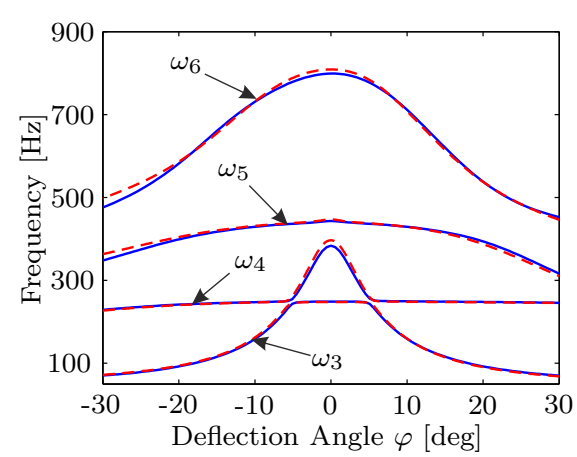

(b) Eigenfrequencies three through six

Figure 3.14: The first two eigenfrequencies $\omega_{1}$ and $\omega_{2}$ (a) and eigenfrequencies $\omega_{3}$ through $\omega_{6}$ (b) as functions of the deflection angle $\varphi$ of the superelement model compared with results obtained using ANSYs.

For the material properties of the flexures and the complex-shaped component see Fig. 3.9 and Fig. 3.12 respectively. A gravity field $g$ of $9.81 \mathrm{~m} / \mathrm{s}^{2}$ is acting in the negative $z$-direction for this example and a moment $M$ is applied to actuate the mechanism over a deflection angle $\varphi$.

Before performing the time-response simulation, we will validate the model by comparing the eigenfrequencies in 61 equilibrium positions with results obtained from a non-linear large deflection analysis using the FE software Ansys. We will omit the effects of gravity for this analysis. The equilibrium positions are determined by incrementing the moment $M$ by $0.02 \mathrm{Nm}$ up to $\pm 0.6 \mathrm{Nm}$. In Fig. 3.14 the first six eigenfrequencies are shown as functions of the deflection angle $\varphi$, up to $\varphi= \pm 30$ degrees. It can be concluded that the superelement model corresponds very well with the Ansys results.

To actuate the model for the time-response simulation, the moment $M$ is prescribed as a function of time

$$
M(t)= \begin{cases}\frac{M_{\max }}{2}\left(1-\cos \left(\frac{2 \pi t}{T}\right)\right), & 0 \leq t \leq T, \\ 0, & t>T,\end{cases}
$$

where the setup-time $T$ is $0.1 \mathrm{~s}$ and $M_{\max }$ is $0.5 \mathrm{Nm}$, see Fig. 3.15(a). Two timeresponse simulations are performed where the effects of gravity are included. The time-response simulations differ with respect to their initial conditions. The first time-response simulation starts from the original undeflected configuration, whereas the second time-response simulation starts in an adjusted equilibrium configuration that accounts for gravity. For the time-integration of the equations of motion we use the Shampine-Gordon integrator [63], which is an explicit variable step-size integrator. 


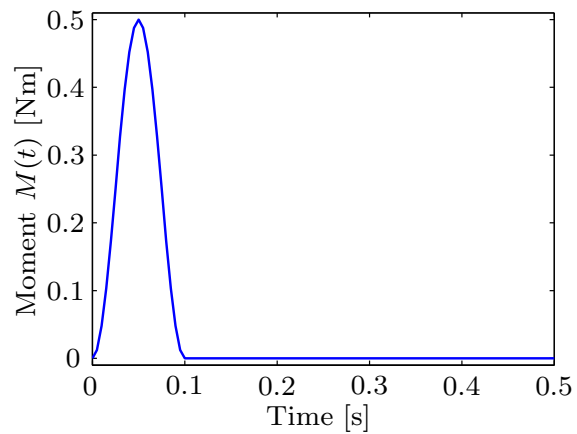

(a) Moment as function of time

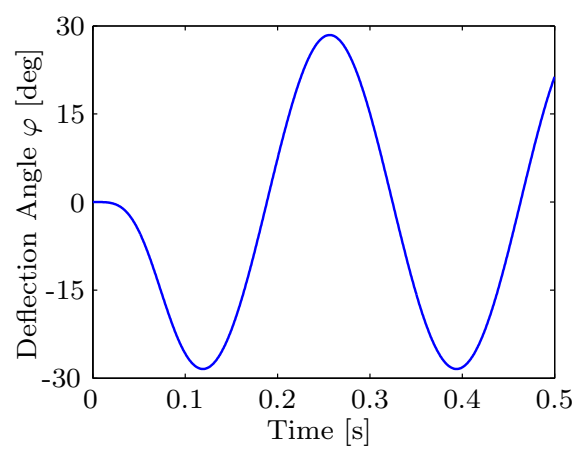

(b) Deflection angle as function of time

Figure 3.15: The moment $M(t)$ (a) and the resulting deflection angle $\varphi(\mathrm{b})$ as functions of time.

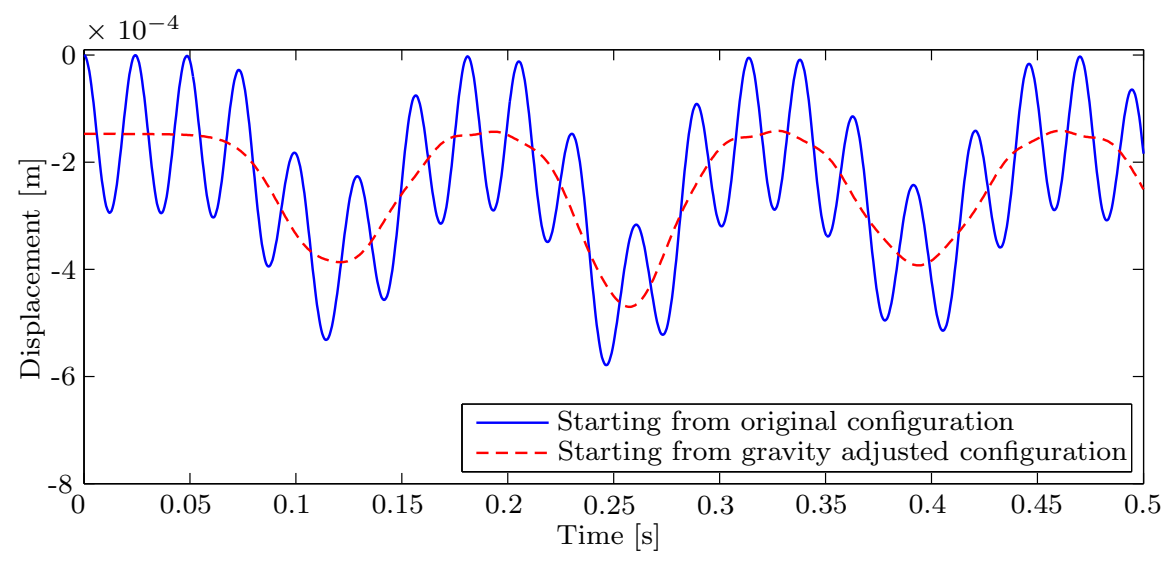

Figure 3.16: The $z$-displacement of node number 7 as a function of time for the simulations starting from the original configuration and the gravity adjusted equilibrium configuration.

Table 3.3: The static $z$-displacement of node number 7 at $\varphi=0, \varphi=-30$ and $\varphi=30$ degrees deflection.

\begin{tabular}{llll}
\hline & $\begin{array}{l}z \text {-displacement at } \\
\varphi=0 \text { degrees }[\mathrm{m}]\end{array}$ & $\begin{array}{l}z \text {-displacement at } \\
\varphi=-30 \text { degrees }[\mathrm{m}]\end{array}$ & $\begin{array}{l}z \text {-displacement at } \\
\varphi=30 \text { degrees }[\mathrm{m}]\end{array}$ \\
\hline & & & \\
FE-ANSYS & $-1.54 \cdot 10^{-4}$ & $-5.30 \cdot 10^{-4}$ & $-3.70 \cdot 10^{-4}$ \\
SE model & $-1.47 \cdot 10^{-4}$ & $-5.06 \cdot 10^{-4}$ & $-3.32 \cdot 10^{-4}$ \\
\hline
\end{tabular}


In Fig. 3.15(b) the deflection angle $\varphi$ is shown as a function of time. It is clear from this figure that the mechanism is undergoing a significant deflection of $\varphi= \pm 30$ degrees. In Fig. 3.16 the $z$-displacement of node number 7 is shown as a function of time. Due to the effects of gravity, an immediate oscillation of the $z$-displacement is observed for the simulation starting from the original undeformed configuration. This oscillation occurs with a frequency of about $40 \mathrm{~Hz}$ which corresponds to the second eigenfrequency of the model, see Fig. 3.14(a). It can be observed that the $z$-displacement is correctly oscillating around the results of the simulation starting from the gravity adjusted configuration. For the second time-response simulation, a periodic difference in $z$-displacement at e.g. around 0.12 and 0.26 seconds, can be observed. This is caused by the non-symmetrical layout of the mechanism. In Tab. 3.3 the same trend can be observed for the ANSYs FE model where we determined the static deflection due to gravity at $\varphi=0, \varphi=30$ and $\varphi=-30$ degrees deflection. The results of the superelement model agree qualitatively well with the ANSYS results.

\subsection{Conclusions}

A non-linear two-node superelement has been developed that is capable of modelling large rigid body motions and small elastic deformations of arbitrary-shaped flexible members with two interface surfaces. Absolute nodal coordinates are used to describe the configuration of the superelement with respect to an inertial reference frame. Furthermore, the formulation is based on a small rotation and displacement hypothesis in a local co-rotational frame. This allows for the application of reduction techniques, such as substructuring methods, for determining the equivalent mass and stiffness properties of the superelement from a linear finite element model. The reduced model is obtained by describing the nodal displacements with constraint, fixed-interface normal and deformable-interface modes. With the proposed deformable-interface modes, the deformations of the interface surfaces can be modelled. This allows for a compliant connection of superelements, provided that the elements at either side of the interface share compatible deformable-interface modes.

The use of deformable-interface modes in a large deflection analysis is demonstrated by considering a numerical example of a relative short compliant sheet flexure. For correct modelling of large deflections, a sufficient number of superelements have to be used such that the deformations of each individual superelement remain small with respect to a local co-rotational frame. The use of linear stiffness models for the computation of the stress resultants then remains valid. For this example, the correct modelling of the out-of-plane warping of the cross-section due to twist is of vital importance. A specific deformable-interface mode, referred to as a warping mode, is used for this purpose. It is demonstrated that due to the inclusion of the warping modes, correct dynamical results are obtained even for large deflections.

For the modelling of complex-shaped components that only exhibit small deflections, the fixed-interface normal modes of the Craig-Bampton method are 
used. It is demonstrated that only a few fixed-interface normal modes are required to accurately model the first few vibrational modes of a complex-shaped component.

In a third example, a compliant mechanism is considered which contains components of the first two examples. The vibrational modes of the superelement model are in good agreement over the full range of motion compared with results obtained using a classical non-linear finite element method. A time-response simulation of this mechanism is also provided. All the components of this mechanism are modelled with superelements, demonstrating the applicability of the approach.

Acknowledgements This research is financially supported by the Dutch association Point-One, project MOV-ET PNE08006, by the Dutch Department of Economic Affairs, Agriculture and Innovation. 



\section{Modelling a large-stroke compliant mechanism with superelements}

In this chapter, the superelement approach described in Chapters 2 and 3 is applied for the modelling of a two degree of freedom large-stroke compliant positioning mechanism. After first describing the background of the mechanism and its design, a SPACAR model consisting of non-linear beam and superelements is presented. Next, in order to verify the accuracy of this model, a FE model is developed using the commercially available FE software package Ansys. The chapter concludes with a comparison of the two models by an evaluation of eigenfrequencies along a prescribed path of the end-effector.

\subsection{Large-stroke compliant mechanism}

\subsubsection{Background}

There is a growing demand from industry for high precision positioning mechanisms which can be used in confined vacuum environments of e.g. lithography machines or electron microscopes. Conventional solutions, like stages based on roller bearings, suffer from hysteresis and wear and can contaminate the vacuum due to evaporation of lubricants. For an alternative solution, the use of compliant mechanisms that utilize the deflection of sheet and wire flexures to guide the motion, should be considered. Such mechanisms do not contaminate the vacuum, do not suffer from friction or backlash and have a low hysteresis [32, 65], allowing high-precision and repeatability. However, compliant mechanisms are traditionally only used for small-stroke applications, because flexures inherently lose stiffness in supporting directions when deflected (see Section 3.5.1). The stiffness in the support directions should ideally be as high as possible such that the resonance frequencies of unwanted vibrational modes are high, whereas the stiffness in the actuation directions should be as low as possible to allow for easy actuation of the mechanism. 


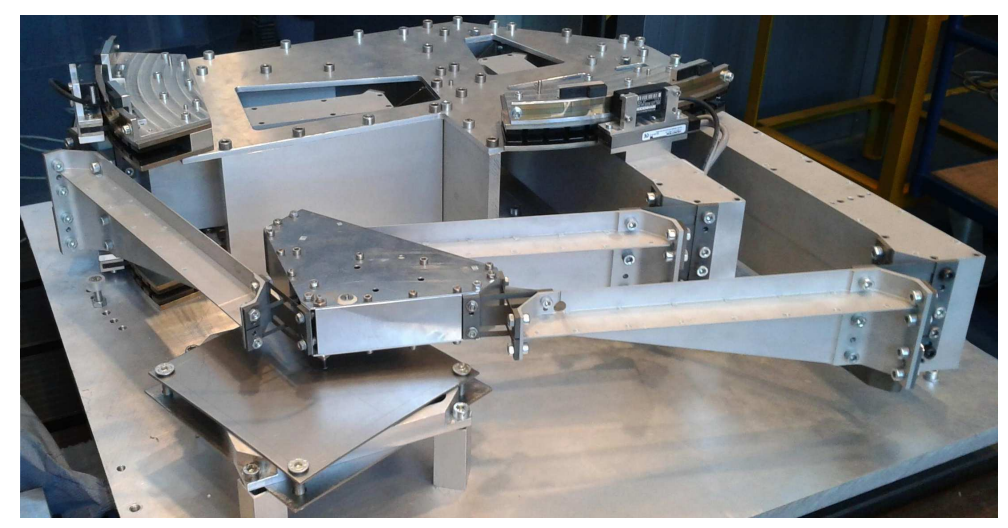

Figure 4.1: Photo of the two degree of freedom large-stroke compliant positioning mechanism.

The compliant mechanism shown in Fig. 4.1 is an attempt to obtain a design that combines these aspects of a high support stiffness and low actuation stiffness over a large-stroke planar motion. The mechanism is designed by Hoitzing [34] and Folkersma [27]. A lumped compliance approach [65] is used to achieve a large ratio of support over actuation stiffness [68]. This results in a design consisting of eleven compliant cross-hinges interconnected by stiff members. A cross-hinge consists of several sheet flexures that are arranged in such a way that the hinge has a low rotational stiffness, whereas all the other directions are designed to be stiff. The cross-hinges can undergo a significant deflection of \pm 20 degrees to realize a relatively large-stroke planar motion of the end-effector. The location of the cross-hinges and the lengths of the members are chosen such that the occupied space of the mechanism during operation is minimized for a certain work-space area.

To guarantee deterministic behaviour with the many assembled parts, the mechanism is designed to be exactly constrained [10, 16, 32, 65]. This means that no over- or underconstraints should be present in the design such that the system is kinematically and statically determinate. Exact constraint design, as opposed to elastic averaging, does not require tight tolerances on flatness, parallelism and squareness, and it allows for temperature fluctuations without excessive stresses in the structure. For compliant mechanisms that are not designed to be exactly constraint, overconstraining and miss-alignment can lead to load stiffening [7] and bifurcation [51]. Load stiffening negatively affects the actuation stiffness of the mechanism, whereas bifurcation can cause unstable behaviour and a sudden decrease in support stiffness. Furthermore, if underconstraints are present in the design, internal vibrational modes at relatively low frequencies can occur which can influence the closed-loop stability and precision of the mechanism.

To achieve an exact constraint design, local flexibilities are added to the members that are interconnecting the cross-hinges. Furthermore, the members are designed to be lightweight, which, in combination with the local flexibilities, gives a complex shape to their geometry. 
The mechanism has been built and eigenfrequency measurements are documented in $[27,70]$. Large differences between measurements and preliminary model results are observed, which are for the most part attributed to manufacturing and alignment errors, and damaged sheet flexures. Therefore, these experimental results are not repeated in this chapter.

\subsubsection{Mechanism details}

In Fig. 4.2 a drawing of the two degree of freedom large-stroke compliant positioning mechanism [27] is shown. Here, the complex-shaped flexible members that are interconnecting the cross-hinges are named: UpperArmX1, LowerArmX1, UpperArmX2, LowerArmX2, UpperArm Y, LowerArm Y and CrossBar. The motion of the mechanism is shown in Fig. 4.3, where the mechanism is simplified as a planar parallel kinematic mechanism with planar hinges interconnected by rigid members. Two parallelograms can be identified consisting of UpperArmX1, UpperArmX2 and CrossBar, and LowerArmX1, LowerArmX2, CrossBar and the end-effector, respectively. These two parallelograms constrain the rotation of the end-effector about the $z$-axis while allowing translations in the $x$ - and $y$-directions, respectively, hence the two degrees of freedom. The actuation is realized by two linear motors whose actuator coils are attached at locations above and below UpperArmX1 and UpperArm Y. The arm consisting of UpperArm $Y$ and LowerArm $Y$ allows both actuators to be placed at the base of the mechanism, avoiding the need to add the

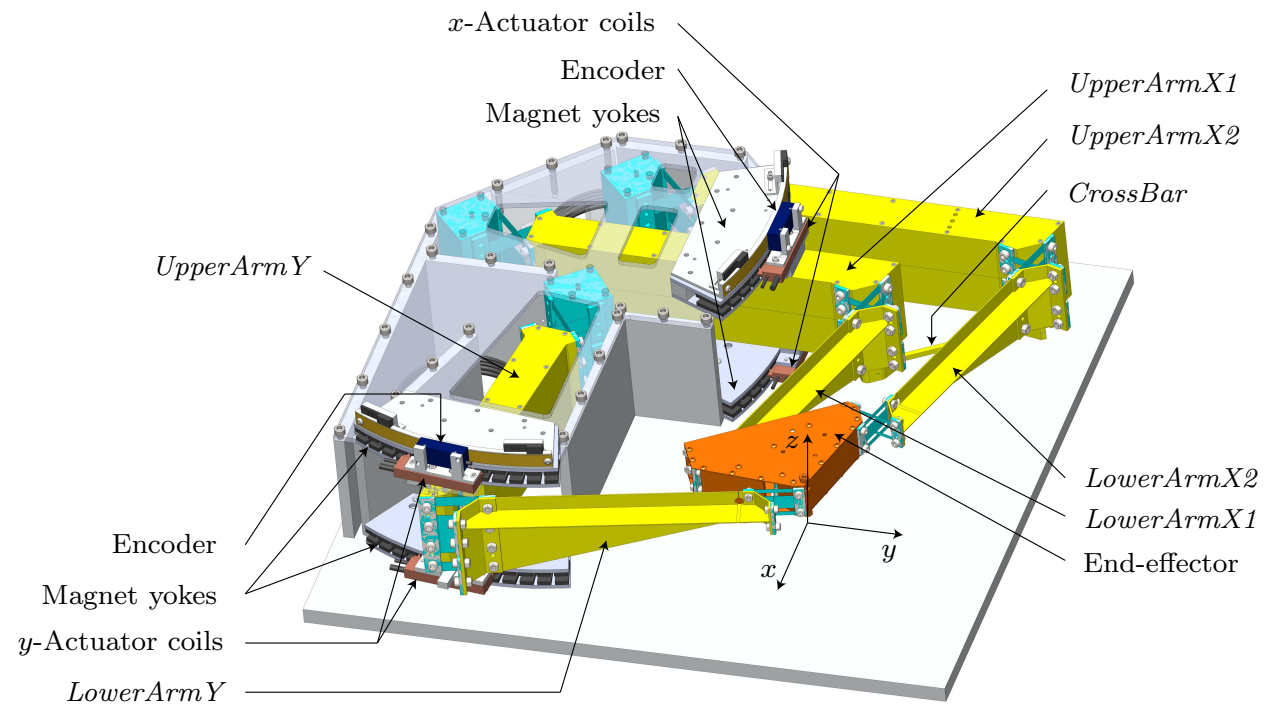

Figure 4.2: CAD drawing of the two degree of freedom large-stroke compliant positioning mechanism. 


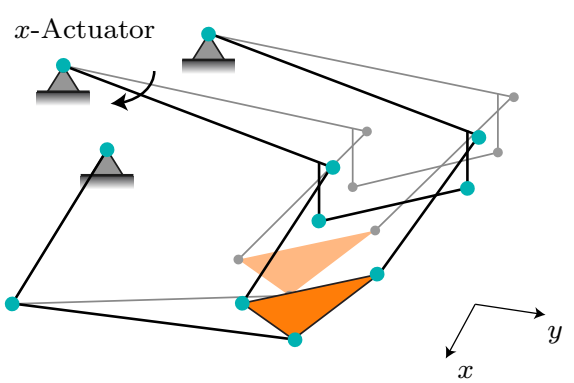

(a) Actuation in the $x$-direction

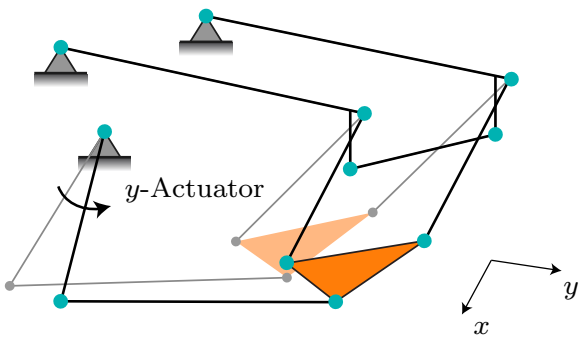

(b) Actuation in the $y$-direction

Figure 4.3: Schematic representation of the two degree of freedom largestroke compliant positioning mechanism, illustrating the planar $x$-directional motion (a) and the $y$-directional motion (b)

full weight of a magnet yoke to the moving mass. Instead, the magnet yokes can be attached to the support frame. Furthermore, two linear encoders are attached to UpperArm Y and LowerArm $Y$ for co-located feedback control. The size of the complete mechanism is $540 \times 585 \times 87 \mathrm{~mm}$ with a workspace area of $100 \times 100 \mathrm{~mm}$.

\subsubsection{Exact constraint design}

The compliant mechanism has been extensively analysed for over- and underconstraints in [17, 27], using the SVD approach proposed in [3]. Assuming that each cross-hinge only releases one rotation and that the interconnecting members are rigid, then each kinematic loop adds three overconstraints. The overconstraints are related to the direction perpendicular to the plane of motion. As the mechanisms has three such kinematic loops, two loops in the parallelograms and a third by the addition of the actuator arm UpperArm $Y$-LowerArm $Y$, it is nine times overconstrained. To release these overconstraints, the lower arms LowerArmX1, LowerArmX2 and LowerArm Y are designed to be compliant in the torsional and out-of-plane bending direction. Figure 4.4 shows that the torsion release is obtained by a T-profile shape and the bending release is implemented as a notch-hinge. As such, six of the overconstraints are released. The three remaining overconstraints are released in the CrossBar, see Fig. 4.5. Here the notch-hinges release the out-of-plane bending directions at either side of the CrossBar, whereas the wire and the sheet flexures allow for a torsional rotation of the component. Note that the CrossBar is now free to twist about its longitudinal axis, resulting in an internal vibration mode. However, from a FE analysis it is determined that the corresponding eigenfrequency is sufficiently high and should not disturb the dynamics of the end-effector or destabilize the control systems [17]. 


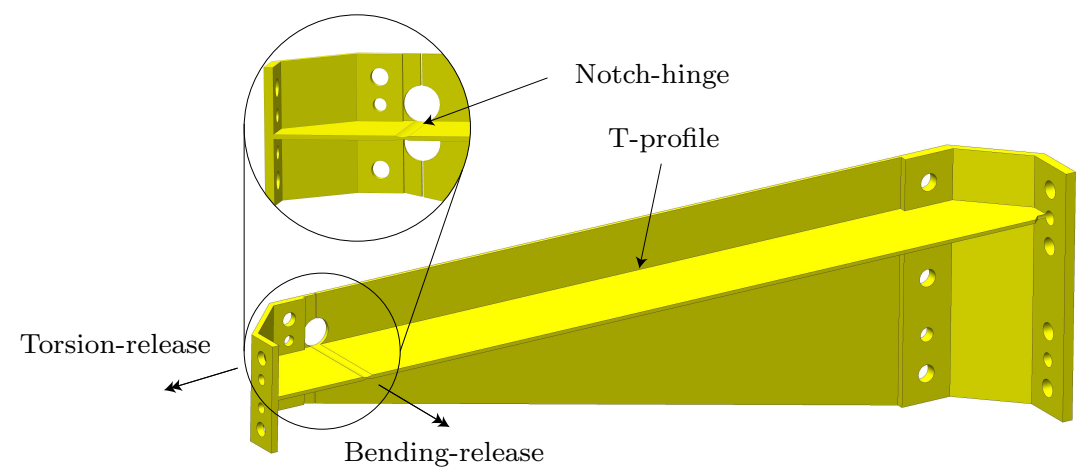

Figure 4.4: Illustration of LowerArmX1, showing the location of the bending and torsion releases.

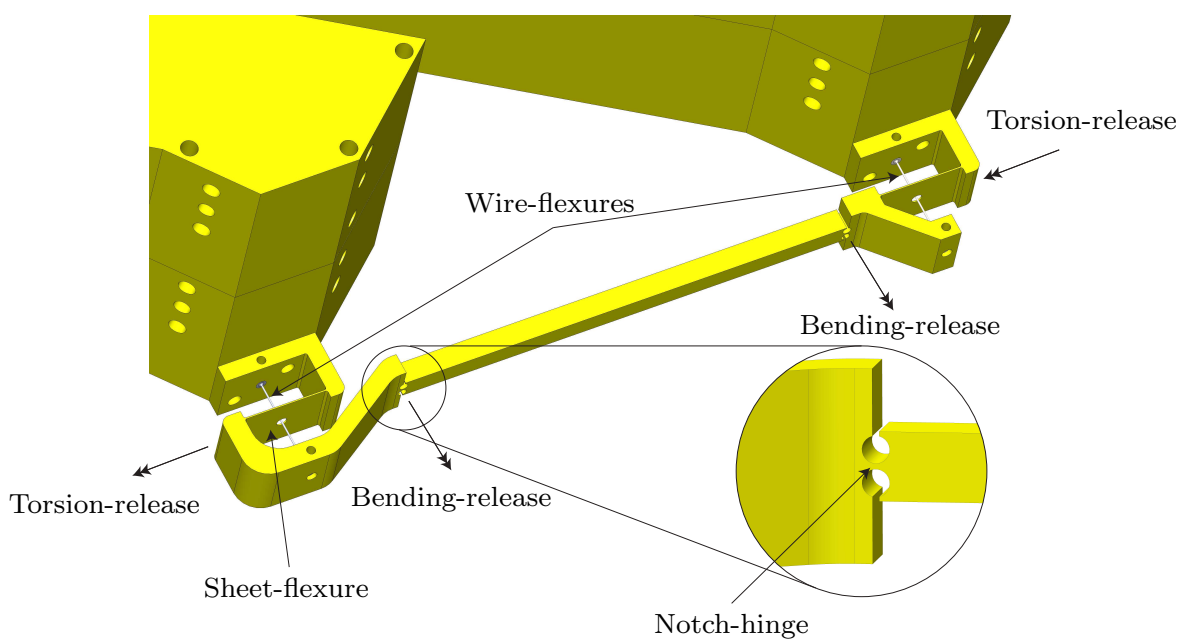

Figure 4.5: Illustration of CrossBar attached to UpperArmX1 and UpperArmX2, showing the location of the bending and torsion releases.

\subsection{Spacar mechanism model}

The complete SPACAR model of the compliant mechanism is shown in Fig. 4.6. It consists of 235 beam elements and 23 superelements. The assembly of these elements yields a model with 1584 nodal coordinates ${ }^{1}$ and 1822 deformation coordinates $^{2}$. Furthermore, the model has a total of 688 degrees of freedom. The

\footnotetext{
${ }^{1}$ The set of nodal coordinates consist of Cartesian coordinates, Euler parameters and warping coordinates associated with the superelement warping modes.

${ }^{2}$ The set of deformation coordinates are associated with the element deformation modes and the warping modes of the superelements.
} 


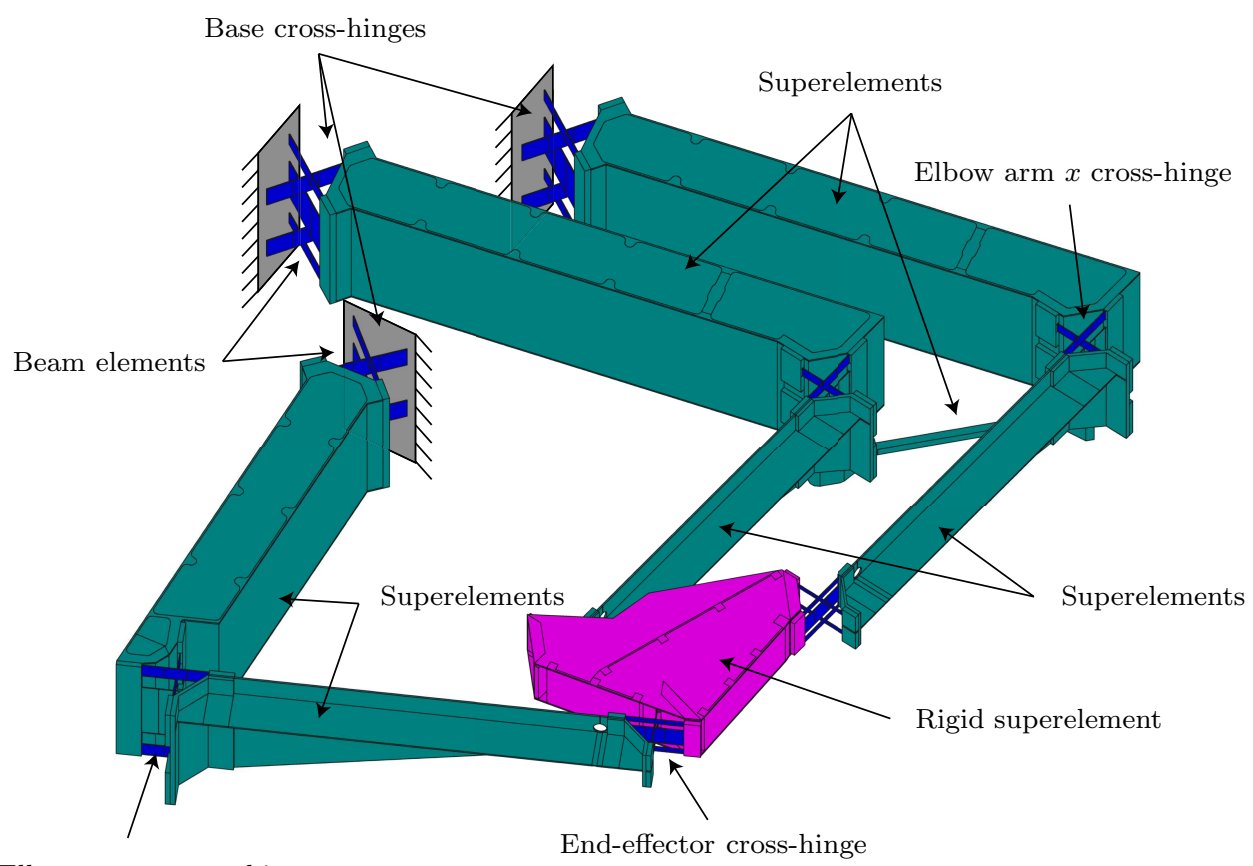

Elbow arm $y$ cross-hinge

Figure 4.6: SPACAR model of the two degree of freedom compliant mechanism.

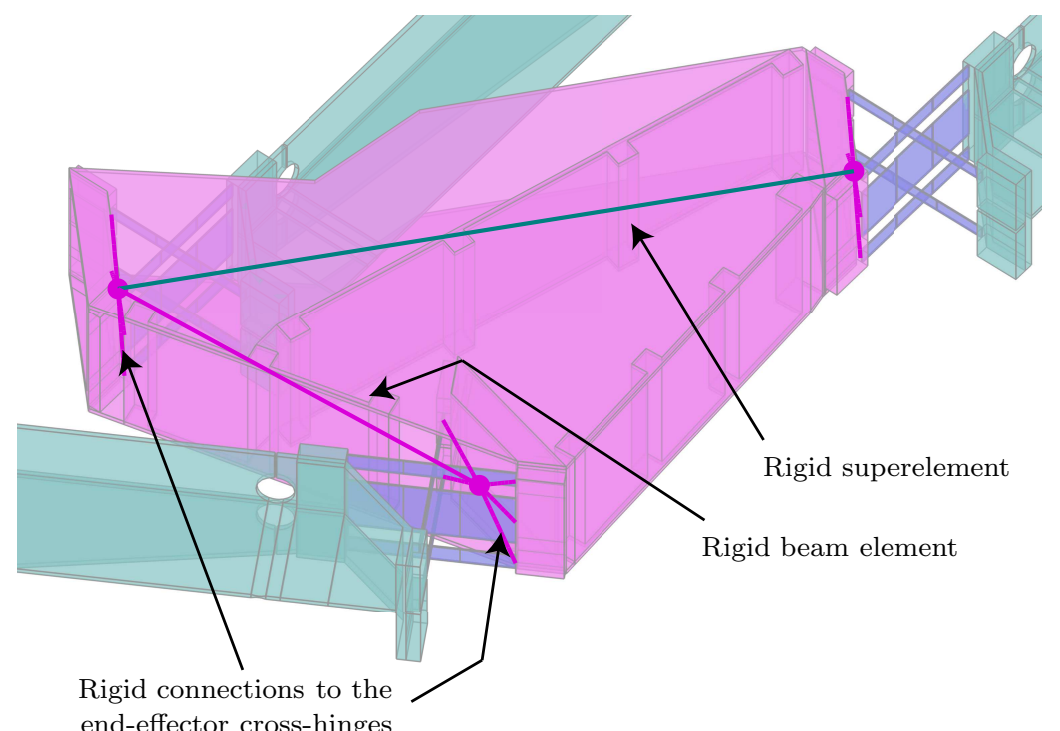

Figure 4.7: The end-effector modelled by a rigid superelement and a rigid beam element. 
complex-shaped flexible members are modelled with superelements while the compliant cross-hinges are modelled using both beam and superelements. The end-effector is considered to be rigid and modelled by a rigid superelement and a massless rigid beam element which are interconnecting the end-effector cross-hinges as is shown in Fig. 4.7. The end-effector inertia properties are included by the rigid superelement.

In the following subsections, the superelement modelling of the complex-shaped flexible members and the cross-hinge assemblies are discussed.

\subsubsection{Complex-shaped flexible members}

In Fig. 4.8 the FE model that is used for generating a superelement of UpperArmX1 is shown. It consists of 32316 20-node solid elements (ANSYs solid186). The body of the material is made of aluminum, whereas the flexure attachment parts are made of steel. The aluminum body is a thin-walled box with a wall thickness of $1 \mathrm{~mm}$. Steel screws are used to fix the flexure attachment parts to the aluminum body. The weight of the screws is taken into account by adding nodal point masses at their centres of mass. The FE model of UpperArmX1 is identical to UpperArmX2, except that for

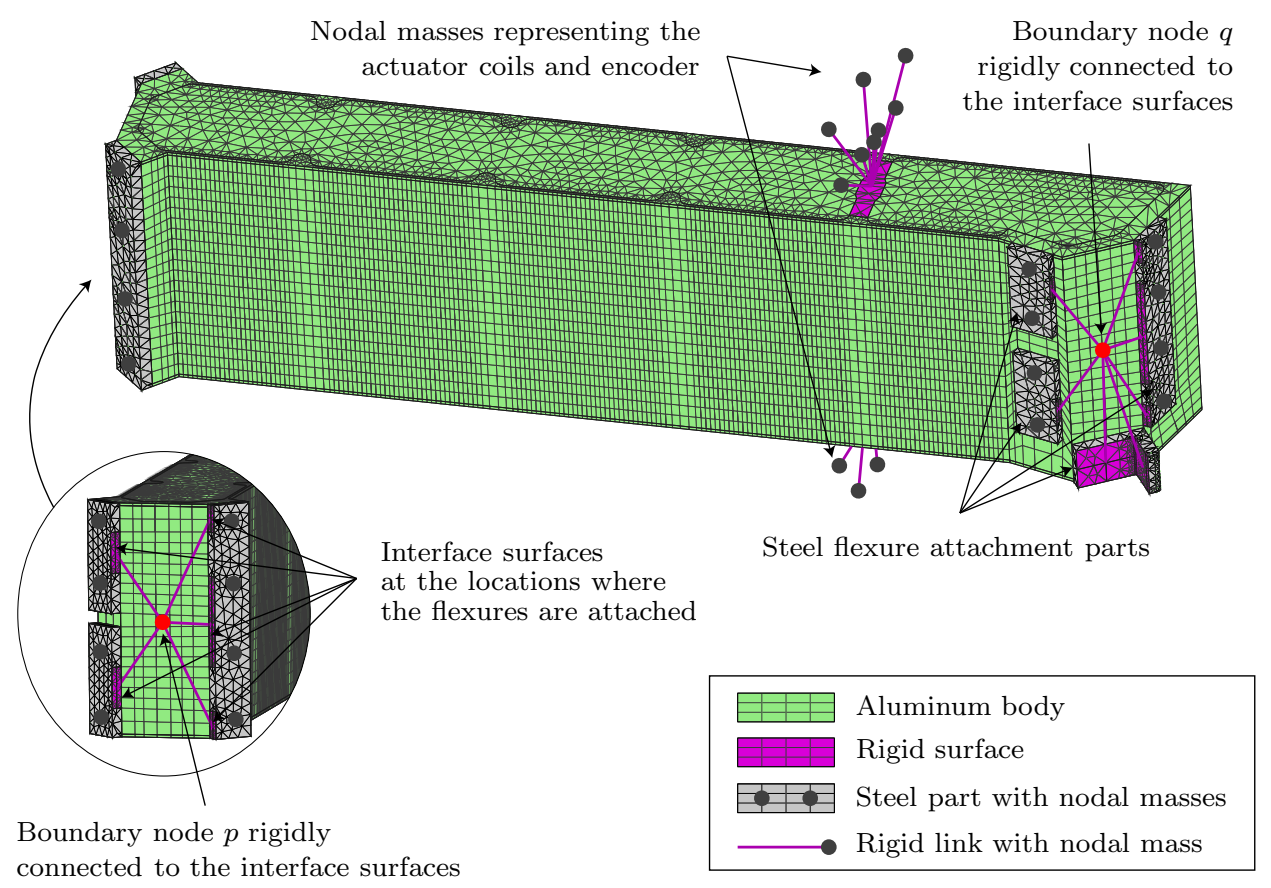

Figure 4.8: Finite element model of UpperArmX1, consisting of an aluminum body with steel flexure attachment parts and nodal point masses for representing the weight of the screws, the actuator coils and the encoder. For the generation of a superelement, boundary nodes are defined at either side of the component and are rigidly connected to the interface surfaces. 
Table 4.1: Number of solid elements used in the superelement FE models and the superelement lengths.

\begin{tabular}{lcc}
\hline Component name & Number of solid elements & Length [mm] \\
\hline UpperArmX1 & 32316 & 330.8 \\
UpperArmX2 & 32316 & 330.8 \\
UpperArm Y & 22702 & 240.9 \\
LowerArmX1 & 9893 & 232.1 \\
LowerArmX2 & 9893 & 232.1 \\
LowerArm Y & 11887 & 267.4 \\
CrossBar & 5736 & 153.9 \\
End-effector (rigid) & 13476 & 153.9
\end{tabular}

the former the weight of the $x$-actuator coils and the encoder should be included. For this purpose, the actuator-coils and encoder are modelled with point masses. Furthermore, point masses are placed at the mass centres of the components that are used to attach the actuator-coils and the encoder to the aluminum body. Rigid links are employed to connect these point masses to the FE model.

In a similar way, the FE models for the other complex-shaped flexible members are obtained. Table 4.1 shows on overview of the number of solid elements used in each of these models. Here, the UpperArm Y model includes the weight of an encoder and the $y$-actuator coils. Furthermore, the CrossBar is modelled using the physical properties of steel as it does not contain any aluminum parts.

To generate the superelements, interface surfaces and boundary node locations need to be defined. The interface surfaces correspond to the areas where the flexures of the cross-hinges are attached to the component. This is illustrated for UpperArmX1 in Fig. 4.8. The nodes on the interface surfaces at either side of UpperArmX1 are rigidly attached to their boundary nodes. The boundary node locations are chosen to be approximately at the average location of the interface surfaces. The distance between the boundary nodes corresponds to the length of the superelement, which are listed in Tab. 4.1.

The superelements of the complex-shaped flexible members are generated using only the constraint modes.

\subsubsection{Cross-hinge assemblies}

Two types of cross-hinges are used in the compliant mechanism. Attached to the CrossBar are cross-hinges consisting of a wire and a sheet flexure, whereas the other cross-hinges are assemblies of five sheet flexures. The modelling of the individual wire and sheet flexures is explained first. Then, the cross-hinge assemblies of the two types of cross-hinges are discussed.

\section{Wire and sheet modelling}

All the wire and sheet flexures of the cross-hinges are made of steel and can undergo significant deflection of \pm 20 degrees during the motion of the mechanism. The wire 
flexures are thin and slender components. Therefore, beam elements are well suited to model these components. Each wire flexure is modelled using three beam elements, such that the large deflections are modelled correctly.

The sheet flexures are modelled using three beam elements or superelements. Both ends of the sheet flexures are considered to be rigidly clamped to the complex-shaped flexible members. Most of the sheet flexures in the model are slender, i.e. their height to length ratio is relatively small. Then, beam elements can be used to model the sheet flexures. If the height to length ratio is large, then the warping of the cross-section needs to be modelled and suppressed at both ends of the sheet flexure to correctly model the rigid clamping as is demonstrated in Section 3.5.1. This is achieved by using superelements with additional warping modes.

\section{Cross-hinges attached to the crossBar}

In Fig. 4.9 the SPACAR model is shown of the subassembly where the CrossBar is attached to the cross-hinges that consist of wire and sheet flexures (see Fig. 4.5). In Tab. 4.2 the dimensions of the wire and sheet flexures are given. The wire flexures are modelled with beam elements as is previously mentioned. Superelements are used to model the sheet flexures due to their relatively large height to length ratio (0.7). Rigid beam elements are used to interconnect the wire and sheet flexures and to facilitate the connection with the CrossBar. Rigid beams are also employed to connect this subassembly with the boundary nodes of UpperArmX1 and UpperArmX2 as is shown in Fig. 4.10.

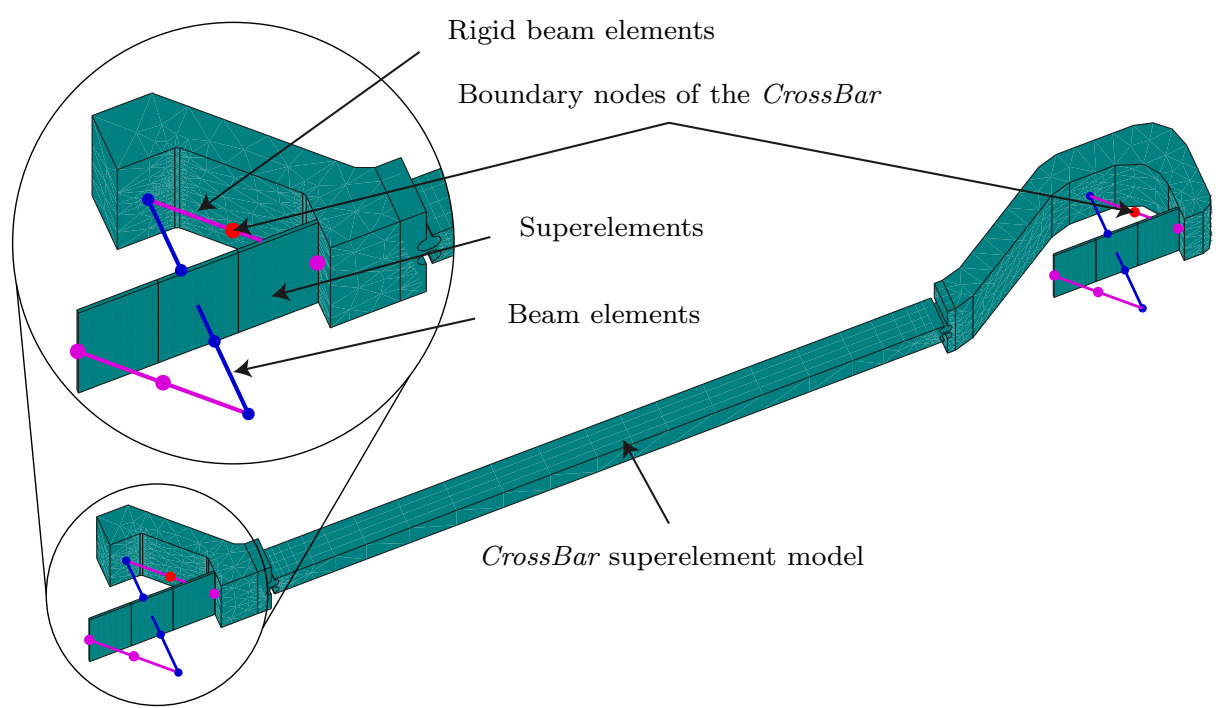

Figure 4.9: SPACAR model of the CrossBar attached to the wire-sheet flexure hinges. 


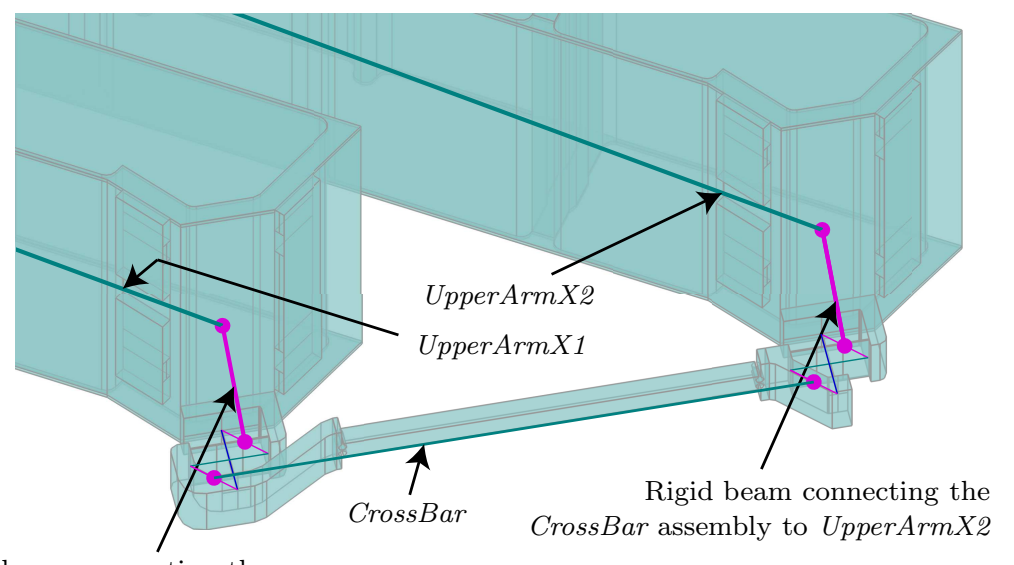

Rigid beam connecting the

CrossBar assembly to UpperArmX1

Figure 4.10: Rigid connection between the CrossBar and UpperArmX1 and between the CrossBar and UpperArmX2.

Table 4.2: Flexure dimensions of the wire-sheet flexure hinge.

\begin{tabular}{lc}
\hline & Dimensions $[\mathrm{mm}]$ \\
\hline Sheet flexure length & 20 \\
Sheet flexure height & 14 \\
Sheet flexure thickness & 0.3 \\
Wire flexure length & 20 \\
Wire flexure diameter & 0.4
\end{tabular}

\section{Cross-hinge composed of five sheet flexures}

Figure 4.6 shows the locations of the different types of cross-hinges composed of five sheet flexures which are referred to as: base cross-hinge, elbow arm $x$ cross-hinge, elbow arm $y$ cross-hinge and end-effector cross-hinge, respectively. In Fig. 4.11, the SPACAR model of a base cross-hinge is depicted and in Tab. 4.3 an overview of the flexure dimensions for the different cross-hinges is given. For the base and end-effector cross-hinges, the main flexure, the two outer flexures and the two cross flexures, are all modelled with three beam elements. Three superelements are used to model the main flexures of the elbow cross-hinges due to their relatively large height to length ratio. At either side of the cross-hinge, the flexures are interconnected with rigid beam elements to the attachment nodes of the cross-hinge subassembly, see Fig. 4.11. In turn, the complex-shaped flexible members are attached to the cross-hinge assemblies by use of these attachment nodes, yielding the complete SPACAR model of the compliant mechanism ${ }^{3}$.

\footnotetext{
${ }^{3}$ See Appendix D for the equations of motion of a multibody system with superelements.
} 


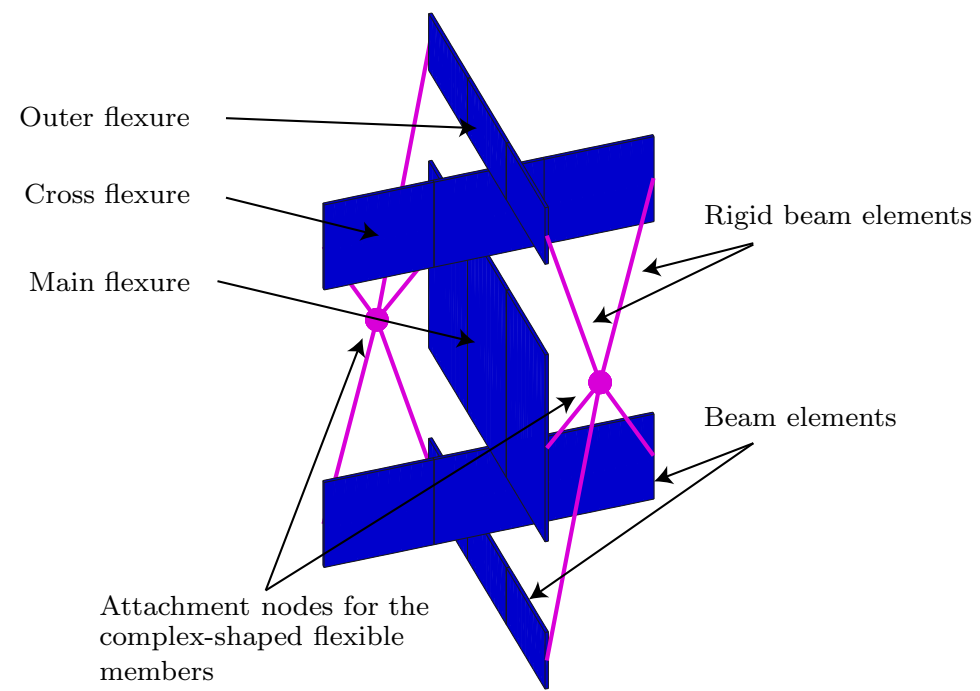

Figure 4.11: SPACAR model of one of the base cross-hinges.

Table 4.3: Flexure dimensions of the cross-hinges.

\begin{tabular}{lcccc}
\hline & \multicolumn{4}{c}{ Cross-hinge locations } \\
& Base & Elbow arm- $x$ & Elbow arm- $y$ & End-effector \\
\hline Flexure length [mm] & 50 & 40 & 40 & 35 \\
Flexure thickness [mm] & 0.5 & 0.4 & 0.4 & 0.3 \\
Main flexure height [mm] & 33 & 38 & 43 & 14 \\
Outer flexure height [mm] & 10 & 8 & 10 & 4 \\
Cross flexure height [mm] & 15 & 7 & 10 & 3
\end{tabular}

\subsection{Ansys mechanism model}

The complex-shaped flexible members in the FE model are modelled with 20-node solid elements. The meshes of the complex-shaped flexible members are identical to the meshes of the superelements of the SPACAR model in Section 4.2, including the nodal point masses for modelling the screws, actuator coils and encoders. The wire and sheet flexures are modelled with beam elements (ANSYs beam188) and 8-node shell elements (ANSYS shell281), respectively, in order to reduce the size of the FE model without losing accuracy.

The connection between the solid, shell and beam elements requires some attention as the solid elements only have translational degrees of freedom, whereas the shell and beam elements have both translational and rotational degrees of freedom. Two different approaches are considered to properly connect these elements. In the 


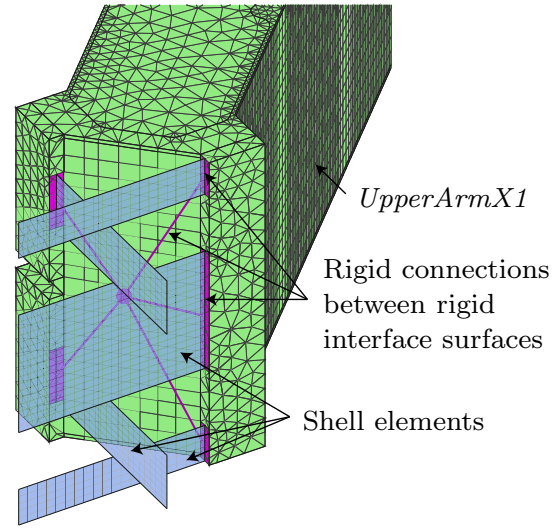

(a) Interface surfaces rigidly interconnected

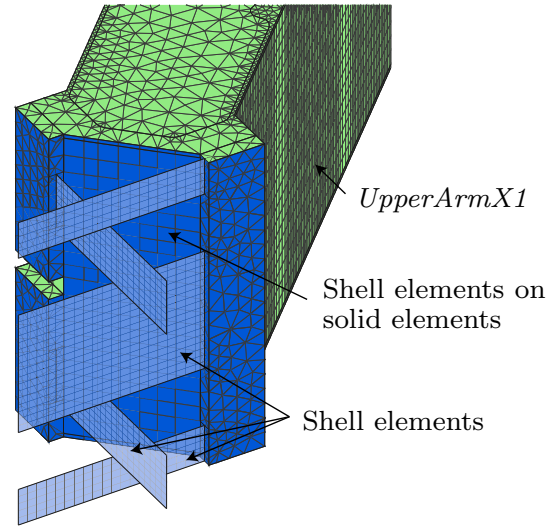

(b) Massless shell elements overlaid on solid elements

Figure 4.12: Two approaches for interconnecting shell and solid elements.

first approach, the interface surfaces of the complex-shaped flexible members are rigidly interconnected by multipoint constraint elements (ANSYS mpc184), which is consistent with the SPACAR model. This is illustrated in Fig. 4.12(a) for connecting the shell elements of the base cross-hinge flexures with the solid elements of the UpperArmX1 model. A more realistic connection can be obtained by overlaying massless shell elements on the solid elements to which the flexures are attached. The shell elements of the flexures are then connected to the massless shell elements, see Fig. 4.12(a). This allows for an elastic connection between the shell and solid elements. The two models are respectively referred to by FE Ansys rigid connection and FE Ansys shell connection.

The complete FE model of the compliant mechanism consists of 13821920 node solid elements, 6232 8-node shell elements (ANsys shell281) and 6 beam elements (ANsYs beam188). The model has approximately 1.5 million degrees of freedom.

\subsection{Comparison of eigenfrequencies}

To evaluate the accuracy of the SPACAR model, eigenfrequency results up to $200 \mathrm{~Hz}$ are obtained along a prescribed path of the end-effector and are compared to those obtained with the ANSYS models. In Fig. 4.13 the prescribed path of the end-effector is shown, where the coordinate $s$ is the distance travelled along the path in the indicated direction. The first section of the path is defined by a third order vector polynomial,

$$
\mathcal{P}_{1}(\xi)=\left(\xi-2 \xi^{2}+\xi^{3}\right)\left[\begin{array}{l}
r \\
0
\end{array}\right]+\left(3 \xi^{2}-2 \xi^{3}\right)\left[\begin{array}{l}
r \\
0
\end{array}\right]+\left(\xi^{3}-\xi^{2}\right)\left[\begin{array}{l}
0 \\
r
\end{array}\right],
$$




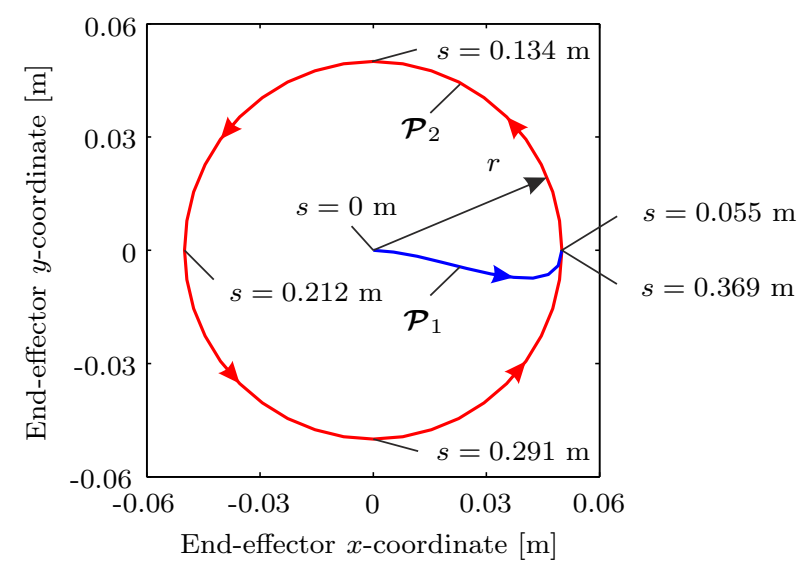

Figure 4.13: Prescribed path of the end-effector.

whereas the second part is a circular motion described by,

$$
\mathcal{P}_{2}(\xi)=\left[\begin{array}{c}
r \cos (2 \pi \xi) \\
r \sin (2 \pi \xi)
\end{array}\right],
$$

with

$$
0 \leq \xi \leq 1
$$

for both parts of the path. Here, the vector functions $\mathcal{P}_{1}$ and $\mathcal{P}_{2}$ describe the $x$ - and $y$-coordinates of the path with the radius $r=0.05 \mathrm{~m}$.

For the SPACAR and ANSYS models, eigenfrequency results are obtained in 50 equilibrium configurations along the path. For the SPACAR model, the eigenfrequencies are computed by solving the generalized eigenvalue problem,

$$
\left(\boldsymbol{K}_{\boldsymbol{q}}^{(i)}+\boldsymbol{G}_{\boldsymbol{q}}^{(i)}-\omega_{j}^{(i) 2} \boldsymbol{M}_{\boldsymbol{q}}^{(i)}\right) \boldsymbol{v}_{j}^{(i)}=\mathbf{0} \quad \text { with } \quad j=1,2, \ldots, k,
$$

where $\boldsymbol{K}_{\boldsymbol{q}}$ and $\boldsymbol{G}_{\boldsymbol{q}}$ are the structural and geometric stiffness matrices, $\boldsymbol{M}_{\boldsymbol{q}}$ is the mass matrix and $\boldsymbol{v}_{j}$ and $\omega_{j}$ correspond to the $j^{\text {th }}$ eigenvector and eigenfrequency, respectively. Furthermore, $k$ are the number of degrees of freedom of the model. The matrices $\boldsymbol{K}_{\boldsymbol{q}}, \boldsymbol{G}_{\boldsymbol{q}}$ and $\boldsymbol{M}_{\boldsymbol{q}}$ are obtained by linearizing the equations of motion in the $i^{\text {th }}$ configuration along the path, see Appendix E. The geometric stiffness matrix $\boldsymbol{G}_{\boldsymbol{q}}$ describes stress-stiffening effects and only has a small influence on the first two eigenfrequencies and negligible influence on the higher eigenfrequencies, in this case. For the ANSYS models, stress-stiffening effects are also included.

It took approximately two hours of computation to obtain the eigenfrequency results along the path for the SPACAR model with 688 degrees of freedom on a $2.20 \mathrm{GHz}$ computer with $8 \mathrm{~GB}$ of memory. In contrast for the ANSYS models with approximately 
1.5 million degrees of freedom, results were obtained after two days of computing on a faster $3.40 \mathrm{GHz}$ computer with $32 \mathrm{~GB}$ of memory.

In Fig. 4.14 the first two eigenfrequencies as functions of the distance travelled along the path are shown. The corresponding vibrational modes are the actuator modes of the mechanism, which are illustrated in Fig. 4.15 for the initial configuration. The first two eigenfrequencies remain fairly constant along the path. Furthermore, it can be observed that the first two eigenfrequencies of the three models are quite close together.

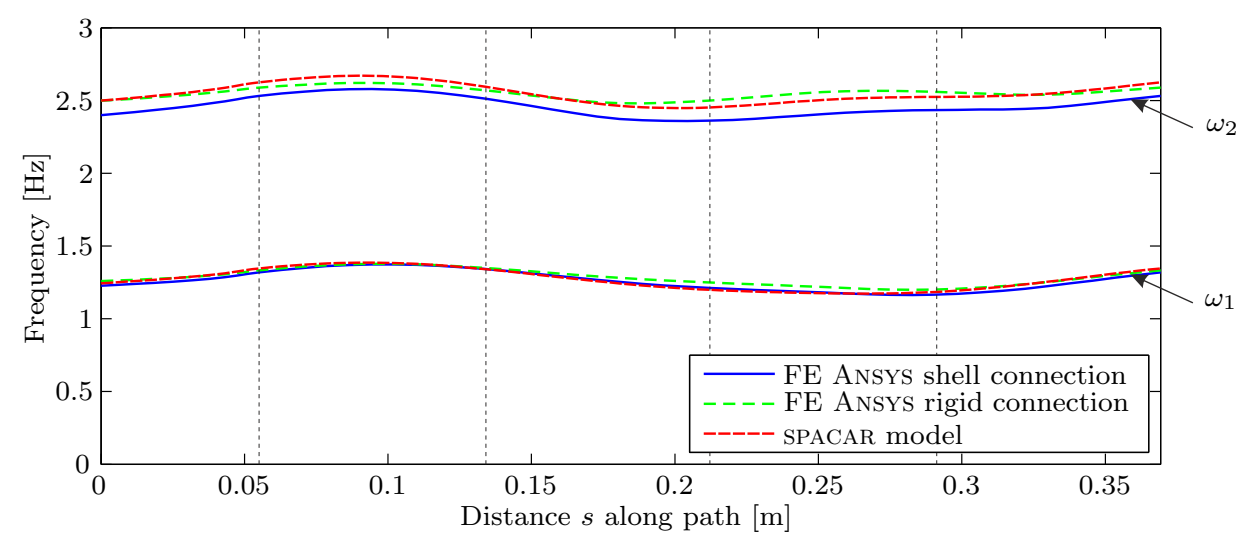

Figure 4.14: The first two eigenfrequencies $\omega_{1}$ and $\omega_{2}$ of the two degree of freedom large-stroke compliant mechanism as functions of the distance along the path. The vertical dashed lines correspond to the locations marked in Fig. 4.13 .

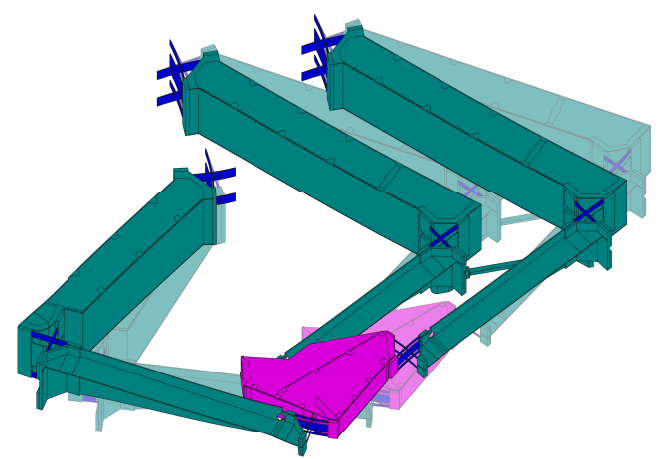

(a) First vibrational mode

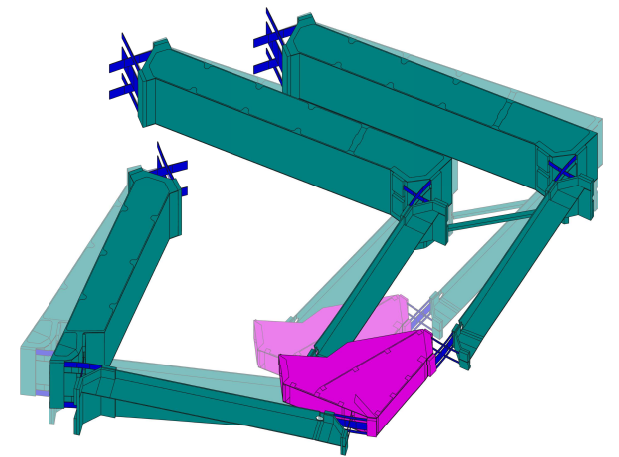

(b) Second vibrational mode

Figure 4.15: The first two vibrational modes of the two degree of freedom large-stroke compliant mechanism in the undeflected configuration. 


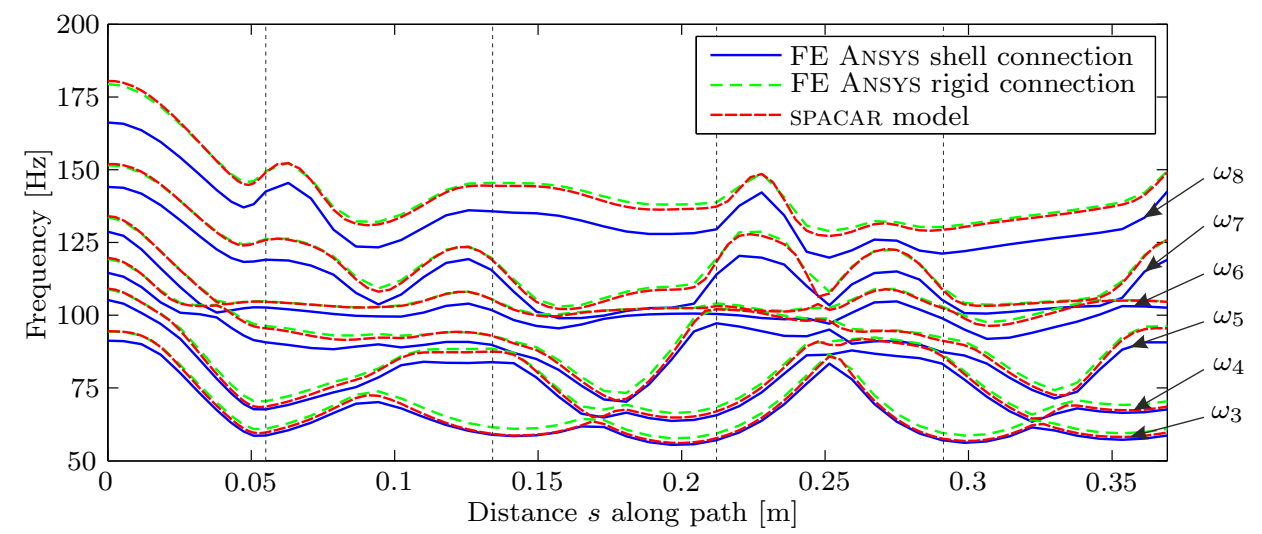

Figure 4.16: Eigenfrequencies $\omega_{3}$ through $\omega_{8}$ of the two degree of freedom large-stroke compliant mechanism as functions of the distance along the path. The vertical dashed lines correspond to the locations marked in Fig. 4.13.

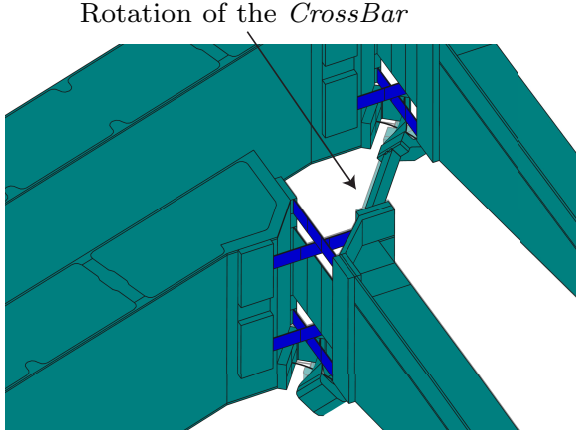

(a) Third vibrational mode

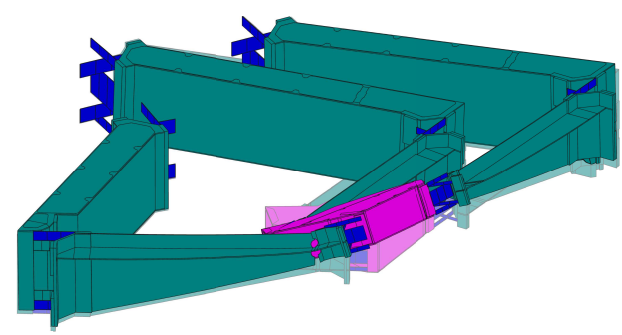

(b) Fourth vibrational mode

Figure 4.17: The third and fourth vibrational modes of the two degree of freedom large-stroke compliant mechanism in the undeflected configuration.

Figure 4.16 shows the third up to the eighth eigenfrequencies as functions of the path distance. Here, the third eigenfrequency corresponds to the internal vibrational mode of the CrossBar, as is shown in Fig. 4.17(a). Furthermore, the fourth eigenfrequency corresponds to the first out-of-plane mode shown in Fig. 4.17(b). It can be observed that initially the eigenfrequencies are quickly declining as the mechanism is deflected. This decline is caused by the loss of stiffness in the supporting directions of the flexures when they are deflected. The results of the SPACAR model and the ANSYS model with the rigid connections are very close together. Up to the sixth eigenfrequency, all three models produce similar results, 
although the SPACAR model and the ANSYS model with the rigid connections produce slightly higher eigenfrequencies than the ANSYs model with the more realistic shell connections. This effect is more pronounced for the seventh and eighth eigenfrequencies.

\subsection{Conclusions}

A SPACAR model of a two degree of freedom large-stroke compliant mechanism has been developed. The mechanism consists of eleven cross-hinges interconnected by seven complex-shaped flexible members and an end-effector. The cross-hinges are modelled with beam elements and superelements. Furthermore, the complex-shaped flexible members are modelled with superelements, as is the end-effector which is considered to be rigid.

To verify the validity of the SPACAR model, eigenfrequency results are computed along a prescribed path and are compared to results obtained with two ANSYS models. One of the ANSYs models includes, similar to the SPACAR model, rigid connections between the cross-hinges and the complex-shaped flexible members. The eigenfrequency results of the SPACAR model and this ANSYS model are very close together over the full range of motion. The results obtained with the other, more realistic, ANSYS model are in good agreement with the SPACAR model up to the sixth eigenfrequency. The seventh and eighth eigenfrequencies are slightly higher for the SPACAR model.

The accuracy of the SPACAR model could be further improved if the rigid connections between the cross-hinges and the complex-shaped flexible members are modelled deformable. This can be achieved by replacing the beam elements in the cross-hinges with superelements having deformable-interface modes that correctly model the deformability at the interconnections between the superelement models. Nevertheless, for purposes such as closed-loop time-response simulations, the developed SPACAR model is considered to be sufficiently accurate.

The SPACAR model has 688 degrees of freedom which is significantly less than the 1.5 million degrees of freedom of the ANsYs models. This allows results to be obtained within hours for the SPACAR model, instead of days for the ANSYS models. However, the SPACAR model is still too complex for reasonably fast time-response simulations. This requires further model reduction of the SPACAR model as will be discussed in the following chapters. 
Part II

System model reduction 



\title{
Model reduction for efficient time-integration of planar flexible multibody models
}

\begin{abstract}
A reduction method is proposed for efficient time-integration of (planar) compliant mechanism models that undergo large deflections. Models for this class of mechanisms have to include an accurate description of geometric non-linearities, as stiffness characteristics can change significantly during deflection. A finite element based flexible multibody approach is used to analyse compliant mechanisms in terms of independent generalized coordinates. Geometric transfer functions are applied to express the configuration and deformed state in terms of these independent coordinates. The modelling of large deflections requires using a sufficient number of finite elements to ensure that deformations remain small in a co-rotational context. Increasing the number of elements, increases, besides the number of degrees of freedom, the largest eigenfrequency in the model. This reduces the allowable step size in explicit time-integrator methods. For the proposed reduction method, we aim to suppress the unwanted high frequency modes to increase the allowable step size. This is accomplished by first choosing, where possible, coordinates that remain small during simulation as independent coordinates. Such coordinates are well suited to be reduced using linear projection methods such as modal projection for suppressing the high frequency modes. The configuration and deformed state of the mechanism is subsequently determined with the geometric transfer functions. Consequently, a significant increase in the allowable step size is realized, while retaining the geometric non-linear effects that are contained in the geometric transfer functions. The effectiveness of the method is demonstrated by two planar examples: a compliant straight guidance that undergoes large deflections and a flexible manipulator.
\end{abstract}

Published in: International Journal of Non-Linear Mechanics (S.E. Boer, D. ten Hoopen, R.G.K.M. Aarts, W.B.J. Hakvoort, J.B. Jonker) 


\section{$5.1 \quad$ Introduction}

Compliant mechanisms equipped with flexure based hinges are often utilized in high precision manipulator devices for their deterministic static and dynamic behaviour. The analysis for design and control of compliant mechanisms undergoing large deflections, relies on computational efficient models that are capable of capturing the relevant dynamic and compliant characteristics over the full range of motion. These models should offer a sound inclusion of geometric non-linear effects.

In [2] we discuss a modelling approach for the analysis and simulation of multibody systems with flexible components where a multibody system is modelled as the assembly of non-linear finite elements. For each element a fixed number of deformation modes are defined with associated deformation coordinates that are invariant for arbitrary rigid body motion. Typical flexible members of compliant mechanisms are wire and sheets flexures. These flexures may be considered as one-dimensional structures which can be correctly modelled by flexible beam elements. Though, a rather large number of flexible beam elements may be needed to obtain a model that correctly captures the geometric non-linear effects for large deformations. Consequently, high frequency modes are introduced in the model that greatly deteriorate the computational efficiency, in particular if explicit integrators are used for the time-integration of the non-linear equations of motion.

For linear mechanical systems, these higher frequency modes can be removed by using model reduction techniques that project the dynamics onto a lower dimensional subspace while preserving the dominant dynamical behaviour. Research on model reduction techniques applied to non-linear flexible multibody systems can be separated into two categories: component and system level model reduction. System level model reduction generally has a large impact on the computational time of simulations, whereas component level reduction techniques are employed for the efficient modelling of complex-shaped components [11, 21, 48, 59]. The adaptive modal integration (AMI) method introduced by Aarts and Jonker [1] and the global modal parameterization (GMP) method introduced by Brüls et al. [18] are examples of system level model reduction techniques. Both methods separate the motion of the mechanism in a non-linear nominal rigid body motion and a configuration dependent elastic motion part. The AMI method is applied to a set of non-linear equations of motion expressed as ordinary differential equations (ODE) for a set of independent coordinates, as in [2]. By using locally linearized models, a set of linear time-varying ODE's is obtained for the elastic motion. With modal reduction techniques, the order of these equations is reduced. The GMP method is applied to a general set of differential algebraic equations (DAE). By combining linearized models with the constraint equations and applying modal reduction techniques, also a cheaper to solve set of ODE's is obtained for the elastic motion. Both methods in their current approach assume that the elastic deformations remain small with respect to the rigid body motion, making them less suited to reduce models describing compliant mechanisms that undergo large deflections.

For this reason, we aim to reduce the non-linear equations of motion of a multibody system with flexible components expressed as ODE's for a set of 
independent coordinates [2]. This approach has been implemented in the SPACAR software [39]. Geometric transfer functions are used to express the configuration and deformation state of the mechanism as explicit functions of these independent coordinates. In the proposed reduction method, the number of independent coordinates is reduced by imposing linear relations between them. To that end independent coordinates are selected which remain small during simulation and are therefore suitable to be reduced using linear projection methods. The reduction of independent coordinates effectively suppresses the excitation of high frequency modes while the dominant geometric non-linear effects are retained in the geometric transfer functions. This makes the approach suitable for reduction of compliant mechanisms undergoing large deflections.

The effectiveness of the method is demonstrated with two planar examples: a compliant straight guidance that undergoes large deflections and a flexible manipulator. The applicability of the reduction method is shown for these classes of planar mechanisms, whereas spatial systems are addressed elsewhere [13] (Chapter 6).

\section{$5.2 \quad$ Finite element modelling}

A brief introduction is given on the finite element representation used to model the compliant mechanism and flexible manipulator considered in this paper. Then, using the concept of geometric transfer functions, we derive the equations of motion in terms of independent generalized coordinates. Finally, the linearized equations of motion are presented which are used for modal analyses in Section 5.3.

\subsubsection{Finite element representation}

In the presented finite element method, a compliant mechanism or a flexible manipulator is modelled as an assembly of finite elements interconnected by joint elements which can be hinge elements or a subassembly of flexible beam elements that represent a flexible joint. The location of each element is described relative to a fixed inertial coordinate system by a set of nodal coordinates $\boldsymbol{x}^{(k)}$, valid for large displacements and rotations. Some coordinates are Cartesian coordinates of the end nodes, while others describe the orientation of orthogonal base vectors or triads, rigidly attached to the element nodes. The superscript $k$ indicates that a specific element $k$ is considered. With respect to some reference configuration of the element, the instantaneous values of the nodal coordinates represent a fixed number of deformation modes for the element. The deformation modes are specified by a set of deformation coordinates $\varepsilon^{(k)}$ that are invariant for arbitrary motion of the element as a rigid body. Hence, the number of deformation coordinates is equal to the number of nodal coordinates minus the number of degrees of freedom of the element as a rigid body. The components of the vector of deformation coordinates $\varepsilon^{(k)}$ can be expressed as analytical functions of the vector of nodal coordinates $\boldsymbol{x}^{(k)}$. In this way we can define for each element $k$ a vector function

$$
\boldsymbol{\varepsilon}^{(k)}=\mathcal{D}^{(k)}\left(\boldsymbol{x}^{(k)}\right) .
$$


As a first example, the deformation functions $\mathcal{D}_{i}^{(k)}$ of the planar beam element are presented. As nodal coordinates we have four Cartesian coordinates $\left(x^{p}, y^{p}\right)$, $\left(x^{q}, y^{q}\right)$ describing the nodal positions of the beam element in the inertial reference frame and two rotation angles $\varphi^{p}$ and $\varphi^{q}$ that describe the orientations of the nodes with respect to the initial undeformed orientation of the element given by the angle $\beta_{0}$. Hence the vector of nodal coordinates is then

$$
\boldsymbol{x}_{\text {beam }}^{(k)}=\left[\begin{array}{c}
\boldsymbol{r}^{p} \\
\varphi^{p} \\
\boldsymbol{r}^{q} \\
\varphi^{q}
\end{array}\right]=\left[\begin{array}{lllllll}
x^{p} & y^{p} & \varphi^{p} & \mid & x^{q} & y^{q} & \varphi^{q}
\end{array}\right]^{\mathrm{T}} .
$$

The number of degrees of freedom of the element as a rigid body is three, which gives rise to three deformation coordinates. The first deformation coordinate $\varepsilon_{1}^{(k)}$ represents the elongation of the element

$$
\begin{aligned}
\varepsilon_{1}^{(k)} & =\mathcal{D}_{1}^{(k)}\left(\boldsymbol{x}_{\text {beam }}^{(k)}\right) \\
& =l^{(k)}-l_{0}^{(k)}+\frac{1}{30 l_{0}^{(k)}}\left(2\left(\varepsilon_{2}^{(k)}\right)^{2}+\varepsilon_{2}^{(k)} \varepsilon_{3}^{(k)}+2\left(\varepsilon_{3}^{(k)}\right)^{2}\right),
\end{aligned}
$$

where $l^{(k)}=\left\|\boldsymbol{r}^{q}-\boldsymbol{r}^{p}\right\|$ and $l_{0}^{(k)}$ is the reference length of the element. In addition two deformation coordinates $\varepsilon_{2}^{(k)}$ and $\varepsilon_{3}^{(k)}$, associated with the bending deformation of the beam element, can be defined [41]:

$$
\begin{aligned}
\varepsilon_{2}^{(k)}= & \mathcal{D}_{2}^{(k)}\left(\boldsymbol{x}_{\text {beam }}^{(k)}\right) \\
= & \frac{l_{0}^{(k)}}{l^{(k)}}\left(\left(x^{q}-x^{p}\right) \sin \left(\varphi^{p}+\beta_{0}\right)\right. \\
& \left.-\left(y^{q}-y^{p}\right) \cos \left(\varphi^{p}+\beta_{0}\right)\right), \\
\varepsilon_{3}^{(k)}= & \mathcal{D}_{3}^{(k)}\left(\boldsymbol{x}_{\text {beam }}^{(k)}\right) \\
= & -\frac{l_{0}^{(k)}}{l^{(k)}}\left(\left(x^{q}-x^{p}\right) \sin \left(\varphi^{q}+\beta_{0}\right)\right. \\
& \left.\quad-\left(y^{q}-y^{p}\right) \cos \left(\varphi^{q}+\beta_{0}\right)\right) .
\end{aligned}
$$

Note that in the expression for the elongation $\varepsilon_{1}^{(k)}$ second order geometric terms are included representing additional shortening of the beam axis caused by bending [67]. The deformation coordinates in Eq. (5.3) posses the proper invariance with respect to rigid body motions of the beam element.

The second example is the planar hinge element which can be used to describe the relative angle between two beam elements. The vector of nodal coordinates is

$$
\boldsymbol{x}_{\text {hinge }}^{(k)}=\left[\varphi^{p}, \varphi^{q}\right]^{\mathrm{T}},
$$

where $p$ and $q$ are the nodes at both sides of the hinge. There is a single deformation coordinate, which is the relative rotation angle $\varepsilon_{1}^{(k)}$ defined as

$$
\varepsilon_{1}^{(k)}=\mathcal{D}_{1}^{(k)}\left(\boldsymbol{x}_{\text {hinge }}^{(k)}\right)=\varphi^{q}-\varphi^{p} .
$$


For a description of deformation functions of spatial beam and hinge elements the reader is referred to $[12,38,41]$.

\subsubsection{Equations of motion}

A compliant mechanism or flexible manipulator can be built up with finite elements by letting them have nodal points in common. The assemblage of finite elements is realized by defining a vector $\boldsymbol{x}$ of nodal coordinates for the entire mechanism. The deformation functions of the elements can be described in terms of the components of the vector $\boldsymbol{x}$ yielding the non-linear vector function

$$
\varepsilon=\mathcal{D}(\boldsymbol{x}),
$$

which represents the basic equations for the kinematic analysis. Kinematic constraints can be introduced by putting conditions on the nodal coordinates $\boldsymbol{x}$ as well as by imposing conditions on the deformation coordinates $\varepsilon$ which are all assumed to be holonomic. For instance, rigidity of the elements can be enforced by imposing zero conditions on the subset of deformation coordinates associated with these elements, denoted by $\varepsilon^{(0)}=\mathcal{D}^{(0)}(\boldsymbol{x})=\mathbf{0}$.

An important notion in the kinematic and dynamic analysis of mechanisms is that of degrees of freedom. The number of kinematic degrees of freedom is the smallest number of coordinates that describe, together with the kinematic constraints, the configuration of the mechanism. We call them independent generalized coordinates which are denoted by the superscript $(m)$ and can be relative deformation coordinates, denoted by $\varepsilon^{(m)}$, or absolute nodal coordinates, denoted by $\boldsymbol{x}^{(m)}$. In this paper we choose to use only the deformation coordinates as independent generalized coordinates. The objective of kinematic analysis is then to solve Eq. (5.6) for the vector of independent generalized coordinates $\boldsymbol{q}=\boldsymbol{\varepsilon}^{(m)}$. The solution is expressed by means of geometric transfer functions $\mathcal{F}$ and $\mathcal{E}$ as

$$
\boldsymbol{x}=\mathcal{F}(\boldsymbol{q}), \quad \varepsilon=\mathcal{E}(\boldsymbol{q}) .
$$

The nodal velocities $\dot{\boldsymbol{x}}$ and accelerations $\ddot{\boldsymbol{x}}$ can be computed from (5.7) as

$$
\begin{gathered}
\dot{\boldsymbol{x}}=\frac{\partial \mathcal{F}}{\partial \boldsymbol{q}} \dot{\boldsymbol{q}}=\mathcal{F}_{, q} \dot{\boldsymbol{q}} \\
\ddot{\boldsymbol{x}}=\left(\frac{\partial^{2} \mathcal{F}}{\partial \boldsymbol{q}^{2}} \dot{\boldsymbol{q}}\right) \dot{\boldsymbol{q}}+\frac{\partial \mathcal{F}}{\partial \boldsymbol{q}} \ddot{\boldsymbol{q}}=\left(\mathcal{F}_{, \boldsymbol{q}} \dot{\boldsymbol{q}}\right) \dot{\boldsymbol{q}}+\mathcal{F}_{, q} \ddot{\boldsymbol{q}}
\end{gathered}
$$

Here the subscript notation ", $\boldsymbol{q}$ " is used to denote partial differentiation with respect to the vector of independent coordinates $\boldsymbol{q}{ }^{1}$ The geometric transfer functions $\mathcal{F}$ and $\mathcal{E}$ and their derivatives are determined numerically in an iterative way [38].

The inertia properties of the concentrated and distributed mass of the elements are described with the aid of configuration dependent lumped and consistent mass

\footnotetext{
${ }^{1}$ The notation $\left(\mathcal{F}_{\boldsymbol{q} \boldsymbol{q}} \dot{\boldsymbol{q}}\right) \dot{\boldsymbol{q}}$ and other similar expressions denote multiplication over the last dimension, i.e. $\mathcal{F}_{i, j k} \dot{q}_{j} \dot{q}_{k}$.
} 
matrices [38]. Let $\boldsymbol{M}_{\boldsymbol{x}}(\boldsymbol{x})$ be the global mass matrix, obtained by assembling the lumped and consistent element mass matrices, and let $\boldsymbol{f}(\boldsymbol{x}, \dot{\boldsymbol{x}}, t)$ be the vector of external nodal forces, including gravitational forces and the velocity dependent inertia forces. The loading state of each element is described by a vector of stress resultants. They are collected in the assembled vector $\boldsymbol{\sigma}$ which is dual to the vector of deformation coordinates $\varepsilon$. According to the principle of virtual work we obtain for the equations of motion of the mechanism

$$
\delta \boldsymbol{x}^{\mathrm{T}}\left(\boldsymbol{f}-\boldsymbol{M}_{\boldsymbol{x}} \ddot{\boldsymbol{x}}\right)=\delta \boldsymbol{\varepsilon}^{\mathrm{T}} \boldsymbol{\sigma},
$$

for all virtual variations $\delta \boldsymbol{x}$ and $\delta \varepsilon$ which satisfy the instantaneous kinematic constraints

$$
\delta \boldsymbol{x}=\mathcal{F}_{, \boldsymbol{q}} \delta \boldsymbol{q}, \quad \delta \varepsilon=\mathcal{E}_{, \boldsymbol{q}} \delta \boldsymbol{q} .
$$

Substituting Eqs. (5.8) and (5.10) in Eq. (5.9) gives

$$
\delta \boldsymbol{q}^{\mathrm{T}} \boldsymbol{M}_{\boldsymbol{q}} \ddot{\boldsymbol{q}}=\delta \boldsymbol{q}^{\mathrm{T}}\left(\mathcal{F}_{, \boldsymbol{q}}^{\mathrm{T}}\left(\boldsymbol{f}-\boldsymbol{M}_{\boldsymbol{x}}\left(\mathcal{F}_{, \boldsymbol{q} \boldsymbol{q}} \dot{\boldsymbol{q}}\right) \dot{\boldsymbol{q}}\right)-\mathcal{E}_{, \boldsymbol{q}}^{\mathrm{T}} \boldsymbol{\sigma}\right)
$$

with

$$
M_{\boldsymbol{q}}=\mathcal{F}_{, \boldsymbol{q}}^{\mathrm{T}} M_{\boldsymbol{x}} \mathcal{F}_{, \boldsymbol{q}}
$$

Since the components of the vector $\delta \boldsymbol{q}$ are independent, we obtain the equations of motion

$$
M_{\boldsymbol{q}} \ddot{\boldsymbol{q}}=\mathcal{F}_{, \boldsymbol{q}}^{\mathrm{T}}\left(\boldsymbol{f}-\boldsymbol{M}_{\boldsymbol{x}}\left(\mathcal{F}_{, \boldsymbol{q} q} \dot{\boldsymbol{q}}\right) \dot{\boldsymbol{q}}\right)-\mathcal{E}_{, \boldsymbol{q}}^{\mathrm{T}} \boldsymbol{\sigma}
$$

\subsubsection{Linearized equations of motion in a reference configuration}

Given the non-linear equations of motion (5.13), consider now small perturbations around some reference configuration specified by a set of degrees of freedom and its first and second time derivatives $\left(\boldsymbol{q}_{0}, \dot{\boldsymbol{q}}_{0}, \ddot{\boldsymbol{q}}_{0}\right)$ such that the actual variables are of the form

$$
\boldsymbol{q}=\boldsymbol{q}_{0}+\delta \boldsymbol{q}, \quad \dot{\boldsymbol{q}}=\dot{\boldsymbol{q}}_{0}+\delta \dot{\boldsymbol{q}}, \quad \ddot{\boldsymbol{q}}=\ddot{\boldsymbol{q}}_{0}+\delta \ddot{\boldsymbol{q}},
$$

where the prefix $\delta$ denotes a perturbation and should not be confused with the virtual variation operator in Eq. (5.9). Expanding the equations of motion (5.13) in their Taylor series expansion and disregarding second and higher order terms yields the linearized equations of motion [42],

$$
\boldsymbol{M}_{\boldsymbol{q}} \delta \ddot{\boldsymbol{q}}+\left[\boldsymbol{C}_{\boldsymbol{q}}+\boldsymbol{D}_{\boldsymbol{q}}\right] \delta \dot{\boldsymbol{q}}+\left[\boldsymbol{K}_{\boldsymbol{q}}+\boldsymbol{N}_{\boldsymbol{q}}+\boldsymbol{G}_{\boldsymbol{q}}\right] \delta \boldsymbol{q}=\mathcal{F}_{, \boldsymbol{q}}^{\mathrm{T}} \delta \boldsymbol{f}-\mathcal{E}_{, \boldsymbol{q}}^{\mathrm{T}} \delta \boldsymbol{\sigma}_{a}
$$

Here $\boldsymbol{C}_{\boldsymbol{q}}$ is the velocity sensitivity matrix, $\boldsymbol{D}_{\boldsymbol{q}}$ is the damping matrix, $\boldsymbol{K}_{\boldsymbol{q}}$ is the structural stiffness matrix and $\boldsymbol{N}_{\boldsymbol{q}}$ and $\boldsymbol{G}_{\boldsymbol{q}}$ are the dynamic stiffness and geometric stiffness matrices, respectively. Furthermore, $\delta \boldsymbol{f}$ and $\delta \boldsymbol{\sigma}_{a}$ represent time-varying perturbations of nodal forces and torques and internal driving forces and torques applied to the multibody system. For the special case when the system is at rest in 
an equilibrium configuration $\left(\dot{\boldsymbol{q}}_{0}=0, \ddot{\boldsymbol{q}}_{0}=0, \delta \boldsymbol{f}=0, \delta \boldsymbol{\sigma}_{a}=0\right)$, we can evaluate these matrices as follows ${ }^{2}$,

$$
\begin{aligned}
C_{\boldsymbol{q}}=\mathbf{0}, & \boldsymbol{N}_{\boldsymbol{q}}=\mathbf{0} \\
\boldsymbol{D}_{\boldsymbol{q}}=\mathcal{E}_{, \boldsymbol{q}}^{\mathrm{T}} \boldsymbol{D} \mathcal{E}_{, \boldsymbol{q}}, & \boldsymbol{K}_{\boldsymbol{q}}=\mathcal{E}_{, \boldsymbol{q}}^{\mathrm{T}} \boldsymbol{S \mathcal { E } _ { , q }}, \\
\boldsymbol{G}_{\boldsymbol{q}}=-\mathcal{F}_{, \boldsymbol{q}}^{\mathrm{T}} \boldsymbol{f}+\mathcal{E}_{, \boldsymbol{q}}^{\mathrm{T}} \boldsymbol{\sigma}, &
\end{aligned}
$$

where $\boldsymbol{S}, \boldsymbol{D}$ are the stiffness and damping matrices obtained by assembling the element stiffness and damping matrices. For expressions of the matrices in Eqs. (5.16) when the system is not at rest, the reader is referred to [42] or Appendix E.

\subsection{Reduction of degrees of freedom}

The equations of motion given by Eq. (5.13), need to be time-integrated to obtain the time response of the compliant mechanism or flexible manipulator. If high frequency modes are present in the model, explicit time-integrators require small time-steps to provide a stable solution, which reduces their computational efficiency. We will exemplify the cause of these high frequency modes and propose an approach to suppress them.

\subsubsection{Motivation and approach}

In Fig. 5.1, two examples are shown to illustrate the cause of the unwanted higher frequencies. In Fig. 5.1(a) a simply supported beam is considered where we would like to accurately model the illustrated high frequency bending mode. This requires a minimum of four elements as is shown. However, the model will now also include the generally much higher frequencies of unwanted elongation modes like the one shown in the figure. All elongation modes can be suppressed by prescribing all elongation deformations to be zero, but this is a too strong constraint if e.g. the first elongation mode should be included in the model and only the higher modes should be eliminated. In the second example of Fig. 5.1(b) a clamped beam is considered that undergoes large deflection. For an accurate representation that captures the geometric non-linear behaviour, one element is not enough as the small deformation assumption would be violated. Therefore, more elements are necessary to ensure small deformations in a co-rotational context, which again introduces high frequency modes.

In both cases, the computational efficiency can be significantly increased if these unwanted high frequency modes are suppressed. For this purpose, constraint relations between the degrees of freedom of the model can be applied. In the case of the simply supported beam, the elongation mode can be suppressed by e.g. relating the elongations of the separate beam elements. In general, the constraint relations can be non-linear, i.e. configuration dependent. In this paper we investigate configuration independent linear constraint relations of the form

$$
\boldsymbol{q}=\boldsymbol{V} \boldsymbol{z} \quad \text { with } \quad \operatorname{dim}(\boldsymbol{q})>\operatorname{dim}(\boldsymbol{z}),
$$

\footnotetext{
${ }^{2}$ The notation $\mathcal{F}_{, \boldsymbol{q}}^{\mathrm{T}} \boldsymbol{f}$ denotes multiplication over the first dimension of $\mathcal{F}_{\boldsymbol{q} \boldsymbol{q}}$, i.e. $\mathcal{F}_{i, j k} f_{i}$.
} 


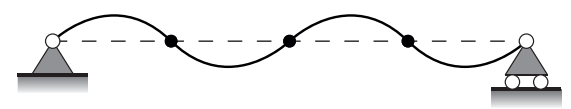

High frequency bending mode

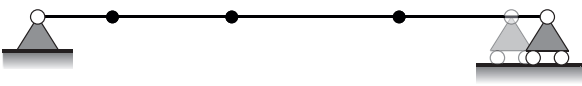

High frequency elongation mode

(a) Simply supported beam

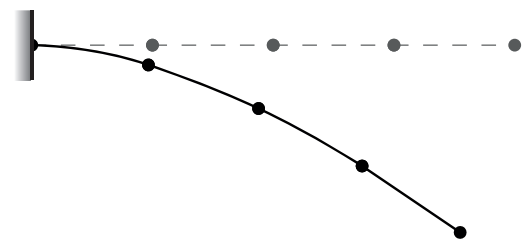

(b) Clamped beam

Figure 5.1: Examples of models that require multiple finite elements for accurate results: describing high frequency modes of a simply supported beam (a) and large deflections of a clamped beam (b).

where the columns of the projection matrix $\boldsymbol{V}$ span the allowable motion space with an associated reduced set of coordinates $\boldsymbol{z}$. Note that these linear constraints are only applied to the set of deformation coordinates contained in $\boldsymbol{q}$ and that the configuration and deformed state of the mechanism are subsequently determined with the non-linear geometric transfer functions of Eq. (5.7).

We can write Eq. (5.11) in terms of the reduced set of coordinates $\boldsymbol{z}$, by determining the virtual variations, and the first and second time derivative of Eq. (5.17) and substituting the resulting expressions, yielding

$$
\delta \boldsymbol{z}^{\mathrm{T}} \boldsymbol{M}_{\boldsymbol{z}} \ddot{\boldsymbol{z}}=\delta \boldsymbol{z}^{\mathrm{T}}\left[\mathcal{F}_{, \boldsymbol{q}} \boldsymbol{V}\right]^{\mathrm{T}}\left(\boldsymbol{f}-\boldsymbol{M}_{\boldsymbol{x}}\left(\mathcal{F}_{, \boldsymbol{q} q} \boldsymbol{V} \dot{\boldsymbol{z}}\right) \boldsymbol{V} \dot{\boldsymbol{z}}\right)-\delta \boldsymbol{z}^{\mathrm{T}}\left[\mathcal{E}_{, \boldsymbol{q}} \boldsymbol{V}\right]^{\mathrm{T}} \boldsymbol{\sigma},
$$

with

$$
\boldsymbol{M}_{\boldsymbol{z}}=\left[\mathcal{F}_{, \boldsymbol{q}} \boldsymbol{V}\right]^{\mathrm{T}} \boldsymbol{M}_{\boldsymbol{x}}\left[\mathcal{F}_{, \boldsymbol{q}} \boldsymbol{V}\right]
$$

The components of $\delta \boldsymbol{z}$ are independent and therefore we can write for the reduced equations of motion

$$
\boldsymbol{M}_{\boldsymbol{z}} \ddot{\boldsymbol{z}}=\left[\mathcal{F}_{, \boldsymbol{q}} \boldsymbol{V}\right]^{\mathrm{T}}\left(\boldsymbol{f}-\boldsymbol{M}_{\boldsymbol{x}}\left(\mathcal{F}_{, \boldsymbol{q} \boldsymbol{}} \boldsymbol{V} \dot{\boldsymbol{z}}\right) \boldsymbol{V} \dot{\boldsymbol{z}}\right)-\left[\mathcal{E}_{, \boldsymbol{q}} \boldsymbol{V}\right]^{\mathrm{T}} \boldsymbol{\sigma} .
$$

This leaves the determination of the projection matrix $\boldsymbol{V}$. In this paper we propose two approaches: a projection method using a modal basis, and a method where we determine the modeshapes in a variety of configurations after which the dominant contributions are obtained from an analysis using singular value decomposition (SVD).

\subsubsection{Modal basis}

For determining a modal basis to suppress the unwanted higher frequency modes, we first partition Eq. (5.17) as follows

$$
\left\{\begin{array}{c}
\boldsymbol{q}_{l} \\
\boldsymbol{q}_{s}
\end{array}\right\}=\left[\begin{array}{ll}
\boldsymbol{V}_{l} & \\
& \boldsymbol{V}_{s}
\end{array}\right]\left\{\begin{array}{l}
\boldsymbol{z}_{l} \\
\boldsymbol{z}_{s}
\end{array}\right\}
$$


where the subscript $l$ denotes coordinates that can undergo large rotations such as the relative angle of a hinge, and the subscript $s$ denotes coordinates that remain small during simulation such as the deformation coordinates of the beam element. The reason for this partitioning is that usually these deformation coordinates show quite distinct behaviour in relation to a modal analysis. The large rotations in $\boldsymbol{q}_{l}$ typically result in highly non-linear behaviour that cannot be adequately described by a reduced set of coordinates using a linear projection. Therefore, it is assumed that there is no coupling between the two type of coordinates and modal reduction is only applied to the $\boldsymbol{q}_{s}$ part. More specifically, the submatrix $\boldsymbol{V}_{l}$ is chosen to be the identity matrix such that $\boldsymbol{q}_{l}=\boldsymbol{z}_{l}$. The submatrix $\boldsymbol{V}_{s}$ is determined by prescribing $\boldsymbol{q}_{l}=\mathbf{0}$ after which the eigenfrequencies and eigenvectors are obtained by solving the eigenvalue problem

$$
\left(\left[\boldsymbol{K}_{\boldsymbol{q} s s}+\boldsymbol{G}_{\boldsymbol{q} s s}\right]-\omega_{i}^{2} \boldsymbol{M}_{\boldsymbol{q} s s}\right) \boldsymbol{v}_{i}=\mathbf{0},
$$

in the initial configuration for a subset of the linearized equations of motion (5.15). Here the subscript $s$ indicates the part of the system matrices associated with $\boldsymbol{q}_{s}$ and $\omega_{i}$ is the $i^{\text {th }}$ eigenfrequency with corresponding eigenvector $\boldsymbol{v}_{i}$ that is normalized and collected in the $i^{t h}$ column of the submatrix $\boldsymbol{V}_{s}$. Model order reduction is achieved by omitting the eigenvectors corresponding to unwanted high frequency vibrational modes in $\boldsymbol{V}_{\boldsymbol{s}}$.

This last step is however not trivial for non-linear systems, where in general the vibrational modes are configuration dependent. This is especially evident in compliant mechanisms that undergo large deflections. To cope with this non-linear behaviour, some of the higher frequency vibrational modes can be kept in $\boldsymbol{V}_{s}$. Though, choosing which modes to keep is not a trivial task.

\subsubsection{SVD-modal basis}

A more systematic approach to determine a projection base that is adequate in the operating range of the mechanism is outlined by the following procedure:

1. In the working range of the mechanism, we determine the linearized equations of motion (5.15) in $n$ equidistant reference configurations and obtain for each reference configuration a normalized modal basis, $\boldsymbol{V}_{s}^{(i)}$. The superscript $i$ refers to the $i^{\text {th }}$ reference configuration with $i=1,2, \ldots, n$.

2. In reference configuration $i$, we retain the first $r$ modes in $\boldsymbol{V}_{s}^{(i)}$ that should be accurately described.

3. The modes are weighted by their eigenfrequencies as follows,

$$
\boldsymbol{U}_{s}^{(i)}=\boldsymbol{V}_{s}^{(i)} \boldsymbol{W}^{(i)},
$$

with

$$
\boldsymbol{W}^{(i)}=\left[\begin{array}{lll}
\omega_{1}^{-a} & & \\
& \ddots & \\
& & \omega_{r}^{-a}
\end{array}\right],
$$


where $\boldsymbol{U}_{s}^{(i)}$ is the weighted set of $r$ modes for the $i^{\text {th }}$ configuration and $a$ is the weight parameter that scales the weighting. E.g. for $a=0$ there is no weighting and for $a=1$ the weight on the modes reduces linearly with the eigenfrequency. In fact, this parameter can take any real value and is discussed in somewhat more detail in the next section.

4. Concatenate the matrices $\boldsymbol{U}_{s}^{(i)}$ with $i=1,2, \ldots, n$ and form

$$
\boldsymbol{Q}_{s}=\left[\boldsymbol{U}_{s}^{(1)}, \boldsymbol{U}_{s}^{(2)}, \ldots, \boldsymbol{U}_{s}^{(n)}\right]
$$

5. Perform an SVD of the matrix $\boldsymbol{Q}_{s}$ and retain the left singular vectors ${ }^{3}$ with large corresponding singular values to form the projection matrix $\boldsymbol{V}_{s}$ for the reduction of coordinates as in Eq. (5.20).

In the first step, the equilibrium reference configurations are chosen to be obtained equidistantly along the direction of motion. This is to ensure that no configuration bias is introduced in the SVD analysis of step 5 . Also, to avoid bias, only normalized eigenvectors, or alternatively eigenvectors with the same length, are used in this step.

The weighting by the parameter $a$ in the third step can be used to give higher priority to the lower frequency vibrational modes in the SVD analysis.

\subsection{Examples}

Two planar examples are used to demonstrate the proposed approach. A compliant straight guidance example is included to show its effectiveness on the model reduction for compliant mechanisms that undergo large deflections. And as a second example, a flexible manipulator is considered to demonstrate the applicability of the reduction procedure to a more general class of mechanisms. In both examples the equations of motion are numerically integrated with MATLAB's ode45 integrator, which is an explicit fourth order variable step-size integrator [25].

\subsubsection{Compliant straight guidance}

Consider the compliant straight guidance in Fig. 5.2, which consists of two flexures each modelled with five flexible planar beam elements connected by a rigid intermediate body. Each beam element in the flexures allows for two bending deformations, resulting in a model with 17 degrees of freedom that all remain small in a co-rotational context. The physical properties and dimensions are given in Tab. 5.1. The model is excited by a force as function of time

$$
f(t)=\left\{\begin{array}{lr}
\frac{F_{\max }}{2}(1-\cos (20 \pi t)), & 0 \mathrm{~s} \leq t \leq 0.1 \mathrm{~s}, \\
0, & t>0.1 \mathrm{~s},
\end{array}\right.
$$

\footnotetext{
${ }^{3}$ The SVD of a matrix $\boldsymbol{A}$ is given by: $\boldsymbol{A}=\boldsymbol{U} \boldsymbol{\Sigma} \boldsymbol{V}^{\mathrm{T}}$, where $\boldsymbol{\Sigma}$ is a diagonal matrix of singular values and $\boldsymbol{U}$ and $\boldsymbol{V}$ are the matrices with the left and right singular vectors, respectively.
} 


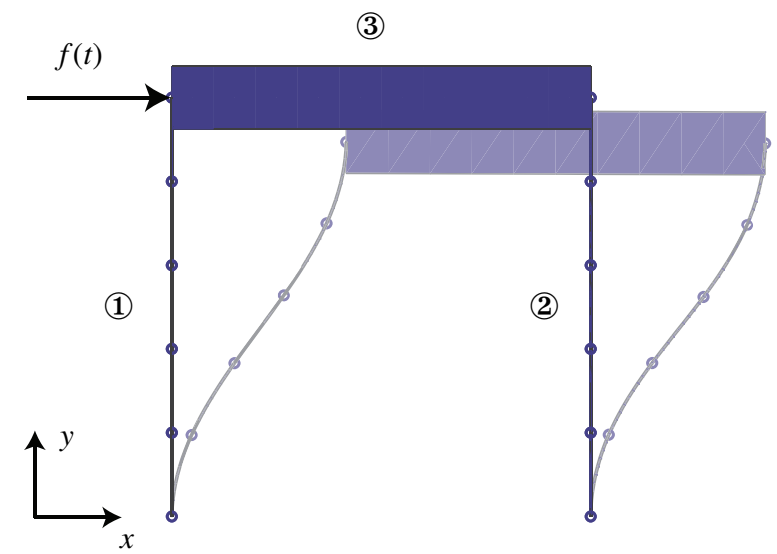

Figure 5.2: Two-dimensional straight guidance model. Both flexures are clamped at the bottom end.

Table 5.1: Physical properties and dimensions of the two-dimensional straight guidance model.

\begin{tabular}{lll}
\hline Property & Flexures (1) (2) & Rigid beam (3) \\
\hline Length, $l$ & $0.2 \mathrm{~m}$ & $0.2 \mathrm{~m}$ \\
Cross-sectional area, $A$ & $30 \cdot 10^{-6} \mathrm{~m}^{2}$ & $9 \cdot 10^{-4} \mathrm{~m}^{2}$ \\
Cross-sectional area & & \\
$\quad$ moment of inertia, $I$ & $2.5 \cdot 10^{-12} \mathrm{~m}^{4}$ & $6.75 \cdot 10^{-8} \mathrm{~m}^{4}$ \\
Young's Modulus, $E$ & $2.1 \cdot 10^{11} \mathrm{~N} / \mathrm{m}^{2}$ & \\
Density, $\rho$ & $7600 \mathrm{~kg} / \mathrm{m}^{3}$ & $7600 \mathrm{~kg} / \mathrm{m}^{3}$ \\
\hline
\end{tabular}

where the maximal applied force is $F_{\max }=100 \mathrm{~N}$.

We simulate the time response of the unreduced non-linear model and compute the eigenfrequencies as a function of the $x$-displacement of the rigid intermediate body. This simulation will serve as a benchmark for the reduced models. In Fig. 5.3(a), the time response of the $x$-displacement of the rigid intermediate body is shown. Here the region between the dashed lines indicates the full range of motion of the compliant mechanism.

For the reduced models, we would like to retain the time response of the compliant straight guidance and the second and third eigenfrequencies over the full range of motion. Four reduced models are considered. First, by using the modal basis as discussed in Section 5.3.2, a reduced model is created with three time invariant modal modes determined in the initial configuration and is labelled as "MODAL-3". We also consider a reduced model with five time invariant modal modes, labelled as "MODAL-5". The other two reduced models are obtained by using the SVD-modal basis approach of Section 5.3.3. Here we applied an SVD analysis to a set of modes 


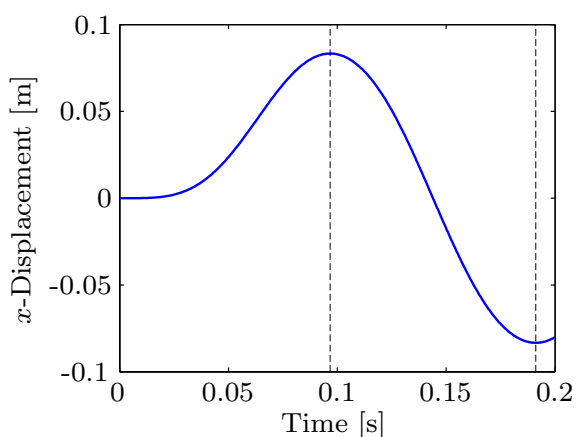

(a) $x$-Displacement as function of time

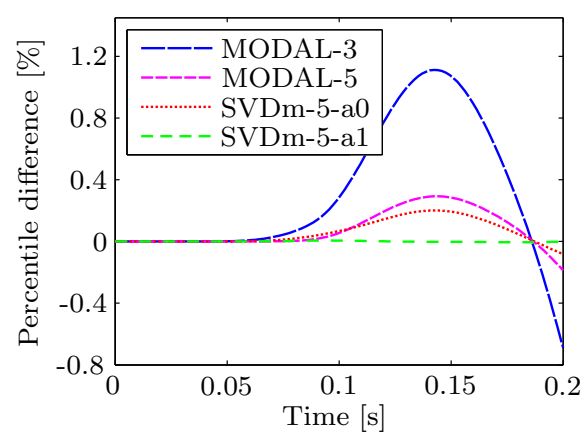

(b) Relative $x$-displacement differences with respect to maximal deflection $(0.0833 \mathrm{~m})$

Figure 5.3: Time response of the $x$-displacement of the rigid intermediate body of the unreduced model (a) and the relative time response errors of the reduced models computed with respect to the maximal deflection of $0.0833 \mathrm{~m} \mathrm{(b)}$.

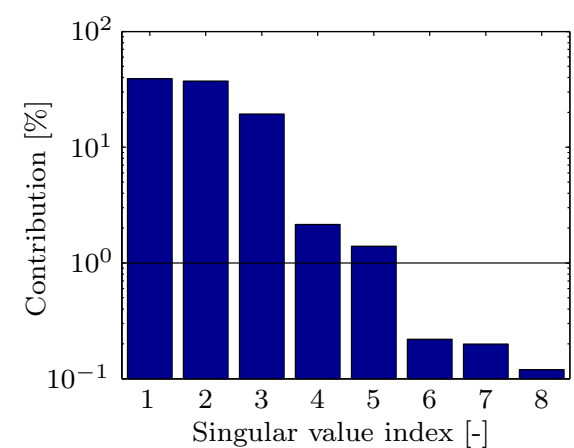

(a) No weighting $a=0$

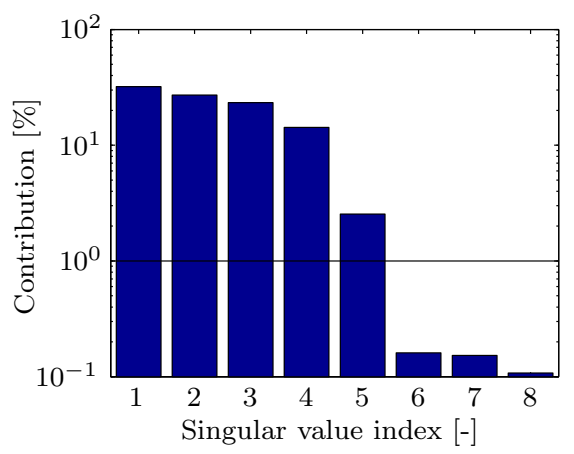

(b) With weighting $a=1$

Figure 5.4: Singular values scaled with the sum of all singular values, for an SVD analysis using ten configurations of the straight guidance with no weighting (a) and with weighting (b).

consisting of the first three modes in ten equidistant reference configurations over the full range of motion. The distinction between the two SVD-modal models is in the weighting parameter of the vibrational modes for the SVD analysis, where we consider a case with no weighting $(a=0)$ and a case of linear weighting with the eigenfrequencies $(a=1)$. The resulting singular values of both SVD analyses are shown in Fig. 5.4. The singular values are scaled with the sum of all singular values 


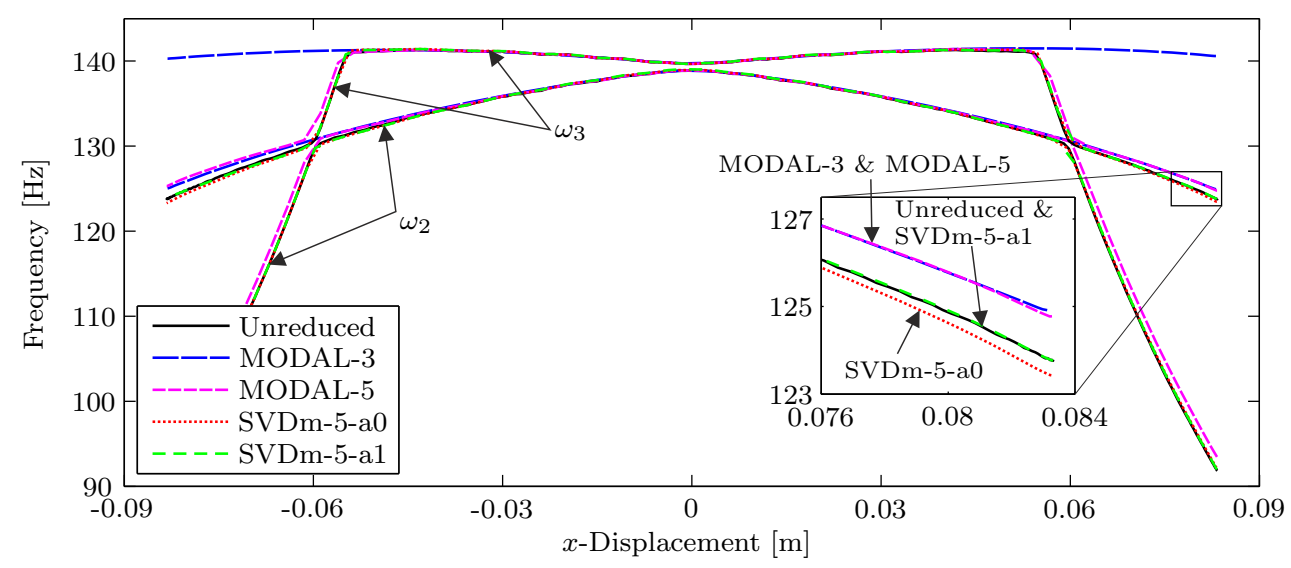

Figure 5.5: Second $\left(\omega_{2}\right)$ and third $\left(\omega_{3}\right)$ eigenfrequencies of the straight guidance as a function of the full range of motion: comparison of the unreduced and reduced models.

such that the relative contribution of each singular vector is immediately clear. We retain singular vectors with a contribution greater than $1 \%$. For both weighting cases it appears that five modes are necessary to capture the second and third vibrational modes over the full range of motion. These models are labelled "SVDm-5-a0" and "SVDm-5-a1", respectively, to indicate the number of modes and the weighting. It should be noted that the $1 \%$ criterion is used for this example, yet it is not meant to be a general rule.

Fig. 5.3(b) shows the relative differences between the unreduced and the reduced models of the time response in $x$-direction of the rigid intermediate body. All four reduced models show good agreement with the unreduced model. The SVD-modal models show better performance than the modal models. For the SVD-modal models, it is clear that by giving higher priority to the lower frequency vibrational modes by setting the weighting parameter $a=1$, the time response error is reduced most. Note that increasing the parameter $a$ too much can give undesirable results for the second and third vibrational modes as all the emphasis in the SVD analysis will be on the first vibrational mode.

In Fig. 5.5 the second and third eigenfrequencies of the unreduced and reduced models are shown. They are computed over the full range of motion as is indicated by the region between the dashed lines of Fig. 5.3(a). The MODAL-3 model is able to correctly capture the second and third eigenfrequencies up to a deflection of approximately $0.055 \mathrm{~m}$. After that, due to mode veering of previously higher frequency modes, it is not able to correctly describe the second vibrational mode anymore. This is the reason why the SVD analysis for the SVD-modal models suggested five modes to be used as a reduction basis. From the results of the MODAL-5, SVDm5-a0 and SVDm5-a1 reduced models, it can be concluded that 
this indeed gives accurate results over the full range of motion. We note that the SVD-modal models are outperforming the MODAL-5 model as they take into account the configuration dependency of the eigenvectors over the full range of motion. Also, we note that a slightly more accurate model is obtained when the eigenvectors are weighted by their eigenfrequencies in the SVD analysis. These results show that the geometric non-linear effects are correctly preserved in the reduced models.

The number of function evaluations required by the explicit variable step-size integrator for the MODAL-3, MODAL-5, SVDm-5-a0 and SVDm-5-a1 are respectively $697,1213,1411$ and 1303. In contrast, the unreduced model required 46075 function evaluations. This illustrates a high increase in computational efficiency for the reduced models.

\subsubsection{Flexible manipulator}

Consider the two-link flexible manipulator shown in Fig. 5.6. This manipulator has been introduced as a benchmark by Schiehlen and Leister [57] and has been quoted in several papers. Some properties are given in Tab. 5.2. Different from the original benchmark, we do not include gravity in this paper. Also, instead of prescribing the joint angles with rheonomic constraints, we apply a stiff proportional controller with a gain $k_{p}=5 \cdot 10^{3} \mathrm{Nm} / \mathrm{rad}$, in each of the joints. The reference for the controllers are the joint angles $\phi_{1}(t)$ and $\phi_{2}(t)$ that are defined by third order functions of time

$$
\phi_{1}(t)=-\phi_{2}(t)=\left\{\begin{array}{lr}
\pi / 4\left(-1+72 t^{3}\right), & 0 \mathrm{~s} \leq t<1 / 6 \mathrm{~s}, \\
\pi / 4\left(-18 t+108 t^{2}-144 t^{3}\right), & 1 / 6 \mathrm{~s} \leq t<1 / 3 \mathrm{~s}, \\
\pi / 4\left(-8+54 t-108 t^{2}+72 t^{3}\right), & 1 / 3 \mathrm{~s} \leq t<1 / 2 \mathrm{~s}, \\
\pi / 4, & 1 / 2 \mathrm{~s} \leq t .
\end{array}\right.
$$

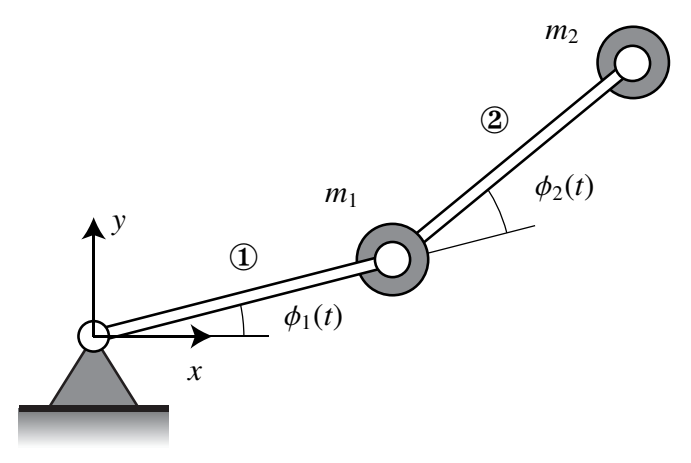

Figure 5.6: Planar two-link manipulator model. 
Table 5.2: Physical properties and dimensions of the planar two-link manipulator model (adapted from [57]).

\begin{tabular}{lll}
\hline Property & Link (1) & Link (2) \\
\hline Length, $l$ & $0.545 \mathrm{~m}$ & $0.675 \mathrm{~m}$ \\
Joint mass, $m$ & $1.0 \mathrm{~kg}$ & $3.0 \mathrm{~kg}$ \\
Cross-sectional area, $A$ & $9.0 \cdot 10^{-4} \mathrm{~m}^{2}$ & $4.0 \cdot 10^{-4} \mathrm{~m}^{2}$ \\
Cross-sectional area & & \\
$\quad$ moment of inertia, $I$ & $1.69 \cdot 10^{-8} \mathrm{~m}^{4}$ & $3.33 \cdot 10^{-9} \mathrm{~m}^{4}$ \\
Young's Modulus, $E$ & $7.3 \cdot 10^{10} \mathrm{~N} / \mathrm{m}^{2}$ & $7.3 \cdot 10^{10} \mathrm{~N} / \mathrm{m}^{2}$ \\
Density, $\rho$ & $2700 \mathrm{~kg} / \mathrm{m}^{3}$ & $2700 \mathrm{~kg} / \mathrm{m}^{3}$ \\
\hline
\end{tabular}

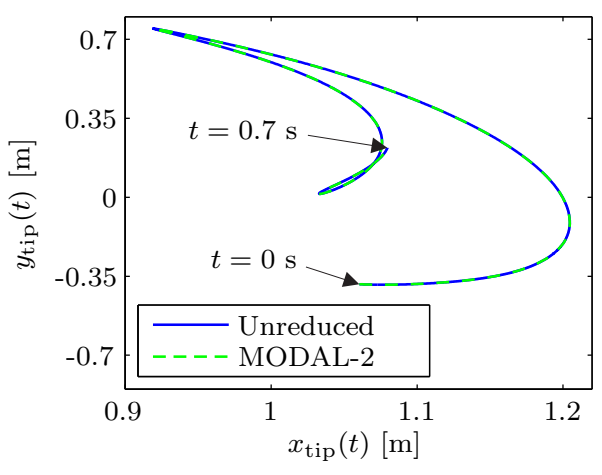

(a) Tip response

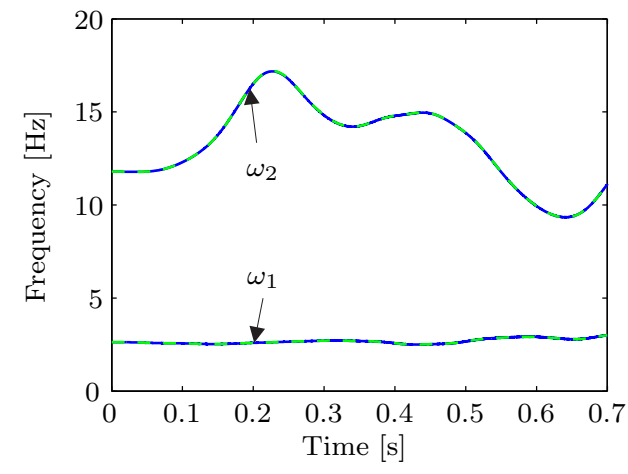

(b) Eigenfrequencies as a function of time

Figure 5.7: Motion of the manipulator tip during $0.7 \mathrm{~s}$ of simulation (a) and the first $\left(\omega_{1}\right)$ and second $\left(\omega_{2}\right)$ eigenfrequencies as functions of time (b) for the reduced and unreduced models.

The motion of this manipulator has been computed with a non-linear model in which four flexible planar beam elements are used for each link. Each beam allows two bending modes yielding a model with eighteen degrees of freedom including the two large rotational degrees of freedom of the hinges. Next a reduced model "MODAL-2" is created using two modal modes determined in the initial configuration. Here the deformation coordinates in the beam elements are only reduced as they belong to the category of coordinates $\boldsymbol{q}_{s}$ from Eq. (5.20). The degrees of freedom $\boldsymbol{q}_{l}$ are both joint rotations that are not included in the modal analysis as outlined in Section 5.3.2. From the results in Fig. 5.7 it is clear that the reduced model with only four degrees of freedom is able to accurately predict the tip response and the first and second eigenfrequencies. The computational time is significantly reduced, as the reduced model required only 5113 function evaluations for the simulation whereas the unreduced model required 345145 
function evaluations.

Note that we have not considered the SVD-modal approach in this example. It is thought that in this case only a marginal increase in performance can be obtained as the deflection of the links is already correctly captured using the first two modal modes from the initial configuration. Furthermore, most of the non-linearity arises from varying the hinge coordinates which are not reduced.

\subsection{Conclusions}

In this paper a reduction method is proposed for the efficient explicit time-integration of flexible multibody models. For suppressing high frequency modes, linear constraint relations are applied to the independent generalized coordinates that remain small during simulation. Geometric non-linear effects are retained by the geometric transfer functions that describe the configuration of the mechanism in terms of the independent coordinates.

The approach is demonstrated by two planar examples: a compliant straight guidance that undergoes large deflection and a flexible manipulator. In both examples it is observed that significant changes in eigenfrequencies during simulation are correctly described by the reduced models. In the case of the compliant straight guidance, mode veering of high frequency modes occurs for rather large deflections. To maintain accuracy for these large deflections, an SVD-modal basis is applied that is derived from an SVD analysis of normalized eigenmodes in a variety of configurations. It is shown that by weighting the eigenmodes with their eigenfrequencies in the SVD analysis, more accurate results are obtained than when no weighting is applied. A significant increase in computational efficiency is observed for the time-integration of the reduced equations of motion.

It should be noted that the reduction methods outlined in this paper appeared to be adequate for the presented planar flexible multibody systems. For other classes of systems like spatial compliant systems somewhat different approaches may be more successful [12] (see Chapter 6).

Acknowledgements This research is financially supported by the Dutch association Point-One, project MOV-ET PNE08006, of the Dutch Department of Economic Affairs, Agriculture and Innovation. 


\title{
Model reduction for efficient time-integration of spatial flexible multibody models
}

\begin{abstract}
A reduction method is proposed for efficient time-integration of compliant mechanism models that undergo large deflections. Of particular importance for the modelling of this class of mechanisms is the accurate description of geometric nonlinearities, as stiffness characteristics can change significantly during deflection. A finite element based flexible multibody approach is used to describe the compliant mechanism in terms of independent generalized coordinates. The modelling of large deflections requires a sufficient number of finite elements to ensure that deformations remain small in a co-rotational context. Increasing the number of elements, increases, besides the number of degrees of freedom, the largest eigenfrequency in the model. This reduces the allowable step size for explicit time-integrator methods. The proposed reduction method aims to suppress the high frequency vibrational modes which are not important for the desired simulation results, while retaining the geometric non-linearities in the reduced model. For this purpose we add constraint relations between the independent generalized coordinates. These constraint relations can be linear or non-linear. Both cases are investigated in this paper and are implemented as a fixed and an interpolated basis method, respectively. The effectiveness of the two methods is demonstrated by a simulation of a compliant straight guidance in a gravity field that undergoes large deflection. Both methods can yield accurate results with a significant increase in computational efficiency.
\end{abstract}

\subsection{Introduction}

Compliant mechanisms with flexure based hinges are often utilized in high precision manipulator devices for their deterministic static and dynamic behaviour. High accuracies can be attained due to the absence of friction and low hysteresis. In

Published in: Multibody System Dynamics (S.E. Boer, R.G.K.M. Aarts, W.B.J. Hakvoort) 
this paper, we consider compliant mechanisms that undergo large deflections. The analysis for design and control of such devices relies on computationally efficient models that are capable of capturing the relevant dynamic and compliant characteristics over the full range of motion. This requires the sound inclusion of geometric non-linear effects.

In [2] we discuss a modelling approach for the analysis and simulation of multibody systems with flexible components where an entire multibody system is modelled as the assembly of non-linear finite elements. For each element a fixed number of deformation modes are defined with associated deformation coordinates that are invariant for arbitrary rigid body motion. Typical flexible members of compliant mechanisms are wire and sheet flexures. An example of such a mechanism, is the two degrees of freedom large-stroke compliant mechanism presented by Folkersma et al. [27]. This mechanism contains eleven cross flexure hinges, each containing five sheet flexures, to realize two translational in-plane degrees of freedom. These sheet flexures may be considered as one-dimensional structures which can be correctly modelled by flexible beam elements. One element could be sufficient if only small deformations are considered. However, for large deformations, an adequate number of flexible beam elements are required to correctly capture the geometric non-linear effects. For the large-stroke compliant mechanism of [27], typically four flexible beam elements are required for the modelling of each sheet flexure in the eleven cross flexure hinges. Consequently, high frequency modes are introduced in the model that greatly deteriorate the computational efficiency of explicit integrators for the time-integration of the non-linear equations of motion. Whereas, only the first few vibrational modes up to a certain frequency are expected to be of interest for e.g. closed-loop control simulations.

For linear mechanical systems, these higher frequency modes can be removed by using model reduction techniques that project the dynamics onto a lower dimensional subspace while preserving the dominant dynamical behaviour. Research on model reduction techniques applied to non-linear flexible multibody systems can be separated into two categories: component and system level model reduction. Component level reduction techniques are employed to obtain reusable, efficient models of complex-shaped components [11, 21, 48, 59], whereas system level model reduction is used to improve the computational efficiency on a global level. The adaptive modal integration (AMI) method introduced by Aarts and Jonker [1] and the global modal parameterization (GMP) method introduced by Brüls et al. [18] are examples of system level model reduction techniques. The GMP method is further developed by Naets et al. $[54,55]$ for real-time applications. Both the GMP and the AMI methods separate the motion of the mechanism in a non-linear nominal rigid body motion and a configuration dependent elastic motion part. The reduction is realized by reducing the number of the equations necessary to describe the elastic motion. One of the main differences between the two methods lies in the equations of motion they are applied to. The AMI method is applied to a set of non-linear equations of motion expressed as ordinary differential equations (ODE) for a set of independent coordinates, as in [2]. Whereas the GMP method is applied 
to a general set of differential algebraic equations (DAE), from which a cheaper to solve set of ODE's is obtained by combining the linearized models with the constraint equations. Both methods in their current form assume that the elastic deformations remain small with respect to the rigid body motion. Consequently, they are less suited to reduce models describing compliant mechanisms that undergo large deflections in which geometric non-linear stiffening effects play a dominant role.

Therefore, we aim to reduce the non-linear equations of motion of a multibody system with flexible components, such that the geometric non-linear effects and the vibrational modes up to a certain frequency, are retained. For this purpose we consider the modelling approach discussed in [2], where geometric transfer functions are used to express the equations of motion as ODE's for a set of independent generalized coordinates. The geometric transfer functions describe the configuration and deformation state of the mechanism as explicit functions of the independent generalized coordinates. In the proposed reduction method, the number of independent coordinates is reduced by imposing constraint relations between them. This is different from the AMI and GMP methods, where reduced linearized models are interpolated. We consider two types of constraint relation, linear and non-linear, resulting in a fixed basis method and an interpolated basis method, respectively.

In the fixed basis method, the reduction of coordinates is realized by projecting the independent generalized coordinates onto a lower dimensional subspace.

For the interpolated basis method, non-linear constraint relations are formed by writing the independent generalized coordinates as a superposition of the gross reference motion and the vibrational modes of interest. The subspace spanned by the vibrational modes of interest is interpolated over the trajectory of motion, such that the configuration dependency of these modes is correctly described. In [33] it is demonstrated that direct interpolation of the eigenvectors can yield undesirable results due to mode-crossing and mode-veering phenomena [56]. Different interpolation strategies, based on mode-tracking algorithms, have been proposed to cope with mode-crossing and mode-veering [5, 18]. As an alternative, we propose a so-called modal subspace interpolation (MSI) algorithm. Here, the base vectors of the modal subspaces are chosen such that they span the configuration dependent subspace while they vary as little as possible with a configuration change. This allows easy interpolation of the modal subspaces. In this paper we consider one coordinate that describes the configuration change. Though, an extension to multiple configuration coordinates is thought to be possible.

The contents of this paper are organized as follows. In Section 6.2 the finite element representation used in this paper for the modelling of multibody models of large-stroke compliant mechanisms is briefly explained. Then in Section 6.3, the reduced equations of motion are derived and the fixed and interpolated basis method are introduced. The effectiveness of the two methods is demonstrated in Section 6.4 by a simulation of a compliant straight guidance in a gravity field that undergoes large deflections. 


\subsection{Finite element modelling}

In this section we summarize the most important aspects for this paper of the modelling approach previously published in [38, 41]. Here, we will elaborate on the specification of independent deformation modes of a non-linear flexible beam element. Also, the concept of geometric transfer functions is explained, which is used to derive the equations of motion in terms of independent generalized coordinates.

\subsubsection{Finite element representation}

In the presented finite element method, a compliant mechanism is modelled as an assembly of non-linear spatial finite beam elements [38]. The location of each beam element is described relative to a fixed inertial coordinate system, $\left[\boldsymbol{n}_{x}, \boldsymbol{n}_{y}, \boldsymbol{n}_{z}\right]$, by a set of nodal coordinates $\boldsymbol{x}^{(k)}$, valid for large displacements and rotations. Here the superscript $k$ is added to show that a specific element $k$ is considered. As nodal coordinates we have two sets of Cartesian coordinates, $\boldsymbol{r}^{p}$ and $\boldsymbol{r}^{q}$, describing the nodal positions in the inertial coordinate system and two sets of Euler parameters, $\boldsymbol{\lambda}^{p}$ and $\boldsymbol{\lambda}^{q}$, representing the orientation of the triads $\left[\boldsymbol{e}_{x}^{p}, \boldsymbol{e}_{y}^{p}, \boldsymbol{e}_{z}^{p}\right]$ and $\left[\boldsymbol{e}_{x}^{q}, \boldsymbol{e}_{y}^{q}, \boldsymbol{e}_{z}^{q}\right]$ rigidly attached at nodes $p$ and $q$, see Fig. 6.1. The vector $\boldsymbol{x}^{(k)}$ of nodal coordinates can then be written as

$$
\boldsymbol{x}^{(k)}=\left\{\boldsymbol{r}^{p \mathrm{~T}}, \quad \boldsymbol{\lambda}^{p \mathrm{~T}}, \quad \boldsymbol{r}^{q \mathrm{~T}}, \quad \boldsymbol{\lambda}^{q \mathrm{~T}}\right\}^{\mathrm{T}} .
$$

Note that the parametrization of rotations is redundant due to the constraint $\boldsymbol{\lambda}^{\mathrm{T}} \boldsymbol{\lambda}=$ 1, imposed on the Euler parameters of Eq. (6.1). This results in a total of twelve

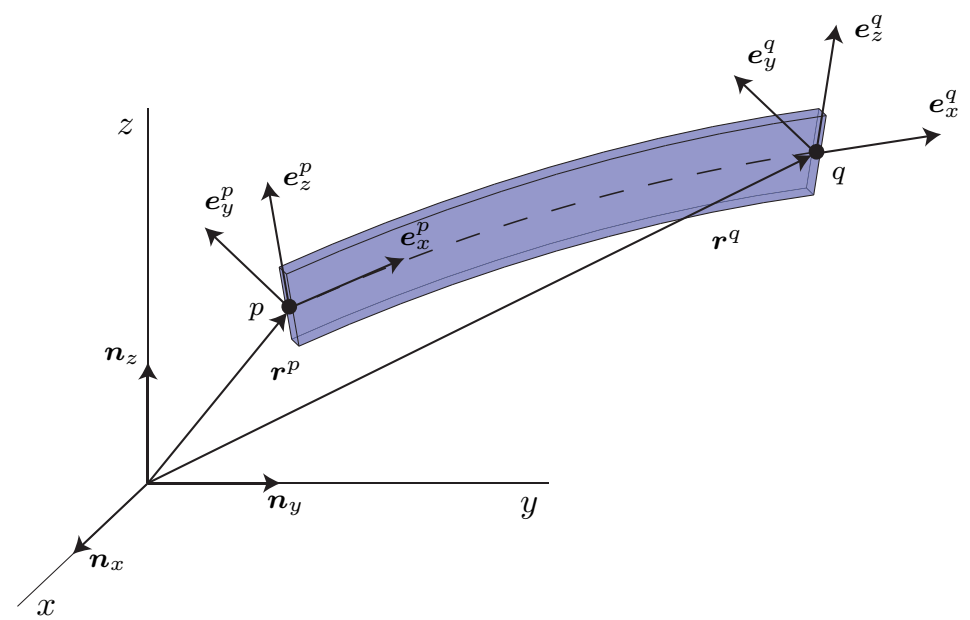

Figure 6.1: The configuration of the non-linear spatial beam element expressed in the inertial reference frame $\left[\boldsymbol{n}_{x}, \boldsymbol{n}_{y}, \boldsymbol{n}_{z}\right]$. 
independent nodal coordinates.

With respect to some reference configuration of the element, the instantaneous values of the nodal coordinates represent a fixed number of deformation modes for the element. As the beam element has six degrees of freedom as a rigid body and twelve independent nodal coordinates, six independent deformation modes, specified by a set of deformation coordinates $\varepsilon^{(k)}$, can be expressed as analytical functions of the vector of nodal coordinates $\boldsymbol{x}^{(k)}$ and the reference length $l_{0}^{(k)}[41]$,

$$
\varepsilon^{(k)}=\mathcal{D}^{(k)}\left(\boldsymbol{x}^{(k)}\right),
$$

where

$$
\begin{aligned}
& \varepsilon_{1}^{(k)}=l-l_{0}^{(k)} \\
& \varepsilon_{2}^{(k)}=l_{0}^{(k)}\left(\boldsymbol{e}_{z}^{p} \cdot \boldsymbol{e}_{y}^{q}-\boldsymbol{e}_{y}^{p} \cdot \boldsymbol{e}_{z}^{q}\right) / 2, \\
& \varepsilon_{3}^{(k)}=-l_{0}^{(k)} \boldsymbol{e}_{l} \cdot \boldsymbol{e}_{z}^{p} \\
& \varepsilon_{4}^{(k)}=l_{0}^{(k)} \boldsymbol{e}_{l} \cdot \boldsymbol{e}_{z}^{q} \\
& \varepsilon_{5}^{(k)}=l_{0}^{(k)} \boldsymbol{e}_{l} \cdot \boldsymbol{e}_{y}^{p} \\
& \varepsilon_{6}^{(k)}=-l_{0}^{(k)} \boldsymbol{e}_{l} \cdot \boldsymbol{e}_{y}^{q}
\end{aligned}
$$

with

$$
l=\left\|\boldsymbol{r}^{q}-\boldsymbol{r}^{p}\right\| \quad \text { and } \quad \boldsymbol{e}_{l}=\left(\boldsymbol{r}^{q}-\boldsymbol{r}^{p}\right) / l^{(k)} .
$$

Here $\boldsymbol{e}_{y}^{p}, \boldsymbol{e}_{z}^{p}, \boldsymbol{e}_{y}^{q}$ and $\boldsymbol{e}_{z}^{q}$ correspond to the base vectors of the orthonormal triads attached to the nodes of the element. The first deformation coordinate, $\varepsilon_{1}$, describes the elongation of the element, the second one, $\varepsilon_{2}$, describes the torsion, and the remaining coordinates are associated with the bending deformations. The deformation
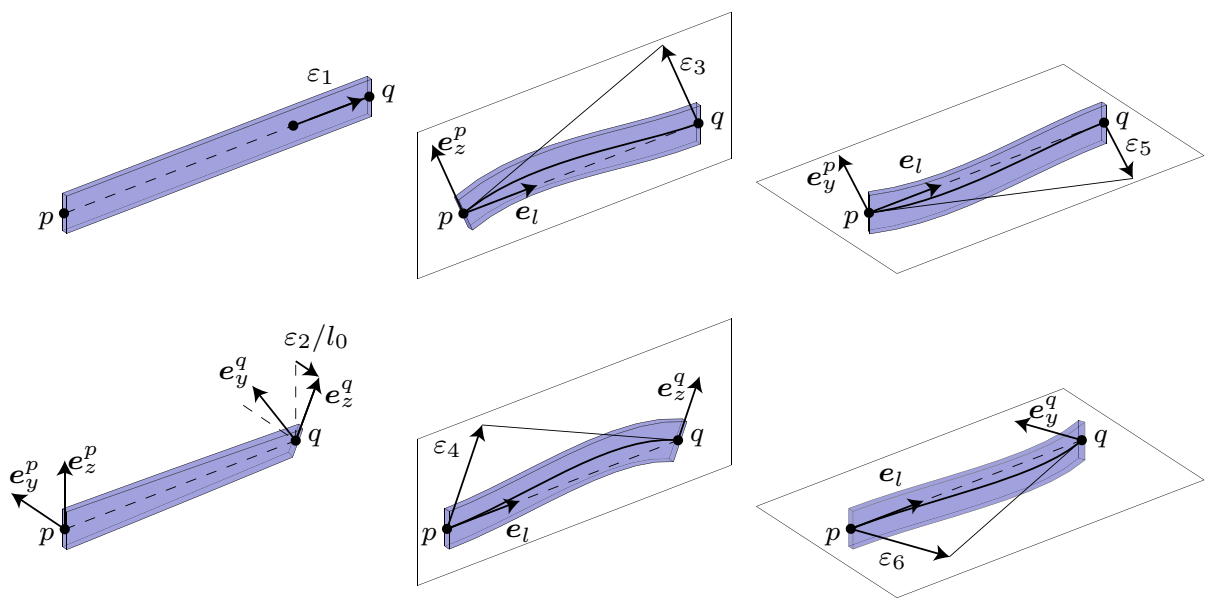

Figure 6.2: Graphical representation of the deformation modes with deformation coordinates $\varepsilon_{1}$ through $\varepsilon_{6}$. 
modes are visualized in Fig. 6.2. The definitions of the deformation coordinates are invariant under arbitrary rigid body motion of the beam element.

The derivation of the element stiffness matrix is based on a discretization of the elastic line of a three-dimensional Timoshenko beam model in a local co-rotational frame. With this formulation, large deflection problems can be solved accurately as long as the element size is chosen sufficiently small, such that the deformation coordinates of Eq. (6.2b) remain small and linear relations can be used to compute stress resultants [38].

\subsubsection{Equations of motion}

A compliant mechanism can be built up with finite elements by letting them have nodal points in common. The assemblage of finite elements is realized by defining a vector $\boldsymbol{x}$ of nodal coordinates for the entire mechanism. The deformation functions of the elements can be described in terms of the components of the vector $\boldsymbol{x}$ yielding the non-linear vector function

$$
\varepsilon=\mathcal{D}(\boldsymbol{x})
$$

which represents the basic equations for the kinematic analysis. Kinematic constraints can be introduced by putting conditions on the nodal coordinates $\boldsymbol{x}$ as well as by imposing conditions on the deformation coordinates $\varepsilon$ which are all assumed to be holonomic. For instance, rigidity of the elements can be enforced by imposing zero conditions on the subset of deformation coordinates associated with these elements, denoted by $\varepsilon^{(0)}=\mathcal{D}^{(0)}(\boldsymbol{x})=\mathbf{0}$.

An important notion in the kinematic and dynamic analysis of mechanisms is that of degrees of freedom. The number of kinematic degrees of freedom is the smallest number of coordinates that describe, together with the kinematic constraints, the configuration of the mechanism. We call them independent generalized coordinates which can be relative deformation coordinates, denoted by $\varepsilon^{(m)}$, or absolute nodal coordinates, denoted by $\boldsymbol{x}^{(m)}$. The objective of kinematic analysis is then to solve Eq. (6.3) for the vector of independent generalized coordinates

$$
\boldsymbol{q}=\left\{\begin{array}{l}
\boldsymbol{\varepsilon}^{(m)} \\
\boldsymbol{x}^{(m)}
\end{array}\right\} .
$$

The solution is expressed by means of geometric transfer functions $\mathcal{F}$ and $\mathcal{E}$ as

$$
\boldsymbol{x}=\mathcal{F}(\boldsymbol{q}), \quad \varepsilon=\mathcal{E}(\boldsymbol{q}) .
$$

The nodal velocities $\dot{\boldsymbol{x}}$ and accelerations $\ddot{\boldsymbol{x}}$ can be computed from (6.5) as

$$
\begin{gathered}
\dot{\boldsymbol{x}}=\frac{\partial \mathcal{F}}{\partial \boldsymbol{q}} \dot{\boldsymbol{q}}=\mathcal{F}_{, q} \dot{\boldsymbol{q}}, \\
\ddot{\boldsymbol{x}}=\left(\frac{\partial^{2} \mathcal{F}}{\partial \boldsymbol{q}^{2}} \dot{\boldsymbol{q}}\right) \dot{\boldsymbol{q}}+\frac{\partial \mathcal{F}}{\partial \boldsymbol{q}} \ddot{\boldsymbol{q}}=\left(\mathcal{F}_{, q \boldsymbol{q}} \dot{\boldsymbol{q}}\right) \dot{\boldsymbol{q}}+\mathcal{F}_{, q} \ddot{\boldsymbol{q}}
\end{gathered}
$$


where the subscript notation ', $\boldsymbol{q}$ ' is used to denote partial differentiation with respect to the vector $\boldsymbol{q}$ and $\left(\mathcal{F}_{, \boldsymbol{q}} \dot{\boldsymbol{q}}\right) \dot{\boldsymbol{q}}$ and other similar expressions denote multiplication over the last dimension, i.e. $\mathcal{F}_{i, j k} \dot{q}_{j} \dot{q}_{k}$. The geometric transfer functions $\mathcal{F}$ and $\mathcal{E}$ and their derivatives are determined numerically in an iterative way [38].

The inertia properties of the concentrated and distributed mass of the elements are described with the aid of lumped and consistent mass matrices [38]. Let $\boldsymbol{M}_{\boldsymbol{x}}(\boldsymbol{x})$ be the global mass matrix, obtained by assembling the lumped and consistent element mass matrices, and let $\boldsymbol{f}(\boldsymbol{x}, \dot{\boldsymbol{x}}, t)$ be the vector of external nodal forces, including gravitational forces and the velocity dependent inertia forces. Furthermore, let the loading state of each element be described by a vector of stress resultants, collected in the assembled vector $\boldsymbol{\sigma}$. This vector is dual to the vector of deformation coordinates $\boldsymbol{\varepsilon}$. Then, according to the principle of virtual work we obtain for the equations of motion of the mechanism

$$
\delta \boldsymbol{x}^{\mathrm{T}}\left(\boldsymbol{f}-\boldsymbol{M}_{\boldsymbol{x}} \ddot{\boldsymbol{x}}\right)=\delta \boldsymbol{\varepsilon}^{\mathrm{T}} \boldsymbol{\sigma}
$$

for all virtual variations $\delta \boldsymbol{x}$ and $\delta \varepsilon$ which satisfy the instantaneous kinematic constraints

$$
\delta \boldsymbol{x}=\mathcal{F}_{, \boldsymbol{q}} \delta \boldsymbol{q} \quad \text { and } \quad \delta \varepsilon=\mathcal{E}_{, \boldsymbol{q}} \delta \boldsymbol{q} .
$$

Substituting Eqs. (6.6) and (6.8) in Eq. (6.7) gives

$$
\delta \boldsymbol{q}^{\mathrm{T}} \boldsymbol{M}_{\boldsymbol{q}} \ddot{\boldsymbol{q}}=\delta \boldsymbol{q}^{\mathrm{T}}\left(\mathcal{F}_{, \boldsymbol{q}}^{\mathrm{T}}\left(\boldsymbol{f}-\boldsymbol{M}_{\boldsymbol{x}}\left(\mathcal{F}_{, \boldsymbol{q} q} \dot{\boldsymbol{q}}\right) \dot{\boldsymbol{q}}\right)-\mathcal{E}_{, \boldsymbol{q}}^{\mathrm{T}} \boldsymbol{\sigma}\right)
$$

with

$$
M_{\boldsymbol{q}}=\mathcal{F}_{, \boldsymbol{q}}^{\mathrm{T}} \boldsymbol{M}_{\boldsymbol{x}} \mathcal{F}_{, \boldsymbol{q}}
$$

Since the components of the vector $\delta \boldsymbol{q}$ are independent, we obtain the equations of motion

$$
\boldsymbol{M}_{\boldsymbol{q}} \ddot{\boldsymbol{q}}=\mathcal{F}_{, \boldsymbol{q}}^{\mathrm{T}}\left(\boldsymbol{f}-\boldsymbol{M}_{\boldsymbol{x}}\left(\mathcal{F}_{, \boldsymbol{q} q} \dot{\boldsymbol{q}}\right) \dot{\boldsymbol{q}}\right)-\mathcal{E}_{, \boldsymbol{q}}^{\mathrm{T}} \boldsymbol{\sigma}
$$

expressed as a set of non-linear second order ordinary differential equations.

\subsection{Reduction of degrees of freedom}

The equations of motion (6.11) need to be time-integrated to obtain the time response of the compliant mechanism. Reducing the number of degrees of freedom by removing high frequency vibrational modes, will result in faster simulation times. Specifically explicit time-integrators benefit from the removal of high frequency vibrational modes as larger time-steps can be taken while still providing a stable solution. We will exemplify the cause of these high frequency modes and propose two approaches to suppress them: a fixed basis method (FBM) and an interpolated basis method (IBM).

\subsubsection{Motivation and approach}

In Fig. 6.3, two examples are shown to illustrate the cause of higher frequency vibrational modes which are not of interest to obtain the desired simulation results. In Fig. 6.3(a), a simply supported beam is considered where we would like 


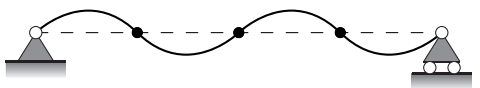

High frequency bending mode

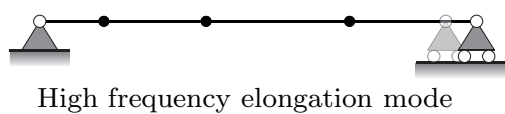

(a) Simply supported beam

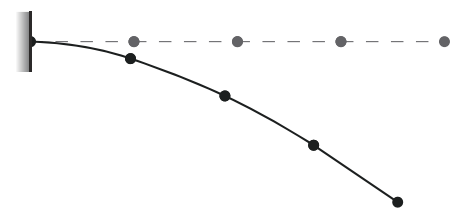

(b) Clamped beam

Figure 6.3: Examples of models that require multiple finite elements for accurate results: describing high frequency modes of a simply supported beam (a) and large deflections of a clamped beam (b).

to accurately model the illustrated high frequency bending mode. This requires a minimum of four elements as is shown. However, the model can now also be excited in the elongation mode as is shown in the figure. This elongation mode has in general a much higher eigenfrequency than the illustrated bending mode. In the second example shown in Fig. 6.3(b), a clamped beam is considered that undergoes large deflection. For an accurate representation that captures the geometric non-linear behaviour, one element is not enough as the small deformation assumption would be violated. Therefore, more elements are necessary to ensure small deformations in a co-rotational context. This again introduces high frequency vibrational modes.

In both cases the computational efficiency can be significantly increased if the high frequency modes which are not of importance for obtaining the desired simulation results, are suppressed. For this purpose constraint relations between the degrees of freedom of the model can be applied. In the case of the simply supported beam, the elongation mode can be suppressed by e.g. relating the elongations of the separate beam elements. In general, the constraint relations can be non-linear, i.e. configuration dependent,

$$
\boldsymbol{q}=\mathcal{G}(\boldsymbol{z}) \quad \text { with } \quad \operatorname{dim}(\boldsymbol{q})>\operatorname{dim}(\boldsymbol{z}),
$$

where $\boldsymbol{z}$ are the reduced set of coordinates associated with the constraint relations.

We can write Eq. (6.9) in terms of the reduced set of coordinates $\boldsymbol{z}$ by determining the virtual variations and the first and second time derivative of Eq. (6.12),

$$
\begin{aligned}
\delta \boldsymbol{q} & =\mathcal{G}_{, z} \delta \boldsymbol{z} \\
\dot{\boldsymbol{q}} & =\mathcal{G}_{, z} \dot{z} \\
\ddot{\boldsymbol{q}} & =\mathcal{G}_{, z} \ddot{z}+\left(\mathcal{G}_{, z z} \dot{z}\right) \dot{\boldsymbol{z}}
\end{aligned}
$$

Substituting the resulting expressions in Eq. (6.9), yields

$$
\begin{aligned}
\delta \boldsymbol{z}^{\mathrm{T}} \boldsymbol{M}_{\boldsymbol{z}} \ddot{\boldsymbol{z}}= & \delta \boldsymbol{z}^{\mathrm{T}}\left[\mathcal{F}_{, \boldsymbol{q}} \mathcal{G}_{, \boldsymbol{z}}\right]^{\mathrm{T}}\left(\boldsymbol{f}-\boldsymbol{M}_{\boldsymbol{x}}\left(\left(\mathcal{F}_{, \boldsymbol{q} q} \mathcal{G}_{, \boldsymbol{z}} \dot{\boldsymbol{z}}\right) \mathcal{G}_{, \boldsymbol{z}} \dot{\boldsymbol{z}}+\mathcal{F}_{, \boldsymbol{q}}\left(\mathcal{G}_{, \boldsymbol{z} z} \dot{\boldsymbol{z}}\right) \dot{\boldsymbol{z}}\right)\right) \\
& -\delta \boldsymbol{z}^{\mathrm{T}}\left[\mathcal{E}_{, \boldsymbol{q}} \mathcal{G}_{, \boldsymbol{z}}\right]^{\mathrm{T}} \boldsymbol{\sigma},
\end{aligned}
$$


with

$$
\boldsymbol{M}_{\boldsymbol{z}}=\left[\mathcal{F}_{, q} \mathcal{G}_{, \boldsymbol{z}}\right]^{\mathrm{T}} \boldsymbol{M}_{\boldsymbol{x}}\left[\mathcal{F}_{, q} \mathcal{G}_{, \boldsymbol{z}}\right]
$$

The components of $\delta \boldsymbol{z}$ are now independent. Therefore, we obtain for the reduced equations of motion

$$
\boldsymbol{M}_{\boldsymbol{z}} \ddot{\boldsymbol{z}}=\left[\mathcal{F}_{, q} \mathcal{G}_{, \boldsymbol{z}}\right]^{\mathrm{T}}\left(\boldsymbol{f}-\boldsymbol{M}_{\boldsymbol{x}}\left(\left(\mathcal{F}_{, q q} \mathcal{G}_{, \boldsymbol{z}} \dot{\boldsymbol{z}}\right) \mathcal{G}_{, \boldsymbol{z}} \dot{\boldsymbol{z}}+\mathcal{F}_{, \boldsymbol{q}}\left(\mathcal{G}_{, \boldsymbol{z} z} \dot{\boldsymbol{z}}\right) \dot{\boldsymbol{z}}\right)\right)-\left[\mathcal{E}_{, q} \mathcal{G}_{, \boldsymbol{z}}\right]^{\mathrm{T}} \boldsymbol{\sigma} .
$$

Next the FBM and the IBM are outlined which result in linear and non-linear constraint relations, respectively.

\subsubsection{Fixed basis method (FBM)}

\section{Constraint relations and derivatives}

For the fixed basis method we use a projection matrix $\boldsymbol{V}$ to project the vector of independent generalized coordinates $\boldsymbol{q}$ onto a lower dimensional subspace, i.e. the standard approach of model reduction by projection as it is commonly applied to linear systems by means of e.g. modal or Krylov subspace based methods. In this paper, the projection matrix is determined by truncating a set of eigenvectors, obtained from a modal analysis of the mechanism, such that the higher frequency vibrational modes are suppressed. With this projection matrix we can write the constraint relations of Eq. (6.12) as

$$
\boldsymbol{q}=\mathcal{G}(\boldsymbol{z})=\boldsymbol{q}_{0}+\boldsymbol{V} \boldsymbol{\eta} \quad \text { with } \quad \boldsymbol{z}=\boldsymbol{\eta} .
$$

Here $\boldsymbol{q}_{0}$ corresponds to the initial conditions of the independent generalized coordinates. For this method, the vector of reduced coordinates $\boldsymbol{z}$ consists solely of the modal coordinates $\boldsymbol{\eta}$ associated with the truncated set of eigenvectors. The first and second derivatives of $\mathcal{G}(\boldsymbol{z})$ are then

$$
\mathcal{G}_{, \boldsymbol{z}}=\boldsymbol{V} \quad \text { and } \quad \mathcal{G}_{, \boldsymbol{z} z}=\mathbf{0}
$$

which can be used in Eq. (6.13) to evaluate the reduced equations of motion.

\section{Choice of coordinates to reduce}

In general, as is mentioned by Brüls et al. [18], the dimensionality of the subspace should be rather high in order to capture geometric non-linear effects. In order to obtain a lower dimensional subspace, we propose to reduce only coordinates that remain small during simulation and are therefore suitable to be reduced with linear projection methods. To that end, the independent generalized coordinates $\boldsymbol{q}$ should be chosen accordingly. The relative deformation coordinates $\varepsilon$ of Eq. (6.3) are a viable candidate to be reduced in this way. These have to remain small for the non-linear beam elements because of the small deformation assumption. Therefore, we propose to use only these coordinates as independent generalized coordinates for this method. Equation (6.4) then becomes

$$
\boldsymbol{q}=\varepsilon^{(m)} .
$$


Note that in general, the relative deformation coordinates do not have to remain small for some commonly used elements in a multibody model. For example, the hinge element can have a large relative rotation. For models that contain such elements, the method can be adapted such that e.g. large relative rotation coordinates are not reduced as is demonstrated in [14] (see Section 5.3.2).

\section{Choice of base vectors}

The performance of the FBM depends highly on the choice of base vectors to form the projection matrix $\boldsymbol{V}$. The choice which modes to retain after a modal analysis is not a trivial one. Particularly, the phenomena known as mode-crossing and mode-veering can complicate the selection of modes. These phenomena occur when the dynamical properties of a mechanism change with a change in configuration. This is often the case for large-stroke compliant mechanisms whose stiffness properties in supporting directions are highly configuration dependent.

Mode-crossing occurs when the eigenfrequencies of two different modes cross as the configuration changes. Assuming that the eigenvectors are sorted according to their eigenfrequencies for every considered configuration, any eigenvectors with crossing eigenfrequencies will change order at a mode-crossing point.

Mode-veering occurs when the eigenfrequencies of two different modes approach each other and then veer away without crossing as the configuration changes. The eigenvectors of these two modes after the veering point will be swapped. During veering, the two modes will continuously, but abruptly, transform from one into the other by a rotation of the eigenvectors [15].

In both cases, when modes of interest are crossing or veering with other modes, the crossing and veering modes need to be included in the projection matrix $\boldsymbol{V}$ to ensure that the lower frequency dynamics over the full range of motion is correctly retained. Also, the configuration dependency of eigenvectors further away from mode-veering or crossing points needs to be taken into account when determining the projection matrix $\boldsymbol{V}$.

A systematic approach to determine a projection matrix that is capable of correctly describing the first $r$ eigenvectors in the operating range of the mechanism is outlined by the following procedure:

1. In the operating range of the mechanism, determine in $n$ equidistant equilibrium reference configurations the linearized equations of motion [42].

2. Compute the matrix $\boldsymbol{V}^{(i)}$ with $i=1,2, \ldots, n$, by performing a modal analysis of the linearized equations of motion at each equilibrium reference configuration and retaining the first $r$ normalized eigenvectors corresponding to the vibrational modes of interest.

3. for $j=1,2, \ldots, r$ do

4. Form the matrix $\boldsymbol{W}^{(j)}$ according to

$$
\boldsymbol{W}^{(j)}=\left[\boldsymbol{V}_{j}^{(1)}, \boldsymbol{V}_{j}^{(2)}, \ldots, \boldsymbol{V}_{j}^{(n)}\right]
$$


where $\boldsymbol{W}^{(j)}$ is a matrix containing the $j^{\text {th }}$ normalized mode for all $n$ reference configurations. This matrix has

$$
\operatorname{rank}\left(\boldsymbol{W}^{(j)}\right) \geq 1
$$

and this rank is guaranteed to be greater than one if the $j^{\text {th }}$ mode is crossing or veering with other modes or if this mode is highly configuration dependent.

5. Perform an $\mathrm{SVD}^{1}$ of the matrix $\boldsymbol{W}^{(j)}$ to determine a set of base vectors that can accurately describe the $j^{\text {th }}$ mode in all $n$ reference configurations.

6. From the SVD analysis we retain the set of left singular vectors with large corresponding singular values, yielding the matrix $\boldsymbol{U}^{(j)}$.

\section{7. end for}

8. Concatenate the matrices $\boldsymbol{U}^{(j)}$ with $j=1,2, \ldots, r$ and form

$$
\boldsymbol{Q}=\left[\boldsymbol{U}^{(1)}, \boldsymbol{U}^{(2)}, \ldots, \boldsymbol{U}^{(r)}\right]
$$

9. Finally, perform an SVD of the matrix $\boldsymbol{Q}$ to determine a set of base vectors that can describe the modes of interest in all $n$ reference configurations. The projection matrix $\boldsymbol{V}$ of Eq. (6.16) is then obtained by retaining the singular vectors with large corresponding singular values as in steps 5 and 6 .

In the first step, the equilibrium reference configurations are chosen to be obtained equidistantly to ensure that no configuration bias is introduced in the SVD analysis of steps 5 and 6 . Also, to avoid bias, only normalized eigenvectors, or alternatively eigenvectors with the same length, should be computed in step 2 .

The last step is used to remove any linear dependency of the vectors in the matrix $\boldsymbol{Q}$ and to possibly obtain a smaller set of base vectors for the projection matrix $\boldsymbol{V}$. This step is particularly important when modes of interest are crossing or veering as this can result in a strong linear dependency between the matrices $\boldsymbol{U}^{(j)}$.

We note that an approach with one SVD analysis is also possible by directly performing the SVD analysis on all modes of interest in all reference configurations [14] (as is done in Section 5.3.3). However, by performing an SVD analysis on each mode of interest separately, we find it is easier to determine which singular vectors to keep in order to ensure that the first $r$ modes of interest are correctly preserved over the full range of motion.

Note that the procedure described in this section is not guaranteed to give a set of base vectors in which no high frequency modes are present because

$$
\operatorname{rank}(\boldsymbol{V}) \geq r
$$

${ }^{1}$ The SVD of a matrix $\boldsymbol{A}$ is given by: $\boldsymbol{A}=\boldsymbol{U} \boldsymbol{\Sigma} \boldsymbol{V}^{\mathrm{T}}$, where $\boldsymbol{\Sigma}$ is a diagonal matrix of singular values and $\boldsymbol{U}$ and $\boldsymbol{V}$ are the matrices with the left and right singular vectors, respectively. 
due to the fact that the rank of the subspace required to describe each mode separately is greater or equal to one, see Eq. (6.20). This makes it likely that the rank of $\boldsymbol{V}$ is greater than $r$ and thus some higher frequency modes are necessary to describe the configuration dependency of the vibrational modes of interest.

\subsubsection{Interpolated basis method (IBM)}

For the realization of a reduction method that does not need additional vectors to describe the configuration dependency of the vibrational modes of interest, an interpolation strategy can be employed. For this purpose, the subspace spanned by the reduced set of eigenvectors over the operating range of the mechanism can be interpolated. We will refer to this method as the interpolated basis method.

\section{Constraint relations and derivatives}

To start, the independent generalized coordinates are written as

$$
\boldsymbol{q}=\mathcal{G}(\boldsymbol{z})=\mathcal{Q}(s)+\mathcal{V}(s) \boldsymbol{\eta}+\boldsymbol{q}_{0} \quad \text { with } \quad \boldsymbol{z}=\left\{\begin{array}{c}
\boldsymbol{s} \\
\boldsymbol{\eta}
\end{array}\right\}
$$

Here, $\mathcal{Q}(s)$ represents the gross reference motion of the compliant mechanism with respect to the initial conditions $\boldsymbol{q}_{0}$ and $\mathcal{V}(\boldsymbol{s})$ is the subspace spanned by a reduced set of eigenvectors at a particular configuration described by the configuration coordinates $s$. The configuration coordinates should be chosen such that they adequately represent the gross reference motion. In this paper we restrict ourselves to a single configuration coordinate $s$. This single configuration coordinate then describes the main trajectory of motion in three dimensional space. The first and second derivatives of $\mathcal{G}(\boldsymbol{z})$ to its reduced coordinates are

$$
\begin{aligned}
\mathcal{G}_{, s} & =\mathcal{Q}_{, s}(s)+\mathcal{V}_{, s}(s) \boldsymbol{\eta}, \\
\mathcal{G}_{, \boldsymbol{\eta}} & =\mathcal{V}(s) \\
\mathcal{G}_{, s s} & =\mathcal{Q}_{, s s}(s)+\mathcal{V}_{, s s}(s) \boldsymbol{\eta}, \\
\mathcal{G}_{, s \boldsymbol{\eta}} & =\mathcal{V}_{, s}(s), \\
\mathcal{G}_{, \boldsymbol{\eta} \boldsymbol{\eta}} & =\mathbf{0}
\end{aligned}
$$

Equation (6.24) can be used to construct $\mathcal{G}_{, \boldsymbol{z}}$ and $\mathcal{G}_{, \boldsymbol{z z}}$, which are necessary in Eq. (6.15).

\section{Generation of interpolation data}

Expressions for $\mathcal{Q}(s), \mathcal{V}(s)$ and their derivatives are obtained by interpolation. The interpolation data are generated by determining in $n$ reference configurations the difference between the independent generalized coordinates and the initial conditions $\boldsymbol{q}_{0}$, yielding $\Delta \boldsymbol{q}^{(i)}$, and the first $r$ orthonormalized eigenvectors $\boldsymbol{V}^{(i)}$ corresponding to the vibrational modes of interest at interpolation points $s^{(i)}$ along 
the main trajectory of motion. Here the superscript $(i)$ denotes the $i^{\text {th }}$ reference configuration.

The eigenvectors in the matrix $\boldsymbol{V}^{(i)}$ are obtained from a modal analysis of the linearized equations of motion. The modal analysis is performed while fixing the configuration coordinate $s$ for every reference configuration, such that the eigenvectors contained in the matrix $\boldsymbol{V}^{(i)}$ will have no components along the main trajectory of motion $^{2}$.

\section{Interpolation of the reference configurations}

An expression for $\mathcal{Q}(s)$ is obtained by interpolating $\Delta \boldsymbol{q}^{(i)}$ with $i=1,2, \ldots, n$, along the main trajectory of motion. For this interpolation we choose to use piecewise third order polynomial splines through the interpolation points $s^{(i)}$. The interpolation coefficients are chosen such that the first and second derivatives are continuous at the interpolation points.

\section{Interpolation of modal subspaces}

Interpolating the eigenvectors $\boldsymbol{V}^{(i)}$ with $i=1,2, \ldots, n$, along the main trajectory of motion is not guaranteed to give satisfactory results for $\mathcal{V}(s)$. In $[18,33]$ modecrossing and mode-veering are named as the cause for these interpolation difficulties of eigenvectors. Interpolating over a mode-crossing or veering point will introduce large gradients in $\mathcal{V}_{s}(s)$ of Eq. (6.24) if the eigenvectors in $\boldsymbol{V}^{(i)}$ are sorted according to their eigenfrequencies. This results in large numerical errors or more often cause the time-integration of the equations of motion to fail.

To cope with mode-crossing and veering, it is proposed in $[5,18]$ to use modetracking algorithms based on the modal assurance criterion (MAC) [26]. This method is very effective in locating and sorting modes that cross and it is able to track modes that veer, provided that the spatial discretization is sufficiently dense. However, large gradients in $\mathcal{V}_{, s}(s)$ can still be found for modes that veer, due to the swift rotation of the eigenvectors. Nonetheless, the subspace spanned by these rotating eigenvectors changes only a little. A better approach is then to determine base vectors that do not change rapidly over mode-veering regions, as is suggested in [33]. To achieve this, we propose a modal subspace interpolation (MSI) algorithm, outlined by the following steps:

1. Orthonormalize the $r$ retained eigenvectors in the $n$ reference configurations, resulting in $\boldsymbol{V}^{(i)}$ for the $i^{\text {th }}$ reference configuration.

2. Set $\boldsymbol{W}^{(1)}=\boldsymbol{V}^{(1)}$ to define the starting configuration.

3. for $i=1,2, \ldots, n-1$ do

${ }^{2}$ This is to ensure that the matrix $\mathcal{G}_{, z}$ is of full rank as the first column of this matrix, Eq.(6.24a), already describes the change of the configuration of the mechanism in the direction of motion. 
4. Compute $\hat{\boldsymbol{W}}^{(i+1)}$ by projecting the base vectors of $\boldsymbol{W}^{(i)}$ onto the subspace spanned by $\boldsymbol{V}^{(i+1)}$,

$$
\hat{\boldsymbol{W}}^{(i+1)}=\boldsymbol{V}^{(i+1)} \boldsymbol{V}^{(i+1) \mathrm{T}} \boldsymbol{W}^{(i)} .
$$

5. Compute $\boldsymbol{W}^{(i+1)}$ by normalizing the columns of $\hat{\boldsymbol{W}}^{(i+1)}$.

\section{6. end for}

7. Obtain $\overline{\boldsymbol{W}}^{(i)}$ by mass normalizing the base vectors of $\boldsymbol{W}^{(i)}$ with $i=1,2, \ldots, n$. For the $j^{\text {th }}$ vector at the $i^{\text {th }}$ configuration, this is

$$
\overline{\boldsymbol{W}}_{j}^{(i)}=\frac{\boldsymbol{W}_{j}^{(i)}}{\boldsymbol{W}_{j}^{(i) \mathrm{T}} \boldsymbol{M}_{\boldsymbol{q}}^{(i)} \boldsymbol{W}_{j}^{(i)}},
$$

where $\boldsymbol{M}_{\boldsymbol{q}}^{(i)}$ is the linearized mass matrix at the $i^{\text {th }}$ configuration.

8. Obtain $\mathcal{V}(s)$ by interpolating the matrices $\overline{\boldsymbol{W}}^{(i)}$ with $i=1,2, \ldots, n$, along the main trajectory of motion.

In steps 3 through 6 it is ensured that when the mechanisms is moving through a veering region, the projected base vectors of $\boldsymbol{W}^{(i+1)}$ change only a little with respect to $\boldsymbol{W}^{(i)}$. Still, $\boldsymbol{W}^{(i+1)}$ represents the exact same subspace as $\boldsymbol{V}^{(i+1)}$. This is illustrated in Fig. 6.4 for a two dimensional subspace in $\mathbb{R}^{3}$. Note that both veering modes have to be present in the subspace for this to work effectively. The same applies for a mode of interest that crosses other modes if no precautions are taken. In this case, all modes that cross the mode of interest need to be retained. However, the modes can be sorted with a MAC based mode-tracking method prior to the first step of the algorithm. This makes it possible to include only the mode of interest without having to add any of the crossing modes.

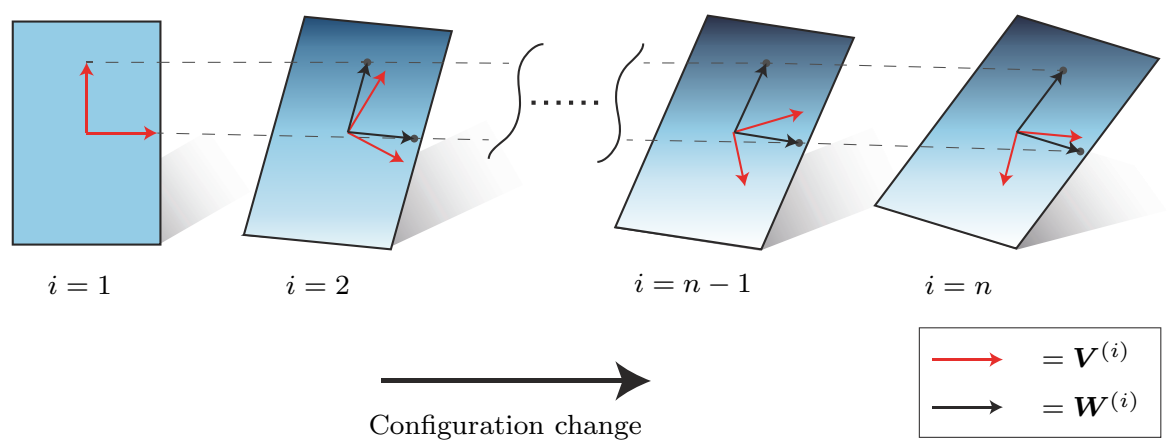

Figure 6.4: Illustration of steps 3 through 6 of the MSI algorithm, for a change of a two dimensional subspace in $\mathbb{R}^{3}$, e.g. during mode-veering. The projected base vectors $\boldsymbol{W}^{(i+1)}$ change only a little with respect to $\boldsymbol{W}^{(i)}$, but span the same subspace as $\boldsymbol{V}^{(i+1)}$. 
Step 7 is not strictly necessary. However, we observed smoother varying base vectors when their length is mass normalized. The gradients in $\mathcal{V}_{, s}(s)$ are then smaller, which is beneficial for the time-integration of the equations of motion.

For the interpolation in step 8 we use piecewise third order polynomial splines similar to the interpolation of the reference configurations to obtain $\mathcal{Q}(s)$.

\subsection{Application example}

As an application example to test the effectiveness of the FBM and the IBM, we consider a spatial model of a large stroke compliant straight guidance in a gravity field. The results are compared to simulation results of an unreduced model. For the IBM, we also consider a case where a mode-tracking algorithm is implemented instead of the MSI algorithm described in Section 6.3.3. For the time-integration of the equations of motion we use an explicit variable step-size integrator, specifically the Shampine-Gordon integrator [63].

\subsubsection{Compliant straight guidance model}

The compliant straight guidance shown in Fig. 6.5, consists of two flexures connected by a rigid intermediate body. Each of the flexures are modelled with five non-linear

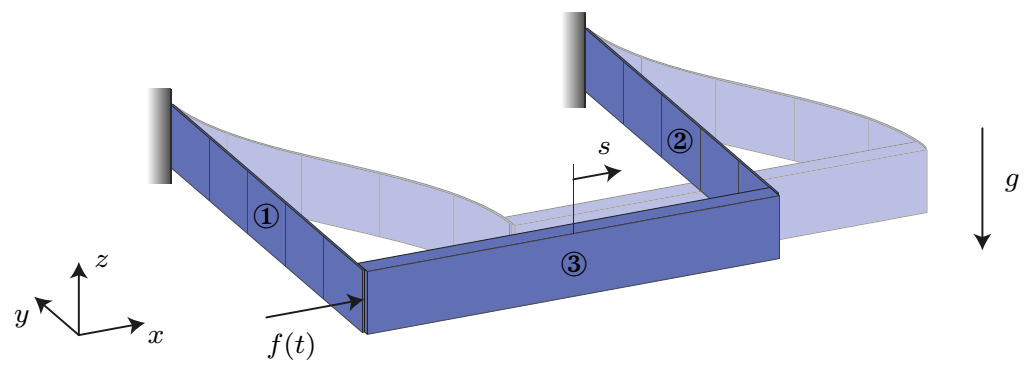

Figure 6.5: Spatial straight guidance model in a gravity field $g$, where the flexures (1) and (2) are modelled with 5 beam elements each and the intermediate body (3) is modelled as a rigid element. The maximal deflected state is shown in a lighter colour.

Table 6.1: Spatial straight guidance model parameters.

\begin{tabular}{lll}
\hline Property & Flexures (1) (2) & Rigid beam (3) \\
\hline & & \\
Length, $l$ & $0.2 \mathrm{~m}$ & $0.2 \mathrm{~m}$ \\
Height, $h$ & $30 \cdot 10^{-3} \mathrm{~m}$ & $30 \cdot 10^{-3} \mathrm{~m}$ \\
Width, $w$ & $1 \cdot 10^{-3} \mathrm{~m}$ & $30 \cdot 10^{-3} \mathrm{~m}$ \\
Young's Modulus, $E$ & $2.1 \cdot 10^{11} \mathrm{~N} / \mathrm{m}^{2}$ & \\
Density, $\rho$ & $7600 \mathrm{~kg} / \mathrm{m}^{3}$ & $7600 \mathrm{~kg} / \mathrm{m}^{3}$ \\
\hline
\end{tabular}


spatial beam elements. Each beam element in the flexures suppresses the elongation mode but allows deformations of the other five deformation modes, see Fig. 6.2. This results in a model with a total of 44 degrees of freedom. The physical properties and dimensions of the flexures and the rigid intermediate body are given in Tab. 6.1. The model contains no damping. Furthermore, gravity is included and is acting in the negative $z$-direction, where $g=9.81 \mathrm{~m} / \mathrm{s}^{2}$. The model is excited by a force as a function of time

$$
f(t)= \begin{cases}\frac{F_{\max }}{2}(1-\cos (20 \pi t)), & 0 \mathrm{~s} \leq t \leq 0.1 \mathrm{~s}, \\ 0, & t>0.1 \mathrm{~s},\end{cases}
$$

where the maximal applied force $F_{\max }=100 \mathrm{~N}$. The resulting maximal deflection is then $0.0834 \mathrm{~m}$, which is about $41.7 \%$ of the flexure lengths.

We determine in the initial configuration, the equilibrium configuration including gravity. This configuration is used to obtain the initial conditions for the timeresponse simulations of the unreduced and reduced models. We simulate the time response of the unreduced model and compute the eigenfrequencies over the range of motion of the straight guidance. This simulation will serve as a benchmark for the reduced models. Of particular interest for the reduced models is the displacement caused by gravity in the $z$-direction measured at the coordinate $s$. Furthermore, we will investigate how well the reduced models are able to reproduce the eigenfrequencies of the unreduced model over the full range of motion. We will determine what is required for the reduced models to accurately represent the first two eigenfrequencies and as a second case, the first four eigenfrequencies. The second case is included because from a control perspective usually the first few eigenmodes are of interest for e.g. stability analysis. All eigenfrequency data are presented as a function of the $x$-displacement of the rigid intermediate body. These are obtained for the time instances between the dashed lines of Fig. 6.6 where the time response of the rigid intermediate body in $x$-direction is shown.

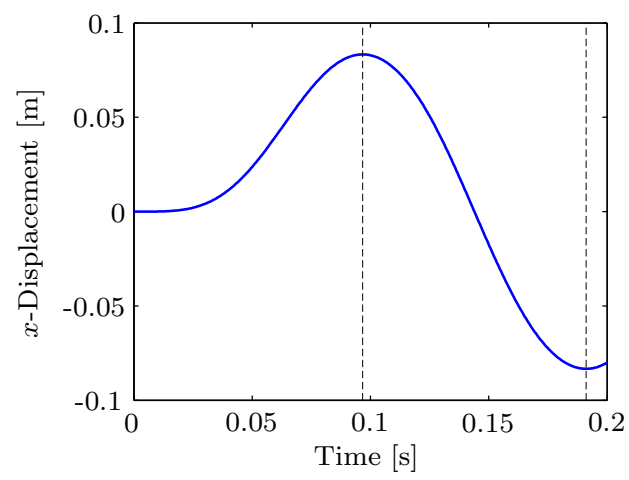

Figure 6.6: Time response of the rigid intermediate body in $x$-direction. 
To determine the effect of the reduction on the computational efficiency, we will compare the number of function evaluations the integrator requires to complete the simulation for the different reduced models.

The considered reduced models for the FBM and IBM are obtained without gravity to see if they are robust for small deviations from the main trajectory of motion.

\subsubsection{Reduction with fixed basis method}

For the reduction with the FBM, we choose the 44 independent generalized coordinates to be a subset of the deformation mode coordinates of the beam elements in the flexures. Specifically, a subset of the torsion and the bending related coordinates of Eq. (6.2b) are chosen, as all elongation modes are suppressed.

\section{Generating the reduced models}

For the first case, where we aim to describe the first two eigenfrequencies over the full range of motion, we consider two FBM reduced models. The first reduced model is obtained by doing a modal analysis in the equilibrium configuration of the compliant mechanism. The first two eigenvectors are used as base vectors for the projection matrix of this reduced model, and it will be referred to as the MODAL-2 reduced model. The second reduced model is obtained by following the procedure in Section 6.3.2, where we considered 300 linearized models evenly spaced in the range of motion of the compliant mechanism.

In Fig. 6.7 the singular values, with respect to the sum of singular values of all singular vectors, are shown for the first and second vibrational mode of the 300

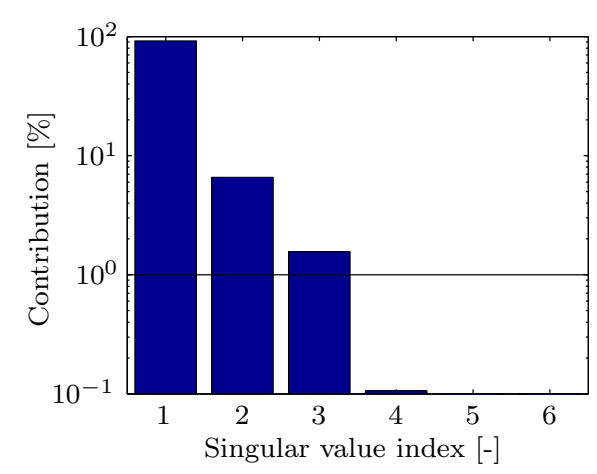

(a) SVD analysis of the first mode

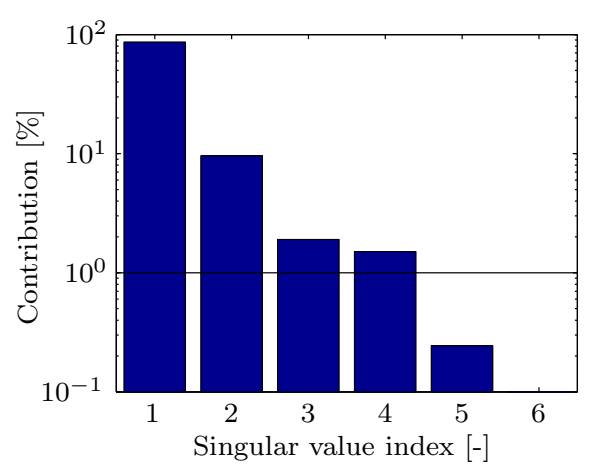

(b) SVD analysis of the second mode

Figure 6.7: Percentage of singular values with respect to the sum of singular values, for the singular vectors obtained from an SVD of the first mode (a) and of the second mode (b) for all considered configurations. The horizontal line indicates $1 \%$ contribution of a singular vector. 


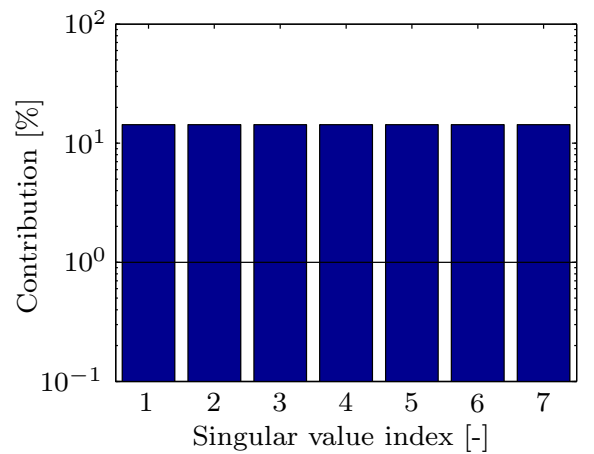

(a) SVD-7 model

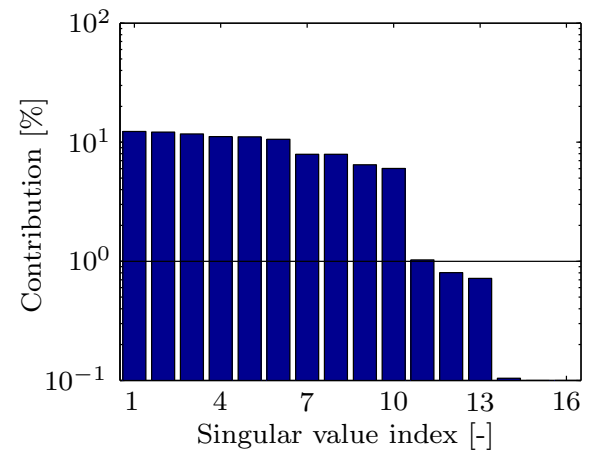

(b) SVD-13 model

Figure 6.8: Percentage of singular values with respect to the sum of singular values, for the singular vectors considered for the SVD-7 reduced model (a) and for the SVD-13 reduced model (b). The horizontal line indicates $1 \%$ contribution of a singular vector.

linearized models. Singular vectors with a contribution greater than $1 \%$ are retained, resulting in a collection of seven singular vectors. The $1 \%$ criterion showed good results, but should by no means be taken for a rule. Following the procedure in Section 6.3.2, the collection of seven singular vectors is subjected to another SVD, yielding the results shown in Fig 6.8(a). In this case, all seven singular vectors are required to describe the first and second vibrational mode over the full range of motion, yielding an SVD-7 reduced model.

For the second case, where the first four eigenfrequencies are of interest over the full range of motion, an SVD-13 reduced model with thirteen singular vectors is considered. This model is obtained in the same way as the SVD-7 reduced model. For the SVD-13 reduced model we retained four and five singular vectors from the SVD of the third and fourth vibrational modes of the 300 considered linearized models. This results in a collection of sixteen singular vectors by adding the seven singular vectors of the first two vibrational modes. These are again subjected to an SVD to remove any linear dependencies, yielding the results shown in Fig 6.8(b). From this result we determined the thirteen singular vectors of the SVD-13 reduced model. Here we note that the last three singular vectors have a smaller contribution than the first ten. However, these singular vectors proved important for preserving the configuration dependency of the third and fourth vibrational mode over the range of motion.

\section{Simulation results}

In Fig. 6.9 the first and second eigenfrequencies of the unreduced and the three reduced models are shown as a function of the coordinate $s$ which describes the $x$-displacement of the rigid intermediate body. The first vibrational mode is a translational mode in the $x$-direction, while the second vibrational mode corresponds to an out-of-plane 


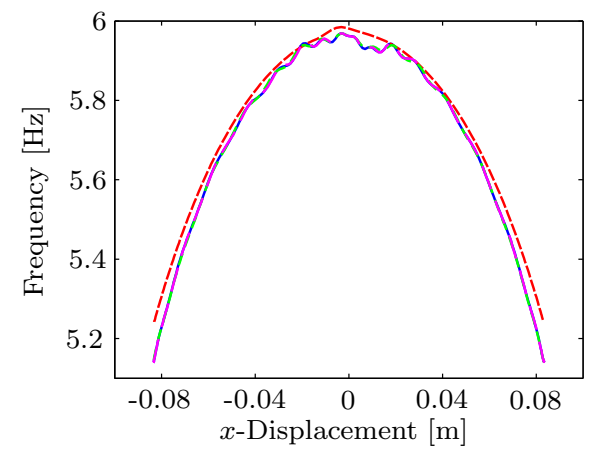

(a) First eigenfrequency

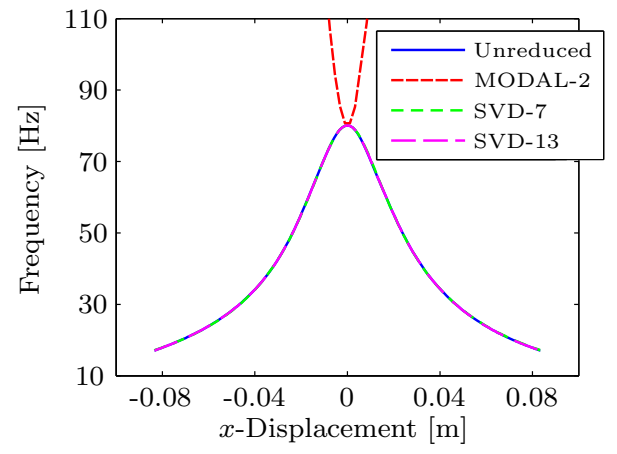

(b) Second eigenfrequency

Figure 6.9: First (a) and the second (b) eigenfrequencies of the compliant straight guidance as a function of the $x$-displacement of the rigid intermediate body: comparison of the unreduced and the FBM reduced models.

bending mode, i.e. deflection in the $z$-direction. The MODAL-2 model is able to correctly predict the first eigenfrequency as the compliant mechanism is deflected, see Fig. 6.9(a). The second eigenfrequency of the MODAL-2 model, shown in Fig. 6.9(b), rises to $1100 \mathrm{~Hz}$ when the compliant mechanism is maximally deflected. Whereas, due to geometric non-linearities, this eigenfrequency should drastically drop when deflected. It is clear from these results that simply retaining the first two eigenvectors obtained in the equilibrium configuration of the compliant mechanism is not sufficient to obtain correct results over the full range of motion. In contrast, the SVD-7 and SVD-13 models give very good results for the first and second eigenfrequencies.

The third and fourth eigenfrequencies of the unreduced model and the SVD-13 reduced model are shown in Fig. 6.10. The corresponding vibrational modes are internal bending modes of the flexures. The SVD-13 reduced model is able to accurately describe the third and the fourth eigenfrequencies over the full range of motion, as is intended by the procedure described in Section 6.3.2.

In Fig. 6.11, the $z$-displacements of the rigid intermediate body measured at the coordinate $s$, see Fig. 6.5, for the unreduced and reduced models are shown as a function of time. For an accurate time-response in this direction it is important that the second vibrational mode is correctly estimated as this is the first bending mode in this direction. The MODAL-2 model is not able to correctly estimate this mode over the full range of motion, resulting in the poor performance observed in Fig. 6.11. The SVD-7 and SVD-13 reduced models again give very good results.

In Tab. 6.2 the number of function evaluations required by the integrator to complete the simulation and the highest occurring eigenfrequencies are given for the considered models. The unreduced model requires 1060595 function evaluations. The reason for the unreduced model to require so many function evaluations is due to the inclusion of gravity. Even though we start close to the equilibrium position by 


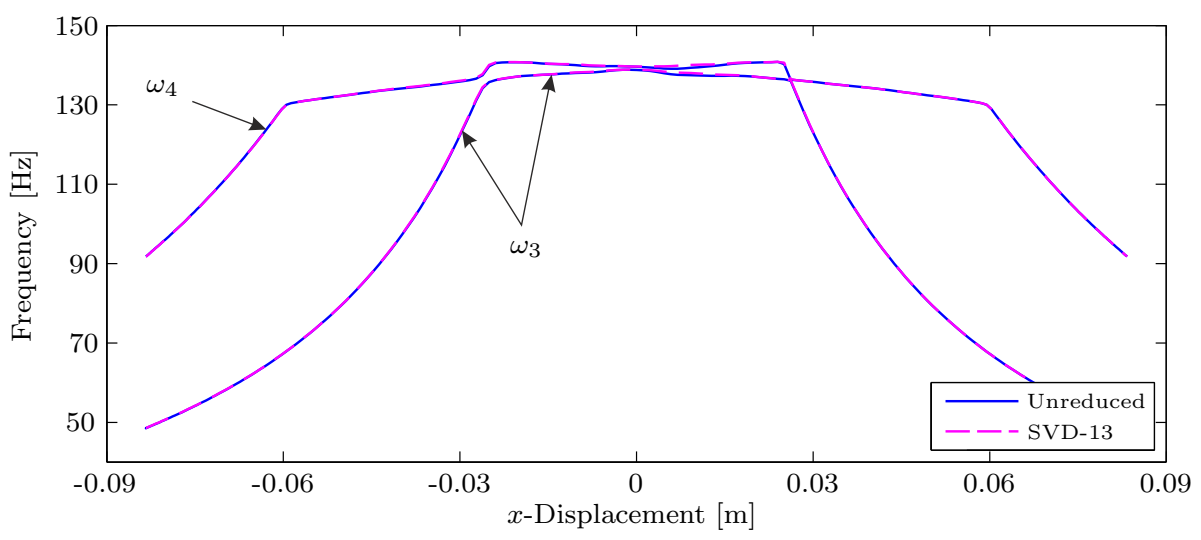

Figure 6.10: Third $\left(\omega_{3}\right)$ and fourth $\left(\omega_{4}\right)$ eigenfrequencies of the compliant straight guidance as a function of the $x$-displacement of the rigid intermediate body: comparison of the unreduced and the FBM reduced models.

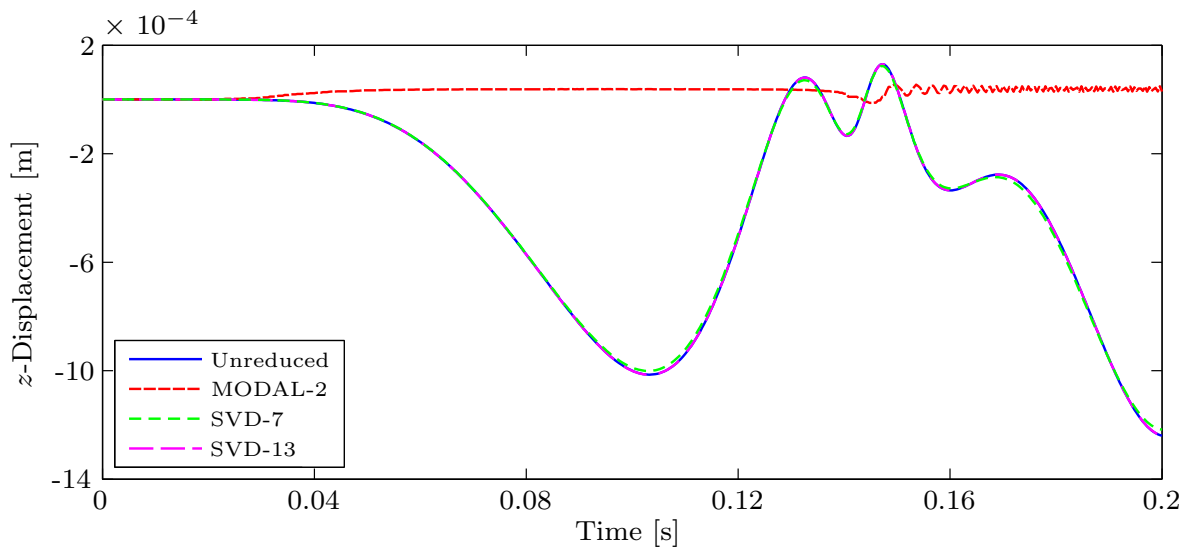

Figure 6.11: $z$-Displacement of the rigid intermediate body measured at coordinate $s$ as a function of time: comparison of the unreduced and the FBM reduced models.

adjusting the initial conditions, some small deviations are enough to excite higher vibrational modes in particular stiff modes in the $z$-direction. As no damping is present in the example, they will remain excited for the duration of the simulation. In contrast, an unreduced simulation without gravity only needs about 30000 function evaluations.

A reduction of the number of function evaluations is realized for all reduced models. The MODAL-2 reduced model is the quickest, though gives incorrect 
Table 6.2: Function evaluations and highest occurring eigenfrequencies of the unreduced and reduced models obtained with the fixed basis method.

\begin{tabular}{lllll}
\hline & Unreduced & MODAL-2 & SVD-7 & SVD-13 \\
\hline & & & & \\
Function evaluations [-] & 1060595 & 1647 & 12246 & 87804 \\
Highest occurring eigenfrequency $[\mathrm{Hz}]$ & 193050 & 1097 & 7181 & 18076 \\
\hline
\end{tabular}

results as previously mentioned. In the case of the SVD-7 reduced model, additional modes are necessary with a maximum eigenfrequency of about $7200 \mathrm{~Hz}$ to be able to describe the first two eigenfrequencies over the full range of motion. A similar effect is observed for the SVD-13 reduced model. Here, modes with eigenfrequencies up to $18000 \mathrm{~Hz}$ are required to be able to describe the first four eigenfrequencies. The necessary inclusion of these high frequency modes, which are well outside the frequency range of interest, is a considerable drawback of the method.

\subsubsection{Reduction with interpolated basis method}

We considered the use of both relative and absolute coordinates as the 44 independent generalized coordinates for the reduction with the IBM. From a theoretical point of view, both sets of coordinates should be valid to be reduced with the IBM. However, when using relative coordinates we observed some numerical issues which are most likely caused by a buildup of small errors that can result in a slightly different configuration than the unreduced model. Hence, the results presented here are obtained using absolute nodal coordinates for the independent generalized coordinates.

\section{Generating the reduced models}

The reduced models are generated from 300 linearized models, evenly spaced in the range of motion of the compliant mechanism. As is mentioned in Section 6.4.1, these models are obtained in the absence of gravity to determine if the reduced models are robust for small deviations from the main trajectory of motion.

To be able to accurately describe the first two eigenfrequencies over the full range of motion, an interpolated two degrees of freedom model, MSI-2, is considered. The first degree of freedom is associated with the gross reference motion, while the second degree of freedom describes the out-of-plane bending mode previously mentioned in Section 6.4.2. The MSI-2 model is obtained by interpolating the reference configurations and the modal subspaces according to the MSI algorithm. The use of the MSI algorithm is not strictly necessary for this case, as the first two modes do not cross or veer with any other modes.

To obtain a reduced model capable of describing the first four eigenfrequencies over the full range of motion, we note that five different vibration modes are veering and crossing with the modes associated with the third and fourth eigenfrequencies. 
As all these modes need to be included in the modal subspace to describe these eigenfrequencies over the full range of motion, we consider an MSI-7 reduced model obtained with the MSI algorithm. Here, the first degree of freedom again describes the gross reference motion, while the remaining degrees of freedom are associated with the interpolated modal subspace. Additionally, we will consider a reduced model where the interpolation of the modal subspace is realized by interpolating the six eigenvectors at each interpolating point, after sorting the eigenvectors with a MAC based mode-tracking algorithm as described in [18]. This model is referred to as the MAC-7 reduced model.

\section{Simulation results}

The first and second eigenfrequencies of the unreduced and the reduced models are shown in Fig. 6.12. From Fig. 6.12 it can be seen that the MSI-2 model is able to accurately predict the first and second eigenfrequency. For the MAC-7 model, the first eigenfrequency has two deviating peaks near $\pm 0.06 \mathrm{~m}$ deflection. This behaviour is caused by numerical inaccuracies due to large time derivatives of the reduced coordinates as the model is transitioning through a mode-veering region, see Fig. 6.13. In contrast, the MSI-7 reduced model is able to correctly predict the first eigenfrequency. Good matches for the second eigenfrequency are found for all the IBM reduced models.

In Fig. 6.13, the third and the fourth eigenfrequencies of the unreduced model and of the MSI-7 and MAC-7 reduced models are shown. Both models give quite good results for the prediction of these eigenfrequencies, though some slight deviations can be observed. These deviations become less if the linearized models from which the reference configurations and the modal subspaces are derived, are

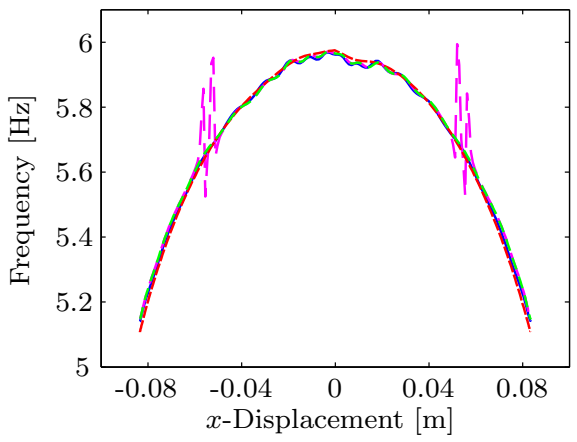

(a) First eigenfrequency

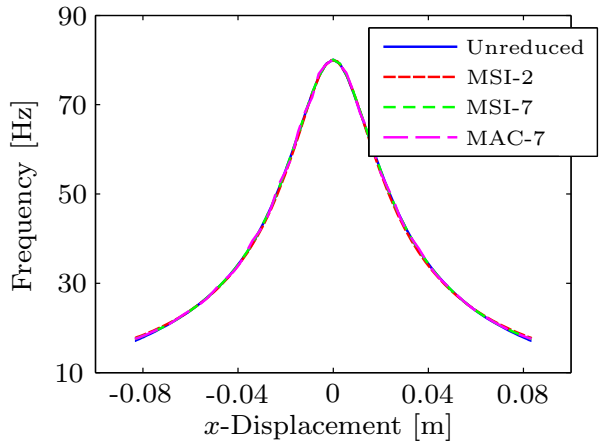

(b) Second eigenfrequency

Figure 6.12: First (a) and the second (b) eigenfrequencies of the compliant straight guidance as a function of the $x$-displacement of the rigid intermediate body: comparison of the unreduced and the IBM reduced models. 


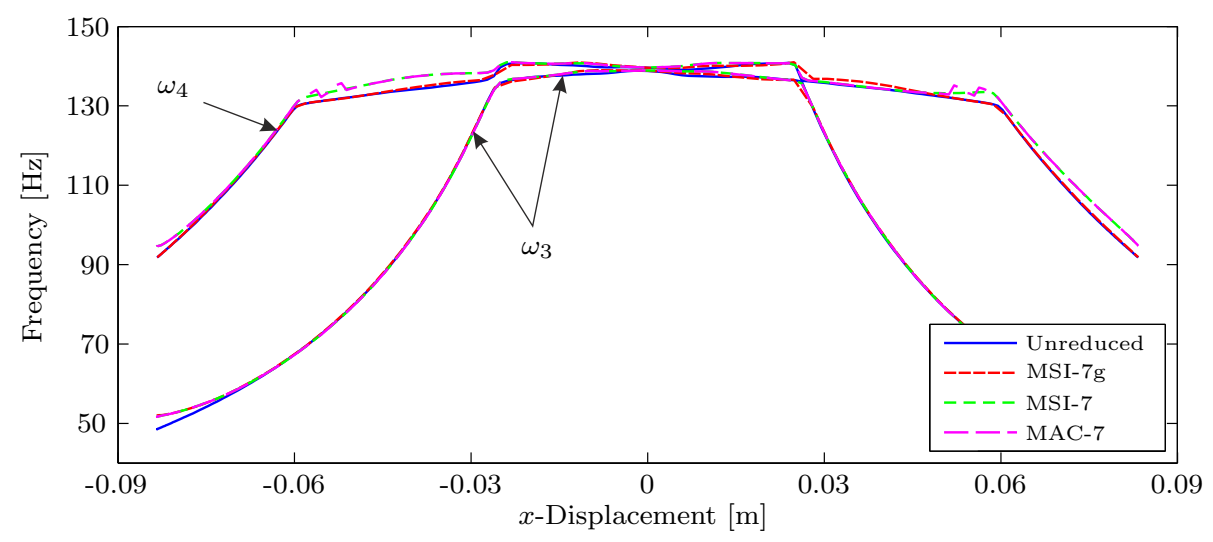

Figure 6.13: Third $\left(\omega_{3}\right)$ and fourth $\left(\omega_{4}\right)$ eigenfrequencies of the compliant straight guidance as a function of the $x$-displacement of the rigid intermediate body: comparison of the unreduced and the IBM reduced models.

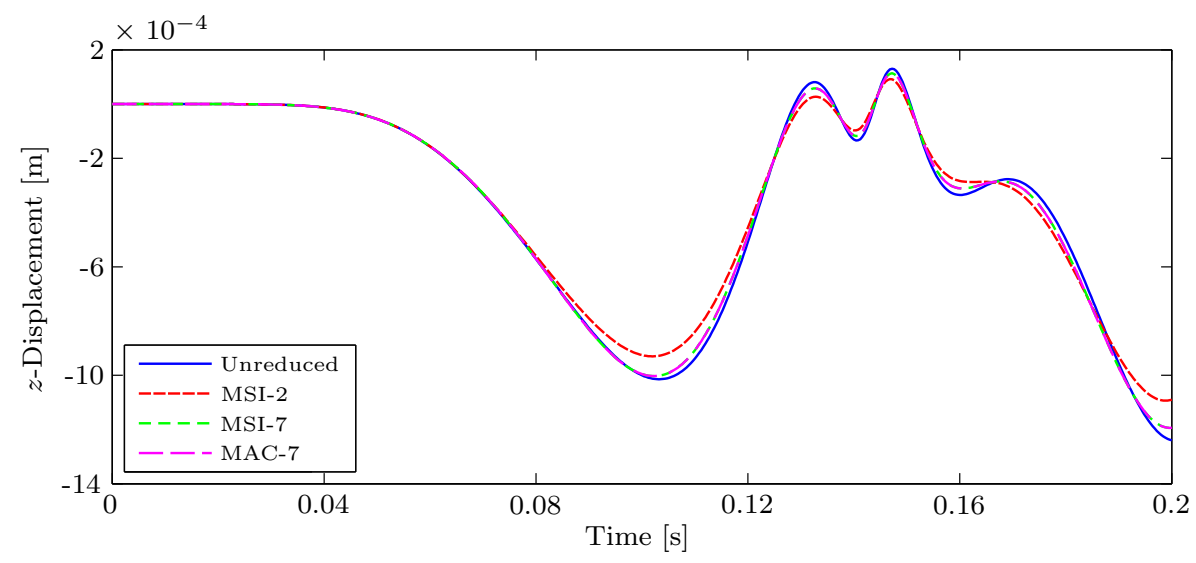

Figure 6.14: $z$-Displacement of the rigid intermediate body measured at coordinate $s$ as a function of time: comparison of the unreduced and the IBM reduced models.

computed considering gravity. This is demonstrated by the results of the MSI-7g reduced model, which is obtained considering gravity at each of the reference configurations. Some small peaks of the fourth eigenfrequency as in Fig. 6.12(a), are observed for the MAC-7 reduced model, although the effect is less pronounced than for the first eigenfrequency.

From Fig. 6.14 it can be seen that all considered IBM reduced models are capable of 
predicting the $z$-displacement of the rigid intermediate body quite accurately. Though the results obtained with the MSI-2 reduced model are slightly stiffer.

In Tab. 6.3 the number of function evaluations required by the integrator to complete the simulation and the highest occurring eigenfrequencies are given for the considered models. It is clear that a significant reduction in the number of function evaluations can be realized for the interpolated basis method. The benefit of using the MSI algorithm compared to a mode-tracking algorithm, with respect to computational efficiency, is also evident. The MAC-7 reduced model requires significantly more function evaluations near mode-veering regions than the MSI-7 reduced model, resulting in the observed difference in function evaluations.

Table 6.3: Function evaluations and highest occurring eigenfrequencies of the unreduced and reduced models obtained with the interpolated basis method.

\begin{tabular}{lllll}
\hline & & & & \\
& Unreduced & MSI-2 & MSI-7 & MAC-7 \\
\hline & & & & \\
Function evaluations [-] & 1060595 & 626 & 947 & 1486 \\
Highest occurring eigenfrequency $[\mathrm{Hz}]$ & 193050 & 80 & 379 & 380 \\
\hline
\end{tabular}

\subsubsection{Comparison of the fixed and interpolated basis method}

From the presented results, we can conclude that accurate results can be obtained with both methods. We observe that more accurate reduced models of lower order can be realized with the interpolated basis method than with the fixed basis method. For example, the MSI-2 reduced model is able to predict the second eigenfrequency accurately, while poor results are observed for the MODAL-2 reduced model. The reduced models obtained with the interpolated basis method are also considerably cheaper to solve than those obtained with the fixed basis method. On the other hand, the fixed basis method is easier to implement as no second order derivatives are required in its derivation, unlike the interpolated basis method. This effect will be more pronounced if more than one configuration coordinate is considered to describe the gross reference motion of a large-stroke compliant mechanism.

\subsection{Conclusions}

In this paper a reduction method is proposed for the efficient time-integration of flexible multibody models. The approach is based on constraining the independent generalized coordinates that define the configuration of the multibody model. Two different implementations for constraining the independent generalized coordinates are considered, a fixed basis method and an interpolated basis method.

For the fixed basis method, linear constraints are applied to independent generalized coordinates that remain small during simulation. The projection of the independent coordinates to a lower dimensional subspace effectively suppresses 
the excitation of high frequency modes, whereas geometric non-linear effects are retained in the geometric transfer functions. We propose an SVD based procedure to determine an adequate set of base vectors for the projection matrix. The obtained set of base vectors are such that the vibrational modes of interest can accurately be described in the operating range of the mechanism.

For the interpolated basis method, non-linear constraints are applied to the independent generalized coordinates. The independent generalized coordinates are described as a superposition of the gross reference motion and the vibrational modes of interest. Here the gross reference motion is obtained by interpolating reference configurations throughout the trajectory of motion. Furthermore, the subspace spanned by the vibrational modes of interest is interpolated using a modal subspace interpolation algorithm. The algorithm constructs base vectors that span the modal subspace, which vary as little as possible with a change of configuration. This allows accurate interpolation of the modal subspaces without having to resort to mode-tracking algorithms to deal with mode-crossing and mode-veering phenomena.

The effectiveness of both methods is demonstrated by considering a flexible multibody model of a compliant straight guidance that undergoes large deflection. From simulation results of this example it is observed that accurate reduced models can be obtained with both methods. The fixed basis method still requires the inclusion of some high frequency modes to be able to describe the configuration dependency of the vibrational modes of interest. This makes the fixed basis method less computationally efficient than the interpolated basis method. An increase in accuracy and computational efficiency for the interpolation basis method is observed when using the modal subspace interpolation algorithm opposed to interpolating eigenvectors sorted by a mode-tracking algorithm.

Acknowledgements This research is financially supported by the Dutch association Point-One, project MOV-ET PNE08006, of the Dutch Department of Economic Affairs, Agriculture and Innovation. The authors acknowledge the contributions of Derk ten Hoopen for the initial investigation of a variety of model reduction approaches. 



\section{Analysis and simulation of a large-stroke compliant mechanism}

The interpolated basis method (IBM) of the previous chapter is applied to obtain a reduced model of the two degree of freedom large-stroke compliant positioning mechanism of Chapter 4. In Chapter 6, the IBM is set up for systems where the configuration is described with only one coordinate. To be able to apply this method to the two degree of freedom compliant mechanism, the gross motion of the mechanism needs to be defined as a function of a single parameter. This can be achieved by prescribing the desired path of the end-effector. It is investigated how to obtain the proper base vectors for the IBM along this predetermined path. Two different approaches are investigated. In the first approach, in accordance with the theory of Chapter 6, eigenvectors are obtained by constraining the direction of the path, i.e. the constrained eigenvectors. In the second approach, the use of unconstrained eigenvectors is considered instead. Both approaches are applied to obtain reduced models of the compliant mechanism. Subsequently, these models are evaluated in equilibrium positions along the path and their eigenfrequencies are compared to those of the unreduced model. To conclude, a closed-loop time-response simulation of the mechanism is performed.

\subsection{Model reduction along a predetermined path}

The fixed basis method (FBM) of Chapter 6 can be applied straightforwardly to reduce models of compliant mechanisms with multiple degrees of freedom ${ }^{1}$. However, unavoidably in the FBM, unwanted high frequency vibrational modes remain in the reduced model. Therefore, the application of the IBM is preferred in most cases. The IBM is designed such that a single coordinate defines the gross reference motion along which the modal subspace and reference configurations are interpolated. For a single degree of freedom compliant mechanism, it is straightforward to assign a coordinate

\footnotetext{
${ }^{1}$ In this context, in line with Chapter 1 , the mention of 'degree of freedom' in relation to a compliant mechanism refers to the intended mobility of that mechanism and should not be confused with the number of degrees of freedom of the mechanism model.
} 
to describe the gross reference motion as it can only be actuated in one direction. This is however not the case for a mechanism that has multiple degrees of freedom, such as the two degree of freedom compliant mechanism of Chapter 4. To apply the IBM to such a mechanism, a path needs to be defined that depends on a single parameter and along which the reference configurations and the modal subspaces can be interpolated. The reference configurations can be determined straightforwardly as is described in Chapter 6, whereas the following subsections outline the determination of the modal subspaces for the interpolation along this path.

\subsubsection{Applying the IBM for mechanisms with multiple degrees of freedom}

For a proper IBM reduced model, the Jacobian matrix $\mathcal{G}_{, z}$ of Eq. (6.13a) needs to be full rank. This matrix relates the possible variations of the independent generalized coordinates $\delta \boldsymbol{q}$ (the degrees of freedom of the model) with the variations of the reduced set of coordinates $\delta \boldsymbol{z}$. Hence, the columns of $\mathcal{G}_{, \boldsymbol{z}}$ represent the subspace that defines in which directions the configuration of the mechanism is allowed to change. Therefore, to be able to describe the first few vibrational modes of interest of a compliant mechanism, the columns of the matrix $\mathcal{G}_{, z}$ should describe the subspace spanned by the corresponding eigenvectors.

For the IBM, this matrix can be represented with Eq. (6.24), as

$$
\mathcal{G}_{, \boldsymbol{z}}=\left[\begin{array}{cc}
\mathcal{Q}_{, s}(s)+\mathcal{V}_{, s}(s) \boldsymbol{\eta}, & \mathcal{V}(s)
\end{array}\right],
$$

where $\mathcal{Q}(s)$ represents the gross reference motion, $\mathcal{V}(s)$ is the interpolated modal subspace with an associated set of modal coordinates $\boldsymbol{\eta}$ and $s$ is the configuration coordinate describing the position of the end-effector along a predetermined path. Furthermore, the reduced coordinates $\boldsymbol{z}$, defined in Eq. (6.23), consist of the configuration coordinate $s$ and the modal coordinates $\boldsymbol{\eta}$.

For the special case when the mechanism is exactly following the reference motion, i.e. $\boldsymbol{\eta}=\mathbf{0}$, Eq. (7.1) simplifies to

$$
\left.\mathcal{G}_{, \boldsymbol{z}}\right|_{\boldsymbol{\eta}=\mathbf{0}}=\left[\mathcal{Q}_{, s}(s), \mathcal{V}(s)\right] .
$$

Here $\mathcal{Q}_{s}(s)$ describes how the configuration of the mechanism is changing along the path. For a properly designed single degree of freedom compliant mechanism, the first vibrational mode should match the change of the configuration in the direction of motion. In other words, $\mathcal{Q}_{s}(s)$ corresponds to the first vibrational mode of a single degree of freedom compliant mechanism, whereas $\mathcal{V}(s)$ represents the remaining vibrational modes of interest. Therefore, to obtain a matrix $\mathcal{G}_{, \boldsymbol{z}}$ with full rank, the first eigenvector should not be included in the interpolated modal subspace $\mathcal{V}(s)$. To achieve this, the coordinate $s$ can be fixed in every reference configuration when determining the eigenvectors for the computation of $\mathcal{V}(s)$, as is suggested in Section 6.3.3. None of the resulting constrained eigenvectors then describes the first vibrational mode of the mechanism as the motion tangential to the path is constrained. 
However, for a mechanism that is designed to have $m$ degrees of freedom, the coordinate $s$ that defines the path is not necessarily an actual coordinate of the model and can therefore not straightforwardly be constrained to obtain the constrained eigenvectors. Alternatively, the use of unconstrained eigenvectors can be considered. For a single degree of freedom compliant mechanism, the first unconstrained eigenvector can simply be omitted in the interpolation of the modal subspace as it matches the gross reference motion. Applying the same strategy for an $m$ degree of freedom compliant mechanism is however inadequate. This is because it is not guaranteed for such a mechanism that the first unconstrained eigenvector describes the change of the configuration in the direction of the path. Instead, a linear combination of the first $m$ eigenvectors typically describes the change of the configuration.

Procedures for a correct derivation of respectively the constrained and unconstrained eigenvectors, are explained in the following subsections.

\subsubsection{Constrained eigenvectors}

Let $\mathcal{P}(s)$ be the vector function defining the path of the end-effector of the mechanism as a function of the coordinate $s$ which represents the distance travelled along the path. The number of coordinates that define the path has to be equal to the $m$ degrees of freedom of the mechanism. These path coordinates can be defined as a subset of the absolute nodal coordinates $\boldsymbol{x}$ or relative coordinates $\boldsymbol{\varepsilon}$ of Eq.(6.3). However, for determining the constrained eigenvectors, they must be chosen to be part of the set of independent generalized coordinates $\boldsymbol{q}$ of Eq.(6.4). Then, the vector function $\mathcal{P}(s)$ and its first derivative $\mathcal{P}_{, s}(s)$ yield

$$
\begin{aligned}
\boldsymbol{q}_{p} & =\mathcal{P}(s), \\
\boldsymbol{q}_{p, s} & =\mathcal{P}_{, s}(s),
\end{aligned}
$$

where $\boldsymbol{q}_{p}$ is an $m \times 1$ vector of path coordinates and is a subset of the $k \times 1$ vector $\boldsymbol{q}$. Furthermore, $\boldsymbol{q}_{p, s}$ is the $m \times 1$ vector defining the direction of the path at the location $s$ and is a subset of the $k \times 1$ vector $\boldsymbol{q}_{s}$. The vector $\boldsymbol{q}_{s}$ is zero for the independent generalized coordinates that are not associated with the path. Computing the nullspace of $\boldsymbol{q}_{s}^{\mathrm{T}}$ yields

$$
\boldsymbol{P}=\operatorname{Null}\left(\boldsymbol{q}_{, s}^{\mathrm{T}}\right),
$$

where the columns of the $k \times(k-1)$ matrix $\boldsymbol{P}$ are independent and perpendicular to $\boldsymbol{q}_{s}$. In other words, the columns of $\boldsymbol{P}$ represent the subspace in which no motion is allowed in the direction of the path.

Next, the linearized equations of motion in a configuration of the mechanism at rest and in equilibrium $(\dot{\boldsymbol{q}}=\mathbf{0}, \ddot{\boldsymbol{q}}=\mathbf{0})$ at the location $s$ along the path, are determined (see Appendix E). From Eq.(E.6) the mass matrix $\boldsymbol{M}_{\boldsymbol{q}}$, the structural stiffness matrix $\boldsymbol{K}_{\boldsymbol{q}}$ and the geometric stiffness matrix $\boldsymbol{G}_{\boldsymbol{q}}$ are obtained. The constrained eigenvectors are then determined by solving the generalized eigenvalue problem

$$
\boldsymbol{P}^{\mathrm{T}}\left(\boldsymbol{K}_{\boldsymbol{q}}+\boldsymbol{G}_{\boldsymbol{q}}-\omega_{j}^{2} \boldsymbol{M}_{\boldsymbol{q}}\right) \boldsymbol{P} \boldsymbol{v}_{j}=\mathbf{0} \quad \text { with } \quad j=1,2, \ldots, k-1
$$


where the $(k-1) \times 1$ vector $\boldsymbol{v}_{j}$ corresponds to the $j^{\text {th }}$ constrained eigenvector with eigenfrequency $\omega_{j}$. Collecting the first $r$ eigenvectors of interest and pre-multiplying them with the matrix $\boldsymbol{P}$ yields the $k \times r$ matrix $\boldsymbol{V}$ of constrained eigenvectors. By repeating the process for the $n$ discrete locations $s^{(i)}$ along the path, the set of matrices $\boldsymbol{V}^{(i)}$ with $i=1,2, \ldots, n$, is obtained. Next, the matrices $\boldsymbol{V}^{(i)}$ can be used in the modal subspace interpolation (MSI) algorithm of Section 6.3.3 to compute $\mathcal{V}(s)$.

\subsubsection{Unconstrained eigenvectors}

Typically, for a compliant mechanism with $m$ degrees of freedom, a linear combination of the first $m$ unconstrained eigenvectors should be able to describe the change of the configuration at a location $s$ in the direction of the path. As this motion is already described by $\mathcal{Q}_{s}(s)$ of Eq.(7.2), this linear combination of modes should be excluded from the interpolation of the modal subspace for obtaining $\mathcal{V}(s)$. Let the vector $\boldsymbol{\eta}_{p}$ represent this linear combination and let the matrix $\boldsymbol{V}_{p}$ represent the first $m$ unconstrained eigenvectors associated with the mechanism motion, then the vector $\boldsymbol{V}_{p} \boldsymbol{\eta}_{p}$ represents the motion in the direction of the path at a location $s$. The problem of finding a suitable set of modes for use in the interpolation of the modal subspace, then reduces to finding the set of $m-1$ base vectors $\boldsymbol{V}_{b}$ from $\boldsymbol{V}_{p}$ that are perpendicular to $\boldsymbol{V}_{p} \boldsymbol{\eta}_{p}$. The solution, assuming that the eigenvectors in $\boldsymbol{V}_{p}$ are orthonormal, is given by

$$
\boldsymbol{V}_{b}=\boldsymbol{V}_{p} \operatorname{Null}\left(\boldsymbol{\eta}_{p}^{\mathrm{T}}\right),
$$

which can be verified by checking that $\boldsymbol{V}_{b}^{\mathrm{T}}\left(\boldsymbol{V}_{p} \boldsymbol{\eta}_{p}\right)$ is the zero vector:

$$
\left(\boldsymbol{V}_{p} \operatorname{Null}\left(\boldsymbol{\eta}_{p}^{\mathrm{T}}\right)\right)^{\mathrm{T}} \boldsymbol{V}_{p} \boldsymbol{\eta}_{p}=\mathbf{0} .
$$

The dimensionality of the matrix $\boldsymbol{V}_{b}$ is $k \times(m-1)$ for a model with $k$ independent generalized coordinates $\boldsymbol{q}$.

The matrix $\boldsymbol{V}_{p}$ required in Eq. (7.6) can be obtained from the $k \times r$ matrix of unconstrained eigenvectors $\boldsymbol{V}_{u}$ :

$$
\boldsymbol{V}_{u}=\left[\boldsymbol{V}_{p}, \boldsymbol{V}_{a}\right],
$$

which is computed by performing a modal analysis on the linearized equations of motion for a configuration of the mechanism at rest and in equilibrium at a location $s$ along the path, and subsequently retaining and orthonormalizing the first $r$ eigenvectors of interest. The $k \times(r-m)$ matrix $\boldsymbol{V}_{a}$ contains additional unconstrained eigenvectors of interest that should be included for the computation of $\mathcal{V}(s)$. Appending the matrix $\boldsymbol{V}_{b}$ with $\boldsymbol{V}_{a}$, yields

$$
\boldsymbol{V}=\left[\boldsymbol{V}_{b}, \boldsymbol{V}_{a}\right] .
$$

Here, the $k \times(r-1)$ matrix $\boldsymbol{V}$ represents the modal subspace that is to be interpolated along the path to obtain $\mathcal{V}(s)$. For the computation of $\boldsymbol{V}_{b}$ in Eq.(7.6), the vector $\boldsymbol{\eta}_{p}$ still needs to be determined. This vector can be obtained from the path derivative $\mathcal{P}_{, s}(s)$ of Eq.(7.3), which is demonstrated next. 
Unlike in the previous section, the coordinates of the path $\mathcal{P}(s)$ are not required to be a subset of the $k$ independent generalized coordinates $\boldsymbol{q}$. Instead, the path coordinates can be defined by a subset of the absolute nodal coordinates $\boldsymbol{x}$ or relative deformation coordinates $\varepsilon$ of Eq.(6.3). Let these subsets be represented by $\boldsymbol{x}_{p}$ and $\boldsymbol{\varepsilon}_{p}$, then the vector function $\mathcal{P}(s)$ and its first derivative $\mathcal{P}_{, s}(s)$ yield

$$
\begin{aligned}
\left\{\begin{array}{l}
\boldsymbol{x}_{p} \\
\boldsymbol{\varepsilon}_{p}
\end{array}\right\} & =\mathcal{P}(s), \\
\left\{\begin{array}{l}
\boldsymbol{x}_{p, s} \\
\boldsymbol{\varepsilon}_{p, s}
\end{array}\right\} & =\mathcal{P}_{, s}(s),
\end{aligned}
$$

where the $m \times 1$ vector $\left\{\boldsymbol{x}_{p, s}^{\mathrm{T}}, \boldsymbol{\varepsilon}_{p, s}^{\mathrm{T}}\right\}^{\mathrm{T}}$ defines the direction of the path at the coordinate $s$. By using the first order geometric transfer functions $\mathcal{F}_{, \boldsymbol{q}}$ and $\mathcal{E}_{, \boldsymbol{q}}$ given by Eq.(6.8), the direction of the path can also be described in terms of the vector $\boldsymbol{V}_{p} \boldsymbol{\eta}_{p}$,

$$
\left\{\begin{array}{c}
\boldsymbol{x}_{p, s} \\
\boldsymbol{\varepsilon}_{p, s}
\end{array}\right\}=\left[\begin{array}{c}
\mathcal{F}_{p, \boldsymbol{q}} \\
\mathcal{E}_{p, \boldsymbol{q}}
\end{array}\right] \boldsymbol{V}_{p} \boldsymbol{\eta}_{p} .
$$

Here, the matrices $\mathcal{F}_{p, \boldsymbol{q}}$ and $\mathcal{E}_{p, \boldsymbol{q}}$ represent the rows of $\mathcal{F}_{, \boldsymbol{q}}$ and $\mathcal{E}_{, \boldsymbol{q}}$ that are associated with the path coordinates $\boldsymbol{x}_{p}$ and $\boldsymbol{\varepsilon}_{p}$, respectively. The $m \times m$ matrix

$$
\left[\begin{array}{c}
\mathcal{F}_{p, \boldsymbol{q}} \\
\mathcal{E}_{p, \boldsymbol{q}}
\end{array}\right] \boldsymbol{V}_{p}
$$

can be inverted to obtain the vector $\boldsymbol{\eta}_{p}$.

The process should be repeated at $n$ discrete locations $s^{(i)}$ along the path to obtain, with Eq.(7.9), the set of matrices $\boldsymbol{V}^{(i)}$ with $i=1,2, \ldots, n$. The matrices $\boldsymbol{V}^{(i)}$ can then be used in the MSI algorithm of Section 6.3.3 to obtain the interpolated modal subspace $\mathcal{V}(s)$.

\subsection{Model reduction of the large-stroke compliant mechanism}

In this section, the two degree of freedom large-stroke compliant positioning mechanism of Chapter 4 is reduced with the IBM using both constrained and unconstrained eigenvectors for the interpolation of the modal subspace. The eigenfrequencies obtained with the reduced models are compared with the eigenfrequency results of the unreduced SPACAR model determined in Section 4.4. Furthermore, a closed-loop time-response simulation is performed.

\subsubsection{Reduced models}

To be able to perform a closed-loop time-response simulation, 'actuators' and 'sensors' need to be defined in the model. The actuators are modelled by adding two hinge elements to the model. The hinge elements are placed between the fixed world and UpperArmX1 and UpperArm Y, respectively, see Fig. 7.1. Furthermore, the axes of rotation of the hinges are in the global $z$-direction. By applying moments to the 
hinge elements, the model can be actuated in the $x$ - and $y$-directions. The relative rotation angles of the hinges are used as sensor outputs to realize co-located control.

The model with the hinge elements is reduced with the IBM by determining the constrained and unconstrained eigenvectors in 100 equilibrium configurations along the path $\mathcal{P}(s)$ of the end-effector which is depicted in Fig. 7.1 and defined by Eq.(4.1). Here the coordinate $s$ is defined to be the distance travelled along the path. The resulting reduced models are referred to by IBM-c and IBM-u to indicate the use of constrained and unconstrained eigenvectors, respectively. In correspondence with the results presented in Chapter 4, the reduced models should preserve the vibrational modes with eigenfrequencies up to $200 \mathrm{~Hz}$. Therefore, the reduced models are designed such that the first eight eigenvectors are retained by including seven constrained or unconstrained eigenvectors in the interpolation of the modal subspace to obtain $\mathcal{V}(s)$. This yields reduced models with 8 degrees of freedom instead of the 688 degrees of freedom of the unreduced model of Chapter 4.

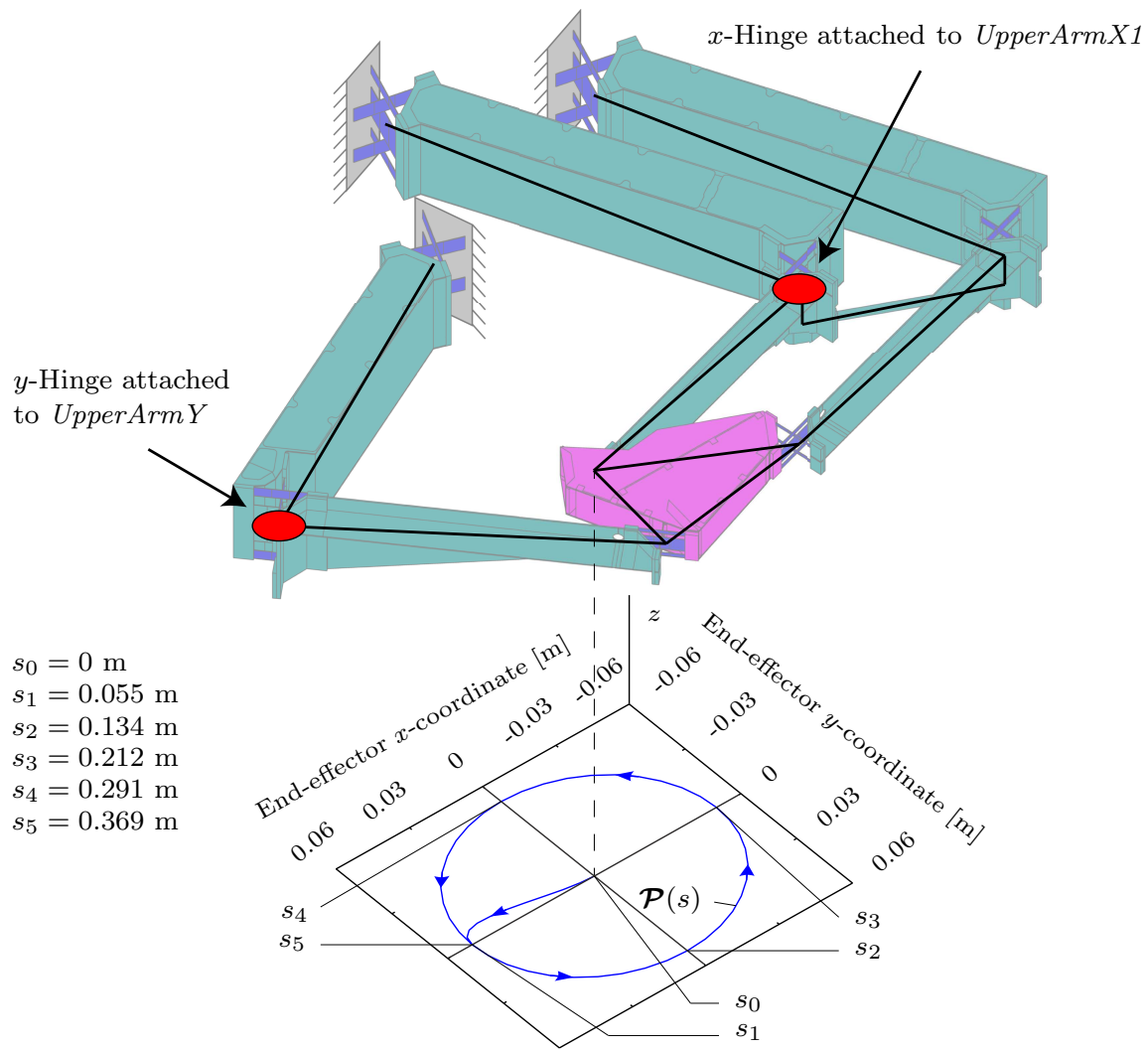

Figure 7.1: Actuator hinge element locations and the path of the end-effector. 


\subsubsection{Comparison of eigenfrequencies}

To determine the applicability of the reduced models, the first eight eigenfrequencies in the equilibrium configurations along the path are compared with results obtained with the unreduced model. The eigenfrequencies for the reduced models are computed by solving the generalized eigenvalue problem of the combined structural and geometric stiffness matrices $\boldsymbol{K}_{\boldsymbol{z}}+\boldsymbol{G}_{\boldsymbol{z}}$ and mass matrix $\boldsymbol{M}_{\boldsymbol{z}}$ of Eq.(F.7) derived in Appendix F. Figures 7.2 and 7.3 show the first eight eigenfrequencies, computed in the 100 equilibrium configurations along the path for the unreduced and the reduced models. The first four eigenfrequencies are correctly predicted by both reduced models. Some small differences between the reduced models can be observed for the fifth and sixth eigenfrequencies, whereas significant differences can be observed for the seventh and eighth eigenfrequencies. Here, the IBM-u reduced model is predicting all eight eigenfrequencies in correspondence with the unreduced model along the path, whereas deviating results are obtained for the IBM-c reduced model. By using Eq. (7.5) for the computation of the constrained eigenvectors, the mechanism is constrained such that the end-effector can not move in the direction of the path. Adding such a constraint changes the dynamics of the model. Therefore, it is not guaranteed with the combination of the seven constrained eigenvectors and $\mathcal{Q}_{s}(s)$ of Eq. (7.2), that all the original eight unconstrained eigenvectors can be recovered. Obviously, by adding more constrained eigenvectors, the IBM-c model gains more freedom to correctly describe the simpler vibrational modes with lower eigenfrequencies. This explains why the IBM-c model is capable of correctly predicting the first five eigenfrequencies along the path, whereas increasingly larger errors are observed for the higher eigenfrequencies.

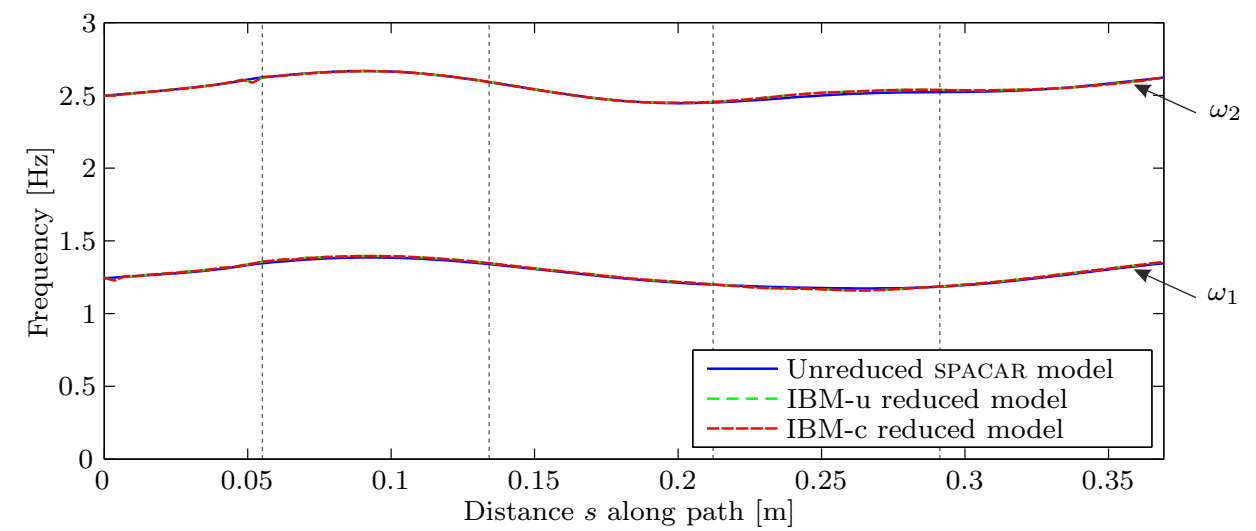

Figure 7.2: The first two eigenfrequencies $\omega_{1}$ and $\omega_{2}$ of the two degree of freedom large-stroke compliant mechanism as functions of the distance along the path for the unreduced and reduced models. The vertical dashed lines correspond to the locations $s_{1}$ through $s_{4}$ shown in Fig. 7.1. 


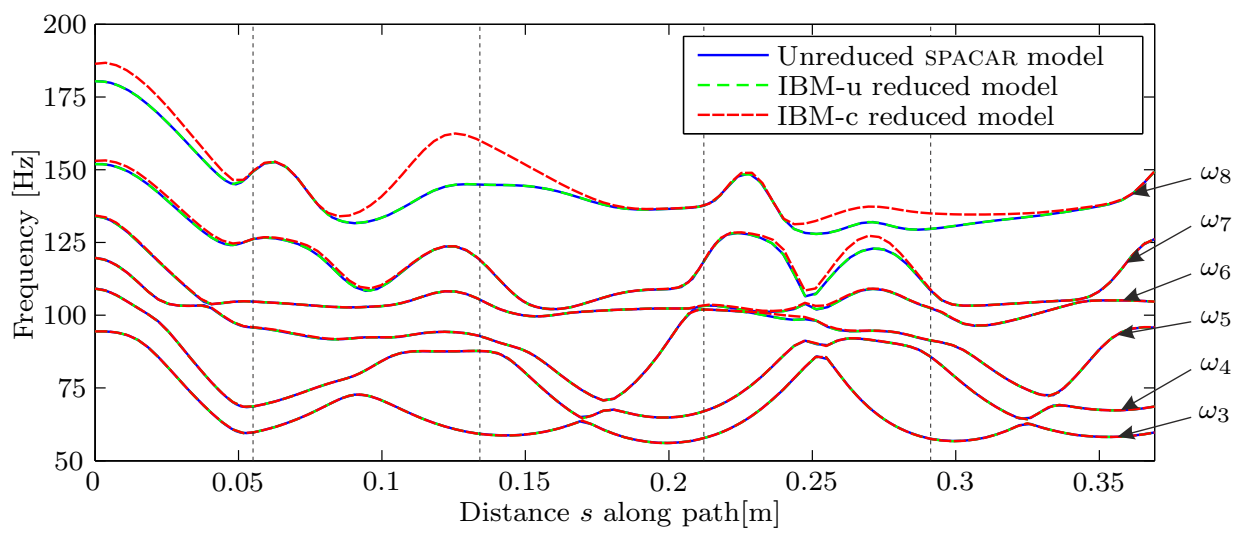

Figure 7.3: Eigenfrequencies $\omega_{3}$ through $\omega_{8}$ of the two degree of freedom large-stroke compliant mechanism as functions of the distance along the path for the unreduced and reduced models. The vertical dashed lines correspond to the locations $s_{1}$ through $s_{4}$ shown in Fig. 7.1.

\subsubsection{Closed-loop time-response simulation}

\section{Controller parameters}

Two proportional-derivative (PD) controllers with single inputs and outputs are used to actuate the mechanism ${ }^{2}$. The controller outputs are then given by

$$
\begin{aligned}
& M_{x}(t)=K_{x}^{p}\left(\phi_{x}^{\mathrm{ref}}(t)-\phi_{x}(t)\right)+K_{x}^{d}\left(\dot{\phi}_{x}^{\mathrm{ref}}(t)-\dot{\phi}_{x}(t)\right), \\
& M_{y}(t)=K_{y}^{p}\left(\phi_{y}^{\mathrm{ref}}(t)-\phi_{y}(t)\right)+K_{y}^{d}\left(\dot{\phi}_{y}^{\mathrm{ref}}(t)-\dot{\phi}_{y}(t)\right),
\end{aligned}
$$

where $M_{x}$ and $M_{y}$ are the actuator moments applied at the $x$ - and $y$-hinge shown in Fig. 7.1, $K_{x}^{p}$ and $K_{y}^{p}$ are the proportional gains, $K_{x}^{d}$ and $K_{y}^{d}$ are the derivative gains, $\phi_{x}$ and $\phi_{y}$ are the current angular rotations, and $\phi_{x}^{\text {ref }}$ and $\phi_{y}^{\text {ref }}$ are the reference profiles of the controllers of the $x$ - and $y$-hinge, respectively. The implemented controller parameters, and the resulting closed-loop frequencies and damping ratios of the first two vibrational modes, are summarized in Tabs. 7.1 and 7.2. With these settings, it should be possible to obtain reasonably fast response times with a quick decay of oscillations.

\section{Reference profiles}

The IBM-u reduced model is employed for the closed-loop time-response simulation of the compliant mechanism. The objective of the controllers is to realize a motion of the

\footnotetext{
${ }^{2}$ More sophisticated controllers are at the time of writing not yet supported by the $\mathrm{C}++$ implementation of the SPACAR software.
} 
Table 7.1: Controller parameters.

\begin{tabular}{cc}
\hline$K_{x}^{p}$ & $5500[\mathrm{Nm} / \mathrm{rad}]$ \\
$K_{y}^{p}$ & $2500[\mathrm{Nm} / \mathrm{rad}]$ \\
$K_{x}^{d}$ & $40[\mathrm{Nm} \mathrm{s} / \mathrm{rad}]$ \\
$K_{y}^{d}$ & $17[\mathrm{Nm} \mathrm{s} / \mathrm{rad}]$
\end{tabular}

Table 7.2: Closed-loop frequencies and damping ratios of the first two vibrational modes.

\begin{tabular}{lcc}
\hline & Frequency $[\mathrm{Hz}]$ & Damping ratio $[-]$ \\
\hline First vibrational mode & 27.34 & 0.60 \\
Second vibrational mode & 31.74 & 0.70
\end{tabular}

end-effector along the path $\mathcal{P}(s)$ shown in Fig. 7.1. The motion must be completed within $1.5 \mathrm{~s}$ while the end-effector is stopped for $0.05 \mathrm{~s}$ at five locations along the path referred to by $s_{1}, s_{2}, s_{3}, s_{4}$ and $s_{5}$. The corresponding distances travelled along the path at these stop locations are given in Fig. 7.1.

To be able to use the controllers defined in Eq. 7.12 such that the end-effector is moving along the desired trajectory, the reference profiles $\phi_{x}^{\text {ref }}$ and $\phi_{y}^{\text {ref }}$ need to be determined as functions of time. To determine these reference profiles, a timeresponse simulation of the IBM-u reduced model is performed where all the reduced coordinates are prescribed such that the kinematics of the mechanism are completely known in advance. For this reference simulation, the reduced coordinates $\boldsymbol{\eta}$ associated with the interpolated modal subspace $\mathcal{V}(s)$ of Eq.(7.1) are prescribed to be zero, while the coordinate $s$ that defines the configuration of the mechanism along the path is prescribed as a function of time. Figure 7.4(a) shows the time-profile of the coordinate $s$. It can be seen that at the locations $s_{1}$ through $s_{5}$ the mechanism is stopped for $0.05 \mathrm{~s}$. A skew sine reference profile is used to move the end-effector from one stop to the next in $T=0.25 \mathrm{~s}$. The coordinate $s$ and its derivatives are then

$$
\begin{aligned}
& s(t)=\left(t-t_{\text {start }}\right) \frac{\Delta s}{T}-\frac{\Delta s}{2 \pi} \sin \left(\frac{2 \pi\left(t-t_{\text {start }}\right.}{T}\right), \\
& \dot{s}(t)=\frac{\Delta s}{T}\left(1-\cos \left(\frac{2 \pi\left(t-t_{\text {start }}\right.}{T}\right)\right), \\
& \ddot{s}(t)=\frac{2 \pi \Delta s}{T^{2}} \sin \left(\frac{2 \pi\left(t-t_{\text {start }}\right.}{T}\right),
\end{aligned}
$$

where $\Delta s$ is the distance between the current and the next stop location and $t_{\text {start }}$ corresponds to the time at which the mechanism starts moving from the current stop location to the next.

During a simulation of the motion of the end-effector along the path, the angular rotations of the hinge elements and the position of the end-effector are recorded. This 


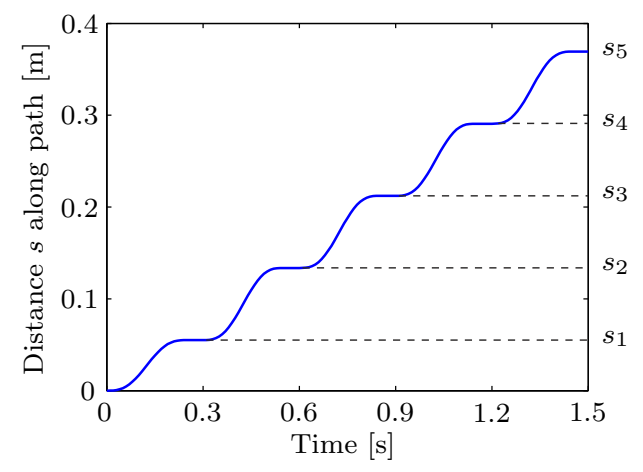

(a) Configuration coordinate $s$ prescribed as function of time

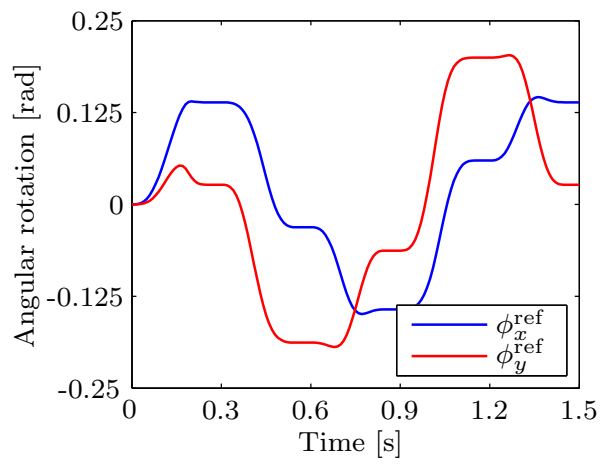

(b) Reference profiles

Figure 7.4: The configuration coordinate $s$ prescribed as a function of time (a), and the obtained reference profiles for the closed-loop time-response simulation in terms of the angular rotation angles of the $x$ - and $y$-hinge as functions of time (b).

analysis can be performed relatively quick, as the kinematics are completely known in advance. The equations of motions do not need to be time integrated for this purely kinematic analysis. Figure 7.4(b) shows the obtained angular rotations $\phi_{x}^{\text {ref }}$ and $\phi_{y}^{\text {ref }}$ as functions of time of the $x$-hinge and $y$-hinge, respectively. The recorded end-effector location $\boldsymbol{r}^{\mathrm{ref}}(t)$ as a function of time is

$$
\left\{\begin{array}{c}
x^{\mathrm{ref}}(t) \\
y^{\mathrm{ref}}(t) \\
z^{\mathrm{ref}}(t)
\end{array}\right\}=\boldsymbol{r}^{\mathrm{ref}}(t)
$$

where $x^{\text {ref }}, y^{\text {ref }}$ and $z^{\text {ref }}$ are the $x, y$ and $z$ coordinates of the end-effector. These locations are used as a reference to determine how much the end-effector is deviating from the desired path during the closed-loop time-response simulation, which is discussed next.

\section{Simulation results}

The closed-loop time-response simulation is performed by providing the reference profiles $\phi_{x}^{\text {ref }}(t)$ and $\phi_{y}^{\text {ref }}(t)$ shown in Fig. 7.4(b) to the controllers of Eq.(7.12). The effects of gravity are included by adding a gravity field $g$ of $9.81 \mathrm{~m} / \mathrm{s}^{2}$ to the model. For the time-integration of the equations of motion, the Shampine-Gordon integrator [63] is used. The model is simulated for $1.5 \mathrm{~s}$ and results are recorded for 1000 equidistant time-steps.

The coordinates $\boldsymbol{r}^{\mathrm{ee}}$ of the end-effector are recorded as a function of time and are 
given by

$$
\left\{\begin{array}{l}
x^{\mathrm{ee}}(t) \\
y^{\mathrm{e}}(t) \\
z^{\mathrm{ee}}(t)
\end{array}\right\}=\boldsymbol{r}^{\mathrm{ee}}(t)
$$

where $x^{\mathrm{ee}}, y^{\mathrm{ee}}$ and $z^{\mathrm{ee}}$ are the $x, y$ and $z$ coordinates of the end-effector. Figure 7.5(a) shows the resulting position of the end-effector $\boldsymbol{r}^{\mathrm{ee}}$ in $x$ - and $y$-direction and that of the desired path $\boldsymbol{r}^{\text {ref }}$. The error of the end-effector in $x$ - and $y$-direction is computed by

$$
\begin{aligned}
& x_{\text {error }}(t)=x^{\mathrm{ref}}(t)-x^{\mathrm{ee}}(t), \\
& y_{\text {error }}(t)=y^{\mathrm{ref}}(t)-y^{\mathrm{ee}}(t),
\end{aligned}
$$

and is shown in Fig.7.5(b). It can be observed that oscillations decay quickly when the mechanism stops at the locations $s_{1}$ through $s_{5}$ at $t=0.3 \mathrm{~s}, t=0.6 \mathrm{~s}, t=$ $0.9 \mathrm{~s}, t=1.2 \mathrm{~s}$ and $t=1.5 \mathrm{~s}$. At these locations, steady-state errors are remaining as the controllers have no integral action.

Figure 7.6 shows the $z$-displacement of the end-effector as function of time. Two simulation results are shown which differ with respect to their initial conditions. The first simulation starts from the initial undeflected configuration such that the mechanism is instantly excited in the out-of-plane direction by the gravitational forces, whereas the second simulation starts in an adjusted equilibrium configuration that accounts for gravity. The $z$-displacements obtained with the former and the latter simulation are referred to by $z_{\text {undef }}^{\mathrm{ee}}$ and $z_{\mathrm{eq}}^{\mathrm{ee}}$, respectively. This distinction is

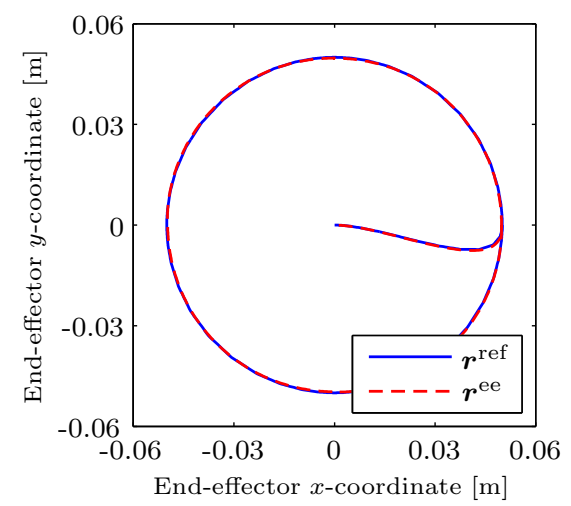

(a) End-effector trajectory

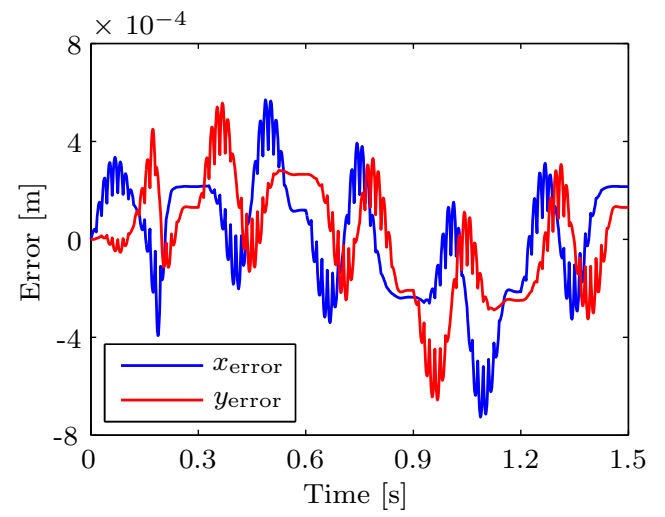

(b) End-effector error

Figure 7.5: The $x$ and $y$ coordinates of the end-effector trajectory $\boldsymbol{r}^{\text {ee }}$ and the desired path $\boldsymbol{r}^{\text {ref }}$ (a) and the errors of the end-effector location compared to the desired path in the $x$ - and $y$-directions (b). 


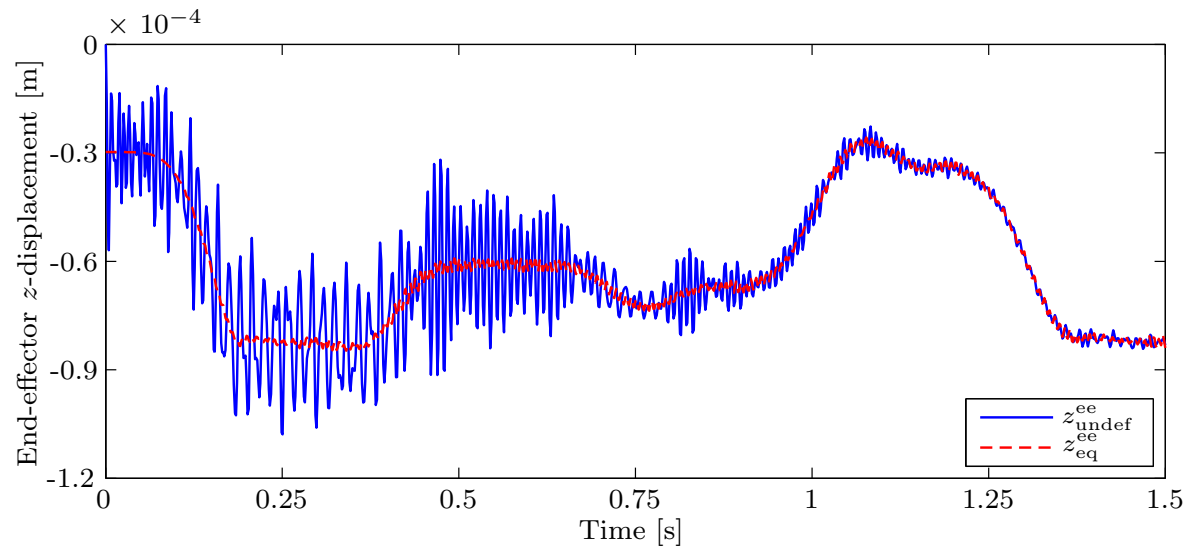

Figure 7.6: The $z$-displacement of the end-effector as function of time for the simulation starting from the initial undeflected configuration yielding $z_{\text {undef }}^{\mathrm{ee}}$, and the gravity adjusted equilibrium configuration yielding $z_{\mathrm{eq}}^{\mathrm{ee}}$.

omitted in Fig 7.5, as the effects of gravity only have minor influence on the results in the $x$ - and $y$-direction.

In the simulation that starts from the unadjusted initial configuration, the excitation by the gravitational forces causes immediate oscillations of the endeffector. The magnitude of the oscillation is slowly decaying as the corresponding vibrational modes are lightly damped by the PD controllers. It can be observed that the $z$-displacements $z_{\text {undef }}^{\mathrm{ee}}$ are correctly oscillating around the $z$-displacements $z_{\mathrm{eq}}^{\mathrm{ee}}$ of the simulation with the adjusted initial conditions. In both simulations, the end-effector initially moves downwards while the mechanism moves away from its initial configuration. During the next steps of the motion, the end-effector moves slowly upwards and downwards. As each cross-hinge in the model is deflected during this motion, its stiffness in the $z$-direction decreases with increasing deflection as is demonstrated in Chapter 3. Due to this effect, the stiffness in the $z$-direction of the complete mechanism is highly position dependent, which causes the upward and downward motion of the end-effector observed in Fig. 7.6.

Next, the frequency content of the $z_{\text {undef }}^{\mathrm{ee}}$ motion is investigated. To obtain a signal of oscillations $z_{\text {oscill }}^{\mathrm{ee}}$ with zero mean, the $z$-displacements $z_{\text {eq }}^{\mathrm{ee}}$ are subtracted from $z_{\text {undef }}^{\mathrm{ee}}$,

$$
z_{\mathrm{oscill}}^{\mathrm{ee}}(t)=z_{\mathrm{eq}}^{\mathrm{ee}}(t)-z_{\mathrm{undef}}^{\mathrm{ee}}(t) .
$$

The single-sided amplitude spectrum of $z_{\text {oscill }}^{\mathrm{ee}}$ is shown in Fig. 7.7. High peaks can be observed at approximately $65 \mathrm{~Hz}$ and $110 \mathrm{~Hz}$. By comparison with the eigenfrequency results of Fig. 7.3, it can be concluded that these peaks correspond to the third and fourth vibrational modes in the initial and the deflected configuration of the mechanism. Furthermore, it can be observed that there are no vibrational modes 


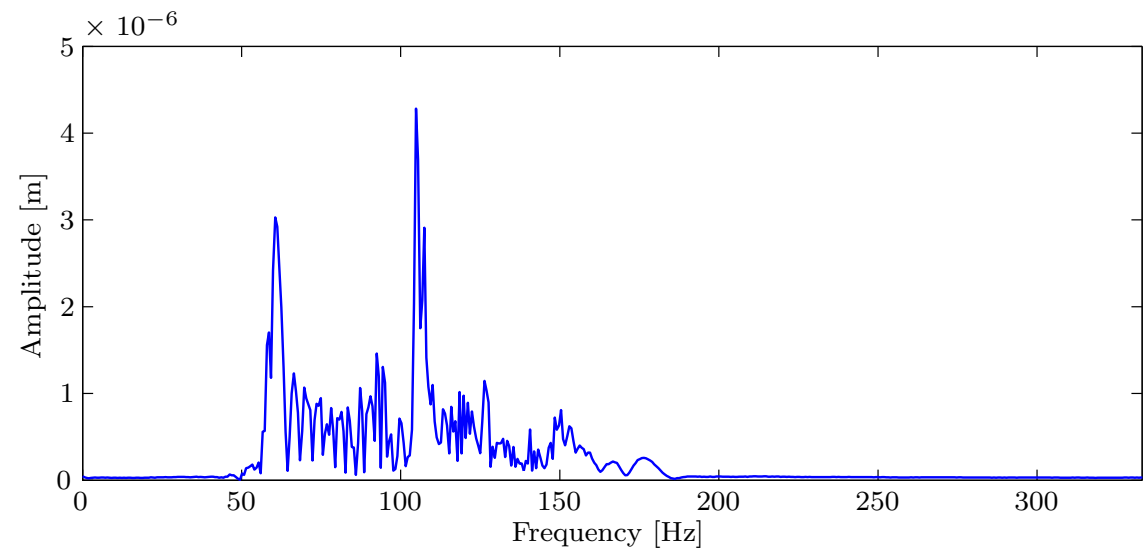

Figure 7.7: The single-sided amplitude spectrum of $z_{\mathrm{oscill}}^{\mathrm{ee}}$.

with frequencies above $180 \mathrm{~Hz}$, which can be expected as no vibrational modes with higher eigenfrequencies are present in the IBM-u reduced model as is shown in Fig. 7.3.

The simulation results presented in this section were acquired after approximately 1.5 hours of computation on a $2.20 \mathrm{GHz}$ computer. The integrator required a total of 8259 function evaluations of the model to obtain the time-response simulation results. However, for an unreduced simulation, the number of function evaluations could be in the order of millions, as is demonstrated for the compliant straight guidance example of Chapter 6 . Such a simulation would take an unreasonably long time to complete.

\subsection{Conclusions}

The IBM of Chapter 6 is further developed to be able to reduce models of mechanisms that are moving along a pre-determined path in a multi-dimensional space. Particularly, it is explained how to obtain the proper base vectors along this path for the interpolation of the modal subspace in the IBM. It is demonstrated that both constrained and unconstrained eigenvectors can be used for this purpose. Both methods are applied to reduce the SPACAR model of the two degree of freedom large-stroke compliant positioning mechanism of Chapter 4, such that a closed-loop time-response simulation can be performed.

The use of unconstrained eigenvectors shows better performance than the use of constrained eigenvectors when comparing the eigenfrequencies of the two reduced models along the path. By using the unconstrained eigenvectors, the first $n$ vibrational modes of interest are captured exactly, whereas the constrained eigenvectors were less capable of representing the higher frequency vibrational modes of interest. Furthermore, the use of the constrained eigenvectors is also less versatile as their computation requires that the coordinates that define the path are 
part of the vector of independent generalized coordinates. This is not required for the computation of the unconstrained eigenvectors. For these reasons, the use of unconstrained eigenvectors is considered superior to the use of constrained eigenvectors for the interpolation of the modal subspace.

The reduced model that uses the unconstrained eigenvectors is employed for the closed-loop time-response simulation of the mechanism where the end-effector moves along a pre-determined path. It is demonstrated that with this reduced model, results can be obtained with relatively few (approximately 8000) function evaluations. Though, due to the large number of elements in the model, each function evaluation can still take some time, adding up to a total of 1.5 hours of computation. Decreasing the computational time of each function evaluation could be achieved by further reducing the number of elements in the model. For example, each cross-hinge in the model could potentially be represented by a single element or sub-system with non-linear stiffness properties and a concentrated mass. The development and implementation of such a sub-system is far from trivial and requires further research. 


\section{Conclusions and recommendations}

\subsection{Conclusions}

The aim of this thesis is the development of model reduction techniques for flexible multibody systems to realize fast and accurate simulations of large-stroke compliant mechanisms. To that end, component and system model reduction methods are developed and applied.

In the following sections the main research objectives of Chapter 1 are restated and the conclusions of this work are summarized.

\subsubsection{Component model reduction}

Objective: The implementation of a component model reduction method for multibody system analysis of compliant mechanisms using the generalized strain approach.

In Chapter 2, a non-linear two-node superelement is derived for use in flexible multibody models based on the generalized strain approach. The superelement is shown to be capable of modelling large rigid body motions and small elastic deformations of arbitrary-shaped flexible members with two interface surfaces. The superelement is obtained by combining the Craig-Bampton substructure method with the kinematic model of a non-linear beam element, yielding a simple set of dynamic equations which can easily be implemented in flexible multibody software. This combination offers an exact representation of the superelement as a rigid body. In the derivation it is assumed that the elastic deformations remain small with respect to a co-rotational frame, allowing the mass and stiffness matrix of the superelement to be based directly on those of a reduced linear finite element (FE) model of the arbitrary-shaped flexible member. In this way, the superelement can model arbitrary-shaped flexible members provided that the assumption of small deformations is valid. 
Only the constraint modes of the Craig-Bampton method are considered in Chapter 2 for the determination of the reduced linear FE model. These modes preserve the static response to loads applied to the two nodes of the superelement. Each node is rigidly interconnected with an interface surface, allowing no local deformations of this surface. A further limitation is that the constraint modes may not be sufficient for describing the vibrational modes of the flexible member in a dynamic analysis. Therefore, in Chapter 3, the superelement is extended to support an additional set of modes. These are the fixed-interface normal modes of the Craig-Bampton method and so-called deformable-interface modes, respectively.

In the case the vibrational modes of the flexible member are inadequately represented by just the constraint modes, the fixed-interface normal modes can be added to improve the accuracy in a dynamic analysis. Furthermore, the requirement of rigid interface surfaces is relaxed as local deformations of these surfaces can be modelled with the deformable-interface modes. This allows for a compliant interconnection of superelements.

The importance of such a compliant interconnection is illustrated with an example where the large deflections of a sheet-flexure are considered. The sheetflexure has a rather large height to length ratio and as a consequence its crosssection will significantly warp when the flexure is subjected to torsional loads. To allow large deflections, multiple elements are needed to model a single sheet-flexure such that the element deformations remain small with respect to a co-rotational frame. However, if rigid interfaces are interconnecting these elements, then an artificial stiff representation of the sheet-flexure is obtained. Instead, a specific deformable-interface mode, referred to as a warping mode, is presented to model the warping of the interface surfaces. Next, a number of interconnected superelements can be used to model the sheet-flexure. It is shown that due to the inclusion of the warping modes for a compliant interconnection of the superelements, accurate dynamic results are obtained even when considering large deflections.

The practical application of superelements is further investigated in Chapter 4 where they are employed for the modelling of complex-shaped flexible members and sheet-flexures of a two degree of freedom large-stroke compliant mechanism. It is demonstrated that with the use of superelements, an accurate model of this mechanism is obtained that has significantly less degrees of freedom than a model obtained with a classical non-linear FE approach. Though, the model is still too complex to yield acceptable simulation times for time-response simulations. Therefore, further model reduction on system level is required.

\subsubsection{System model reduction}

Objective: The development of system model reduction methods applied to flexible multibody systems for fast and accurate simulations of compliant mechanisms.

In Chapters 5, 6 and 7, a system model reduction method is developed based on constraining independent generalized coordinates (i.e. the degrees of freedom) of a multibody system, such that high frequency vibrational modes are suppressed. 
Explicit-time integrators specifically benefit from the removal of the high frequency vibrational modes as larger time-steps can be taken while still providing correct results.

In Chapter 5, linear constraint relations are investigated to suppress high frequency vibrational modes of planar mechanism models. The method is referred to as the fixed basis method (FBM), where the reduction of coordinates is realized by projecting the independent generalized coordinates onto a lower dimensional subspace spanned by a set of base vectors. As the model is non-linear, only coordinates that remain small during simulation are chosen for this projection such that a minimal number of base vectors are required to describe the configuration dependency of the vibrational modes of interest. It is proposed to obtain these base vectors by performing a singular value decomposition (SVD) analysis of the vibrational modes of interest throughout the range of motion of the planar mechanism. The eigenvectors used in the SVD analysis can be weighted such that more emphasis is given to the vibrational modes with the lowest eigenfrequencies. The base vectors are chosen to correspond to the singular vectors with large corresponding singular values as these vectors describe the dominant contributions of the considered vibrational modes. It is shown that the FBM can significantly decrease the computational time of numerical simulations of planar mechanisms.

The FBM is further developed in Chapter 6 for the application to spatial compliant mechanisms. The SVD approach presented in Chapter 5 appeared to be less effective to obtain base vectors which are also capable of accurately describing more complex varying mode shapes like the out-of-plane vibrational modes and their configuration dependency. Therefore, a different SVD strategy is proposed in Chapter 6. In this approach, it is first determined which singular vectors should be retained to describe the configuration dependency of each vibrational mode separately using SVD analyses. Subsequently, the singular vectors obtained from the SVD analyses are grouped and subjected to an overall SVD analysis to remove any linear dependency in order to obtain a minimal number of base vectors. It is shown that with this method, the vibrational modes of interest can be accurately described over the full range of motion. However, as multiple base vectors are still required to describe the configuration dependency of each of the vibrational modes of interest, the inclusion of unwanted high frequency vibrational modes in the reduced model is unavoidable.

Therefore, in Chapters 6, another method is developed that uses non-linear constraint relations, yielding a so-called interpolated basis method (IBM). Here, the independent generalized coordinates are described throughout the range of motion by an interpolation of reference configurations and eigenvectors that represent the vibrational modes of interest. The interpolation of these eigenvectors makes it possible to take into account the configuration dependency of the vibrational modes without having any unwanted high frequency modes in the reduced model. However, a direct interpolation can lead to large derivatives of the interpolated eigenvectors due to mode-crossing and mode-veering phenomena. This in turn causes large time derivatives that deteriorate the accuracy of the integration of the reduced equations of motion in a numerical simulation. Therefore, a so-called modal subspace interpolation (MSI) algorithm is proposed where base vectors are 
determined that span the same modal subspace as the eigenvectors of interest, but vary as little as possible with a change in the configuration. This is accomplished by a successive projection of the base vectors onto the modal subspace of the next configuration throughout the trajectory of motion. It is shown that this approach allows for an accurate interpolation of the modal subspace without having to resort to mode-tracking algorithms to deal with the mode-crossing and mode-veering phenomena. Furthermore, it is demonstrated that an IBM reduced model is numerically more efficient than an FBM reduced model.

Finally, in Chapter 7, some improvements are made to the IBM such that it can successfully be applied for the reduction of the two degree of freedom large-stroke compliant mechanism model developed in Chapter 4. It is shown that with the IBM an impressive over-all-reduction of the model complexity can be obtained, while maintaining accuracy along a pre-determined path of the end-effector of the compliant mechanism. It is demonstrated that with this reduced model of the compliant mechanism, a closed-loop time-response simulation can be performed with reasonably fast simulation times.

\subsection{Recommendations}

In this thesis, reduction methods are developed for the efficient and accurate simulation of compliant mechanisms. The applicability and performance of these methods can be further improved by research on the following topics:

- The development of superelements that are not restricted to a two-noded formulation. This could significantly extend the modelling capabilities of the proposed superelement approach. The main challenge in the development of $n$-node superelements is to find a suitable description for the deformation coordinates expressed in terms of the absolute nodal coordinates. Note that for the inclusion in the generalized strain approach, the first and second order derivatives of the deformation coordinates also need to be determined.

- The investigation of non-linear sub-system reduction methods, where a single sub-system represents an assembly of non-linear elements. This type of reduction combines aspects of both component and system model reduction. A multibody model can then be modelled with an assembly of sub-systems and traditional elements. This is for instance useful for the efficient modelling of the two degree of freedom compliant mechanisms where the cross-hinge models could be replaced by computationally less expensive non-linear sub-systems.

- Investigating the feasibility of an IBM with more than a single configuration coordinate. This would allow the development of non-linear reduced models that are not restricted to a pre-determined path. Though, due to the curse of dimensionality, the number of required reference configurations inhibits a straightforward implementation of such an approach.

During the realization of this work, a significant effort has been delivered in the design and realization of the two degree of freedom large-stroke compliant mechanism. 
The design of this mechanism was a main motivation for the development of the model reduction methods presented in this thesis. Further improvements on the design of this mechanism can be made by:

- Investigating the differences between the measurement and model results as they are observed in [27, 70]. This could lead to an improved understanding of the characteristics of compliant mechanisms and to the development of more reliable models. Furthermore, design and manufacturing guidelines could be determined such that the behaviour of the compliant mechanism is in closer agreement with the developed models.

- Improving the performance of the large-stroke compliant mechanism by redesigning it with different types of cross-hinges such as the promising infinity flexure [69]. The developed model reduction methods can be used for a relatively quick evaluation of the performance. 

Appendices 



\section{Transformation of velocities}

In this appendix, the absolute velocities of the nodes of the FE model of a superelement, are rewritten in terms of the floating frame of reference.

For infinitesimal elastic deformations, the absolute velocities of the $i^{\text {th }}$ node of the FE model, $\dot{\overline{\boldsymbol{u}}}^{(i)}$, expressed in the reference frame attached to superelement node $p$, can be written as

$$
\begin{aligned}
\dot{\overline{\boldsymbol{u}}}^{(i)}= & {\left[\boldsymbol{V}_{1}^{(i)}, \boldsymbol{V}_{2}^{(i)}, \boldsymbol{V}_{3}^{(i)}\right] \boldsymbol{R}_{0}^{\mathrm{T}} \boldsymbol{R}^{p \mathrm{~T}} \boldsymbol{v}^{p}+\left[\boldsymbol{V}_{4}^{(i)}, \boldsymbol{V}_{5}^{(i)}, \boldsymbol{V}_{6}^{(i)}\right] \boldsymbol{R}_{0}^{\mathrm{T}} \boldsymbol{R}^{p \mathrm{~T}} \boldsymbol{\omega}^{p} } \\
+ & {\left[\boldsymbol{V}_{7}^{(i)}, \boldsymbol{V}_{8}^{(i)}, \boldsymbol{V}_{9}^{(i)}\right] \boldsymbol{R}_{0}^{\mathrm{T}} \boldsymbol{R}^{p \mathrm{~T}} \boldsymbol{v}^{q}+\left[\boldsymbol{V}_{10}^{(i)}, \boldsymbol{V}_{11}^{(i)}, \boldsymbol{V}_{12}^{(i)}\right] \boldsymbol{R}_{0}^{\mathrm{T}} \boldsymbol{R}^{p \mathrm{~T}} \boldsymbol{\omega}^{q} }
\end{aligned}
$$

where $\boldsymbol{V}_{n}^{(i)}$ is the vector with the $x, y$ and $z$ contributions of the $n^{\text {th }}$ constraint mode for the $i^{\text {th }}$ node of the FE model.

Rigid body translations and infinitesimal rigid body rotations can be expressed as linear combinations of the constraint modes,

$$
\begin{aligned}
& \boldsymbol{I}=\left[\boldsymbol{V}_{1}^{(i)}, \boldsymbol{V}_{2}^{(i)}, \boldsymbol{V}_{3}^{(i)}\right]+\left[\boldsymbol{V}_{7}^{(i)}, \boldsymbol{V}_{8}^{(i)}, \boldsymbol{V}_{9}^{(i)}\right], \\
&\left\{\begin{array}{l}
1 \\
0 \\
0
\end{array}\right\} \times \overline{\boldsymbol{r}}_{0}^{(i)}=\boldsymbol{V}_{4}^{(i)}+\boldsymbol{V}_{10}^{(i)}, \\
&\left\{\begin{array}{l}
0 \\
1 \\
0
\end{array}\right\} \times \overline{\boldsymbol{r}}_{0}^{(i)}=\boldsymbol{V}_{5}^{(i)}+\boldsymbol{V}_{11}^{(i)}-l_{0} \boldsymbol{V}_{9}^{(i)}, \\
&\left\{\begin{array}{l}
0 \\
0 \\
1
\end{array}\right\} \times \overline{\boldsymbol{r}}_{0}^{(i)}=\boldsymbol{V}_{6}^{(i)}+\boldsymbol{V}_{12}^{(i)}+l_{0} \boldsymbol{V}_{8}^{(i)},
\end{aligned}
$$

where $\overline{\boldsymbol{r}}_{0}$ is the position vector of the nodes in the FE model in undeformed configuration. Substituting in equation (A.1) for $\boldsymbol{V}_{1}$ through $\boldsymbol{V}_{6}$ the expressions of 
equation (A.2) gives

$$
\dot{\overline{\boldsymbol{u}}}^{(i)}=\boldsymbol{R}_{0}^{\mathrm{T}} \boldsymbol{R}^{p \mathrm{~T}} \boldsymbol{v}^{p}+\left(\boldsymbol{R}_{0}^{\mathrm{T}} \boldsymbol{R}^{p \mathrm{~T}} \boldsymbol{\omega}^{p}\right) \times \overline{\boldsymbol{r}}_{0}^{(i)}+\dot{\boldsymbol{e}}^{(i)},
$$

with

$$
\dot{\overline{\boldsymbol{e}}}^{(i)}=\left[\boldsymbol{V}_{7}^{(i)}, \boldsymbol{V}_{8}^{(i)}, \boldsymbol{V}_{9}^{(i)}, \boldsymbol{V}_{10}^{(i)}, \boldsymbol{V}_{11}^{(i)}, \boldsymbol{V}_{12}^{(i)}\right]\left\{\begin{array}{c}
\bar{v}_{x}^{q}-\bar{v}_{x}^{p} \\
\bar{v}_{y}^{q}-\bar{v}_{y}^{p}-l_{0} \bar{\omega}_{z}^{p} \\
\bar{v}_{z}^{q}-\bar{v}_{z}^{p}+l_{0} \bar{\omega}_{y}^{p} \\
\bar{\omega}_{x}^{q}-\bar{\omega}_{x}^{p} \\
\bar{\omega}_{y}^{q}-\bar{\omega}_{y}^{p} \\
\bar{\omega}_{z}^{q}-\bar{\omega}_{z}^{p}
\end{array}\right\}
$$

and

$$
\begin{gathered}
\left\{\begin{array}{c}
\bar{v}_{x}^{p} \\
\bar{v}_{y}^{p} \\
\bar{v}_{z}^{p}
\end{array}\right\}=\boldsymbol{R}_{0}^{\mathrm{T}} \boldsymbol{R}^{p \mathrm{~T}} \boldsymbol{v}^{p}, \quad\left\{\begin{array}{c}
\bar{v}_{x}^{q} \\
\bar{v}_{y}^{q} \\
\bar{v}_{z}^{q}
\end{array}\right\}=\boldsymbol{R}_{0}^{\mathrm{T}} \boldsymbol{R}^{p \mathrm{~T}} \boldsymbol{v}^{q}, \\
\left\{\begin{array}{c}
\bar{\omega}_{x}^{p} \\
\bar{\omega}_{y}^{p} \\
\bar{\omega}_{z}^{p}
\end{array}\right\}=\boldsymbol{R}_{0}^{\mathrm{T}} \boldsymbol{R}^{p \mathrm{~T}} \boldsymbol{\omega}^{p}, \quad\left\{\begin{array}{c}
\bar{\omega}_{x}^{q} \\
\bar{\omega}_{y}^{q} \\
\bar{\omega}_{z}^{q}
\end{array}\right\}=\boldsymbol{R}_{0}^{\mathrm{T}} \boldsymbol{R}^{p \mathrm{~T}} \boldsymbol{\omega}^{q} .
\end{gathered}
$$




\section{Expressions for $\dot{B}$}

In this appendix the time derivatives of the velocity transformation matrices $\boldsymbol{B}_{1}, \boldsymbol{B}_{2}$ and $\boldsymbol{B}_{3}$ are given (see equation 2.38).

Time derivative of $\boldsymbol{B}_{1}$

Taking the time derivative of matrix $\boldsymbol{B}_{1}$ in equation (2.24) gives

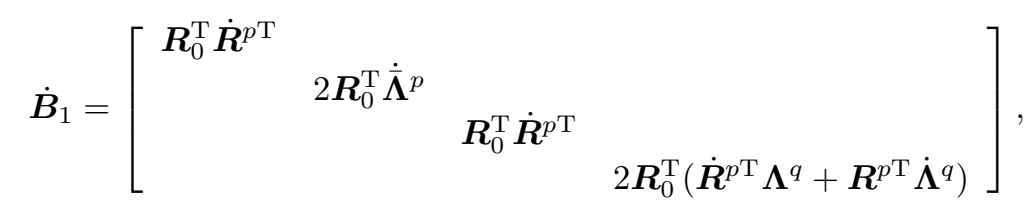

where the time derivative of $\boldsymbol{R}^{p}$ can be computed by

$$
\dot{\boldsymbol{R}}^{p}=2 \dot{\boldsymbol{\Lambda}}^{p} \overline{\boldsymbol{\Lambda}}^{p \mathrm{~T}}
$$

where $\overline{\boldsymbol{\Lambda}}^{p}$ is defined in equation 2.33 .

Time derivative of $\boldsymbol{B}_{2}$

Taking the time derivative of matrix $\boldsymbol{B}_{2}$ in equation (2.31) gives

$$
\dot{\boldsymbol{B}}_{2}=\left[\begin{array}{ccc}
\boldsymbol{R}_{0}^{\mathrm{T}} \dot{\boldsymbol{R}}^{r \mathrm{~T}} & & \\
& 2 \boldsymbol{R}_{0}^{\mathrm{T}}\left(\dot{\boldsymbol{R}}^{r \mathrm{~T}} \boldsymbol{\Lambda}^{p}+\boldsymbol{R}^{r \mathrm{~T}} \dot{\boldsymbol{\Lambda}}^{p}\right) & \\
& \boldsymbol{R}_{0}^{\mathrm{T}} \dot{\boldsymbol{R}}^{r \mathrm{~T}} & \\
& & 2 \boldsymbol{R}_{0}^{\mathrm{T}}\left(\dot{\boldsymbol{R}}^{r \mathrm{~T}} \boldsymbol{\Lambda}^{q}+\boldsymbol{R}^{r \mathrm{~T}} \dot{\boldsymbol{\Lambda}}^{q}\right)
\end{array}\right]
$$

where the time derivative of $\boldsymbol{R}^{r}$ is

$$
\dot{\boldsymbol{R}}^{r}=2 \dot{\boldsymbol{\Lambda}}^{r} \overline{\boldsymbol{\Lambda}}^{r \mathrm{~T}}
$$


Here the evaluation of equation (B.4) requires an expression for $\dot{\boldsymbol{\lambda}}^{r}$. Taking the derivative of $\boldsymbol{\lambda}^{r}$ in equation (2.29) gives

$$
\dot{\boldsymbol{\lambda}}^{r}=\frac{\partial \boldsymbol{\lambda}^{r}}{\partial \boldsymbol{\lambda}^{p}} \dot{\boldsymbol{\lambda}}^{p}+\frac{\partial \boldsymbol{\lambda}^{r}}{\partial \boldsymbol{\lambda}^{q}} \dot{\boldsymbol{\lambda}}^{q} .
$$

It can be shown that

$$
\frac{\partial \boldsymbol{\lambda}^{r}}{\partial \boldsymbol{\lambda}^{p}}=\frac{\partial \boldsymbol{\lambda}^{r}}{\partial \boldsymbol{\lambda}^{q}}=\frac{\boldsymbol{I}-\boldsymbol{\lambda}^{r} \boldsymbol{\lambda}^{r \mathrm{~T}}}{\left\|\boldsymbol{\lambda}^{p}+\boldsymbol{\lambda}^{q}\right\|}=\frac{\boldsymbol{\Lambda}^{r \mathrm{~T}} \boldsymbol{\Lambda}^{r}}{\left\|\boldsymbol{\lambda}^{p}+\boldsymbol{\lambda}^{q}\right\|} .
$$

Substituting equation (B.6) in equation (B.5) then gives

$$
\dot{\boldsymbol{\lambda}}^{r}=\frac{\boldsymbol{\Lambda}^{r \mathrm{~T}} \boldsymbol{\Lambda}^{r}}{\left\|\boldsymbol{\lambda}^{p}+\boldsymbol{\lambda}^{q}\right\|}\left(\dot{\boldsymbol{\lambda}}^{p}+\dot{\boldsymbol{\lambda}}^{q}\right) .
$$

Time derivative of $B_{3}$

Taking the time derivative of matrix $\boldsymbol{B}_{3}$ in equation (2.32) gives

$$
\dot{\boldsymbol{B}}_{3}=\left[\begin{array}{cccc}
\boldsymbol{R}_{0}^{\mathrm{T}} \dot{\boldsymbol{R}}^{r \mathrm{~T}} & & & \\
& 2 \boldsymbol{R}_{0}^{\mathrm{T}} \dot{\overline{\boldsymbol{\Lambda}}}^{p} & & \\
& & \boldsymbol{R}_{0}^{\mathrm{T}} \dot{\boldsymbol{R}}^{r \mathrm{~T}} & \\
& & & 2 \boldsymbol{R}_{0}^{\mathrm{T}} \dot{\overline{\boldsymbol{\Lambda}}}^{q}
\end{array}\right] .
$$




\section{Expressions for $B^{*}$}

In this appendix expressions are given for $\boldsymbol{B}_{1}^{*}, \boldsymbol{B}_{2}^{*}$ and $\boldsymbol{B}_{3}^{*}$ (see equation 2.38).

Expression for $B_{1}^{*}$

For $\boldsymbol{B}_{1}^{*}$ we can write

$$
\boldsymbol{B}_{1}^{*}=\frac{\partial \overline{\boldsymbol{v}}}{\partial \boldsymbol{x}}=\left[\begin{array}{cccc}
\mathbf{0} & \frac{\partial \overline{\boldsymbol{v}}^{p}}{\partial \boldsymbol{\lambda}^{p}} & \mathbf{0} & \mathbf{0} \\
\mathbf{0} & \frac{\partial \overline{\boldsymbol{\omega}}^{p}}{\partial \boldsymbol{\lambda}^{p}} & \mathbf{0} & \mathbf{0} \\
\mathbf{0} & \frac{\partial \overline{\boldsymbol{v}}^{q}}{\partial \boldsymbol{\lambda}^{p}} & \mathbf{0} & \mathbf{0} \\
\mathbf{0} & \frac{\partial \overline{\boldsymbol{\omega}}^{q}}{\partial \boldsymbol{\lambda}^{p}} & \mathbf{0} & \frac{\partial \overline{\boldsymbol{\omega}}^{q}}{\partial \boldsymbol{\lambda}^{q}}
\end{array}\right]
$$

where

$$
\begin{aligned}
\frac{\partial \bar{v}_{i}^{p}}{\partial \lambda_{j}^{p}} & =R_{0 k i} \frac{\partial R_{l k}^{p}}{\partial \lambda_{j}^{p}} \dot{r}_{l}^{p}, \\
\frac{\partial \bar{\omega}_{i}^{p}}{\partial \lambda_{j}^{p}} & =-2 R_{0 k i} \dot{\bar{\Lambda}}_{k j}^{p}, \\
\frac{\partial \bar{v}_{i}^{q}}{\partial \lambda_{j}^{p}} & =R_{0 k i} \frac{\partial R_{l k}^{p}}{\partial \lambda_{j}^{p}} \dot{r}_{l}^{q}, \\
\frac{\partial \bar{\omega}_{i}^{q}}{\partial \lambda_{j}^{p}} & =2 R_{0 k i} \frac{\partial R_{l k}^{p}}{\partial \lambda_{j}^{p}} \Lambda_{l m}^{q} \dot{\lambda}_{m}^{q}, \\
\frac{\partial \bar{\omega}_{i}^{q}}{\partial \lambda_{j}^{q}} & =-2 R_{0 k i} R_{l k}^{p} \dot{\Lambda}_{l j}^{q} .
\end{aligned}
$$


The derivative of a rotation matrix to its Euler parameters is,

$$
\begin{aligned}
& \frac{\partial \boldsymbol{R}}{\partial \lambda_{1}}=2\left[\begin{array}{rrr}
\lambda_{0} & -\lambda_{3} & \lambda_{2} \\
\lambda_{3} & \lambda_{0} & -\lambda_{1} \\
-\lambda_{2} & \lambda_{1} & \lambda_{0}
\end{array}\right], \\
& \frac{\partial \boldsymbol{R}}{\partial \lambda_{2}}=2\left[\begin{array}{rrr}
\lambda_{1} & \lambda_{2} & \lambda_{3} \\
\lambda_{2} & -\lambda_{1} & -\lambda_{0} \\
\lambda_{3} & \lambda_{0} & -\lambda_{1}
\end{array}\right], \\
& \frac{\partial \boldsymbol{R}}{\partial \lambda_{3}}=2\left[\begin{array}{rrr}
-\lambda_{2} & \lambda_{1} & \lambda_{0} \\
\lambda_{1} & \lambda_{2} & \lambda_{3} \\
-\lambda_{0} & \lambda_{3} & -\lambda_{2}
\end{array}\right], \\
& \frac{\partial \boldsymbol{R}}{\partial \lambda_{4}}=2\left[\begin{array}{rrr}
-\lambda_{3} & -\lambda_{0} & \lambda_{1} \\
\lambda_{0} & -\lambda_{3} & \lambda_{2} \\
\lambda_{1} & \lambda_{2} & \lambda_{3}
\end{array}\right],
\end{aligned}
$$

which can be used to evaluate $\frac{\partial \boldsymbol{R}^{p}}{\partial \boldsymbol{\lambda}^{p}}$ in equation (C.2).

\section{Expression for $B_{2}^{*}$}

For $\boldsymbol{B}_{2}^{*}$ we can write

$$
\boldsymbol{B}_{2}^{*}=\frac{\partial \overline{\boldsymbol{v}}}{\partial \boldsymbol{x}}=\left[\begin{array}{cccc}
\mathbf{0} & \frac{\partial \overline{\boldsymbol{v}}^{p}}{\partial \boldsymbol{\lambda}^{p}} & \mathbf{0} & \frac{\partial \overline{\boldsymbol{v}}^{p}}{\partial \boldsymbol{\lambda}^{q}} \\
\mathbf{0} & \frac{\partial \overline{\boldsymbol{\omega}}^{p}}{\partial \boldsymbol{\lambda}^{p}} & \mathbf{0} & \frac{\partial \overline{\boldsymbol{\omega}}^{p}}{\partial \boldsymbol{\lambda}^{q}} \\
\mathbf{0} & \frac{\partial \overline{\boldsymbol{v}}^{q}}{\partial \boldsymbol{\lambda}^{p}} & \mathbf{0} & \frac{\partial \overline{\boldsymbol{v}}^{q}}{\partial \boldsymbol{\lambda}^{q}} \\
\mathbf{0} & \frac{\partial \overline{\boldsymbol{\omega}}^{q}}{\partial \boldsymbol{\lambda}^{p}} & \mathbf{0} & \frac{\partial \overline{\boldsymbol{\omega}}^{q}}{\partial \boldsymbol{\lambda}^{q}}
\end{array}\right]
$$


where

$$
\begin{aligned}
\frac{\partial \bar{v}_{i}^{p}}{\partial \lambda_{j}^{p}} & =R_{0 k i} \frac{\partial R_{l k}^{r}}{\partial \lambda_{j}^{p}} \dot{r}_{l}^{p}, \\
\frac{\partial \bar{v}_{i}^{p}}{\partial \lambda_{j}^{q}} & =\frac{\partial \bar{v}_{i}^{p}}{\partial \lambda_{j}^{p}}, \\
\frac{\partial \bar{\omega}_{i}^{p}}{\partial \lambda_{j}^{p}} & =\frac{\partial \bar{\omega}_{i}^{p}}{\partial \lambda_{j}^{q}}-2 R_{0 k i} R_{l k}^{r} \dot{\Lambda}_{l j}^{p}, \\
\frac{\partial \bar{\omega}_{i}^{p}}{\partial \lambda_{j}^{q}} & =2 R_{0 k i} \frac{\partial R_{l k}^{r}}{\partial \lambda_{j}^{p}} \Lambda_{l m}^{p} \dot{\lambda}_{m}^{p}, \\
\frac{\partial \bar{v}_{i}^{q}}{\partial \lambda_{j}^{p}} & =R_{0 k i} \frac{\partial R_{l k}^{r}}{\partial \lambda_{j}^{p}} \dot{r}_{l}^{q}, \\
\frac{\partial \bar{v}_{i}^{q}}{\partial \lambda_{j}^{q}} & =\frac{\partial \bar{v}_{i}^{q}}{\partial \lambda_{j}^{p}}, \\
\frac{\partial \bar{\omega}_{i}^{q}}{\partial \lambda_{j}^{p}} & =2 R_{0 k i} \frac{\partial R_{l k}^{r}}{\partial \lambda_{j}^{p}} \Lambda_{l m}^{q} \dot{\lambda}_{m}^{q}, \\
\frac{\partial \bar{\omega}_{i}^{q}}{\partial \lambda_{j}^{q}} & =\frac{\partial \bar{\omega}_{i}^{q}}{\partial \lambda_{j}^{p}}-2 R_{0 k i} R_{l k}^{r} \dot{\Lambda}_{l j}^{q} .
\end{aligned}
$$

The tensor $\frac{\partial R_{l k}^{r}}{\partial \lambda_{j}^{p}}$ can be expressed as

$$
\frac{\partial R_{l k}^{r}}{\partial \lambda_{j}^{p}}=\frac{\partial R_{l k}^{r}}{\partial \lambda_{n}^{r}} \frac{\partial \lambda_{n}^{r}}{\partial \lambda_{j}^{p}},
$$

and can be evaluated using equation (B.6) and equation (C.3).

\section{Expression for $B_{3}^{*}$}

For $\boldsymbol{B}_{3}^{*}$ we can write

$$
\boldsymbol{B}_{3}^{*}=\frac{\partial \overline{\boldsymbol{v}}}{\partial \boldsymbol{x}}=\left[\begin{array}{cccc}
\mathbf{0} & \frac{\partial \overline{\boldsymbol{v}}^{p}}{\partial \boldsymbol{\lambda}^{p}} & \mathbf{0} & \frac{\partial \overline{\boldsymbol{v}}^{p}}{\partial \boldsymbol{\lambda}^{q}} \\
\mathbf{0} & \frac{\partial \overline{\boldsymbol{\omega}}^{p}}{\partial \boldsymbol{\lambda}^{p}} & \mathbf{0} & \mathbf{0} \\
\mathbf{0} & \frac{\partial \overline{\boldsymbol{v}}^{q}}{\partial \boldsymbol{\lambda}^{p}} & \mathbf{0} & \frac{\partial \overline{\boldsymbol{v}}^{q}}{\partial \boldsymbol{\lambda}^{q}} \\
\mathbf{0} & \mathbf{0} & \mathbf{0} & \frac{\partial \overline{\boldsymbol{\omega}}^{q}}{\partial \boldsymbol{\lambda}^{q}}
\end{array}\right]
$$


where $\frac{\partial \overline{\boldsymbol{v}}^{p}}{\partial \boldsymbol{\lambda}^{p}}, \frac{\partial \overline{\boldsymbol{v}}^{p}}{\partial \boldsymbol{\lambda}^{q}}, \frac{\partial \overline{\boldsymbol{v}}^{q}}{\partial \overline{\boldsymbol{\lambda}}^{p}}$ and $\frac{\partial \overline{\boldsymbol{v}}^{q}}{\partial \boldsymbol{\lambda}^{q}}$ are the same expressions as given by equation (C.5). Furthermore, $\frac{\partial \overline{\boldsymbol{\omega}}^{p}}{\partial \boldsymbol{\lambda}^{p}}$ and $\frac{\partial \overline{\boldsymbol{\omega}}^{q}}{\partial \boldsymbol{\lambda}^{q}}$ are given by

$$
\frac{\partial \overline{\boldsymbol{\omega}}^{p}}{\partial \boldsymbol{\lambda}^{p}}=-2 \boldsymbol{R}_{0}^{\mathrm{T}} \dot{\bar{\Lambda}}^{p} \quad \text { and } \quad \frac{\partial \overline{\boldsymbol{\omega}}^{q}}{\partial \boldsymbol{\lambda}^{q}}=-2 \boldsymbol{R}_{0}^{\mathrm{T}} \dot{\boldsymbol{\Lambda}}^{q} .
$$




\section{Equations of motion of a multibody system containing superelements}

In this appendix, the equations of motion as a set of non-linear second order ordinary differential equations are derived for a system which contains superelements. These are the equations that are solved in Chapters 3 and 4 to obtain the simulation results.

Following the approach described in [2], a mechanism can be built up with finite elements, such as superelements, beam or hinge elements, by letting them have nodal coordinates in common. Unlike these other types of elements, the superelements can also share the generalized coordinates of Eq.(3.15). The assemblage of elements is realized by defining a vector $\boldsymbol{x}_{n}$ of nodal coordinates and a vector $\overline{\boldsymbol{\eta}}$, that contains generalized coordinates of the superelements, for the entire system. The deformation functions of the elements of Eq.(3.14a), can be assembled to express the deformation coordinates $\varepsilon_{d}$ of all elements in terms of the vector $\boldsymbol{x}_{n}$,

$$
\varepsilon_{d}=\mathcal{D}\left(\boldsymbol{x}_{n}\right)
$$

For convenience, Eq.(D.1) is appended with the trivial relation $\overline{\boldsymbol{\eta}}=\overline{\boldsymbol{\eta}}$ yielding

$$
\left\{\begin{array}{c}
\boldsymbol{\varepsilon}_{d} \\
\overline{\boldsymbol{\eta}}
\end{array}\right\}=\left\{\begin{array}{c}
\mathcal{D}\left(\boldsymbol{x}_{n}\right) \\
\overline{\boldsymbol{\eta}}
\end{array}\right\} .
$$

Next, the equations of motion are derived in terms of a minimal set of independent generalized coordinates $\boldsymbol{q}$. This vector can consists of a subset of nodal coordinates $\boldsymbol{x}_{n}^{(m)}$ and deformation coordinates $\boldsymbol{\varepsilon}_{d}^{(m)}$. The generalized coordinates $\overline{\boldsymbol{\eta}}$ are by definition independent and should be included in the vector $\boldsymbol{q}$, yielding

$$
\boldsymbol{q}=\left\{\begin{array}{c}
\boldsymbol{x}_{n}^{(m)} \\
\boldsymbol{\varepsilon}_{d}^{(m)} \\
\overline{\boldsymbol{\eta}}
\end{array}\right\}
$$


The vector $\boldsymbol{q}$ represents the minimal number of coordinates required to describe, together with any kinematic constraints, the configuration of the mechanism. Solving Eq.(D.2) for the vector $\boldsymbol{q}$ then yields the geometric transfer functions $\mathcal{F}$ and $\mathcal{E}$ that define the mechanism configuration,

$$
\begin{gathered}
\boldsymbol{x}=\mathcal{F}(\boldsymbol{q}), \quad \boldsymbol{x}=\left\{\begin{array}{c}
\boldsymbol{x}_{n} \\
\overline{\boldsymbol{\eta}}
\end{array}\right\}, \\
\boldsymbol{\varepsilon}=\mathcal{E}(\boldsymbol{q}), \quad \boldsymbol{\varepsilon}=\left\{\begin{array}{c}
\boldsymbol{\varepsilon}_{d} \\
\overline{\boldsymbol{\eta}}
\end{array}\right\} .
\end{gathered}
$$

Note that these equations still contain the trivial relation $\overline{\boldsymbol{\eta}}=\overline{\boldsymbol{\eta}}$, whereas the vectors $\boldsymbol{x}_{n}$ and $\varepsilon_{d}$ are computed numerically in an iterative way [38]. Here, the vectors $\boldsymbol{x}$ and $\boldsymbol{\varepsilon}$ are introduced to be in line with their counterparts in Chapters 5 and 6. Taking the first and second time derivative of Eq.(D.4a) yields the system velocities and accelerations:

$$
\begin{gathered}
\dot{\boldsymbol{x}}=\mathcal{F}_{, q} \dot{\boldsymbol{q}}, \\
\ddot{\boldsymbol{x}}=\left(\mathcal{F}_{, q q} \dot{\boldsymbol{q}}\right) \dot{\boldsymbol{q}}+\mathcal{F}_{, q} \ddot{\boldsymbol{q}},
\end{gathered}
$$

Let $\boldsymbol{M}_{\boldsymbol{x}}(\boldsymbol{x})$ be the global mass matrix, obtained by assembling the mass matrices, and let $\boldsymbol{f}(\boldsymbol{x}, \dot{\boldsymbol{x}}, t)$ be the vector of external nodal forces, including gravitational forces and the velocity dependent inertia forces. Furthermore, let the loading state of each element be described by a vector of stress resultants, collected in the assembled vector $\boldsymbol{\sigma}$ which is dual to the vector $\varepsilon$ of Eq.(D.4b). Then, according to the principle of virtual work we obtain for the equations of motion of the mechanism

$$
\delta \boldsymbol{x}^{\mathrm{T}}\left(\boldsymbol{f}-\boldsymbol{M}_{\boldsymbol{x}} \ddot{\boldsymbol{x}}\right)=\delta \boldsymbol{\varepsilon}^{\mathrm{T}} \boldsymbol{\sigma}
$$

for all virtual variations $\delta \boldsymbol{x}$ and $\delta \varepsilon$ which satisfy the instantaneous kinematic constraints

$$
\delta \boldsymbol{x}=\mathcal{F}_{, \boldsymbol{q}} \delta \boldsymbol{q} \quad \text { and } \quad \delta \varepsilon=\mathcal{E}_{, \boldsymbol{q}} \delta \boldsymbol{q} .
$$

Substituting Eqs. (D.5) and (D.7) in Eq. (D.6) gives

$$
\delta \boldsymbol{q}^{\mathrm{T}} \boldsymbol{M}_{\boldsymbol{q}} \ddot{\boldsymbol{q}}=\delta \boldsymbol{q}^{\mathrm{T}}\left(\mathcal{F}_{, \boldsymbol{q}}^{\mathrm{T}}\left(\boldsymbol{f}-\boldsymbol{M}_{\boldsymbol{x}}\left(\mathcal{F}_{, \boldsymbol{q} \boldsymbol{q}} \dot{\boldsymbol{q}}\right) \dot{\boldsymbol{q}}\right)-\mathcal{E}_{, \boldsymbol{q}}^{\mathrm{T}} \boldsymbol{\sigma}\right)
$$

with

$$
M_{\boldsymbol{q}}=\mathcal{F}_{, \boldsymbol{q}}^{\mathrm{T}} M_{\boldsymbol{x}} \mathcal{F}_{, \boldsymbol{q}}
$$

Since the components of the vector $\delta \boldsymbol{q}$ are independent, we obtain the equations of motion

$$
M_{\boldsymbol{q}} \ddot{\boldsymbol{q}}=\mathcal{F}_{, \boldsymbol{q}}^{\mathrm{T}}\left(\boldsymbol{f}-\boldsymbol{M}_{\boldsymbol{x}}\left(\mathcal{F}_{, \boldsymbol{q} q} \dot{\boldsymbol{q}}\right) \dot{\boldsymbol{q}}\right)-\mathcal{E}_{, \boldsymbol{q}}^{\mathrm{T}} \boldsymbol{\sigma}
$$

expressed as a set of non-linear second order ordinary differential equations. 


\section{Linearized equations of motion}

In this appendix the linearized equations of motion are derived as they appear in [40]. The mass and stiffness matrices derived here are used to compute the eigenfrequency results in Chapters 3, 4, 5, 6 and $\%$.

Substituting Eq. (D.7) in Eq. (D.6) and dropping the virtual variations $\delta \boldsymbol{q}$ yields the vector function $\boldsymbol{g}(\boldsymbol{q}, \dot{\boldsymbol{q}}, \ddot{\boldsymbol{q}})$

$$
\boldsymbol{g}=\mathcal{F}_{, \boldsymbol{q}}^{\mathrm{T}}\left(\boldsymbol{M}_{\boldsymbol{x}} \ddot{\boldsymbol{x}}-\boldsymbol{f}\right)+\mathcal{E}_{, \boldsymbol{q}}^{\mathrm{T}} \boldsymbol{\sigma}=\mathbf{0}
$$

which is an equivalent representation of the equations of motion (D.10). Here the accelerations $\ddot{\boldsymbol{x}}$ are given by Eq.(D.5b). Futhermore, the stress resultant vector $\boldsymbol{\sigma}$, which can include damping effects, is represented by

$$
\sigma=S \varepsilon+D \dot{\varepsilon}+\sigma_{0}
$$

where $\boldsymbol{S}$ and $\boldsymbol{D}$ are the assembled stiffness and damping matrices, and $\boldsymbol{\sigma}_{0}$ is a vector of initial stress resultants.

Consider now small perturbations around some reference configuration specified by a set of degrees of freedom and its first and second time derivatives $\left(\boldsymbol{q}_{0}, \dot{\boldsymbol{q}}_{0}, \ddot{\boldsymbol{q}}_{0}\right)$ such that the actual variables are of the form

$$
\boldsymbol{q}=\boldsymbol{q}_{0}+\delta \boldsymbol{q}, \quad \dot{\boldsymbol{q}}=\dot{\boldsymbol{q}}_{0}+\delta \dot{\boldsymbol{q}}, \quad \ddot{\boldsymbol{q}}=\ddot{\boldsymbol{q}}_{0}+\delta \ddot{\boldsymbol{q}},
$$

where the prefix $\delta$ denotes a perturbation and should not be confused with the virtual variation operator. Expanding the equations of motion $\boldsymbol{g}$ in their Taylor series expansion and disregarding second and higher order terms yields,

$$
\boldsymbol{g}_{, \ddot{\boldsymbol{q}}} \delta \ddot{\boldsymbol{q}}+\boldsymbol{g}_{, \dot{\boldsymbol{q}}} \delta \dot{\boldsymbol{q}}+\boldsymbol{g}_{, \boldsymbol{q}} \delta \boldsymbol{q}=\mathbf{0}
$$


with

$$
\begin{aligned}
& \boldsymbol{g}_{, \boldsymbol{q}}=\boldsymbol{K}_{\boldsymbol{q}}+\boldsymbol{G}_{\boldsymbol{q}}+\boldsymbol{N}_{\boldsymbol{q}}, \\
& \boldsymbol{g}_{, \dot{q}}=\boldsymbol{D}_{\boldsymbol{q}}+\boldsymbol{C}_{\boldsymbol{q}}, \\
& \boldsymbol{g}_{, \ddot{q}}=\boldsymbol{M}_{\boldsymbol{q}}
\end{aligned}
$$

where $\boldsymbol{M}_{\boldsymbol{q}}$ is the mass matrix, $\boldsymbol{D}_{\boldsymbol{q}}$ is the damping matrix, $\boldsymbol{C}_{\boldsymbol{q}}$ is the velocity sensitivity matrix, $\boldsymbol{K}_{\boldsymbol{q}}$ is the structural stiffness matrix and $\boldsymbol{G}_{\boldsymbol{q}}$ and $\boldsymbol{N}_{\boldsymbol{q}}$ are the geometric stiffness and dynamic stiffness matrices, respectively. Expressions for these matrices are [38]:

$$
\begin{aligned}
\boldsymbol{M}_{\boldsymbol{q}}= & \mathcal{F}_{, \boldsymbol{q}}^{\mathrm{T}} \boldsymbol{M}_{\boldsymbol{x}} \mathcal{F}_{, \boldsymbol{q}}, \\
\boldsymbol{D}_{\boldsymbol{q}}= & \mathcal{E}_{, \boldsymbol{q}}^{\mathrm{T}} \boldsymbol{D} \mathcal{E}_{, \boldsymbol{q}} \\
\boldsymbol{C}_{\boldsymbol{q}}= & \mathcal{F}_{, \boldsymbol{q}}^{\mathrm{T}}\left(\left(\boldsymbol{M}_{\boldsymbol{x}} \ddot{\boldsymbol{x}}-\boldsymbol{f}\right)_{, \dot{\boldsymbol{x}}} \mathcal{F}_{, \boldsymbol{q}}+2 \boldsymbol{M}_{\boldsymbol{x}} \mathcal{F}_{, \boldsymbol{q}} \dot{\boldsymbol{q}}\right) \\
\boldsymbol{K}_{\boldsymbol{q}}= & \mathcal{E}_{, \boldsymbol{q}}^{\mathrm{T}} \boldsymbol{S} \mathcal{E}_{, \boldsymbol{q}} \\
\boldsymbol{G}_{\boldsymbol{q}}= & \mathcal{F}_{, \boldsymbol{q} \boldsymbol{q}}^{\mathrm{T}}\left(\boldsymbol{M}_{\boldsymbol{x}} \ddot{\boldsymbol{x}}-\boldsymbol{f}\right)+\mathcal{E}_{, \boldsymbol{q} \boldsymbol{q}}^{\mathrm{T}} \boldsymbol{\sigma} \\
\boldsymbol{N}_{\boldsymbol{q}}= & \mathcal{F}_{, \boldsymbol{q}}^{\mathrm{T}}\left(\left(\boldsymbol{M}_{\boldsymbol{x}} \ddot{\boldsymbol{x}}-\boldsymbol{f}\right)_{, \boldsymbol{x}} \mathcal{F}_{, \boldsymbol{q}}+\left(\boldsymbol{M}_{\boldsymbol{x}} \ddot{\boldsymbol{x}}-\boldsymbol{f}\right)_{, \dot{\boldsymbol{x}}} \mathcal{F}_{, \boldsymbol{q}} \dot{\boldsymbol{q}}+\right. \\
& \left.+\boldsymbol{M}_{\boldsymbol{x}}\left(\left(\mathcal{F}_{, \boldsymbol{q} \boldsymbol{q} \boldsymbol{q}} \dot{\boldsymbol{q}}\right) \dot{\boldsymbol{q}}+\mathcal{F}_{, \boldsymbol{q} q} \ddot{\boldsymbol{q}}\right)\right)+\mathcal{E}_{, \boldsymbol{q}}^{\mathrm{T}} \boldsymbol{D} \mathcal{E}_{, \boldsymbol{q} q} \dot{\boldsymbol{q}} .
\end{aligned}
$$

In the case the model is at rest and in equilibrium $(\dot{\boldsymbol{q}}=\mathbf{0}, \ddot{\boldsymbol{q}}=\mathbf{0})$, Eq.(E.5) simplifies to

$$
\begin{aligned}
\boldsymbol{M}_{\boldsymbol{q}} & =\mathcal{F}_{, \boldsymbol{q}}^{\mathrm{T}} \boldsymbol{M}_{\boldsymbol{x}} \mathcal{F}_{, \boldsymbol{q}}, \\
\boldsymbol{D}_{\boldsymbol{q}} & =\mathcal{E}_{, \boldsymbol{q}}^{\mathrm{T}} \boldsymbol{D} \mathcal{E}_{, \boldsymbol{q}}, \\
\boldsymbol{C}_{\boldsymbol{q}} & =\mathbf{0} \\
\boldsymbol{K}_{\boldsymbol{q}} & =\mathcal{E}_{, \boldsymbol{q}}^{\mathrm{T}} \boldsymbol{S E}_{, \boldsymbol{q}}, \\
\boldsymbol{G}_{\boldsymbol{q}} & =-\mathcal{F}_{, \boldsymbol{q} \boldsymbol{q}}^{\mathrm{T}} \boldsymbol{f}+\mathcal{E}_{, \boldsymbol{q} \boldsymbol{q}}^{\mathrm{T}} \boldsymbol{\sigma}, \\
\boldsymbol{N}_{\boldsymbol{q}} & =\mathbf{0} .
\end{aligned}
$$




\section{Reduced linearized equations of motion}

In this appendix the reduced linearized equations of motion are derived. The reduced mass and stiffness matrices derived here are used to compute the eigenfrequency results of the reduced models of Chapters 5, 6 and $\%$.

Substituting Eqs. (6.8) and (6.13a) in Eq. (6.7) and dropping the virtual variations $\delta \boldsymbol{z}$ yields the vector function $\boldsymbol{g}(\boldsymbol{z}, \dot{\boldsymbol{z}}, \ddot{\boldsymbol{z}})$

$$
\boldsymbol{g}=\left(\mathcal{F}_{, \boldsymbol{q}} \mathcal{G}_{, \boldsymbol{z}}\right)^{\mathrm{T}}\left(\boldsymbol{M}_{\boldsymbol{x}} \ddot{\boldsymbol{x}}-\boldsymbol{f}\right)+\left(\mathcal{E}_{, \boldsymbol{q}} \mathcal{G}_{, \boldsymbol{z}}\right)^{\mathrm{T}} \boldsymbol{\sigma}=\mathbf{0}
$$

which is an equivalent representation of the equations of motion (6.15). Here, by substituting Eqs.(6.13b) and (6.13c) in Eq.(6.6b), the accelerations $\ddot{\boldsymbol{x}}$ are given by

$$
\ddot{\boldsymbol{x}}=\mathcal{F}_{, q}\left(\mathcal{G}_{, z} \ddot{z}+\left(\mathcal{G}_{, z z} \dot{z}\right) \dot{z}\right)+\left(\mathcal{F}_{, q q} \mathcal{G}_{, z} \dot{z}\right) \mathcal{G}_{, z} \dot{z}
$$

Furthermore, the stress resultants $\boldsymbol{\sigma}$ are given by Eq.(E.2).

Consider now small perturbations around some reference configuration specified by a set of degrees of freedom and its first and second time derivatives $\left(\boldsymbol{z}_{0}, \dot{\boldsymbol{z}}_{0}, \ddot{\boldsymbol{z}}_{0}\right)$ such that the actual variables are of the form

$$
\boldsymbol{z}=\boldsymbol{z}_{0}+\delta \boldsymbol{z}, \quad \dot{\boldsymbol{z}}=\dot{\boldsymbol{z}}_{0}+\delta \dot{\boldsymbol{z}}, \quad \ddot{z}=\ddot{z}_{0}+\delta \ddot{z},
$$

where the prefix $\delta$ denotes a perturbation and should not be confused with the virtual variation operator. Expanding the equations of motion $\boldsymbol{g}$ in their Taylor series expansion and disregarding second and higher order terms yields,

$$
\boldsymbol{g}_{, \ddot{z}} \delta \ddot{\boldsymbol{z}}+\boldsymbol{g}_{, \dot{\boldsymbol{z}}} \delta \dot{\boldsymbol{z}}+\boldsymbol{g}_{, \boldsymbol{z}} \delta \boldsymbol{z}=\mathbf{0}
$$


with

$$
\begin{aligned}
& \boldsymbol{g}_{, \boldsymbol{z}}=\boldsymbol{K}_{\boldsymbol{z}}+\boldsymbol{G}_{\boldsymbol{z}}+\boldsymbol{N}_{\boldsymbol{z}}, \\
& \boldsymbol{g}_{, \dot{z}}=\boldsymbol{D}_{\boldsymbol{z}}+\boldsymbol{C}_{\boldsymbol{z}}, \\
& \boldsymbol{g}_{, \ddot{z}}=\boldsymbol{M}_{\boldsymbol{z}},
\end{aligned}
$$

where $\boldsymbol{M}_{\boldsymbol{z}}$ is the mass matrix, $\boldsymbol{D}_{\boldsymbol{z}}$ is the damping matrix, $\boldsymbol{C}_{\boldsymbol{z}}$ is the velocity sensitivity matrix, $\boldsymbol{K}_{\boldsymbol{z}}$ is the structural stiffness matrix and $\boldsymbol{G}_{\boldsymbol{z}}$ and $\boldsymbol{N}_{\boldsymbol{z}}$ are the geometric stiffness and dynamic stiffness matrices in terms of the reduced coordinates $\boldsymbol{z}$, respectively. Expressions for these matrices are

$$
\begin{aligned}
& M_{z}=\mathcal{G}_{, z}^{\mathrm{T}} \boldsymbol{M}_{q} \mathcal{G}_{, \boldsymbol{z}} \\
& \boldsymbol{D}_{\boldsymbol{z}}=\mathcal{G}_{, \boldsymbol{z}}^{\mathrm{T}} \boldsymbol{D}_{q} \mathcal{G}_{, \boldsymbol{z}}, \\
& \boldsymbol{C}_{\boldsymbol{z}}=\mathcal{G}_{, \boldsymbol{z}}^{\mathrm{T}} \boldsymbol{C}_{\boldsymbol{q}} \mathcal{G}_{, \boldsymbol{z}}+2\left(\mathcal{F}_{, \boldsymbol{q}} \mathcal{G}_{, \boldsymbol{z}}\right)^{\mathrm{T}} \boldsymbol{M}_{\boldsymbol{x}} \mathcal{F}_{, \boldsymbol{q}}\left(\mathcal{G}_{, \boldsymbol{z} z} \dot{\boldsymbol{z}}\right), \\
& \boldsymbol{K}_{\boldsymbol{z}}=\mathcal{G}_{, \boldsymbol{z}}^{\mathrm{T}} \boldsymbol{K}_{\boldsymbol{q}} \mathcal{G}_{, \boldsymbol{z}} \text {, } \\
& \boldsymbol{G}_{\boldsymbol{z}}=\mathcal{G}_{, \boldsymbol{z}}^{\mathrm{T}} \boldsymbol{G}_{\boldsymbol{q}} \mathcal{G}_{, \boldsymbol{z}}+\mathcal{G}_{, \boldsymbol{z} \boldsymbol{z}}^{\mathrm{T}}\left(\mathcal{F}_{, \boldsymbol{q}}^{\mathrm{T}}\left(\boldsymbol{M}_{\boldsymbol{x}} \ddot{\boldsymbol{x}}-\boldsymbol{f}\right)+\mathcal{E}_{, \boldsymbol{q}}^{\mathrm{T}} \boldsymbol{\sigma}\right), \\
& \boldsymbol{N}_{\boldsymbol{z}}=\mathcal{G}_{, \boldsymbol{z}}^{\mathrm{T}} \boldsymbol{N}_{\boldsymbol{q}} \mathcal{G}_{, \boldsymbol{z}}+\left(\mathcal{F}_{, q} \mathcal{G}_{, \boldsymbol{z}}\right)^{\mathrm{T}}\left(\left(\boldsymbol{M}_{\boldsymbol{x}} \ddot{\boldsymbol{x}}-\boldsymbol{f}\right)_{, \dot{\boldsymbol{x}}} \mathcal{F}_{, q} \mathcal{G}_{, \boldsymbol{z} z} \dot{\boldsymbol{z}}+\right. \\
& \left.+\boldsymbol{M}_{\boldsymbol{x}}\left(2\left(\mathcal{F}_{, q q} \dot{\boldsymbol{q}}\right)\left(\mathcal{G}_{, z z} \dot{z}\right)+\mathcal{F}_{, q}\left(\mathcal{G}_{, z z} \ddot{z}+\left(\mathcal{G}_{, z z z} \dot{z}\right) \dot{z}\right)\right)\right)+ \\
& +\left(\mathcal{E}_{, \boldsymbol{q}} \mathcal{G}_{, \boldsymbol{z}}\right)^{\mathrm{T}} \boldsymbol{D} \mathcal{E}_{, \boldsymbol{q}} \mathcal{G}, \boldsymbol{z} \boldsymbol{z} \dot{\boldsymbol{z}},
\end{aligned}
$$

where $\boldsymbol{M}_{\boldsymbol{q}}, \boldsymbol{D}_{\boldsymbol{q}}, \boldsymbol{C}_{\boldsymbol{q}}, \boldsymbol{K}_{\boldsymbol{q}}, \boldsymbol{G}_{\boldsymbol{q}}$ and $\boldsymbol{N}_{\boldsymbol{q}}$ are the matrices from Eq.(E.5). For the FBM of Chapter 6 the matrices $\mathcal{G}_{, \boldsymbol{z} z}$ and $\mathcal{G}_{, \boldsymbol{z z} \boldsymbol{z}}$ are zero whereas for the IBM of Chapter 6, the matrix $\mathcal{G}_{, \boldsymbol{z z} z}$ can be constructed with

$$
\begin{aligned}
\mathcal{G}_{, s s s} & =\mathcal{Q}_{, s s s}+\mathcal{V}_{, s s s} \boldsymbol{\eta}, \\
\mathcal{G}_{,{ }_{\text {nss }}} & =\mathcal{G}_{, s \boldsymbol{\eta} s}=\mathcal{G}_{, s s \boldsymbol{\eta}}=\mathcal{V}_{, s s}(s),
\end{aligned}
$$

where all other terms are zero. Here, the terms $\mathcal{Q}$,sss and $\mathcal{V}_{\text {,sss }}$ are constant for cubic interpolation of the gross reference motion and the modal subspace, respectively.

In the case the reduced model is at rest and in equilibrium, Eq.(F.5) reduces to

$$
\begin{aligned}
\boldsymbol{M}_{\boldsymbol{z}} & =\mathcal{G}_{, \boldsymbol{z}}^{\mathrm{T}} \boldsymbol{M}_{\boldsymbol{q}} \mathcal{G}_{, \boldsymbol{z}}, \\
\boldsymbol{D}_{\boldsymbol{z}} & =\mathcal{G}_{, \boldsymbol{z}}^{\mathrm{T}} \boldsymbol{D}_{\boldsymbol{q}} \mathcal{G}_{, \boldsymbol{z}}, \\
\boldsymbol{C}_{\boldsymbol{z}} & =\mathbf{0} \\
\boldsymbol{K}_{\boldsymbol{z}} & =\mathcal{G}_{, \boldsymbol{z}}^{\mathrm{T}} \boldsymbol{K}_{\boldsymbol{q}} \mathcal{G}_{, \boldsymbol{z}}, \\
\boldsymbol{G}_{\boldsymbol{z}} & =\mathcal{G}_{, \boldsymbol{z}}^{\mathrm{T}} \boldsymbol{G}_{q} \mathcal{G}_{, \boldsymbol{z}}+\mathcal{G}_{, \boldsymbol{z} \boldsymbol{z}}^{\mathrm{T}}\left(-\mathcal{F}_{, \boldsymbol{q}}^{\mathrm{T}} \boldsymbol{f}+\mathcal{E}_{, \boldsymbol{q}}^{\mathrm{T}} \boldsymbol{\sigma}\right), \\
\boldsymbol{N}_{\boldsymbol{z}} & =\mathbf{0}
\end{aligned}
$$




\section{Dankwoord}

Zonder de steun, invloeden en bijdragen van heel wat mensen zou dit proefschrift er totaal anders uit gezien hebben of misschien wel nooit werkelijkheid geworden zijn. Deze mensen verdienen natuurlijk een bedankje!

Allereerst wil ik mijn directe begeleiders, Ben Jonker, Dannis Brouwer en Ronald Aarts, bedanken. Ben, bedankt voor je onuitputtelijke enthousiasme voor mijn onderzoek. Ondanks dat je al met pensioen bent, ben je me blijven begeleiden en daar ben ik erg dankbaar voor. We hebben heel wat interessante discussies gevoerd over multibody dynamica en ik kijk daarom ook uit naar de eerste druk van je boek over dit onderwerp! Dannis, bedankt voor alle discussies die we hebben gevoerd over compliant mechanisms en je enthousiasme voor de 'two-dof' testopstelling die nu zelfs terug te vinden is in je les materiaal over precision mechanisms! Daarnaast heb ik je humor altijd erg goed kunnen waarderen. Ons uitje naar Como was toch wel heel hilarisch! Ronald, bedankt dat ik altijd bij je terecht kon als ik weer eens vast zat met m'n onderzoek. Meestal na een goede discussie met jou kon ik weer verder en meerdere malen was er zelfs sprake van een ware doorbraak. Ik heb erg genoten van onze samenwerking.

De overige leden van mijn commissie, André de Boer, Marcel Ellenbroek, Herman van der Kooij en Olivier Brüls, zou ik ook graag willen bedanken voor het lezen en beoordelen van mijn proefschrift. In het bijzonder, Marcel bedankt voor de interesse die je de afgelopen jaren toonde in mijn onderzoek en voor alle stevige discussies die we erover hebben gevoerd.

Daarnaast wil ik ook graag Herman Soemers, Jaap Meijaard, Leo Tiemersma, Wouter Hakvoort, Volkert van der Wijk en Martina Tjapkes bedanken. Herman, bedankt voor je betrokkenheid aan het begin van het project. Ik vond het jammer dat we door externe factoren onze samenwerking moesten staken. Jaap, bedankt voor het doornemen van mijn artikelen en voor het vinden van al die kleine foutjes! Leo, bedankt voor al je werk in het lab om de 'two-dof' testopstelling te realiseren. Wouter bedankt voor onze samenwerking op het gebied van systeem model reductie. Volkert bedankt voor onze interessante discussies over kinematica, dynamica, balanceren en model reductie. En Martina bedankt voor al de organisatorische hulp met betrekking tot mijn promotie en dat je er ook voor me was als ik even moest ontladen! 
Al mijn andere collega's van de vakgroep Werktuigbouwkundige Automatisering bedank ik voor de gezelligheid tijdens de vakgroep uitjes, promotie feestjes en alle koffiepauzes. Specially Johann, Jozef, Justus and Paul. Thanks for all the breaks at your office and our beer drinking evenings (Johann, I still blame you that I didn't finish on time :p)!

Tevens bedank ik mijn voormalige studenten, Rick Hoitzing, Ger Folkersma, Derk ten Hoopen, Hedzer Wiersma en Willem Wijma voor het werk wat zij tijdens hun afstuderen voor mij hebben gedaan. Rick, Ger en Willem, bedankt voor al het conceptuele werk voor, de realisatie van, en voor al het meetwerk aan de 'two-dof' opstelling. Rick en Willem, ik hoop dat jullie carrière bij VDL voorspoedig zal verlopen. Ger, nog veel succes met je eigen promotieonderzoek de komende jaren. Derk, bedankt voor je verkennende werk over systeem model reductie. Dit vormde toch de basis voor deel 2 van mijn proefschrift. En Hedzer bedankt voor het uitmuntende werk wat je hebt geleverd over het ontwerpen van optimale grote slag elastische scharnieren (+patent!). Ook jullie carrière wens ik het beste toe!

Af en toe is het ook eens goed om tijd te maken voor ontspannende bezigheden die niets te maken hebben met promoveren. Graag bedank ik Alexander, Bob, Rick, Toon, Jeroen, Sietse en Niels voor de lol tijdens de Batavierenraces en avonden uit. Sietse en Niels ook bedankt voor de gezellige basketbal jaren bij Arriba, waar ik eveneens ook mijn andere teamgenoten en in het speciaal Anne, Hans en Remert voor wil bedanken!

Furthermore, I would like to thank Alexandre Paternoster for letting me use his LaTeX template files for my thesis. This saved me a lot of work! Also, a special thanks to Hélène, for being there and supporting me this last year. Though our paths now go separate ways, I wish you all the best nonetheless. My last thanks in English goes to Johann. Thanks for being there during all the ups and downs, for all the evenings drinking beer, for all the crazy fun we had and for agreeing to be one of my paranymphs. I know it is probably a mistake to give you more compliments, but you are awesome. Thanks for being my friend these years!

Als laatste wil ik mijn ouders, broer, broertje, zus en zwager bedanken voor hun liefde en steun gedurende dit promotietraject. Ik had het niet zonder jullie gekund. In het bijzonder, Ro bedankt voor het ontwerpen van de omslag en Gijs bedankt voor alle hulp en uitleg die je me hebt gegeven over programmeren waardoor ik al mijn model reductie ideeën kon realiseren!

Met het einde van mijn promotieonderzoek, komt er voor mij ook een einde aan het hoofdstuk Enschede. Al weer tien jaar geleden ben ik in Enschede begonnen met mijn werktuigbouw studie en al die tijd heb ik er met erg veel plezier gewoond, gestudeerd en gewerkt. Nu wordt het echter tijd voor iets nieuws en ik ben dan ook erg benieuwd naar het volgende hoofdstuk in m'n leven! 


\section{List of publications}

\section{Patent}

- Brouwer, D.M., Wiersma, D.H., Boer, S.E., Aarts, R.G.K.M.: Flexure joint for high precision relative movement of devices or parts of devices, University Of Twente, Patent No. 12194573.7, November, 2012.

\section{Journal articles}

First author:

- Boer, S.E., ten Hoopen, D., Aarts, R.G.K.M., Hakvoort, W.B.J., Jonker, J.B.: Model reduction for efficient time-integration of planar flexible multibody models, International Journal of Non-Linear Mechanics, 53, 75-82 (2013).

- Boer, S.E., Aarts, R.G.K.M., Hakvoort. W.B.J.: Model Reduction for efficient time-integration of spatial flexible multibody models, Multibody System Dynamics, in press (2013), doi: 10.1007/s11044-013-9346-y.

- Boer, S.E., Aarts, R.G.K.M., Meijaard, J.P., Brouwer, D.M., Jonker, J.B.: A nonlinear two-node superelement for use in flexible multibody systems, Multibody System Dynamics, in press (2013), doi: 10.1007/s11044-013-9373-8.

- Boer, S.E., Aarts, R.G.K.M., Meijaard, J.P., Brouwer, D.M., Jonker, J.B.: A nonlinear two-node superelement with deformable-interface surfaces for use in flexible multibody systems, Multibody System Dynamics, under review (2013).

Co-author:

- Brouwer, D.M., Boer, S.E., Meijaard, J.P., Aarts, R.G.K.M.: Optimization of release locations for small stress large stiffness flexure mechanisms, Mechanism and Machine Theory, 64, 230-250 (2013). 
- Brouwer, D.M., Folkersma, K.G.P., Boer, S.E., Aarts, R.G.K.M.: Exact constraint design of a two-degree of freedom flexure-based machanism, Journal of mechanisms and robotics, in press (2013).

- Wiersma, D.H., Boer, S.E., Brouwer, D.M., Aarts, R.G.K.M.: Design and optimization of large rotation high stiffness flexures, ASME Journal of Computational and Nonlinear Dynamics (CND), under review (2013).

\section{Conference articles}

First author:

- Boer, S.E., Aarts, R.G.K.M., Brouwer, D.M., Jonker, J.B.: Multibody modelling and optimization of a curved hinge flexure, In: The $1^{\text {st }}$ Joint International Conference on Multibody System Dynamics, Lappeenranta, Finland (2010).

- Boer, S.E., Aarts, R.G.K.M., Meijaard, J.P., Brouwer, D.M., Jonker, J.B.: A two-node superelement description for modelling of flexible complex-shaped beam-like components, In: ECCOMAS Thematic Conference Multibody Dynamics 2011, Brussels, Belgium (2011).

- Boer, S.E., ten Hoopen, D., Aarts, R.G.K.M., Hakvoort, W.B.J., Jonker, J.B.: Model reduction for efficient time-integration of planar non-linear flexible multibody models, In: The $2^{\text {nd }}$ Joint International Conference on Multibody System Dynamics, Stuttgart, Germany (2012).

Co-author:

- Aarts, R.G.K.M., Boer, S.E., Meijaard, J.P., Brouwer, D.M., Jonker, J.B.: Analyzing overconstrained design of compliant mechanisms, In: Proceedings of the ASME 2011 International Design Engineering Technical Conferences \& Computers and Information in Engineering Conferences (IDETC/CIE 2012), Washington, DC, USA (2011).

- Brouwer, D.M., Boer, S.E., Aarts, R.G.K.M., Meijaard, J.P., Jonker, J.B.: Freedom and constraint analysis and optimization, In: Proceedings of the 26th annual meeting of the American society of precision engineering, Denver, USA (2011).

- Folkersma, K.G.P., Boer, S.E., Brouwer, D.M., Herder, J.L., Soemers, H.M.J.R.: A 2-DOF large stroke flexure based positioning mechanism, In: Proceedings of the ASME 2012 International Design Engineering Technical Conferences \& Computers and Information in Engineering Conferences (IDETC/CIE 2012), Chicago, IL, USA (2012). 
- Wiersma, D.H., Boer, S.E., Aarts, R.G.K.M., Brouwer, D.M.: Large stroke performance optimization of spatial flexure hinges, In: Proceedings of the ASME 2012 International Design Engineering Technical Conferences \& Computers and Information in Engineering Conferences (IDETC/CIE 2012), Chicago, IL, USA (2012).

- Wijma, W., Boer, S.E., Aarts, R.G.K.M., Brouwer, D.M., Hakvoort, W.B.J.: Modal measurements and model corrections of a large stroke compliant mechanism, In: ECCOMAS Thematic Conference Multibody Dynamics 2013, Zagreb, Croatia (2013). 



\section{References}

[1] Aarts, R.G.K.M., Jonker J.B.: Dynamic simulation of planar flexible link manipulators using adaptive modal integration, Multibody System Dynamics, 7(1), 31-50 (2002).

[2] Aarts, R.G.K.M., van Dijk, J., Brouwer, D.M., Jonker, J.B.: Application of flexible multibody modelling for control synthesis in mechatronics, In: ECCOMAS Thematic Conference Multibody Dynamics 2011, Brussels, Belgium (2011).

[3] Aarts, R.G.K.M., Meijaard, J.P., Jonker, J.B.: Flexible multibody modelling for exact constraint design of compliant mechanisms, Multibody System Dynamics, 27(1), 119-133 (2012).

[4] Agrawal, O.P., Shabana, A.A.: Dynamic analysis of multibody systems using component modes, Computers and Structures, 21(6), 1303-1312 (1985).

[5] Amsallem, D., Cortial, J., Carlberg, K., Farhat, C.: A method for interpolating on manifolds structural dynamics reduced-order models, Internatial Journal for Numerical Methods in Engineering, 80(9), 1241-1258 (2009).

[6] Arnoldi, W.E.: The principle of minimized iterations in the solution of the matrix eigenvalue problem, Quarterly of Applied Mathematics, 9(17), 17-29 (1951).

[7] Awtar, S., Shimotsu, K., Sen, S.: Elastic averaging in flexure mechanisms: A Three-Beam Parallelogram Flexure Case Study, Journal of Mechanisms and Robotics, 2(4), 041006 (2010).

[8] Bauchau, O.A., Rodriguez, J.: Formulation of modal-based elements in nonlinear flexible multibody dynamics, International Journal for Multiscale Computational Engineering, 1(2\&3), 161-180 (2003).

[9] Besseling, J.F.: Non-linear theory for elastic beams and rods and its finite element representation, Computer Methods in Applied Mechanics and Engineering, 31(2), 205-220 (1982). 
[10] Blanding, D.: Exact constraint machine design using kinematic principles, ASME Press, New York (1999).

[11] Boer, S.E., Aarts, R.G.K.M., Meijaard, J.P., Brouwer, D.M., Jonker, J.B.: A two-node superelement description for modelling of flexible complex-shaped beam-like components, In: ECCOMAS Thematic Conference Multibody Dynamics 2011, Brussels, Belgium (2011).

[12] Boer, S.E., Aarts, R.G.K.M., Meijaard, J.P., Brouwer, D.M., Jonker, J.B.: A nonlinear two-node superelement for use in flexible multibody systems, Multibody System Dynamics, in press (2013), doi: 10.1007/s11044-013-9373-8.

[13] Boer, S.E., Aarts, R.G.K.M., Hakvoort. W.B.J.: Model reduction for efficient time-integration of spatial flexible multibody models, Multibody System Dynamics, in press (2013), doi: 10.1007/s11044-013-9346-y.

[14] Boer, S.E., ten Hoopen, D., Aarts, R.G.K.M., Hakvoort, W.B.J., Jonker, J.B.: Model reduction for efficient time-integration of planar flexible multibody models, International Journal of Non-Linear Mechanics, 53, 75-82 (2013).

[15] Bois, J.L.d., Adhikari, S., Lieven, N.A.J.: On the quantification of eigenvalue curve veering: A Veering Index, Journal of Applied Mechanics, 78(4) (2011).

[16] Brouwer, D.M., Boer, S.E., Meijaard, J.P., Aarts, R.G.K.M.: Optimization of release locations for small stress large stiffness flexure mechanisms, Mechanism and Machine Theory, 64, 230-250 (2013).

[17] Brouwer, D.M., Folkersma, K.G.P., Boer, S.E., Aarts, R.G.K.M.: Exact constraint design of a two-degree of freedom flexure-based machanism, Journal of mechanisms and robotics, in press (2013).

[18] Brüls, O., Duysinx, P., Golinval, J.C.: The global modal parameterization for non-linear model-order reduction in flexible multibody dynamics, Internatial Journal for Numerical Methods in Engineering, 69(5), 948-977 (2007).

[19] Cardona, A., Géradin, M.: Modelling of superelements in mechanism analysis, Internatial Journal for Numerical Methods in Engineering, 32(8), 1565-1593 (1991).

[20] Cardona, A., Géradin, M.: A superelement formulation for mechanism analysis, Computer Methods in Applied Mechanics and Engineering, 100(1), 1-29 (1992).

[21] Cardona, A.: Superelements Modelling in Flexible Multibody Dynamics, Multibody System Dynamics, 4(2-3), 245-266 (2000).

[22] Chen, H.H., Lin, W.Y., Hsiao, K.M.: Co-rotational finite element formulation for thin-walled beams with generic open section, Computer Methods in Applied Mechanics and Engineering, 195(19-22), 2334-2370 (2006). 
[23] Craig, Jr. R.R., Bampton, M.C.C.: Coupling of substructures for dynamic analyses, American Institute of Aeronautics and Astronautics Journal, 6(7), 1313-1319 (1968).

[24] Craig, Jr. R.R.: Substructure Methods in Vibration, Journal of Vibration and Acoustics, 117, 207-213 (1995).

[25] Dormand, J.R., Prince, P.J.: A family of embedded Runge-Kutta formulae, Journal of Computational and Applied Mathematics, 6(1), 19-26 (1980).

[26] Ewins, D.J.: Modal testing: theory, practice and application, Research Studies Press, (2000).

[27] Folkersma, K.G.P., Boer, S.E., Brouwer, D.M., Herder, J.L., Soemers, H.M.J.R.: A 2-DOF large stroke flexure based positioning mechanism, In: Proceedings of the ASME 2012 International Design Engineering Technical Conferences \& Computers and Information in Engineering Conferences (IDETC/CIE 2012), Chicago, IL, USA (2012).

[28] Géradin, M., Cardona, A.: Flexible multibody dynamics: A finite element approach., John Wiley and Sons, Chichester (2001).

[29] Gonçalves, R., Ritto-Corrêa, M., Camotim, D.: A large displacement and finite rotation thin-walled beam formulation including cross-section deformation, Computer Methods in Applied Mechanics and Engineering, 199(23-24), 16271643 (2010).

[30] Gruttmann, F., Sauer, R., Wagner, W.: Theory and numerics of threedimensional beams with elastoplastic material behaviour, Internatial Journal for Numerical Methods in Engineering, 48(12), 1675-1702 (2000).

[31] Guyan, R.J.: Reduction of stiffness and mass matrices, American Institute of Aeronautics and Astronautics Journal, 3(2), 380 (1965).

[32] Hale, L.C.: Principles and techniques for designing precision machines, PhD Thesis, M.I.T., Cambridge Massachusetts (1999).

[33] Heirman, G. H. K., Naets, F., Desmet, W.: A system-level model reduction technique for the efficient simulation of flexible multibody systems, Internatial Journal for Numerical Methods in Engineering, 85(3), 330-354 (2011).

[34] Hoitzing, F.: Kinematic and dynamic modeling for the conceptual design of a 2-DOFs large stroke elastic positioning mechanism, Master Thesis, University of Twente, Enschede (2010).

[35] Hsiao, K.M., Lin, W.Y.: A co-rotational formulation for thin-walled beams with monosymmetric open section, Computer Methods in Applied Mechanics and Engineering, 190(8-10), 1163-1185 (2000). 
[36] Hurty, W.C.: Vibrations of structural systems by component mode synthesis, Journal of Engineering Mechanics/American Society of Civil Engineers, 86(4), 51-69 (1960).

[37] Hurty, W.C.: Dynamic analysis of structural systems using component modes, American Institute of Aeronautics and Astronautics Journal, 3(4), 678-685 (1965).

[38] Jonker, J.B.: A finite element dynamic analysis of spatial mechanisms with flexible links, Computer Methods in Applied Mechanics and Engineering, 76(1), 17-40 (1989).

[39] Jonker, J.B., Meijaard, J.P.: SPACAR - Computer program for dynamic analysis of flexible spatial mechanisms and manipulators, In: Schiehlen, W. (ed.) Multibody Systems Handbook. pp. 123-143. Springer-Verlag, Berlin (1990).

[40] Jonker, J.B.: Linearization of dynamic equations of flexible mechanisms-a finite element approach, Internatial Journal for Numerical Methods in Engineering, 31(7), 1375-1392 (1991).

[41] Jonker, J.B., Meijaard, J.P.: Definition of deformation parameters for the beam element and their use in flexible multibody system analysis, In: ECCOMAS Thematic Conference Multibody Dynamics 2009, Warsaw University of Technology, Warsaw (2009).

[42] Jonker, J.B., Aarts, R.G.K.M., van Dijk, J.: A linearized input-output representation of flexible multibody systems for control synthesis, Multibody System Dynamics, 21(2), 99-122 (2009).

[43] Jonker, J.B., Meijaard, J.P.: A geometrically non-linear formulation of a three-dimensional beam element for solving large deflection multibody system problems, International Journal of Non-Linear Mechanics, 53, 63-74 (2013).

[44] Kerschen, G., Golinval, J.C., Vakakis, A., Bergman, L.: The method of proper orthogonal decomposition for dynamical characterization and order reduction of mechanical systems: an overview, Nonlinear Dynamics, 41(1-3), 147-169 (2005).

[45] Kim, S.S., Haug, E.J.: A recursive formulation for flexible multibody dynamics, part I: Open-loop systems, Computer Methods in Applied Mechanics and Engineering, 71(3), 293-314 (1988).

[46] De Klerk, D., Rixen, D.J., Voormeeren, S.N.: General framework for dynamic substructuring: History, review, and classification of techniques, American Institute of Aeronautics and Astronautics Journal, 46(5), 1169-1181 (2008).

[47] Lanczos, C.: An iteration method for the solution of the eigenvalue problem of linear differential and integral operators, Journal of Research of the National Bureau of Standards, 45(4), 255-282 (1950). 
[48] Lehner, M., Eberhard, P.: On the use of moment-matching to build reduced order models in flexible multibody dynamics, Multibody System Dynamics, 16(2), 191211 (2006).

[49] Lehner, M., Eberhard, P.: A two-step approach for model reduction in flexible multibody dynamics, Multibody System Dynamics, 17(2-3), 157-176 (2007).

[50] Meijaard, J.P.: Validation of flexible beam elements in dynamics programs, Nonlinear Dynamics, 9(1-2), 21-36 (1996).

[51] Meijaard, J.P., Brouwer, D.M., Jonker, J.B.: Analytical and experimental investigation of a parallel leaf spring guidance, Multibody System Dynamics, 23(1), 77-97 (2010).

[52] Moore, B.: Principal component analysis in linear systems: Controllability, observability, and model reduction, IEEE Transactions on Automatic Control, 26(1), 17-32 (1981).

[53] Moore, G.E.: Cramming more components onto integrated circuits, Electronics, 38(8), 114-117 (1965).

[54] Naets, F., Heirman, G.H.K., Vandepitte, D., Desmet, W.: Inertial force term approximations for the use of Global Modal Parameterization for planar mechanisms, Internatial Journal for Numerical Methods in Engineering, 85(4), 518-536 (2011).

[55] Naets, F., Tamarozzi, T., Heirman, G.H.K., Desmet, W.: Real-time flexible multibody simulation with Global Modal Parameterization, Multibody System Dynamics, 27(3), 267-284 (2012).

[56] Perkins, N.C., Mote Jr, C.D.: Comments on curve veering in eigenvalue problems, Journal of Sound and Vibration, 106(3), 451-463 (1986).

[57] Schiehlen, W., Leister, G.: Benchmark-beispiele des DFG-schwerpunktprogrammes dynamik von mehrkörpersystemen, Zwishenbericht ZB-64, Universität Stuttgart, Institut B für Mechanik (1991).

[58] Seshu, P.: Substructuring and component mode synthesis, Shock and Vibration, 4(3), 199-210 (1997).

[59] Shabana, A.A., Wehage, R.A.: A coordinate reduction technique for dynamic analysis of spatial substructures with large angular rotations, Journal of Structural Mechanics, 11(3), 401-431 (1983).

[60] Shabana, A.A.: Substructure synthesis methods for dynamic analysis of multibody systems, Computers and Structures, 20(4), 737-744 (1985).

[61] Shabana, A.A., Schwertassek, R.: Equivalence of the floating frame of reference approach and finite element formulations, International Journal of Non-Linear Mechanics, 33(3), 417-432 (1998). 
[62] Shabana, A.A.: Dynamics of multibody systems, Cambridge University Press, Cambridge (2005).

[63] Shampine, L.F., Gordon, M.K.: Computer solution of ordinary differential equations: The Initial Value Problem, Freeman, San Francisco (1975).

[64] Simo, J.C., Vu-Quoc, L.: A Geometrically-exact rod model incorporating shear and torsion-warping deformation, International Journal of Solids and Structures, 27(3), 371-393 (1991).

[65] Soemers, H.M.J.R.: Design principles for precision mechanisms, T-Pointprint, Enschede (2010)

[66] Timoshenko, S.P., Goodier, J.N.: Theory of elasticity., McGraw-Hill Book Company, New York (1951).

[67] Visser, W., Besseling, J.F.: Large displacement analysis of beams, Report WTHD-10, Laboratory for Engineering Mechanics, Delft University of Technology (1969).

[68] Wiersma, D.H., Boer, S.E., Aarts, R.G.K.M., Brouwer, D.M.: Large stroke performance optimization of spatial flexure hinges, In: Proceedings of the ASME 2012 International Design Engineering Technical Conferences \& Computers and Information in Engineering Conferences (IDETC/CIE 2012), Chicago, IL, USA (2012).

[69] Wiersma, D.H., Boer, S.E., Brouwer, D.M., Aarts, R.G.K.M.: Design and optimization of large rotation high stiffness flexures, ASME Journal of Computational and Nonlinear Dynamics (CND), submitted (2012).

[70] Wijma, W., Boer, S.E., Aarts, R.G.K.M., Brouwer, D.M., Hakvoort, W.B.J.: Modal measurements and model corrections of a large stroke compliant mechanism, In: ECCOMAS Thematic Conference Multibody Dynamics 2013, Zagreb, Croatia (2013).

[71] Wu, S.C., Haug, E.J.: Geometric non-linear substructuring for dynamics of flexible mechanical systems, Internatial Journal for Numerical Methods in Engineering, 26(10), 2211-2226 (1988).

[72] Yoo, W.S., Haug, E.J.: Dynamics of articulated structures. Part I. Theory, Journal of Structural Mechanics, 14(1), 105-126 (1986).

[73] Yoo, W.S., Haug, E.J.: Dynamics of articulated structures. Part II. Computer implementation and applications, Journal of Structural Mechanics, 14(2), 177 189 (1986). 


\section{Model Reduction of Flexible Multibody Systems}

Numerical simulations are essential to determine the characteristics, performance and structural integrity of mechanisms and robots. With increasingly higher demands on the specifications of such devices, the demands on the accuracy of the numerical models increases as well. Increasing the complexity of the models, inherently increases the computational time of the simulations. Model reduction techniques can offer a reduction of the simulation time, while maintaining sufficient accuracy. In this work, the modelling of flexible multibody systems is considered. The starting point is a numerical approach based on non-linear finite elements such as beams, trusses and hinges. In particular the spatial beam element has proven to offer a numerical efficient analysis of mechanisms that consist of beamlike components. However, for systems with complex-shaped parts, or for systems composed of a rather large number of beams, the dimensionality of the model increases. Hence, two types of model reduction techniques are investigated for the efficient and accurate modelling of such flexible multibody systems. The first type deals with the efficient and accurate modelling of individual components that may be flexible and have a complex geometry. This is referred to as component model reduction. The second type attempts to reduce a complete non-linear multibody system and can therefore be referred to as a system model reduction approach. Both methods are demonstrated by modelling a large-stroke two degree of freedom compliant positioning mechanism. 